\author{
Universidade de São Paulo \\ Instituto de Física
}

\title{
Investigação cinética de modos geodésicos de baixas frequências em plasmas magnetizados
}

\author{
Reneé Jordashe Franco Sgalla
}

Orientador: Prof. Dr. Artour G. Elfimov

Co-Orientador: Prof. Dr. Ricardo Magnus Osório Galvão

Tese de doutorado apresentada ao Instituto de Física para a obtenção do título de Doutor em Ciências

Banca Examinadora:

Prof. Dr. Artour G. Elfimov (IF - USP )

Prof. Dr. Ivan Cunha Nascimento (IF - USP)

Prof. Dr. Ricardo Luiz Viana (UFPR)

Prof. Dr. Luiz Fernando Ziebell (UFRGS)

Prof. Dr. Francisco Eugenio Mendonça da Silveira (UFABC) 


\section{FICHA CATALOGRÁFICA \\ Preparada pelo Serviço de Biblioteca e Informação do Instituto de Física da Universidade de São Paulo}

Sgalla, Reneé Jordashe Franco

Investigação cinética de modos geodésicos de baixas frequências em plasmas magnetizados. São Paulo, 2014.

Tese (Doutorado) - Universidade de São Paulo. Instituto de Física. Depto. de Física Aplicada.

Orientador: Prof. Dr. Artour G. Elfimov

Área de Concentração: Física

Unitermos: 1. Física de plasmas; 2. Fusão nuclear; 3. Teoria cinética; 4. Magnetohidrodinâmica; 5 . Turbulência eletrostática.

USP/IF/SBI-057/2014 


\section{Dedicatória}

Dedico esta tese à minha querida esposa, Mila Silva Costa, que sempre me apoiou em minhas atividades acadêmicas, à minha filha, Helena Costa Sgalla, a quem espero conseguir deixar uma herança de conhecimentos para toda a vida e aos meus pais, Remo Sgalla e Maria Alice Franco Sgalla, a quem devo todas as minhas conquistas pessoais e profissionais. 


\section{Agradecimentos}

A realização deste trabalho não foi fruto somente do meu esforço e da minha dedicação, mas também da colaboração de pessoas e instituições às quais devo sinceros agradecimentos.

Agradeço ao Conselho Nacional de Desenvolvimento Científico e Tecnológico (CNPq) pelo apoio financeiro, por meio da bolsa "Doutorado Pleno (GD)", que me foi concedido durante o presente programa de doutoramento. O referido apoio me proporcionou, além de condições financeiras essenciais, motivação suficiente para poder levar este trabalho adiante e, em consequência deste, os próximos que provavelmente se seguirão.

Em especial, a minha querida esposa, Mila Silva Costa, devo meu mais sincero reconhecimento pelo apoio emocional que, desde o final do meu mestrado, tem sido de inestimável ajuda para seguir adiante em minha carreira acadêmica. Também reconheço a ajuda prestada por ela quanto à revisão gramatical e estilística da escrita desta tese.

Agradeço também a minha filha, Helena Costa Sgalla, pela motivação para continuar adiante, principalmente nos momentos mais difíceis, que não foram poucos.

Não poderia esquecer de agradecer também a minha mãe, Maria Alice Franco Sgalla, pelos cuidados, ajuda e carinho que ela teve com a Helena e que me permitiram dedicar uma boa parte do meu tempo para o meu doutorado. Da mesma forma agradeço ao meu falecido pai, Remo Sgalla, pela minha educação e formação, que certamente teve influencia no meu doutorado, e pela sua ajuda durante minhas viagens com intuitos acadêmicos.

Ao meu orientador, Prof. Dr. Artour Grigorievich Elfimov, que revisou meus cálculos e sempre esteve presente para discussões sobre a física de tokamaks, devo boa parte da realização deste trabalho, cujas contribuições com relação às diversas correções desta tese por ele fornecidas foram essenciais para atingir a forma atual.

Uma grande parte do conhecimento que adquiri com relação à aplicação do modelo girocinético e da teoria de dois fluidos ao estudo de modos geodésicos em tokamaks devo ao Prof. Dr. Andrei I. Smolyakov, com quem tive o prazer de trabalhar em uma colaboração que resultou na maior parte dos capítulos desta tese e em trabalhos publicados e apresentados em conferências. Além disso, boa parte das referências bibliográficas desta tese foram de sua recomendação.

Ao Prof. Dr. Ricardo Magnus Osório Galvão agradeço pelo curso de Física de Plasmas I ministrado no $2^{\circ}$ semestre de 2011, o qual me ajudou melhorar meus conhecimentos em física de plasma de uma maneira geral. Também agradeço a ele as discussões sobre rotação de plasma, as quais contaram também com a participação do Prof. Dr. Artour G. Elfimov.

Ao meu colega Davi Ciro Taborda, além dos excelentes cafés sem açúcar após almoço em sua sala, agradeço pelas frutíferas discussões sobre equilíbrio MHD e outros assuntos importantes sobre conhecimentos de fronteira em física de plasma. Agradeço também ao Davi a ajuda prestada na edição de algumas das figuras desta tese. 


\section{Resumo}

Devido à sua importância em turbulência causada por ondas de deriva e à aplicação com propósitos em diagnósticos de plasma, a investigação de fluxos zonais (ZF) e modos acústicos geodésicos (GAM) tem atraído bastante atenção na literatura em física de plasmas. Nesta tese, primeiramente consideramos efeitos de equilíbrio com rotação poloidal e toroidal nestes modos, posteriormente investigamos efeitos diamagnéticos em GAM a partir de um modelo de dois fluido, no qual incluímos viscosidade paralela de íons e, na parte final, consideramos amortecimento de Landau e efeitos diamagnéticos simultaneamente no estudo de GAM, porém, a partir do modelo girocinético. Efeitos diamagnéticos são causados por termos que envolvem gradientes de densidade e de temperatura provenientes da função Maxwelliana de equilíbrio. O acoplamento entre os harmônicos poloidais, $m= \pm 1$, e as derivadas radiais de quantidades macroscópicas do plasma é responsável pelo aumento no valor da frequência no GAM de alta frequência e pela instabilidade no GAM de baixa frequência. Este tipo de instabilidade, que é proporcional à frequência diamagnética de elétrons e à razão entre os gradientes de temperatura e de densidade, é mais propenso a ocorrer em posições radiais em que o fator segurança é alto. Modos geodésicos são fracamente amortecidos devido a um mecânismo não colisional conhecido por amortecimento de Landau, o qual é causado pela interação entre a onda eletrostática e partículas carregadas, íons no caso, e a taxa de amortecimento é maior próximo ao centro da coluna de plasma, onde o fator de segurança assume valores mais baixos.

O equilíbrio MHD com rotação foi investigado em três regimes com relação às superfícies magnéticas: isotérmico, adiabático e isométrico. Foi observado que o gradiente de temperatura possui sentido oposto em relação à velocidade de rotação poloidal apenas no regime isométrico. Ao considerar equilíbrio com rotação e superfícies magnéticas isotérmicas e incluir fluxo de calor na equação da energia, observamos que ZF apresentam frequência não-nula, a qual é proporcional à velocidade de rotação poloidal e inversamente proporcional ao fator de segurança.

Como direções futuras ressaltamos que é importante considerar efeitos eletromagnéticos, estudar automodos geodésicos e incluir o efeito de partículas aprisionadas para o desenvolvimento da física de ZF e GAM. Tal desenvolvimento beneficiará tanto a área de transporte em tokamaks como a área de diagnósticos, na qual a obtenção do perfil radial da temperatura de íons e do fator de segurança é um dos objetivos. Nesta área, um novo tipo de diagnóstico conhecido como espectroscopia em modos acústicos geodésicos está sendo desenvolvido baseado no estudo de automodos.

Palavras chaves: Modos acústicos geodésicos, GAM, efeitos diamagnéticos, efeitos de deriva, amortecimento de Landau, fluxos zonais, ZF, espectroscopia em GAM. 


\begin{abstract}
Due to the important role in drift wave turbulence and applications for plasma diagnostic purposes, the investigation of zonal flows (ZF) and associated geodesic acoustic modes (GAM) has arisen much attention in the plasma physics literature. In this thesis, first we consider equilibrium poloidal and toroidal rotation effects on these modes using the ideal MHD model, then we investigate diamagnetic effects on GAM using a two fluid model that includes parallel ion viscosity, and, in the final step, we include both Landau damping and diamagnetic effects on the study of GAM within the framework of the gyrokinetic model. By diamagnetic effects we mean the density and temperature radial gradients terms coming from the equilibrium Maxwellian distribution function. The effects caused by the coupling between the $m= \pm 1$ poloidal harmonics and the radial derivatives of equilibrium macroscopic quantities are responsible for an increase in the frequency value of the high frequency GAM and for an instability in the low frequency GAM. This instability, which is proportional to the electron drift frequency and the ratio between ion temperature and density gradients, are more likely to occur in radial positions where the safety factor is high. We observe that geodesic modes are slowly damped by a collisionlees mechanism known as Landau damping which is caused by the wave particle interaction between the eletrostatic potential and the íons. This damping is enhanced near the center of the plasma column, where the safety factor has lower values.

Equilibrium MHD with plasma rotation were investigated in three regimes regarding the magnetic surfaces: isotherm, adiabatic and isometric. It is found that the temperature gradient has opposite directions compared to the poloidal rotation only for the isometric regime. By considering equilibrium rotation with isotherm magnetic surfaces and including heat flux we observed that ZF has a non-zero frequency which is proportional to the poloidal velocity and the inverse of the safety factor.

For future directions we point out that electromagnetic effects, geodesic eigenmodes and trapped particles physics should be important for the development of the ZF and GAM physics, either in the area of anomalous transport caused by drift wave turbulence or for diagnostic purposes for obtaining the radial profile of the ion temperature and the safety factor. In this area, a new kind of diagnostic known as geodesic acoustic mode spectroscopy is being developing based on the study of eigenmodes.
\end{abstract}

keywords: Geodesic Acoustic Modes, GAM, diamagnetic effects, drift effects, Landau damping, zonal flows, ZF, GAM spectroscopy. 


\section{Sumário}

1 Introdução 1

1.1 Energia para futuras gerações . . . . . . . . . . . . . . 1

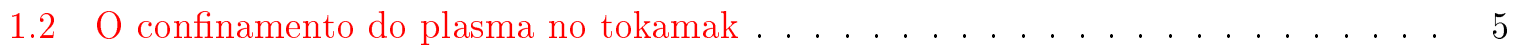

1.2.1 O princípio de confinamento magnético . . . . . . . . . . . 5

1.2.2 Degradação do confinamento em tokamaks . . . . . . . . . . . . 7

1.3 Motivação para o estudo de modos geodésicos e resultados obtidos . . . . . . . 8

1.4 Frequência dos modos acústicos geodésicos $(\mathrm{GAMs}) \ldots \ldots \ldots$. . . . . . . 12

1.5 Organização desta tese . . . . . . . . . . . . . . . . . . . 13

1.5 .1 Capítulos . . . . . . . . . . . . . . . 13

1.5 .2 Apêndices . . . . . . . . . . . . . . . . . . . . . . 14

2 Física de tokamaks $\quad 15$

2.1 Comprimentos e tempos característicos do plasma . . . . . . . . . 16

2.2 Campo magnético e equilíbrio no tokamak . . . . . . . . . . . . . . . . . 19

2.3 Difusão e transporte em tokamaks . . . . . . . . . . . . . . . . . . . 22

2.4 Movimento de partículas e velocidade do centro guia . . . . . . . . . . 26

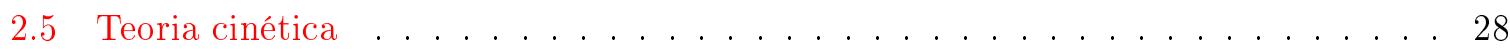

2.5.1 Análise da equação de Boltzmann . . . . . . . . . . . . . . . . . 28

2.5 .2 A equação girocinética . . . . . . . . . . . . . . . . . . 31

2.5 .3 Equação cinética de deriva . . . . . . . . . . . . . . . . . . 33

2.6 Teoria de fluidos . . . . . . . . . . . . . . . . . . . 33

2.6.1 Teoria de dois fluidos e as equações de Braginskii . . . . . . . . . . . . . 33 
2.6.2 Teoria da magneto-hidrodinâmica (MHD) ideal . . . . . . . . . . . . . . 37

3 Modelo de fluido para fluxos zonais e modos acústicos geodésicos

3.1 Modelo da magnetohidrodinâmica (MHD) ideal . . . . . . . . . . . . . . . . . 44

3.2 Equilíbrio com rotação . . . . . . . . . . . . . . . . . . . . . . 45

3.2 .1 Rotação toroidal . . . . . . . . . . . . . . . . . . . . 50

3.2 .2 Rotação poloidal e toroidal . . . . . . . . . . . . . . . . . 51

3.3 Sistema de equações perturbadas e relação de dispersão . . . . . . . . . . . . . 54

3.4 Fluxos zonais $(\mathrm{ZF})$ e modos acústicos geodésicos $(\mathrm{GAM}) \ldots \ldots \ldots \ldots$

3.5 Efeito de rotação nos GAM e ZF . . . . . . . . . . . . . . . . . . . . 62

3.5.1 Efeito da rotação toroidal . . . . . . . . . . . . . . . . . . 65

3.5.2 Efeito da rotação poloidal e toroidal . . . . . . . . . . . . . . . . 65

3.6 Discussão sobre o índice adiabático . . . . . . . . . . . . . . . . 67

3.7 Modelo de dois fluidos com viscosidade paralela . . . . . . . . . . . . . 68

3.7.1 Efeito de anisotropia de pressão nos GAM . . . . . . . . . . . . . . . . 69

3.7.2 Efeitos diamagnéticos nos GAM . . . . . . . . . . . . . . . 71

3.8 Discussão sobre GAM eletromagnético ～. . . . . . . . . . . . . . . . 74

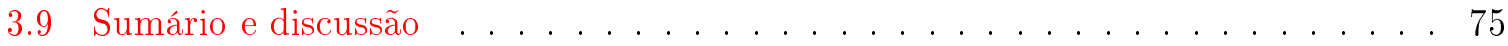

4 Investigação de modos acústicos geodésicos (GAM) pelo modelo girocinético 80

4.1 Estudo de GAM a partir do modelo girocinético . . . . . . . . . . . . . . 80

4.1.1 Limite de fluido com $k_{\|} v_{T_{i}}=0(q \rightarrow \infty) \ldots \ldots \ldots$. . . . . . 84

4.1.2 Limite de fluido com $k_{\|} v_{T_{i}}$ finito $(q \gg 1) \ldots \ldots \ldots 6$

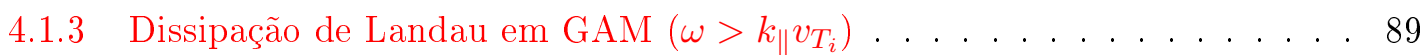

4.2 Discussão sobre aplicações do modelo girocinético na forma mais geral . . . . . . 92

4.3 Efeitos diamagnéticos e amortecimento de Landau em GAM . . . . . . . . . . . . 94

4.3.1 Soluções no limite de fluido . . . . . . . . . . . . . . . . . . . . . . . 97

4.3.2 Efeito cinético em modos geodésicos (amortecimento de Landau) . . . . . 99

4.4 Sumário e discussão . . . . . . . . . . . . . . . . . . . . . . . . 99 
5.1 Modelo de fluidos . . . . . . . . . . . . . . . . . . . . . . . . . 102

5.2 Modelo girocinético . . . . . . . . . . . . . . . . . . . . 105

5.3 Propostas para trabalhos futuros . . . . . . . . . . . . . . . 107

A Constantes e parâmetros do TCABR e ITER 109

A.1 Constantes da Física . . . . . . . . . . . . . . . . . . . . . . . . . 109

A.2 Parâmetros do TCABR e do ITER . . . . . . . . . . . . . . . . . . . . . 110

A.3 Ordens de grandeza de frequências e velocidades características do TCABR . . . 110

B Identidades e relações vetoriais $\quad 111$

B.1 Identidades vetoriais $\ldots \ldots \ldots \ldots \ldots \ldots \ldots$

B.2 Identidades e teoremas fundamentais . . . . . . . . . . . . . . . . . . 112

B.3 Identidades envolvendo o operador $\nabla \ldots \ldots$. . . . . . . . . . . . . . . 112

B.4 Gradiente, Divergente, Rotacional e Laplaciano em coordenadas cilíndricas . . . . 113

B.5 Gradiente, Divergente e Rotacional em coordenadas quasi-toroidais . . . . . . . . 113

B.6 Derivadas de versores em coordenadas cilíndricas . . . . . . . . . . . . . . . . 114

B.7 Derivadas de versores em coordenadas quasi-toroidais . . . . . . . . . . . . . . 114

C Obtenção das expressões analíticas referentes à análise de equilíbrio com rotação

C.1 Relações envolvendo $\mathbf{B}$ e $\mathbf{J}$. . . . . . . . . . . . . . . . . . . . . . . 115

C.2 Relações para $\mathbf{V} \ldots \ldots \ldots$. . . . . . . . . . . . . . . . . 116

C.3 Cálculo de $\nabla \cdot$ q de equilíbrio . . . . . . . . . . . . . . . . 120

D Derivação de fórmulas usadas no capítulo 3

D.1 Relações para B . . . . . . . . . . . . . . . . . . . . . . . . 122

D.2 Cálculo da divergência de $\pi, \mathbf{v}, \mathbf{J}$ e $\mathbf{q} \ldots \ldots \ldots \ldots \ldots \ldots$

D.2.1 Relações para velocidades $(\mathbf{v})$. . . . . . . . . . . . . . . . . . . . . 124

D.2.2 Relações para a densidade de corrente $(\mathbf{j}) \ldots \ldots$. . . . . . . . . . 125

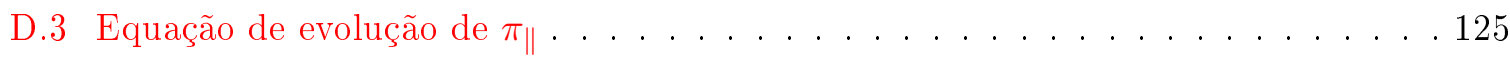


D.4 Aproximação para tokamaks de superfícies magnéticas concêntricas . . . . . . . . 127

D.4.1 Campo magnético de equilíbrio . . . . . . . . . . . . . . . 127

D.4.2 Campo magnético perturbado . . . . . . . . . . . . . 127

D.4.3 Velocidade e densidade de corrente. . . . . . . . . . . . . . . . 128

E Solução iterativa das equações perturbadas da MHD ideal 129

E.1 Equações iniciais e solução de equilíbrio . . . . . . . . . . . . . . . . . . 129

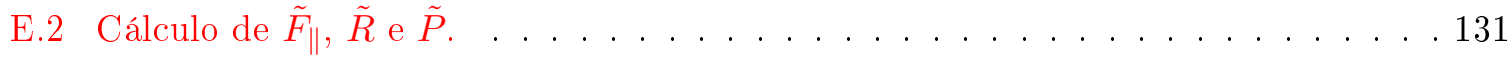

E.2.1 Termos de convecção e derivadas angulares . . . . . . . . . . . . . . 131

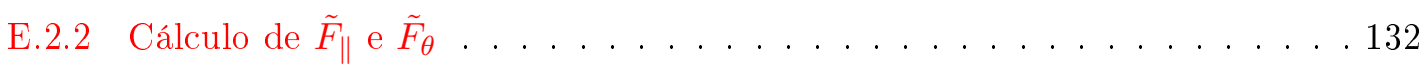

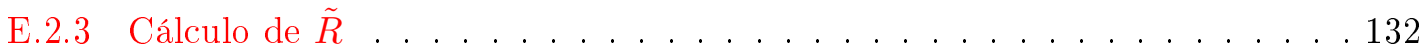

E.2.4 Cálculo de $\nabla \cdot \tilde{\mathbf{q}} \ldots \ldots \ldots \ldots . \ldots \ldots \ldots$

E.2.5 Cálculo de $\tilde{P} \ldots \ldots \ldots \ldots$. . . . . . . . . . . . . . . . . . . . . . . . . . .

E.3 Solução sem rotação (primeira iteração) . . . . . . . . . . . . . . . . . . 134

E.4 Solução com rotação toroidal (segunda iteração) . . . . . . . . . . . . . . . . . 134

E.5 Rotação poloidal e toroidal (terceira iteração) . . . . . . . . . . . . . . . . 136

E.6 Relação de dispersão . . . . . . . . . . . . . . . . . . . . . . . . . . . 139

F Cálculo de integrais da função distribuição $\quad 141$

F.1 Relações envolvendo a distribuição maxwelliana . . . . . . . . . . . . . . . . . . . 141

F.2 Cálculo das integrais na aproximação de fluido . . . . . . . . . . . . . . . 143

F.3 Função dispersão de plasma . . . . . . . . . . . . . . . . . . . . . . . . . . 144

F.4 Cálculo das integrais com efeitos cinéticos . . . . . . . . . . . . . 146

F.5 Obtenção do limite de fluido a partir das integrais com efeitos cinéticos . . . . . . 148

G Participação em eventos científicos $\quad 149$

G.1 Cursos internacionais: . . . . . . . . . . . . . . . . . . . . . . . . 149

G.2 Produção bibliográfica . . . . . . . . . . . . . . . . . . . . . . . . . 149

G.3 Conferências e encontros científicos . . . . . . . . . . . . . . . . 150

Referências Bibliográficas . . . . . . . . . . . . . . . 151 


\section{Lista de Figuras}

1.1 Esquema de obtenção de energia por meio de fusão termonuclear controlada em um futuro reator a fusão baseado no tokamak . . . . . . . . . . . . . . . . . 4

1.2 Esquema de um tokamak . . . . . . . . . . . . . . . . . 6

3.1 Perfil radial da velocidade de rotação de equilíbrio poloidal (esquerda) e toroidal (direita) no tokamaks TCABR . . . . . . . . . . . . . 53

3.2 Dinâmica de modos acústicos geodésicos (GAM) em tokamaks. . . . . . . . . . 61

3.3 Singularidades do denomindador de $D^{(\mathrm{P})}$ para $M_{P} \geq 0 \ldots \ldots \ldots$. . . . . 64 


\section{Lista de Tabelas}

3.1 Frequências normalizadas em diferentes regimes de equilíbrio com rotação toroidal 66

4.1 Frequências típicas normalizadas (por $v_{T_{i}} / R_{0}$ ) relacionadas a efeitos geodésicos, acústicos de íons e diamagnéticos.

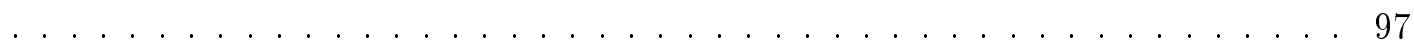

A.1 Constantes físicas pertinentes a esta tese . . . . . . . . . . . . . . . . 109

A.2 Principais parâmetros dos tokamaks TCABR e ITER . . . . . . . . . . . . . 110

A.3 Valor da ordem de grandeza da frequência de giração, da velocidade térmica e do raio de Larmor para o tokamak TCABR . . . . . . . . . . . . . . . . . . 110 


\section{Capítulo 1}

\section{Introdução}

Nesta introdução, inicialmente, discutimos aspectos sobre a questão da produção de energia para o consumo humano e, ainda neste contexto, apresentamos a proposta da fusão termonuclear controlada como meio alternativo para superar a maioria dos problemas da área de energia. O princípio de confinamento do plasma no tokamak e alguns dos principais desafios da física de tokamaks são elucidados em seguida.

Na terceira seção, na qual as principais referências relacionadas ao tema desta tese foram inseridas, apresentamos a motivação para este trabalho, que tem como principal objetivo investigar modos acústicos geodésicos $\left(\mathrm{GAM}^{1}\right)$. Devido a sua importância na área de turbulência e, mais recentemente, na área de diagnósticos, e por estarem presentes no regime de confinamento melhorado, modos de baixas frequências em tokamaks, em especial GAM, têm sido alvo de intensa especulação.

Finalmente, a forma como esta tese está organizada, os assuntos de que trata cada capítulo e o conteúdo dos apêndices são apresentados de forma resumida na última seção.

\subsection{Energia para futuras gerações}

Há algumas décadas atrás, quando o aquecimento global não era considerado um problema em potencial e, além disso, as reservas naturais de petróleo e carvão eram consideradas garantia

\footnotetext{
${ }^{1}$ Geodesic Acoustic Modes
} 
de energia suficiente por um longo período, métodos alternativos de produção de energia, tais como energia solar, eólica, geotérmica, etc... não tinham tanta força para prevalecer na prática. Atualmente, entretanto, a ameaça de possíveis mudanças negativas no planeta, provocadas principalmente pelo aquecimento global, tem sido considerada uma preocupação para cientistas e ambientalistas no mundo inteiro. Ademais, sinais de escassez de recursos naturais e o aumento do consumo de energia, principalmente devido ao desenvolvimento tecnológico e ao aumento da população mundial, mostram-se cada vez mais evidentes, de forma que a busca por formas alternativas de produzir energia com um mínimo de impacto ambiental vem ganhando força em diversos países, inclusive na mídia.

Contudo, é possível, considerando algumas estimativas, que as atuais formas de energia limpa e renováveis não possam suprir a demanda causada pelo aumento do consumo de energia que certamente ocorrerá nas próximas décadas. Por esta razão torna-se imprescindível o desenvolvimento de novos mecanismos de produção de energia e, em particular, da energia por meio de fusão termonuclear controlada, que é uma solução em potencial para o problema. Isto se deve ao fato de que, além de baixo o impacto ambiental causado e, a longo prazo, o custo de obtenção dos combustíveis a serem utilizados na reação de fusão poderá ser reduzido, a grande quantidade de energia que poderá ser produzida de forma sustentável é uma vantagem decisiva. Quanto às questões sobre a produção de resíduos radioativos e ao risco de acidentes nucleares, considerados de suma relevância quando se trata de reatores a fissão nuclear, o tempo de armazenamento destes resíduos para que não causem danos ao meio ambiente é bem menor no caso da fusão e, além disso, o impacto de um improvável acidente não se estenderia a proporções maiores do que o local de instalação do reator, ao contrário do que ocorreu no passado com reatores a fissão. Com base nestes argumentos [1,2], acredita-se que a energia a fusão é a solução mais eficaz no que se refere à produção de grande quantidade de energia de forma sustentável com um mínimo de impacto ambiental. Entretanto há muitos desafios tanto na física como na engenharia não superados ainda que impedem a obtenção de energia por meio de fusão. Com o objetivo de superar estes desafios, pesquisas em diversos dispositivos de confinamento de plasma vêm sendo realizadas ao longo do tempo e, atualmente, acredita-se que o tokamak ${ }^{2}$, inventado por I. Tamm

\footnotetext{
${ }^{2} \mathrm{O}$ nome tokamak é proveniente de "toroidalnaya kamera magnitnaya katushka", que em russo significa
} 
e A. Sakharov [3] na década de 50, é o dispositivo mais propenso a ser utilizado na primeira usina de energia a fusão. Tokamaks localizados em diversas partes do planeta [4] vêm sendo construídos ao longo de décadas, após a segunda guerra mundial, com a finalidade de promover a pesquisa em física de plasmas com o objetivo de superar os desafios que impedem a obtenção de energia por meio de fusão.

O processo para obtenção de energia elétrica por meio fusão nuclear, ilustrado na figura 1.1, consiste em produzir e confinar um plasma constituído por deutério (D) e Trítio (T), isótopos do hidrogênio, mantendo-o a determinados valores de temperatura e de densidade, mas ainda assim, de maneira estável, de forma que possa ocorrer com frequência a seguinte reação:

$$
\mathrm{D}+\mathrm{T} \rightarrow{ }^{4} \mathrm{He}(3.5 \mathrm{MeV})+\mathrm{n}(14.1 \mathrm{MeV}) .
$$

Em uma fase inicial do desenvolvimento de reatores a fusão, por ser uma das reações nucleares mais simples de se realizar, a reação expressa em (1.1) seria utilizada ao invés de outras mais eficientes que, entretanto, necessitam de temperaturas mais elevadas.

Nêutrons energéticos provenientes do plasma, ao atingirem a camada de Lítio (Li), que reveste as paredes internas do tokamak, transfeririam sua energia, na forma de calor, a uma tubulação de água corrente com a produção de vapor a alta pressão como consequência deste processo. Este vapor seria então utilizado para acionar um gerador de eletricidade que, por sua vez, abasteceria a rede elétrica das cidades. A função da camada de Li no tokamak, disposta em uma manta, é fornecer átomos de trítio ao plasma, de acordo com a reação:

$$
{ }^{6} \mathrm{Li}+\mathrm{n} \rightarrow{ }^{4} \mathrm{He}+\mathrm{T}+5 \mathrm{MeV} .
$$

De maneira global, neste processo de várias etapas de transformação de energia, o resultado líquido seria transformar a energia nuclear contida nos átomos de deutério e de trítio em energia elétrica e, liberando como resíduo desta transformação gás Hélio, que poderia ser liberado ao meio ambiente sem o prejuízo de nenhum impacto ambiental, uma vez que este gás é inerte e estável. Apesar da enorme quantidade de energia que poderia ser conseguida com baixas 
quantidades de Deutério e de Lítio, abundantes na natureza, o risco de acidentes nucleares é baixo e o impacto ambiental praticamente inexiste.

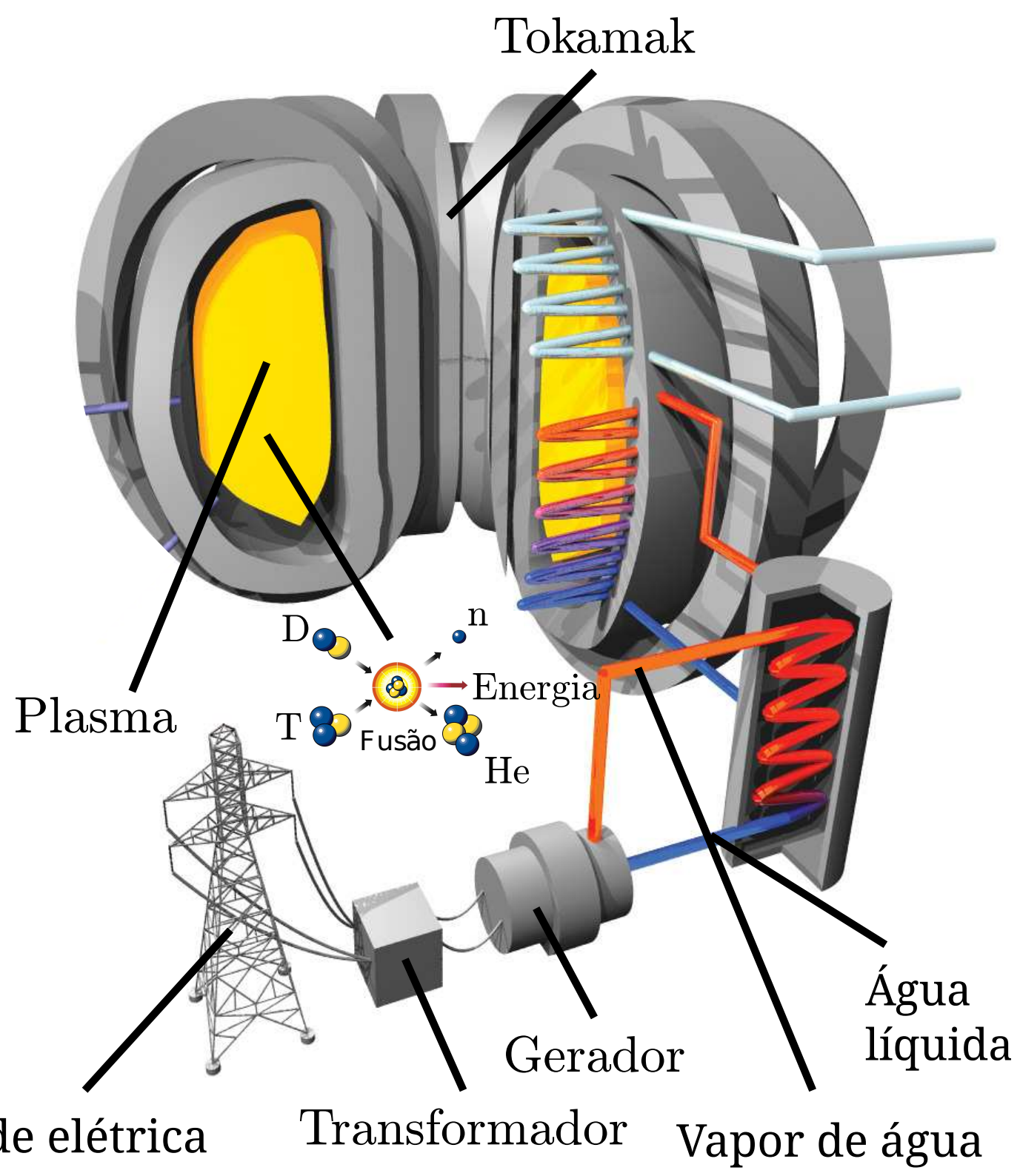

Figura 1.1: Esquema de obtenção de energia por meio de fusão termonuclear controlada em um futuro reator a fusão baseado no tokamak.

Obs: Adaptação a partir das figuras originais provenientes das seguintes fontes:

http://iter.rma.ac.be/en/sustain/FusionPlant/index.php (Acessado em 14/05/2014)

http://www.infoescola.com/quimica/quimica-nuclear/(Acessado em 14/05/2014)

O maior desafio, no entanto, consiste em conseguir manter o plasma confinado nas condições necessárias para que reações de fusão possam ocorrer. Diversas instabilidades e processos de dissipação, que ocorrem durante o processo de confinamento do plasma, impedem que este 
confinamento dure tempo suficiente para que o tokamak possa ser usado como um reator nuclear. Em particular, há o mecanismo de transporte, no qual partículas e energia do plasma são perdidas rapidamente, degradando, assim, o confinamento.

\subsection{O confinamento do plasma no tokamak}

\subsubsection{O princípio de confinamento magnético}

O plasma permanece confinado no tokamak quando ocorre o equilíbrio entre a força devida ao gradiente de pressão cinética e a força magnética ${ }^{3}$, de acordo com a equação

$$
\nabla p=\mathbf{J} \times \mathbf{B},
$$

onde $p$ é a pressão cinética do plasma e $\mathbf{J}=\mu_{0}^{-1} \nabla \times \mathbf{B}$ é a corrente produzida pelo campo magnético (B) e pelo gradiente de pressão. A primeira força, devida à pressão do plasma, é uma consequência natural do comportamento de gás apresentado pelo plasma, o qual é decorrente das frequentes colisões a que estão sujeitas as partículas que compõem este. Em oposição a esta, a força magnética, que age no sentido de confinar o plasma, é produzida pela interação entre o campo magnético e a corrente que percorre o plasma.

No parágrafo que se segue, o mecanismo de confinamento do plasma, a partir da criação deste, bem como os principais campos magnéticos, correntes e dispositivos mais importantes, os quais também são mostrados na figura 1.2.1, são descritos.

Em um primeiro momento, o plasma é produzido a partir da ionização do gás neutro (normalmente hidrogênio ou deutério) contido na câmara de vácuo, que ocorre devido à alta tensão induzida pela transferência da energia contida em um conjunto de capacitores de alta potência para o enrolamento central. Note que o tokamak age como um transformador, onde a coluna de plasma, em forma de anel, atua como enrolamento secundário. Após a ionização do gás, ocorre então uma queda brusca na resistividade deste, momento em que o plasma é produzido, passando a circular, então, uma intensa corrente no plasma, a corrente de plasma $\left(I_{p}\right)$. O plasma

\footnotetext{
${ }^{3}$ É comum também utilizar a formulação em termos da pressão magnética $\left(B^{2} / 2 \mu_{0}\right)$
} 


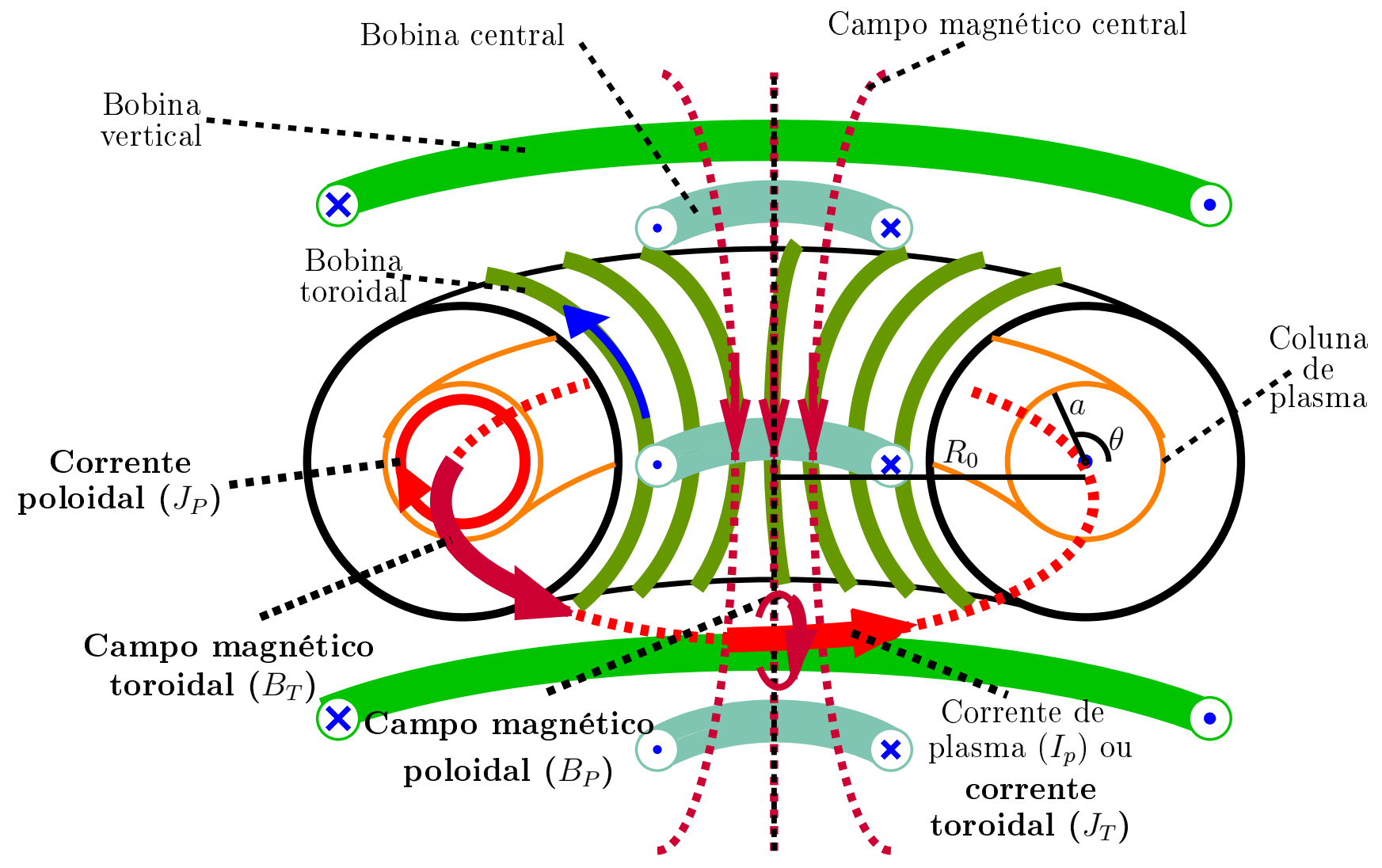

Figura 1.2: Esquema de um tokamak

é então aquecido a temperaturas estelares, condição indispensável para que reações de fusão nuclear, como a mostrada na eq. (1.1), possam ocorrer com frequência. Nesta etapa do processo, para conter o efeito da forte pressão cinética, que aumenta com a temperatura, forçando o plasma a se expandir e dificultando, portanto, o confinamento, a força magnética desempenha um papel essencial. Esta força surge em decorrência da interação entre as correntes que circulam no plasma e o campo magnético nele presente. Para que haja confinamento é necessário que este campo magnético apresente duas componentes: uma na direção toroidal, $B_{T}$, produzida externamente pelo enrolamento toroidal, e a outra na direção poloidal, $B_{P}$, criada pela própria corrente de plasma. Além da corrente de plasma, na direção toroidal, o comportamento coletivo do plasma permite o surgimento de uma outra corrente neste, porém, na direção poloidal. Esta corrente surge em consequência da deriva diamagnética devido à existência de um gradiente (radial) de densidade. Por fim, há ainda um outro efeito indesejável ao confinamento, o qual 
justifica a utilidade das bobinas verticais. A coluna de plasma tende a aumentar seu raio maior em direção à câmera de vácuo, similar ao que ocorre em um anel de corrente imerso em um campo magnético [5]. Este problema, porém, pode ser corrigido por meio de uma corrente controlável que atravessa as bobinas verticais, as quais foram implementadas com o objetivo de criar um campo magnético vertical capaz de controlar a posição do plasma.

Na literatura há inúmeras referências a respeito da teoria de tokamaks, nas quais detalhes importantes, do ponto de vista físico, sobre o processo pelo qual o plasma é confinado em tokamaks são apresentados. Uma exposição didática, simples e qualitativa, que trata não somente da física de tokamaks mas também de muitas questões relacionadas à área de energia, pode ser encontrada em [6]. Para um estudo mais aprofundado, que envolve detalhes algébricos, algumas das referências mais tradicionais, [4,5,7], são recomendadas.

\subsubsection{Degradação do confinamento em tokamaks}

Após o confinamento do plasma em um primeiro momento, há ainda outros obstáculos, dos quais mencionamos apenas dois, a serem vencidos para que se possa tornar uma realidade e, portanto, proporcionar um enorme avanço científico à possibilidade de obtenção de energia por meio de fusão nuclear.

Primeiramente, há de se considerar a impossibilidade de o tokamak agir como transformador por muito tempo, o que manteria a corrente de plasma (essencial para o confinamento), pois, para isso, o valor da tensão aplicada ao enrolamento central teria que crescer constantemente por um longo período, o que é impossível do ponto de vista prático. Entretanto é essencial a presença da corrente de plasma para o confinamento, o que requer uma solução alternativa para o problema. Constitui uma das linhas de pesquisa em plasmas de tokamaks o desenvolvimento de mecanismos capazes de manter a corrente de plasma e aquecer o plasma. Tais mecanismos se baseiam na excitação de ondas no plasma e na transferência de partículas neutras a este por meio de dispositivos auxiliares. Em particular, com a descoberta de ondas de Alfvén [8], a sua utilização na geração de corrente e no aquecimento do plasma em sistemas de confinamento magnético, se tornou uma área de pesquisa ativa em muitos tokamaks e, em especial, no 


\section{$\operatorname{TCABR}^{4}[9]$.}

Um outro grande obstáculo para a área de plasmas de fusão é o transporte de partículas, energia e calor que ocorre no plasma, degradando rapidamente o confinamento. Especial ênfase deve ser dada ao transporte anômalo (ou turbulento), o qual é muito maior do que o transporte clássico que ocorre em gases neutros. Entretanto, com a descoberta de um novo regime de confinamento, também conhecido como modo $\mathrm{H}^{5}$ [10], houve uma significativa contribuição para o desenvolvimento de tokamaks, em especial o $\operatorname{ITER}^{6}$ [1]. No modo H, forma-se um forte gradiente de pressão em certa região do plasma, também conhecido como barreira de transporte $\left(\mathrm{TB}^{7}\right)$, pois o transporte turbulento é significativamente reduzido nesta região. Com relação ao processo de turbulência no plasma, que contribui significativamente para o transporte anômalo, descobriu-se a ocorrência de determinados modos no plasma, os modos acústicos geodésicos (GAM), também conhecidos por "fluxos zonais (ZF) de alta frequência" (porém de frequência bem iferior a frequência de giração de íons), que são capazes de suprimir um tipo especial de turbulência: turbulência de ondas de deriva $\left(\mathrm{DWT}^{8}\right)$. A excitação e identificação experimental de GAM em tokamaks, bem como a compreensão do processo de auto-organização responsável pela supressão de turbulência, tem sido alvo de intensa pesquisa teórica e experimental [11,12].

\subsection{Motivação para o estudo de modos geodésicos e resultados obtidos}

Modos geodésicos de baixas frequências, em especial GAM, têm sido alvo de intensa investigação, teórica e experimental, não somente devido ao seu papel na supressão de transporte turbulento em tokamaks [11,13], mas também devido a sua relação com auto-modos de Alf-

\footnotetext{
${ }^{4}$ O Tokamak Chauffage Alfvén Brésilien (TCABR) fica localizado no Instituto de Física (IF) da Universidade de São Paulo (USP), no Brasil.

${ }^{5}$ High (confinement)

${ }^{6}$ O International Thermonuclear Experimental Reactor (ITER) é o primeiro reator a fusão (em construção ainda) baseado na tecnologia de tokamaks. Localizado em Cadarashe, na França, ele esta sendo projetado para produzir 500 MW de potência.

${ }^{7}$ Transport Barrier

${ }^{8}$ Drift wave turbulence
} 
vén induzidos pela pressão $\left(\mathrm{BAE}^{9}\right)$ [14-18]. A observação da atividade magneto-hidrodinâmica $\left(\mathrm{MHD}^{10}\right)$ devido a modos geodésicos pode ter também aplicações diagnósticas, especialmente no que se refere à espectroscopia em MHD [19]. O estudo de BAE é de fundamental importância na investigação da turbulência de fundo, na geração de turbulência e, em espectroscopia MHD, para diagnosticar o fator de segurança, $q$, [20,21]. No que se refere à verificação experimental dos GAM, importantes experimentos em diferentes tokamaks [22-25] não só confirmaram a sua existência mas também revelaram aspectos cruciais com relação a sua localização e intensidade.

A principal motivação desta tese reside no objetivo de contribuir, ainda que indiretamente, com resultados teóricos, qualitativos e quantitativos, que ajudem a entender melhor o comportamento de modos de baixas frequências e o mecanismo de supressão de turbulência por ondas de deriva em tokamaks. O conhecimento físico sobre este mecanismo, bem como o estudo de transporte anômalo pertencem a um conjunto de desafios científicos a serem superados para o desenvolvimento do primeiro reator a fusão nuclear e, por isso, podem desempenhar um papel de importância econômica, social e ecológica em escala global no futuro.

Neste sentido, por considerarmos um tema muito importante, investigamos diversos efeitos nos GAM [26], os quais, pelo fato de estarem diretamente ligados à DWT, têm sido alvo de intensa pesquisa teórica e experimental em tokamaks. Segue abaixo uma breve discussão sobre estes efeitos e os principais resultados decorrentes que obtivemos:

- Rotação de equilíbrio: Causada pela presença de um campo elétrico radial de equilíbrio e gradientes de temperatura e densidade, tem sido investigada principalmente devido a sua ocorrência durante a formação da barreira de transporte (TB) no regime de confinamento melhorado (ou modo H) [10]. Investigamos a influência de rotação poloidal e toroidal na frequência dos modos GAM (capítulo 3) a partir do modelo da MHD ideal (resistividade nula) para um fluido. Foram considerados três tipos de equilíbrio: adiabático, isotérmico e isométrico (ou isocórico), os quais são descritos abaixo:

- Equilíbrio adiabático: Por estar no regime característico da propagação de ondas de som, não somente em plasma mas também em fluidos neutros, assim como

\footnotetext{
${ }^{9}$ Beta-Alfvén Eigen-modes

${ }^{10}$ Magnetohydrodynamics
} 
esperado, influencia diretamente a frequência dos GAM, no sentido de aumentar esta, conforme a eq. (3.121), não importando o sentido da rotação. Quando existe um fluxo poloidal de equilíbrio, há uma segunda solução, que corresponde ao ramo sonoro de íons, e cuja frequência normalizada, da ordem do inverso do fator de segurança $\left(q^{-1}\right)$ em relação à frequência dos GAM, se encontra no valor intermediário entre o ramo dos GAM e dos ZF. Este último ramo, de frequência nula normalmente, não tem sua frequência alterada exclusivamente para este equilíbrio, o que indica que, do ponto de vista teórico, o fluxo de calor (q) desempenha um papel importante no mecanismo de formação dos ZF.

- Equilíbrio isotérmico: O aparecimento de ZF estáveis, porém de frequência finita, quando há rotação poloidal, é a principal consequência deste tipo de equilíbrio, no qual fluxo de calor perpendicular, devido ao gradiente radial de temperatura, deve ser considerado.

- Equilíbrio isométrico (ou isocórico): Permite a presença de fluxos zonais (ZF) instáveis, mesmo para o caso de rotação exclusivamente toroidal [27], o que, de certa forma, confirma a relação destes com fluxos incompressíveis no plasma. Uma das inovações desta tese foi investigar o efeito do equilíbrio isométrico com rotação poloidal nos ZF.

Em resumo, ao considerarmos diferentes equilíbrios com rotação encontramos três soluções, com valores de frequências distintos e característicos de fenômenos físicos diferentes que ocorrem no plasma. Tais fenômenos decorrem de características peculiares do plasma e do tokamak, as quais estão relacionadas à curvatura geodésica do campo magnético no tokamak, à propagação de ondas típica de fluidos, ao importante parâmetro do tokamak conhecido como fator de segurança e à condição de compressibilidade ou incompressibilidade que o plasma assume.

- Efeitos diamagnético (ou efeitos de derivas ${ }^{11}$ ): Efeitos causados por gradientes de densidade e temperatura de equilíbrio em modos geodésicos de baixas frequências,

\footnotetext{
${ }^{11}$ Drift effects
} 
foram investigados nesta parte (capítulos 3 e 4). Nossos objetivos são apresentar os mecanismos físicos envolvidos nas oscilações dos GAM, expor o conteúdo da forma mais simples e compreensível possível e incluir efeitos cinéticos. Para atingir tais objetivos foram utilizados dois modelos, o modelo de fluidos e o modelo giro-cinético, de forma que a consistência física proporcionada pelo modelo de fluido e a maior generalidade do modelo cinético contribuam para nossa meta. Ao considerar o efeito da deriva diamagnética e o efeito de rotação de equilíbrio nos GAM, pudemos observar que estes efeitos estão relacionados. Aspectos relativos a esses dois modelos e os resultados decorrentes de seu uso são mostrados a seguir:

- Modelo de dois fluidos: Este modelo é diferente do modelo da MHD ideal, considerado anteriormente, por duas razões: primeiro, os dois fluidos característicos, de íons e de elétrons, são considerados em regimes distintos e, segundo, devido à interação entre as partículas do plasma e o campo magnético macroscópico presente neste, a diferença entre a pressão paralela e a perpendicular (com relação ao campo magnético de equilíbrio), deve ser considerada, com a inclusão do tensor de viscosidade paralela [28]. Esta consideração permite obter resultados condizente com a teoria cinética [29], a qual leva em consideração o efeito da anisotropia de pressão, embora de forma não tão clara. Uma forma alternativa capaz de descrever o efeito da anisotropia é através do formalismo CGL [30]. Em favor da simplicidade e do didatismo, na exposição do capítulo 3 consideramos o limite $q \rightarrow \infty$, enfatizando, assim, apenas duas questões: o efeito dos gradientes de densidade e de temperatura nos modos correspondentes ao ramo geodésico (GAM) e ao ramo de mais baixa frequência $(\mathrm{ZF})$ e, devido à anisotropia da pressão perturbada, a correção para o coeficiente adiabático efetivo de íons na expressão para a frequência dos GAM.

- Modelo giro-cinético: Tem como metodologia, assim como qualquer modelo cinético, obter a função distribuição das partículas que compõem o plasma a partir da resolução da equação de Vlasov (equação cinética sem o termo de colisões) [31]. Porém, especificamente para este modelo, esta equação é desenvolvida antes de ser 
resolvida a partir da teoria giro-cinética [32], que é apresentada no capítulo 2 e aplicada, na forma deste modelo, no capítulo 4. Com a utilização desse modelo para incluir efeitos diamagnéticos em modos de baixas frequências, obtivemos três soluções similares às obtidas pela incorporação de rotação de equilíbrio no modelo da MHD ideal (capítulo 3), de forma que propomos, nesta tese, uma alternativa, em princípio, para o estudo de efeitos da rotação de equilíbrio em modos de baixas frequências, quando este estudo for complexo em modelos cinéticos. A solução no ramo sonoro, obtida quando se considera $q$ finito, assim como o amortecimento de Landau dos modos pertencentes aos três ramos foram obtidos no capítulo 4 . Entre as principais consequências causadas pelo amortecimento de Landau, está a deformação da função distribuição de íons e a diminuição do potencial eletrostático perturbado com o tempo, impactando negativamente no confinamento com a extin-

ção dos GAM. Descoberto por L. D. Landau [33], o mecanismo de amortecimento ou crescimento exponencial de uma onda eletromagnética, mesmo em plasmas não colisionais, também conhecido como amortecimento de Landau, é uma consequência da interação onda-partícula que ocorre no plasma devido à presença de partículas com velocidades próximas às da velocidade de fase da onda.

\subsection{Frequência dos modos acústicos geodésicos (GAMs)}

A primeira expressão analítica para a frequência dos GAM [26], obtida por N. Winsor et. al. em 1968, foi desenvolvida a partir do modelo da MHD ideal, no qual se considera o plasma como um fluido único de índice adiabático $\gamma=5 / 3$, pode ser escrita como:

$$
\omega_{\text {gam }(\mathrm{MHD})}^{2}=\left(2+\frac{1}{q^{2}}\right) \frac{\gamma T}{m_{i}}
$$

onde $T=T_{i}+T_{e}$ é a temperatura do plasma, $T_{i}$ e $T_{e}$ são, respectivamente, a temperatura de íons e de elétrons e $m_{i}$ é a massa dos íons (no caso de plasma de hidrogênio).

Posteriormente, em estudos hidrodinâmicos [34], estes modos também foram encontrados 
e, após algumas décadas, a partir da teoria cinética [18,29,35], considerando efeitos adicionais (explicado ao longo desta tese), a expressão cinética obtida para a frequência dos GAM foi:

$$
\omega_{\text {gam }(\mathrm{K})}^{2}=2\left(\gamma_{i}^{(\mathrm{ef})}+\gamma_{e} \frac{T_{e}}{T_{i}}+\mathcal{O}\left(q^{-2}\right)\right) \frac{T_{i}}{m_{i}}
$$

onde $\gamma_{i}^{(\text {ef })}=7 / 4$ é o índice adiabático efetivo para íons e $\gamma_{e}=1$ é o índice adiabático para elétrons. Uma derivação alternativa, [36], a partir da teoria de dois fluidos, considerando íons no regime de fluido $\left(\gamma_{i}=5 / 3\right)$ e elétrons no regime adiabático e isotérmico, com $\gamma_{e}=1$, mostrou ser possível recobrar o resultado cinético (eq. (1.5)) a partir da teoria de dois fluidos. Em tal resultado a diferença entre os coeficientes adiabáticos efetivos de íons e de elétrons se deve à enorme diferença entre as massas destas duas espécies, a qual faz com que a resposta dos elétrons às perturbações seja imediata enquanto os íons, por responderem mais lentamente, ficam sujeitos ao efeito da inomogeneidade da pressão devido a presença do campo magnético. $\mathrm{O}$ efeito da anisotropia de pressão em questão pode ser descrito por meio do tensor de viscosidade paralela $\left(\boldsymbol{\pi}_{\|}\right)$nas equações de fluido $[28,36,37]$, conforme explicado nos capítulos 2 e 3.

\subsection{Organização desta tese}

\subsubsection{Capítulos}

Esta tese é composta por 5 capítulos e 8 apêndices cujos assuntos são sucintamente descritos a seguir. No capítulo 2 os principais modelos físicos, provenientes da teoria de fluidos e teoria cinética aplicadas a plasmas magnetizados, são revisados, frequências e comprimentos característicos fundamentais e parâmetros de plasmas em tokamaks são definidos e, por fim, um breve resumo sobre a teoria de transporte em tokamaks é apresentado. A generalidade do conteúdo, que precede a especificidade dos próximos capítulos, é uma característica deste capítulo que tem por objetivo a revisão de conceitos fundamentais importantes. Em seguida, a partir do modelo da MHD ideal e do modelo de dois fluidos, no capítulo 3, apresentamos o estudo de modos acústicos geodésicos (GAM) e fluxos zonais (ZF). Rotação de equilíbrio e efeitos diamagnéticos são considerados nesse capítulo. Já a investigação cinética, principal tema desta tese, de efeitos 
diamagnéticos e a influência do amortecimento de Landau nos GAM são o conteúdo do capítulo 4. Por fim, no (último) capítulo 5 apresentamos as conclusões científicas desta tese, propostas para continuação da presente linha de pesquisa e breves projetos para trabalhos futuros.

\subsubsection{Apêndices}

O apêndice A, que foi designado para fins de consulta, contem as constantes fundamentais da física pertinentes a esta tese e medidas das principais grandezas de tokamaks de nosso interesse: TCABR e ITER. Identidades importantes, deduções de expressões úteis e longos cálculos algébricos, utilizados principalmente no capítulo 3, são apresentados nos apêndices B - E. Referente ao capítulo 4, segue o apêndice F, que apresenta a dedução de longas expressões e o cálculo de integrais relacionadas à função Maxwelliana e à função dispersão de plasma. Finalmente, no apêndice G, eventos (conferências, encontros, escolas, etc...), colaborações e publicações realizados durante o período de pós-graduação (Mestrado e Doutorado), na área de física de plasmas, são resumidamente descritos. 


\section{Capítulo 2}

\section{Física de tokamaks}

Este capítulo destina-se à discussão de alguns dos tópicos da física de tokamaks, cujo conteúdo será aplicado aos modos específicos de que tratamos nos próximos capítulos. Apresentamos um texto de referência, de caráter mais geral, no sentido em que as teorias aqui tratadas, já bem estabelecidas na área de física de plasmas, são capazes de descrever inúmeros processos e mecanismos físicos que ocorrem em plasmas. Uma descrição e as definições de grandezas fundamentais do plasma, tais como comprimentos e tempos característicos, como ponto de partida para este capítulo, antecedem temas de fundamental importância para a compreensão desta tese, os quais se referem a estrutura do campo magnético e ao mecanismo de transporte de partículas e de energia em tokamaks. A teoria cinética de partículas carregadas e a formulação macroscópica de fluidos, descrita em termos de modelos utilizados posteriormente a este capítulo, são apresentados em seguida.

Por questões de simplicidade, consideramos apenas plasmas de hidrogênio, não levando em conta reações de fusão, de forma que $Z=1$ é adotado ao longo de toda a tese. O plasma é composto por apenas dois (duas) fluidos (espécies de partículas), indexados(as) por $\alpha=i, e$ (íons, elétrons). Também, como é comum em grande parte da literatura em física de plasmas, adotamos a prática de suprimir a constante de Boltzmann $(k)$, cujo valor é mostrado no apêndice A, de forma que a substituição: $k T \rightarrow T$ foi utilizada ao longo desta tese. 


\subsection{Comprimentos e tempos característicos do plasma}

A seguir, discutimos e definimos alguns dos comprimentos e tempos característicos (ou seus inversos, as frequências características) presentes em plasmas de tokamak. Uma discussão mais detalhada dos assuntos tratados aqui é apresentada por F. F. Chen [38].

Iniciamos nossa discussão pela frequência ciclotrônica, a qual pode ser definida ${ }^{1}$ como

$$
\omega_{c_{\alpha}}=\frac{e B}{m_{\alpha}},
$$

onde $e$ é a carga elementar, $m_{\alpha}$ é a massa da partícula do tipo $\alpha$ e $B$ é o campo magnético. A frequência ciclotrônica é uma medida média da rapidez do movimento das partículas em torno das linhas de força. É importante notar que, como $m_{e} \ll m_{i}, \omega_{c_{e}}=\left(m_{i} / m_{e}\right) \omega_{c_{i}} \gg \omega_{c_{i}}$.

Adotando a concepção de fluidos de íons e de elétrons, caracterizados por temperaturas próprias, $T_{i}$ e $T_{e}$, é conveniente definir a velocidade térmica ${ }^{2}$ :

$$
v_{T_{\alpha}}^{2}=\frac{2 T_{\alpha}}{m_{\alpha}},
$$

onde $v_{T_{e}} \gg v_{T_{i}}$, pois normalmente $T_{i} \sim T_{e}$ e $v_{T_{e}}=\left(T_{e} / T_{i}\right)\left(m_{i} / m_{e}\right) v_{T_{i}}$.

A velocidade térmica e a frequência ciclotrônica se relacionam por meio de uma grandeza conhecida como raio de giração ou raio de Larmor, que representa o comprimento característico dos raios das órbitas de partículas em torno das linhas de força (ou linhas de fluxo), o qual é definido como:

$$
\rho_{\alpha}=\frac{v_{T_{\alpha}}}{\omega_{c_{\alpha}}} .
$$

Como $\rho_{e} \sim \sqrt{m_{e} / m_{i}} \rho_{i} \ll \rho_{i}$, o efeito de raio de Larmor finito $\left(\mathrm{FLR}^{3}\right)$ para elétrons não desempenha um papel importante em modos de baixas frequências e, por esta razão, consideramos

\footnotetext{
${ }^{1}$ Alguns autores adotam a definição alternativa: $\omega_{c_{\alpha}}=e_{\alpha} B / m_{\alpha}$, de forma que, para elétrons, $e_{e}=$ $-e$, a frequência ciclotrônica torna-se negativa. Ao optarmos pela convenção de frequências sempre positivas, ressaltamos, entretanto, a necessidade de maior atenção nos cálculos algébricos.

${ }^{2}$ Definimos a temperatura em termos da energia cinética média, $\overline{\mathcal{K}_{\alpha}}=m_{\alpha} v_{T_{\alpha}}^{2} / 2=T_{\alpha}$, porém a definição $v_{T_{\alpha}}^{2}=T_{\alpha} / m_{\alpha}$ também é bastante empregada na literatura.

${ }^{3}$ Finite Larmor Radius
} 
$\rho_{e} \sim 0$ nos capítulos 3 e 4.

Além da velocidade térmica, há duas outras velocidades de particular interesse para o estudo de modos de baixas frequências em plasmas magnetizados. A primeira delas, depdendente da densidade de partículas $\left(n_{i} \approx n_{e}\right)$, é a velocidade de Alfvén,

$$
c_{A} \approx \frac{B}{\sqrt{\mu_{0} n_{i} m_{i}}}
$$

presente no estudo de ondas de Alfvén $\left(\mathrm{AW}^{4}\right)$ [8], que possui importantes aplicações em plasmas, entre elas a determinação da geometria do campo magnético [39]. Estes tipos de onda, que possuem uma ampla gama de classificação, surgem devido a perturbações do campo magnético, que também são referidas e classificadas na literatura como tensão (compressão ou torção das linhas de força). A segunda velocidade de interesse, que é bastante utilizada ao longo desta tese, é a velocidade de som no plasma, que se relaciona à temperatura, densidade $(\rho)$ e pressão $(p)$ por:

$$
c_{s} \approx \sqrt{\frac{\gamma_{i} T_{i}+\gamma_{e} T_{e}}{m_{i}}}=\sqrt{\frac{\gamma p}{\rho}} \sim v_{T_{i}}
$$

onde $\gamma_{i}$ e $\gamma_{e}$ são os coeficientes adiabáticos de íons e de elétrons. Devido a natureza da massa destas partículas, os valores mais razoáveis para estes coeficientes são $\gamma_{i}=5 / 3$ e $\gamma_{e}=1$. O uso de $\gamma$, o índice adiabático total, é feito dentro do contexto da teoria de um fluido, a qual é discutida mais adiante em 2.6.2.

A razão quadrática entre as velocidades de som e de Alfvén possui a mesma ordem de grandeza de um importante parâmetro para o confinamento de plasmas em tokamaks, o fator beta, que é definido como a razão entre as pressões cinética $(p)$ e magnética $\left(B^{2} / 2 \mu_{0}\right)$, ou seja,

$$
\frac{c_{s}^{2}}{c_{A}^{2}} \sim \frac{\beta}{2 \gamma}, \quad \beta=\frac{2 \mu_{0} p}{B^{2}}
$$

Em muitos modelos, como os que apresentamos nesta tese, considera-se regimes de baixa pressão, caracterizado por $\beta=\mathcal{O}\left(\varepsilon^{2}\right)$, onde $\varepsilon=r / R_{0}$ é a razão entre a posição radial e o raio

\footnotetext{
${ }^{4}$ Alfvén Waves
} 
maior do tokamak. Em tais regimes, no modelo MHD ideal é justificável desprezar perturbações magnéticas no estudo de modos de baixas frequências [26, 40, 41]. Também é pertinente considerar, neste tipo de estudo, a condição de quasi-neutralidade, a qual se aplica a fenômenos de comprimentos característicos muito maiores do que o comprimento de Debye, que pode ser definido como:

$$
\lambda_{D_{\alpha}}^{2}=\frac{\varepsilon_{0} T_{\alpha}}{n_{\alpha} e^{2}}
$$

Em comprimentos menores do que $\lambda_{D_{\alpha}}$ ocorrem oscilações de elétrons/íons em resposta à presença de campo elétrico e o plasma deixa de ser neutro localmente. A frequência destas oscilações é conhecida como frequência de plasma e, neste contexto, é definida como:

$$
\omega_{p_{\alpha}}^{2}=\frac{n_{\alpha} e^{2}}{\varepsilon_{0} m_{\alpha}}
$$

Note que $\lambda_{D_{\alpha}} \omega_{p_{\alpha}} \sim v_{T_{\alpha}}$, de forma que para frequências $\omega \sim v_{T_{i}} / R_{0}$, como $\lambda_{D_{\alpha}} \ll R_{0}$, $\omega \ll \omega_{p_{\alpha}}$ e, neste caso, aplica-se a condição de quasi-neutralidade.

A seguir, uma breve discussão sobre os principais tempos (frequências) característicos(as) referentes a processos de colisões é apresentada. Em colisões Coulombianas, conforme os modelos utilizados em [42-45], provenientes da teoria cinética de gases, espera-se que a frequência de colisões elétron-1́on seja $\nu_{\mathrm{ei}} \sim n_{i} \sigma v_{T_{e}}$, onde $\sigma=\pi b^{2}$, é a seção de choque transversal e $b$ é o parâmetro de impacto. A maior dificuldade na determinação desta frequência reside no cálculo de $b$. Como referência, adotamos, para o tempo caracteristico de colisão íon-íon e elétroníon, os valores mostrados em [45], que, quando considerada a condição de quasi-neutralidade $\left(n_{i} \approx n_{e}=n\right)$, são dados respectivamente por:

$$
\tau_{\mathrm{ii}}=\frac{12 \pi^{3 / 2} \varepsilon_{0}^{2}}{\ln \Lambda e^{4} n} m_{i}^{1 / 2} T_{i}^{3 / 2}, \quad \tau_{\mathrm{ei}}=\frac{1}{\sqrt{2}} \frac{m_{e}^{1 / 2}}{m_{i}^{1 / 2}} \frac{T_{e}^{3 / 2}}{T_{i}^{3 / 2}} \tau_{\mathrm{ii}} .
$$

Nestas expressões, $\Lambda \sim n \lambda_{D}^{3}$ é o número médio de partículas dentro de uma esfera de Debye e $\ln \Lambda$ é um termo conhecido como logaritmo coulombiano [31,39,45], cujo valor numérico se encontra entre 10 e 30 . 
Neste contexto, o maior dos tempos característicos é o período de colisão íon-elétron, $\tau_{i e} \sim \tau_{\text {eq }}$, onde

$$
\tau_{\mathrm{eq}}=\frac{m_{i}}{2 m_{e}} \tau_{\mathrm{ei}}
$$

dentro do qual ocorre o equilíbrio térmico entre íons e elétrons [39,45]. Observe que existe uma hierearquia entre as frequências de colisão, $\nu_{\text {ie }} \ll \nu_{\text {ii }} \ll \nu_{\text {ei }}$.

\subsection{Campo magnético e equilíbrio no tokamak}

No tokamak, o campo magnético de equilíbrio pode ser representado por [46]

$$
\mathbf{B}=F \nabla \phi+\nabla \phi \times \nabla \Psi
$$

onde $F$ e $\Psi$ são funções relacionadas à componente toroidal $(\phi)$ do campo magnético e ao fluxo magnético poloidal $(\theta)$, respectivamente. Note que a condição $\boldsymbol{\nabla} \cdot \mathbf{B}=0$ é automaticamente satisfeita por (2.11).

Quando não há rotação de equilíbrio $(\mathbf{V}=0), F=F(\Psi), p=p(\Psi)$ e a condição de estabilidade do plasma pode ser escrita como

$$
\mathbf{J} \times \mathbf{B}=\nabla p
$$

onde $\mathbf{J}=\mu_{0}^{-1} \nabla \times \mathbf{B}$ é a densidade de corrente no plasma. De forma equivalente, (2.12) pode ser representada pela equação de Grad-Shafranov [47-49],

$$
\Delta^{*} \Psi+\mu_{0} R^{2} \frac{d p}{d \Psi}+\frac{1}{2} \frac{d F}{d \Psi}=0, \quad \Delta^{*} \Psi=R^{2} \nabla \cdot\left(\frac{\nabla \Psi}{R^{2}}\right)
$$

na qual $\Delta^{*}$ é um operador elíptico conhecido como operador de Shafranov [46].

A razão entre o raio maior e o raio menor do tokamak é importante parâmetro conhecido 
como razão de aspecto,

$$
A=\frac{R_{0}}{a} \leq \frac{1}{\varepsilon}, \quad \varepsilon=\frac{r}{R_{0}}
$$

Em tokamaks de seç̧ão circular e alta razão de aspecto $(\varepsilon \ll 1)$, a dependência com a posição poloidal $(\theta)$ de $\Psi$ pode ser determinada analiticamente através de (2.13), mas não a dependência radial $[41,46]$. De forma aproximada, obtém-se que $\Psi(r, \theta) \approx \Psi_{0}(r)\left[1+\left(\Delta_{s}(r) / R_{0}\right) \cos \theta\right]$, onde $\Delta_{s}(r)$, ou deslocamento de Shafranov, é uma medida do quanto as superfícies magnéticas se deslocam em relação ao centro da coluna de plasma.

Na presença de rotação de equilíbrio [41], caso que consideramos no capítulo 3, o termo $\rho(\mathbf{V} \cdot \boldsymbol{\nabla}) \mathbf{V}$ deve ser adicionado ao lado direito de (2.12), o que resulta na equação de GradShafranov modificada [50,51],

$$
\begin{aligned}
& \left(1-\frac{\mu_{0} \kappa^{2}}{\rho}\right) \Delta^{*} \Psi+\mu_{0} R^{2}\left(\frac{d p}{d \Psi}\right)_{R}+\frac{1}{2}\left(\frac{d F^{2}}{d \Psi}\right)_{R}-\mu_{0} \kappa \nabla \Psi \cdot \nabla\left(\frac{\kappa}{\rho}\right)+ \\
& \frac{\mu_{0} \rho}{2} \frac{d}{d \Psi}\left(\frac{\kappa^{2}}{\rho^{2}}|\nabla \Psi|^{2}\right)_{R}=0
\end{aligned}
$$

onde $\kappa=\kappa(\Psi)$ é uma função de fluxo proporcional à velocidade de rotação poloidal e o índice $R$ indica que as derivadas com relação a $\Psi$ devem ser calculadas a $R$ constante. Se não levarmos em conta injeção ou perda de partículas do plasma, a velocidade de equilíbrio, de acordo com as equações da continuidade e da lei de Ohm ideal, pode ser expressa como

$$
\mathbf{V}=\frac{\kappa}{\rho} \mathbf{B}-\frac{d \Phi}{d \Psi} R^{2} \nabla \phi
$$

onde $\Phi=\Phi(\Psi)$ é o potencial eletrostático de equilíbrio.

A relação entre a componente poloidal e a toroidal do campo magnético é descrita por um parâmetro amplamente presente em muitos modos importantes do tokamak, o fator de segurança, que pode ser definido como [44,46, 50]:

$$
q=q(\Psi)=\frac{\mathbf{B} \cdot \boldsymbol{\nabla} \phi}{\mathbf{B} \cdot \boldsymbol{\nabla} \theta}=\int d \theta \frac{d \phi}{d \theta}
$$


Este parâmetro é uma medida da helicidade das linhas de força no plasma e está diretamente ligado à estabilidade do plasma, que requer que o limite de Kruskal-Shafranov (KS) [52,53], $q>1$, seja satisfeito pelo menos no centro da coluna de plasma. Em tokamaks, o fator de segurança costuma ser maior na borda ( $q \sim 3$ ou até mesmo $q \sim 5)$ do que no centro $(q \sim 1)$. Uma outra grandeza importante para a estabilidade, diretamente relacionada a este parâmetro, é o cisalhamento magnético,

$$
s(r)=\frac{r}{q} \frac{d q}{d r}
$$

que adimensionalmente expressa a variação de $q$ com a posição radial. A medida desta grandeza tem importância fundamental na área de diagnósticos para a determinação do perfil radial de $q$.

No estudo analítico de modos de baixas frequências, assim como em modelos neoclássicos, é possível em muitos casos utilizar a aproximação:

$$
\mathbf{B}=\frac{B}{1+\varepsilon \cos \theta}\left(\frac{\varepsilon}{q(r)} \hat{\mathbf{e}}_{\theta}+\hat{\mathbf{e}}_{\phi}\right)
$$

onde $\hat{\mathbf{e}}_{\theta}$ e $\hat{\mathbf{e}}_{\phi}$ são versores na direção poloidal e toroidal, respectivamente. Em modelos locais, como é o caso desta tese, em muitos casos podemos desconsiderar o efeito de cizalhamento magnético.

Note que o campo magnético mostrado em (2.19) é simétrico com relação a $\phi$ mas não com relação ao ângulo poloidal $(\theta)$, de forma que o campo magnético é ligeiramente maior na parte interna do tokamak $\left(\mathrm{HFS}^{5}\right)$ do que na parte externa $\left(\mathrm{LFS}^{6}\right)$. Apesar de pequena, esta diferença, $\Delta B / B \sim \varepsilon$, desempenha um impacto significativo nos valores dos coeficientes de transporte. No estudo de transporte, os coeficientes clássicos de transporte, provenientes da teoria de gases neutros e de plasmas magnetizados em sistemas cilíndricos, devem ser substituidos pelos coeficientes neoclássicos, que são muito maiores e, em muitos casos, podem até chegar a ser uma ordem de magnitude superior. Uma breve discussão sobre transporte em tokamaks é apresentada na

\footnotetext{
${ }^{5}$ High field side

${ }^{6}$ Low Field Side
} 
seção seguinte.

\subsection{Difusão e transporte em tokamaks}

Um dos desafios mais importantes da física de plasma confinados magneticamente é o de reduzir a perda de partículas e energia em tokamaks. Com esta finalidade foram desenvolvidas teorias de transporte, que consistem essencialmente em determinar os coeficientes $D$ e $\kappa$ referentes aos fluxos de partículas e de calor, respectivamente, os quais dependem de gradientes de densidade e de temperatura [44], ou seja,

$$
\boldsymbol{\Gamma} \approx-D \boldsymbol{\nabla}_{\perp} n \quad \text { e } \quad \mathbf{q} \approx-\kappa \boldsymbol{\nabla}_{\perp} T
$$

Em (2.20), $D$ é o coeficiente de difusão e $\kappa$ é a condutividade térmica. As leis de convservação de partículas e energia podem ser enunciadas como

$$
\begin{aligned}
& \frac{\partial n}{\partial t}+\nabla \cdot \boldsymbol{\Gamma}=S_{\text {part }}, \\
& \frac{3}{2} \frac{\partial T}{\partial t}+\boldsymbol{\nabla} \cdot \mathbf{q}=S_{\mathrm{cal}},
\end{aligned}
$$

onde $S_{\text {part }}$ e $S_{\text {cal }}$ representam fontes externas de partículas e de calor. Os fluxos $\boldsymbol{\Gamma}$ e q são quantidades macroscópicas do plasma que, experimentalmente, são medidos por meio de diagnósticos e, teoricamente, podem ser estimados. Para o coeficiente de difusão, por exemplo, através de análise dimensional, podemos estimar $D$ :

$$
D \sim \nu(\Delta r)^{2}
$$

onde $\nu$ é a frequência de colisões e $\Delta r$ é o comprimento característico.

A teoria de transporte clássico, a qual se aplica a sistemas de geometrica cilíndrica, é discutida em detalhes por R. Balescu [54]. Já no tokamak, devido ao efeito da geometria deste no campo magnético de equilíbrio, não há simetria poloidal, fato este ao qual nos referimos como efeito neoclássico e, desta forma, a teoria de transporte neoclássico [55,56] deve ser aplicada 
neste caso. No caso cilíndrico o raio de Larmor $(\rho)$ representa o comprimento característico para a quantificação da difusão perpendicular, ou seja,

$$
D_{\perp} \sim \nu \rho^{2}, \quad \kappa_{\perp} \sim n \nu \rho^{2} .
$$

Em contrapartida, devido a liberdade de locomoção das partículas ao longo do campo magnético, limitada apenas por colisões, é possível estimar o coeficiente de condutividade de calor paralelo como,

$$
\kappa_{\|} \sim n \nu \lambda^{2} \sim \frac{\omega_{c}^{2}}{\nu^{2}} \kappa_{\perp}
$$

onde $\lambda=v_{T_{\alpha}} / \nu$ é o livre caminho médio. Como $\omega_{c} / \nu \gg 1$ para plasmas magnetizados, é possível concluir que o calor se difunde muito mais facilmente ao longo do campo magnético e, quando o plasma se torna mais colisional, a condutividade paralela diminui enquanto que a condutividade perpendicular aumenta, fazendo com que o plasma tenda a perder mais energia.

Os parágrafos anteriores não levam em conta a assimetria poloidal do campo magnético, o que o torna mais intenso no lado interno da coluna de plasma $\left(\mathrm{HFS}^{7}\right)$ do que no lado externo $\left(\mathrm{LFS}^{8}\right)$. Essa assimetria tem influência no movimento do centro guia das partículas, conforme descrito qualitativamente em [6] e quantitativamente em $[44,56]$. Em consequência algumas partículas, cuja velocidade paralela é relativamente baixa, não conseguem vencer a barreira do poço magnético, $\Delta B / B \sim \varepsilon$ e, em consequência retornam, porém, em outra superfície magnética. O resultado desse processo que se repete por alguns ciclos, é que essas partículas apresentam órbitas irregulares, conhecidas como órbitas de banana, com um deslocamento efetivo estimado por $\Delta r \sim(q / \sqrt{\varepsilon}) \rho \gg \rho$. Para o cálculo dos coeficientes de transporte é necessário levar em conta não somente o movimento das partículas afetando seu deslocamento característico, mas também sua frequência efetiva de colisões.

Essencialmente, há três regimes fundamentais a ser considerado:

- Pfirsch-Schlüter: Neste regime, também conhecido como regime colisional ou regime de

\footnotetext{
${ }^{7}$ High field side - lado de campo maior

${ }^{8}$ Low Field Side - lado de menor campo
} 
fluidos, o tempo necessário para que as partículas possam completar uma órbita é maior do que o tempo de colisão, de forma que as órbitas das partículas são constantemente interrompidas por colisões. Desta forma, este regime é descrito pela condição: $\nu / \omega_{\operatorname{tr}} \gg 1$, onde $\omega_{\mathrm{tr}}=v_{T} / q R_{0}$ é a frequência de circulação. Este regime descreve bem a borda da coluna de plasma e o coeficiente de difusão perpendicular pode ser estimado como [44,57]:

$$
D_{\perp}^{(P S)} \sim q^{2} \nu \rho^{2}
$$

ou seja, da ordem de $q^{2}$ maior do que o esperado pela teoria clássica.

- Plateau: Neste regime, $\varepsilon^{3 / 2} \ll \nu / \omega_{\text {tr }} \ll 1$ e as equações de fluido não se aplicam, sendo necessário o uso da equação cinética de deriva. Trata-se de uma condição intermediária entre o regime Pfirsch-Schlüter e o regime de banana que se aplica ao centro da coluna de plasma e tem, como coeficiente de difusão, a expressão [44]:

$$
D_{\perp}^{(P)} \sim \frac{\omega_{\text {tr }}}{\nu} q^{2} \nu \rho^{2}
$$

ou seja, tal coeficiente é bem maior do que no caso anterior, pois $\omega_{\operatorname{tr}} / \nu \gg 1$.

- Banana: É o regime não colisional - as partículas, em média, possuem tempo suficiente para completar suas órbitas antes de colidirem com outras. Entretanto, devido ao grande comprimento característico destas órbitas, a colisão ocorre fora de suas superfícies magnéticas de origem, o que acarreta uma grande contribuição para o transporte radial. A frequência de colisão é descrita por $\nu / \omega_{\text {tr }} \ll \varepsilon^{3 / 2}$ e a estimativa para o coeficiente de difusão resulta em [44]

$$
D_{\perp}^{(B)}=\frac{q^{2}}{\varepsilon^{3 / 2}} \nu \rho^{2},
$$

e, portanto, o fator multiplicativo $q^{2} / \varepsilon^{3 / 2}$ em relação ao valor clássico para o coeficiente de difusão faz com este possa ser de até uma ordem de magnitude maior. O modelo a ser adotado também se baseia na equação cinética de deriva de forma que o modelo de fluido 
também não se aplica neste regime.

Importantes trabalhos publicados sobre transporte neoclássico $[56,58,59]$ e, até mesmo, livros que tratam o assunto com bastante riqueza de detalhes [30,55] têm a finalidade de fornecer uma boa compreensão sobre efeitos neoclássicos e seus impactos no confinamento de plasma. Entretanto, em regiões dominadas por processos turbulentos, o coeficiente de difusão é ainda maior do que os descritos pelos modelos neoclássicos. Neste caso, ocorre o que chamamos de difusão de Bohm [60] e, na área de transporte turbulento (ou transporte anômalo), discussão abrangente sobre este tipo de transporte é feita por R. Balescu [40], como continuação de seus trabalhos iniciais $[54,55]$.

Embora não seja o foco desta tese tratar sobre transporte turbulento, uma simples estimativa, de acordo com [44], para o coeficiente de difusão anômala é útil a título de comparação com os coeficientes neoclássicos. Para elétrons, $D_{\perp e}=(\Delta r)^{2} / \tau$, podemos estimar $\Delta r / \tau \sim v_{E} \sim \Phi / \Delta r B$ e $e \Phi / T=k$, onde $v_{E}$ é a velocidade de deriva fundamental $(\boldsymbol{E} \times \mathbf{B})$. Segue, portanto, que

$$
D_{\perp e}^{(\mathrm{Bohm})}=k \frac{T}{e B} \sim \omega_{c} \rho^{2}
$$

Note que, como $\omega_{c} \gg q^{2} \nu / \varepsilon^{3 / 2}, D_{\perp e}^{(\mathrm{Bohm})} \gg D_{\perp e}^{(B)}$.

Historicamente tal coeficiente foi descrito como $D_{e}^{(\mathrm{Bohm})}=T / 16 e B$, onde a razão para o fator 1/16 até hoje permanece obscura [6]. Trata-se de um difícil problema não-linear a determinação de $k$, sendo que $k<1$ [44]. Para concluir esta seção, observamos que o valor do coeficiente de difusão anômala para elétrons excede o valor clássico em aproximadamente $k \omega_{c_{e}} \tau_{\mathrm{ei}} \gg 1$ e, por isso, impedir a degradação do confinamento devido ao transporte anômalo é considerado um dos maiores desafios da física de tokamaks. 


\subsection{Movimento de partículas e velocidade do centro guia}

Partículas carregadas imersas em um campo eletromagnético, como as que compôem o plasma, ficam submetidas à ação da força de Lorentz, de acordo com a equação a seguir, que exemplifica a dinâmica de íons:

$$
\frac{d \mathbf{v}}{d t}=\frac{e}{m}[\boldsymbol{E}(\mathbf{r}, t)+\mathbf{v} \times \mathbf{B}(\mathbf{r}, t)]
$$

onde, $\mathbf{v}=d \mathbf{r} / d t$ é a velocidade destas partículas que estão localizadas na posição $\mathbf{r}$. Note que nesta equação, bem como nas próximas desta seção, omitimos o índice $\alpha$, o qual deve ser subentendido.

A velocidade em (2.29) pode ser expressa na forma

$$
\mathbf{v}=v_{\|} \mathbf{b}+\mathbf{v}_{\perp}, \quad \mathbf{v}_{\perp}=v_{\perp}\left(\cos \gamma \hat{\mathbf{e}}_{1}-\sin \gamma \hat{\mathbf{e}}_{2}\right)
$$

onde $\gamma=-\tan ^{-1}\left(\mathbf{v} \cdot \hat{\mathbf{e}}_{1} / \mathbf{v} \cdot \hat{\mathbf{e}}_{2}\right)$ é o $\hat{a}$ ngulo de giração e (b, $\left.\hat{\mathbf{e}}_{1}, \hat{\mathbf{e}}_{2}\right)$ formam, nesta ordem, uma base ortonormal convencionalmente orientada, na qual $\mathbf{b}=\mathbf{B} / B$.

De forma similar, a posição das partículas em um plasma magnetizado pode ser expressa como:

$$
\mathbf{r}=\mathbf{r}_{g}+\boldsymbol{\rho}, \quad \boldsymbol{\rho}=\frac{\mathbf{b} \times \mathbf{v}_{\perp}}{\omega_{c}}
$$

onde $\boldsymbol{\rho}$ é o raio de Larmor vetorial e $\mathbf{r}_{g}$ é a posição do centro guia, o ponto central da órbita aproximadamente circular das partículas. O nosso principal interesse nesta seção está na obtenção da velocidade do centro guia, a qual é obtida derivando com relação ao tempo (2.31) e, em seguida, tomando a média em relação ao ângulo de giração. Para a média de uma grandeza genérica, $X$, atribuimos a definição:

$$
\langle X\rangle=\frac{1}{2 \pi} \int_{0}^{2 \pi} d \gamma X
$$


Ao observar que $\langle\mathbf{v}\rangle=v_{\|} \mathbf{b}$ e $\langle\boldsymbol{\rho}\rangle=0$, obtém-se

$$
\mathbf{v}_{g}=\left\langle\frac{d \mathbf{r}_{g}}{d t}\right\rangle=v_{\|} \mathbf{b}-\left\langle\frac{d \boldsymbol{\rho}}{d t}\right\rangle
$$

onde o cálculo de $\langle d \boldsymbol{\rho} / d t\rangle$, que é relativamente longo, pode ser visto em $[30,56,61,62]$.

Antes de apresentarmos a velocidade do centro guia, é conveniente definir o momento magnético e a energia de uma partícula,

$$
\mu_{\alpha}=\frac{m_{\alpha} v_{\perp}^{2}}{2 B} \quad \text { e } \quad \mathcal{E}_{\alpha}=e_{\alpha} \Phi+\mu_{\alpha} B+\frac{m_{\alpha} v_{\|}^{2}}{2}
$$

que, em primeira ordem em $\rho / L$, onde $L$ representa genéricamente o comprimento característico do gradiente de qualquer quantidade macroscópica do plasma, são constantes de movimento, da mesma forma que o momento canônico paralelo [58,63].

Em primeira ordem em $\delta_{\rho}=\rho_{i} / L$, a velocidade do centro guia pode ser expressa como

$$
\mathbf{v}_{g_{\alpha}}=v_{\| \alpha} \mathbf{b}+\mathbf{v}_{\mathrm{E}}+\mathbf{v}_{B \alpha}+\mathbf{v}_{\boldsymbol{\kappa} \alpha},
$$

onde

$$
\mathbf{v}_{\mathrm{E}}=\frac{\boldsymbol{E} \times \mathbf{B}}{B^{2}},
$$

é a deriva $\boldsymbol{E} \times \boldsymbol{B}$, que pode ser de ordem $\delta_{\rho}^{0}$ (MHD), assim como $v_{\|}$, ou de ordem $\delta_{\rho}^{1}$ (drift), conforme discutido na próxima seção. Esta deriva possui o mesmo sentido para íons e elétrons, pois independe da carga da partícula. A deriva magnética,

$$
\mathbf{v}_{B \alpha}=\frac{\mu_{\alpha}}{e_{\alpha}} \mathbf{b} \times \nabla \ln B
$$

surge devido à inomogeneidades do campo magnético e, por estar relacionada ao movimento ciclotrônico, possui sentidos opostos para cargas positiva e negativa e, finalmente,

$$
\mathbf{v}_{\boldsymbol{\kappa}}=\frac{e_{\alpha}}{\left|e_{\alpha}\right|} \frac{v_{\|}^{2}}{\omega_{c_{\alpha}}} \mathbf{b} \times \boldsymbol{\kappa},
$$


que também tem seu sentido de movimento dependente da carga da partícula, é a deriva resultande da curvatura do campo magnético, $\boldsymbol{\kappa}=(\mathbf{b} \cdot \nabla) \mathbf{b}$.

No geral, as velocidades de deriva podem ser expressas na forma

$$
\mathbf{v}_{\mathrm{d} \alpha}=\frac{1}{m_{\alpha}} \frac{\mathbf{F}_{\alpha} \times \mathbf{B}}{B^{2}}
$$

onde $\mathbf{F}_{\alpha}$ representa as diversas forças que agem na partícula, ou seja, elétrica, magnética de curvatura, etc...

\subsection{Teoria cinética}

Um dos objetivos da teoria cinética é determinar a função distribuição $\left(f_{\alpha}\right)$ para cada espécie de partícula, pois a partir do cálculo de momentos desta função, isto é, da integral com relação às coordenadas da velocidade da função distribuição multiplicada por potências da velocidade, obtém-se grandezas macroscópicas do plasma, as quais podem ser comparadas com valores experimentais. As equações de fluido, obtidas a partir desta metodologia, conforme discutido em 2.6.1, descrevem importantes leis físicas no que se refere a conservação de mensuráveis macroscópicos do plasma.

Em princípio considera-se que a função distribuição é da forma, $f_{\alpha}=f_{\alpha}(t, \mathbf{r}, \mathbf{v})$, porém, no modelo girocinético, utilizado no capítulo 4 , a forma $f_{\alpha}^{(g)}=f_{\alpha}^{(g)}\left(t, \mathbf{r}_{g}, \mu, \mathcal{E}, \gamma\right)$ é mais conveniente. A última forma é utilizada na derivação da equação girocinética, mostrada na seção 2.5.2. Entretanto, para estimar ordens de grandeza de termos da equação de Boltzmann, utilizamos a primeira forma.

\subsubsection{Análise da equação de Boltzmann}

De uma forma geral, as equações cinéticas podem ser expressas como

$$
\frac{d f}{d t}=\sum_{i=0}^{6} \frac{d x_{i}}{d t} \frac{\partial f}{\partial x_{i}}=C(f)
$$


onde $f=f\left(x_{0}, x_{1}, x_{2}, x_{3}, x_{4}, x_{5}, x_{6}\right)$ é a função distribuição de partículas no espaço de fase descrito pelas variáveis $x_{i}, x_{0}=t$ e $C(f)$ é o termo de colisões. A equação de Boltzmann, cuja obtenção a partir de leis mais gerais e sua interpretação física são apresentadas de forma clara e abrangente em [31], é o caso particular em que $x_{1}, x_{2}, x_{3}$ são as coordenadas espaciais da localização instantânea da partícula e $x_{4}, x_{5}, x_{6}$ são as componentes da velocidade desta. Esta equação, que normalmente é utilizada na obtenção do tensor dielétrico [35], é expressa como

$$
\frac{\partial f_{\alpha}}{\partial t}+\mathbf{v} \cdot \frac{\partial f_{\alpha}}{\partial \mathbf{r}}+\mathbf{a}_{\alpha} \cdot \frac{\partial f_{\alpha}}{\partial \mathbf{v}}=C_{\alpha}(f),
$$

onde $\mathbf{a}_{\alpha}$ é a aceleração da partícula to tipo $\alpha$ com velocidade $\mathbf{v}$ localizada em $\mathbf{r}$ que, para plasmas de laboratório, é bem descrita pela expressão

$$
\mathbf{a}_{\alpha}=\frac{e_{\alpha}}{m_{\alpha}}(\boldsymbol{E}+\mathbf{v} \times \mathbf{B}) .
$$

No contexto da física de tokamaks, o plasma é considerado magnetizado quando $\rho_{i} / L_{B} \ll 1$, onde $L_{B}$ é o comprimento característico referente ao gradiente do campo magnético de equilíbrio no plasma. Além disso, consideramos, nesta tese, fenômenos de baixas frequências quando comparadas com a frequência de cíclotron e admite-se, na análise desta seção, que o comprimento de onda perpendicular $\left(\lambda_{\perp}\right)$ das perturbações possam ser da ordem do raio de Larmor, característico de violentas instabilidades [30].

As informações do parágrafo anterior podem ser expressas da seguinte forma:

$$
\delta_{\rho}=\frac{\rho_{i}}{L} \ll 1, \quad \delta_{k}=k_{\perp} \rho_{e} \ll 1, \quad \delta_{\omega}=\frac{\omega_{t}}{\omega_{c_{i}}} \ll 1
$$

onde $L$ é o maior dos comprimentos característicos referentes aos gradientes de quantidades macroscópicas de equilíbrio e $\omega_{\mathrm{tr}} \leq v_{T_{e}} / L$ é a frequência de circulação relacionada a variação temporal da função distribuição. Consideramos, ainda, que a frequência de colisão é pequena se comparada com a frequência ciclotrônica, $\nu / \omega_{c} \sim \delta_{\rho} \ll 1$ e, de fato, para simplificar, na derivação da equação girocinética não levamos em conta o operador de colisão. 
Antes de discutir sobre a equação cinética de deriva ${ }^{9}$ e a equação girocinética $^{10}$, as quais são largamente utilizada no estudo de modos de baixas frequências [64-66], é conveniente estimar a ordem de grandeza dos termos da equação de Boltzmann, conforme o fazemos a seguir:

$$
\begin{gathered}
\frac{\partial f}{\partial t} \sim \delta_{\rho} \omega_{c} f, \quad \frac{e}{m}(\mathbf{v} \times \mathbf{B}) \cdot \frac{\partial f}{\partial \mathbf{v}} \sim \omega_{c} f, \quad C(f) \sim \nu f \sim \delta_{\rho} \omega_{c}, \\
\frac{e}{m} E_{\|} \mathbf{b} \cdot \frac{\partial f}{\partial \mathbf{v}} \sim \frac{E_{\|} / B}{v_{T}} \omega_{c} f \sim \delta_{\rho} \omega_{c} f \quad \text { e } \quad \frac{e}{m} \mathbf{E}_{\perp} \cdot \frac{\partial f}{\partial \mathbf{v}} \sim \frac{v_{E}}{v_{T}} \omega_{c} f .
\end{gathered}
$$

Em (2.45), $v_{E}=|\boldsymbol{E} \times \mathbf{B}| / B^{2}$ e $E_{\|} / B \sim \delta_{\rho} v_{T_{i}}$ próximo do equilíbrio [30].

Quanto à análise do termo convectivo, $\mathbf{v} \cdot \nabla f$, considera-se que a função distribuição é composta por duas partes, $f \sim F+\tilde{G}$. Enquanto que a primeira varia suavemente no espaço $(|\nabla F| \sim F / L)$, a segunda consiste de perturbações de pequenos comprimentos de onda $(|\nabla \tilde{G}| \sim \tilde{G} / \lambda)$, onde a condição $\lambda \sim \rho_{i}$ é possível. Normalmente as equações cinéticas são resolvidas de forma perturbativa, considerando que $\tilde{G} \sim \delta_{k} F$, de forma que

$$
\mathbf{v} \cdot \nabla f \sim \omega F+\omega_{c} \tilde{G} \sim\left(\delta_{\rho}+\delta_{k}\right) \omega_{c} f
$$

e, consequentemente há dois parâmetros independentes a ser analisado, $\delta_{\rho} \ll 1$ e $\delta_{k} \ll 1$. Tal análise inclui três casos de bastante interesse para tokamaks:

- Ordem de deriva: Considera-se $\delta_{k}=0$ e $v_{E} \sim \delta_{\rho} v_{T_{i}}$, de forma que $\mathbf{v} \approx v_{\|} \mathbf{b}$, e normalmente utiliza-se a equação cinética de deriva como ponto de partida para modelos. Este tipo de ordem é largamente aplicado para descrever processos de transporte diversos tipos de instabilidades.

- Ordem MHD: Neste caso $\delta_{k}=0$ mas $v_{E} \sim v_{T_{i}}$, ou seja, $\mathbf{v} \approx v_{\|} \mathbf{b}+\mathbf{v}_{E}$. A teoria de fluidos (veja a seção 2.6), que é bastante clara do ponto de vista físico, pode ser utilizada em fenômenos que envolvam este tipo de ordem, tais como violentas instabilidades MHD.

\footnotetext{
${ }^{9}$ drift kinetic equation

${ }^{10}$ gyrokinetic equation
} 
- Ordem de giração: É o caso em que, embora $v_{E} \sim \delta_{\rho} v_{T_{i}}$ admite-se a possibilidade de perturbações com grandes variações espaciais $\left(\delta_{k} \sim \delta_{\rho}\right)$. A equação girocinética deve ser utilizada neste caso.

\subsubsection{A equação girocinética}

A seguir, baseados nas refs. [30,32], apresentamos os principais passos para a obtenção da equação girocinética, a qual é utilizada no capítulo 4.

É conveniente considerar a mudança de variáveis $(t, \mathbf{r}, \mathbf{v}) \rightarrow\left(t, \mathbf{r}_{g}, \mu, \mathcal{E}_{\alpha}, \gamma\right)$ em relação às variáveis da equação de Boltzmann, de forma que a eq. (2.41) possa ser escrito como

$$
\frac{\partial f}{\partial t}+\frac{d \mathbf{r}_{g}}{d t} \cdot \frac{\partial f}{\partial \mathbf{r}_{g}}+\frac{d \mu}{d t} \frac{\partial f}{\partial \mu}+\frac{d \mathcal{E}_{\alpha}}{d t} \frac{\partial f}{\partial \mathcal{E}_{\alpha}}+\frac{d \gamma}{d t} \frac{\partial f}{\partial \gamma}=0
$$

O procedimento para obtenção da equação girocinética consiste em calcular a média de (2.47) com relação a $\gamma$ considerando, para este cálculo, que $\mathbf{r}_{g}=\mathbf{r}_{g}(\mathbf{r}, \mathbf{v}), \mu=\mu\left(\mathbf{r}_{g}, \mathbf{v}\right), \mathcal{E}_{\alpha}=\mathcal{E}_{\alpha}\left(\mathbf{r}_{g}, \mathbf{v}\right)$ e $\gamma=\gamma\left(\mathbf{r}_{g}, \mathbf{v}\right)$.

Para o densenvolvimento analítico nas próximas etapas, adota-se a aproximação eikonal com relação à coordenada do centro guia,

$$
\mathbf{X}(\mathbf{r})=\mathbf{X}_{g}\left(\mathbf{r}_{g}\right) \mathrm{e}^{i \mathbf{k}_{\perp} \cdot \mathbf{r}},
$$

onde $\mathbf{X}$ representa de forma genérica qualquer vetor. Ao tomar a média de (2.48) obtemos

$$
\langle\mathbf{X}(\mathbf{r})\rangle=J_{0}\left(k_{\perp} \rho\right) \mathbf{X}_{g}\left(\mathbf{r}_{g}\right) \mathrm{e}^{i \mathbf{k}_{\perp} \cdot \mathbf{r}_{g}},
$$

onde $J_{n}(x)$ é a função de Bessel de ordem $n$ e de argumento $x$ e, em (2.49), foi utilizada a relação $\left\langle\mathrm{e}^{i \mathbf{k}_{\perp} \cdot \boldsymbol{\rho}}\right\rangle=J_{0}\left(k_{\perp} \rho\right)[30,32]$. A partir de (2.31), (2.33) e (2.34) obtém-se [30]:

$$
\begin{aligned}
& \left\langle\frac{d \mathbf{r}_{g}}{d t}\right\rangle=\mathbf{v}_{g}+\left[J_{0}\left(k_{\perp} \rho\right)\left(\tilde{\Phi}-v_{\|} \tilde{A}_{\|}\right)+2 \frac{J_{1}\left(k_{\perp} \rho\right)}{k_{\perp} \rho} \frac{\mu}{e} \tilde{B}_{\|}\right] \frac{i \mathbf{k}_{\perp} \times \mathbf{b}}{B}, \quad\left\langle\frac{d \mu}{d t}\right\rangle \approx 0, \\
& \left\langle\frac{d \mathcal{E}}{d t}\right\rangle=\frac{\partial}{\partial t}\left[J_{0}\left(k_{\perp} \rho\right) e\left(\tilde{\Phi}-v_{\|} \tilde{A}_{\|}\right)+2 \frac{J_{1}\left(k_{\perp} \rho\right)}{k_{\perp} \rho} \mu \tilde{B}_{\|}\right],
\end{aligned}
$$


onde a notação $\tilde{X}=\tilde{X}\left(\mathbf{r}_{g}, t\right) \operatorname{com} \tilde{X}=\tilde{\Phi}, \tilde{A}_{\|}, \tilde{B}_{\|}$identifica as perturbações.

A função distribuição pode ser decomposta em três partes,

$$
f_{\alpha}=F_{\alpha}+\tilde{\mathcal{G}}_{\alpha}+\tilde{\mathcal{G}}_{\alpha}^{(\gamma)}
$$

onde $F_{\alpha}$ é a contribuição de equilíbrio, $\tilde{\mathcal{G}}_{\alpha}+\tilde{\mathcal{G}}_{\alpha}^{(\gamma)}=\left(\mathcal{O}\left(\delta_{\rho}\right)+\mathcal{O}\left(\delta_{k}\right)\right) F_{\alpha}$ é a perturbação e $\tilde{G}_{\alpha}^{(\gamma)}$ é a parte dependente de $\gamma$. Em primeira ordem em $\delta_{\rho}$ e $\delta_{k}$, o cálculo da média de (2.47) resulta na equação girocinética,

$$
\begin{aligned}
& \left(\frac{\partial}{\partial t}+\mathbf{v}_{g_{\alpha}} \cdot \nabla\right) \tilde{g}_{\alpha}= \\
& e_{\alpha}\left(\frac{\partial F_{\alpha}}{\partial \mathcal{E}_{\alpha}} \frac{\partial}{\partial t}+\frac{\mathbf{b} \times \boldsymbol{\nabla} F_{\alpha}}{m_{\alpha} \omega_{c_{\alpha}}} \cdot i \mathbf{k}_{\perp}\right)\left[J_{0}\left(k_{\perp} \rho_{\alpha}\right)\left(\tilde{\Phi}-v_{\|} \tilde{A}_{\|}\right)+2 \frac{J_{1}\left(k_{\perp} \rho_{\alpha}\right)}{k_{\perp} \rho_{\alpha}} \frac{\mu_{\alpha}}{e_{\alpha}} \tilde{B}_{\|}\right] .
\end{aligned}
$$

onde os gradientes são avaliados nas coordenadas do centro guia $\left(\mathbf{r}_{g}\right)$. Em tokamaks, a aproximação $\tilde{B}_{\|} \approx 0$ pode ser considerada na maiorida dos modelos [67] e, de fato, é considerada no capítulo 4 .

É importante observar que $\tilde{g}_{\alpha}$ não representa integralmente a parte perturbada da função distribuição, a qual é obtida pela expansão de $f_{\alpha}$ em torno da energia de equilíbrio, $\mathcal{E}_{\alpha 0}=$ $e_{\alpha} \Phi+m_{\alpha} v^{2} / 2$, onde $\Phi$ é o potencial eletrostático de equilíbrio ${ }^{11}, \mathcal{E}_{\alpha}=\mathcal{E}_{\alpha 0}+\mathcal{E}_{\alpha 1}$ é a energia total e $\mathcal{E}_{\alpha 1}=e_{\alpha} \tilde{\Phi}$ é a perturbação da energia. Conforme a equação (2.51), segue que

$$
f_{\alpha} \approx F_{\alpha}\left(\mathcal{E}_{\alpha 0}\right)+\left.\mathcal{E}_{\alpha 1} \frac{\partial F_{M_{\alpha}}}{\partial \mathcal{E}_{\alpha}}\right|_{\mathcal{E}_{\alpha}=\mathcal{E}_{\alpha 0}}+\tilde{G}_{\alpha}^{(\gamma)}
$$

onde $\tilde{G}_{\alpha}^{(\gamma)}$ é a contribuição dependente de $\gamma$ e proveniente da equação girocinética, (2.52), ou seja,

$$
f_{\alpha}=F_{\alpha}+\tilde{f}_{\alpha}, \quad \tilde{f}_{\alpha}=e_{\alpha} \tilde{\Phi} \frac{\partial F_{M_{\alpha}}}{\partial \mathcal{E}_{\alpha}}+\tilde{g}_{\alpha} \mathrm{e}^{i \mathbf{k}_{\perp} \cdot \boldsymbol{\rho}_{\alpha}}
$$

No capítulo 4 , utilizamos a substituição $\boldsymbol{\nabla} \rightarrow i\left(\mathbf{k}_{\perp}+\mathbf{b} \hat{k}_{\|}\right)$para resolver (2.52), onde

$$
\mathbf{k}_{\perp} \approx \hat{\mathbf{e}}_{r} \mathrm{k}_{r}+\hat{\mathbf{e}}_{\theta} \frac{1}{r} \frac{\partial}{\partial \theta}, \quad \hat{k}_{\|}=\frac{1}{q R_{0}}\left(\frac{\partial}{\partial \theta}+q \frac{\partial}{\partial \phi}\right) .
$$

\footnotetext{
${ }^{11}$ Conforme mostrado no capítulo 3 , este potencial existe se, e somente se, há rotação de equilíbrio.
} 


\subsubsection{Equação cinética de deriva}

Trata-se de uma equação que aparece principalmente em estudos sobre transporte neoclássico mas, também, pode ser utilizada na investigação de modos acústicos geodésicos (GAM), como em [68], por exemplo. Esta equação, que pode ser obtida de forma recursiva $[30,56,69]$ através do processo de giro-média, pode ser escrita como

$$
\frac{\partial f_{\alpha}}{\partial t}+\mathbf{v}_{g_{\alpha}} \cdot \nabla f_{\alpha}+\left(e_{\alpha} \mathbf{v}_{g_{\alpha}} \cdot \tilde{\boldsymbol{E}}+\mu_{\alpha} \frac{\partial B}{\partial t}\right) \frac{\partial f_{\alpha}}{\partial \mathcal{K}_{\alpha}}=0
$$

onde $\mathcal{K}_{\alpha}=\mathcal{E}_{\alpha}-e_{\alpha} \Phi$ é a energia cinética de partículas do tipo $\alpha$ e $\tilde{\boldsymbol{E}} \approx-\boldsymbol{\nabla} \tilde{\Phi}-\left(\partial \tilde{A}_{\|} / \partial t\right) \mathbf{b}$ é o campo elétrico perturbado. Uma derivação mais didática desta equação, baseada no trabalho original de R. D. Hazeltine [69], pode ser encontrada em [62].

\subsection{Teoria de fluidos}

A teoria de fluidos e a metodologia para obtenção de suas equações a partir da equação de Boltzmann são apresentados nesta seção. Apresentamos a teoria de dois fluidos e a teoria da magneto-hidrodinâmica (MHD), ambas utilizadas no capítulo 3.

\subsubsection{Teoria de dois fluidos e as equações de Braginskii}

O cálculo de momentos da equação de Boltzmann, (2.41), isto é, da integral de tal equação multiplicada por potências de combinações vetoriais da velocidade no espaço de velocidades, permite a obtenção das equações de fluidos ou equações de Braginskii. As equações de fluidos descrevem a evolução temporal de importantes quantidades macroscópicas do plasma que são discutidas a seguir.

Primeiramente, o cálculo do momento de ordem nula da função distribuição resulta na densidade de partículas,

$$
n_{\alpha}=n_{\alpha}(\mathbf{r}, t)=\int_{\mathbf{v}} f_{\alpha}(\mathbf{r}, \mathbf{v}, t) d^{3} \mathbf{v}
$$


a qual, quando combinada com as cargas dos diferentes tipos de partículas, permite a obtenção da densidade de carga,

$$
\rho_{c}=\sum_{\alpha} e_{\alpha} n_{\alpha}
$$

O momento de primeira ordem fornece o fluxo de partículas, $n_{\alpha} \mathbf{v}_{\alpha}$, o qual permite definir a velocidade do fluido do tipo $\alpha$,

$$
\mathbf{v}_{\alpha}=\frac{1}{n_{\alpha}} \int_{\mathbf{v}} \mathbf{v} f_{\alpha}(\mathbf{r}, \mathbf{v}, t) d^{3} \mathbf{v}
$$

e, de forma similar à densidade de carga, a densidade de corrente é obtida:

$$
\mathbf{j}=\sum_{\alpha} e_{\alpha} n_{\alpha} \mathbf{v}_{\alpha}
$$

Quando calculado no referencial do fluido, o segundo momento, fornece o tensor de pressãa, que pode ser decomposto na pressãa escalar cinética $\left(p_{\alpha}\right)$ e no tensor de viscosidade $(\boldsymbol{\pi})$, conforme mostrado abaixo:

$$
\mathbf{p}_{\alpha}=p_{\alpha} \mathbf{I}+\boldsymbol{\pi}_{\alpha}=\int_{\mathbf{v}} m_{\alpha}\left(\mathbf{v}-\mathbf{v}_{\alpha}\right)\left(\mathbf{v}-\mathbf{v}_{\alpha}\right) f_{\alpha}(\mathbf{r}, \mathbf{v}, t) d^{3} \mathbf{v}
$$

O tensor de viscosidade é normalmente dividido em três partes,

$$
\pi_{\alpha}=\pi_{\| \alpha}+\pi_{\mathrm{g} \alpha}+\pi_{\perp \alpha}
$$

denominadas viscosidade paralela, giro-viscosidade e viscosidade perpendicular. Finalmente, também calculado no referencial do fluido, o próximo e último momento de $f_{\alpha}$ que consideramos nesta tese fornece o fluxo de calor,

$$
\mathbf{q}_{\alpha}=\int_{\mathbf{v}} \frac{1}{2} m_{\alpha}\left[\left(\mathbf{v}-\mathbf{v}_{\alpha}\right) \cdot\left(\mathbf{v}-\mathbf{v}_{\alpha}\right)\right]\left(\mathbf{v}-\mathbf{v}_{\alpha}\right) f_{\alpha}(\mathbf{r}, \mathbf{v}, t) d^{3} \mathbf{v}
$$

Momentos da função distribuição de ordens mais alta não tem tanta importância do ponto de 
vista físico, apenas algébrico e, por esta razão e devido a sua pouca utilização nos modelos mais importantes, não os mostramos nesta tese. A seguir, apresentamos as quantidades dissipativas, que são calculadas a partir de momentos do operador de colisões, $C_{\alpha}(f)$. As duas quantidades de maior interesse para tokamaks são a força de fricção e o termo de transferência de calor, definidos, respectivamente por:

$$
\begin{gathered}
\mathbf{R}_{\alpha}=\int_{\mathbf{v}} m_{\alpha} \mathbf{v}_{\alpha} C_{\alpha}(f) d^{3} \mathbf{v} \\
Q_{\alpha}=\int_{\mathbf{v}} \frac{1}{2} m_{\alpha}\left(\mathbf{v}-\mathbf{v}_{\alpha}\right) \cdot\left(\mathbf{v}-\mathbf{v}_{\alpha}\right) C_{\alpha}(f) d^{3} \mathbf{v} .
\end{gathered}
$$

\section{As equações de Braginskii (ou equações de dois fluidos)}

Trata-se das das equações obtidas a partir do cálculo de momentos da equação de Boltzmann, (2.41), e descrevem importantes leis física de conservação de massa, momento e energia [28]. A seguir postulamos tais equações, cuja obtenção é mostrada de forma clara e detalhada nas Refs. [31,39].

Inicialmente consideramos as equações que descrevem conservação de massa, momento e energia, respectivamente,

$$
\begin{gathered}
\frac{d_{\alpha} n_{\alpha}}{d t}+n_{\alpha} \boldsymbol{\nabla} \cdot \mathbf{v}_{\alpha}=0 \\
m_{\alpha} n_{\alpha} \frac{d_{\alpha} \mathbf{v}_{\alpha}}{d t}+\nabla p_{\alpha}+\nabla \cdot \boldsymbol{\pi}_{\alpha}-e_{\alpha} n_{\alpha}\left(\boldsymbol{E}+\mathbf{v}_{\alpha} \times \mathbf{B}\right)=\mathbf{R}_{\alpha} \\
\frac{d_{\alpha} p_{\alpha}}{d t}+\gamma p_{\alpha} \boldsymbol{\nabla} \cdot \mathbf{v}_{\alpha}+(\gamma-1)\left(\boldsymbol{\pi}_{\alpha}: \nabla \mathbf{v}_{\alpha}+\nabla \cdot \mathbf{q}_{\alpha}\right)=(\gamma-1) Q_{\alpha}
\end{gathered}
$$

onde $d_{\alpha} / d t=\partial / \partial t+\mathbf{v}_{\alpha} \cdot \nabla$ é a derivada convectiva ou derivada material e $\gamma$ é o coeficiente de Poisson ou coeficiente adiabático.

O sistema composto pelas equações (2.66)-(2.68) é incompleto, pois para resolvê-lo são 
necessárias informações sobre o operador de colisões, responsáveis pelos termos $\mathbf{R}_{\alpha}$ e $Q_{\alpha}$, além das equações de evolução temporal para $\boldsymbol{\pi}$ e q. Apesar de que em muitos modelos seja possível desconsiderar tais grandezas, como no modelo da magnetohidrodinâmica ideal, há casos em que é necessário o cálculo dos próximos momento da equação de Boltzmann para obter tais grandezas. No capítulo 3 consideramos o efeito da anisotropia de pressão, descrito pelo tensor de viscosidade paralela $\left(\boldsymbol{\pi}_{\|}\right)$. Este tensor é calculado através da equação de evolução de $\boldsymbol{\pi}$,

$$
\begin{aligned}
& \frac{d \boldsymbol{\pi}_{\alpha}}{d t}+\left(\boldsymbol{\nabla} \cdot \mathbf{v}_{\alpha}\right) \boldsymbol{\pi}_{\alpha}+\left[\boldsymbol{\pi}_{\alpha} \cdot \boldsymbol{\nabla} \mathbf{v}_{\alpha}+\left(\boldsymbol{\pi}_{\alpha} \cdot \boldsymbol{\nabla} \mathbf{v}_{\alpha}\right)^{T}-(\gamma-1)\left(\boldsymbol{\pi}_{\alpha}: \nabla \mathbf{v}_{\alpha}\right) \mathbf{I}\right]- \\
& \omega_{c_{\alpha}} \hat{\mathbf{K}}\left(\boldsymbol{\pi}_{\alpha}\right)+p\left[\boldsymbol{\nabla} \mathbf{v}_{\alpha}+\left(\boldsymbol{\nabla} \mathbf{v}_{\alpha}\right)^{T}-(\gamma-1)\left(\boldsymbol{\nabla} \cdot \mathbf{v}_{\alpha}\right) \mathbf{I}\right]+ \\
& (1-1 / \gamma)\left[\boldsymbol{\nabla} \mathbf{q}_{\alpha}+\left(\boldsymbol{\nabla} \mathbf{q}_{\alpha}\right)^{T}-(\gamma-1)\left(\boldsymbol{\nabla} \cdot \mathbf{q}_{\alpha}\right) \mathbf{I}\right]+\boldsymbol{\nabla} \cdot \boldsymbol{\tau}=\mathbf{C}_{\pi_{\alpha}},
\end{aligned}
$$

que foi obtida primeiramente em $[70,71]$ no contexto de gases neutros e, posteriormente, adaptada para aplicações em física de plasmas $[37,72,73]$. Nesta equação, o índice $T$ em sobrescrito representa a transposta da matriz que se obtém na representação do termo a que este índice se refere na forma matricial, $\boldsymbol{\tau}$ e $\mathbf{C}_{\pi_{\alpha}}$ são tensores provenientes de momentos de mais alta ordem da função distribuição e do operador de colisões, os quais não são considerados nesta tese e $\hat{\mathbf{K}}$ é um operador que, de acordo com a definição em [74,75], satisfaz as seguintes propriedades:

$$
\begin{aligned}
& \hat{\mathbf{K}}(\boldsymbol{A})=\boldsymbol{A} \times \mathbf{b}-\mathbf{b} \times \boldsymbol{A} \text { e } \\
& \hat{\mathbf{K}}^{-1}(\boldsymbol{A})=\frac{1}{4}\left\{[\mathbf{b} \times \boldsymbol{A} \cdot(\mathbf{I}+3 \mathbf{b b})]+[\mathbf{b} \times \boldsymbol{A} \cdot(\mathbf{I}+3 \mathbf{b b})]^{T}\right\},
\end{aligned}
$$

para qualquer tensor simétrico $\boldsymbol{A}$. O tensor de viscosidade paralela de íons, o qual consideramos no capítulo 3, é calculado pela seguinte expressão:

$$
\boldsymbol{\pi}_{\| i}=\frac{\pi_{\| i}}{\gamma_{i}-1}\left(\mathbf{b b}-\frac{1}{3} \mathbf{I}\right)
$$




\subsubsection{Teoria da magneto-hidrodinâmica (MHD) ideal}

Para justificar o uso das equações da MHD ideal, é necessário considerar alguns comprimentos e tempos característicos importantes, conforme mostrados a seguir:

$$
L_{\mathrm{MHD}} \sim a, \quad \tau_{\mathrm{MHD}}=\frac{a}{v_{T_{i}}}, \quad \omega_{\mathrm{MHD}} \sim \frac{1}{\tau_{\mathrm{MHD}}}, \quad \lambda_{\alpha}=v_{T_{\alpha}} \tau_{\alpha \alpha}
$$

Em (2.72), $L_{\mathrm{MHD}}$ é o comprimento característico relativo a gradientes de quantidades macroscópicas, $\omega_{\text {MHD }}$ é a frequência associada a modos MHD e $\lambda_{\alpha}$ é o livre caminho médio, o qual depende da velocidade térmica e do tempo de colisões de partículas de mesmo tipo.

Apesar de que a teoria da MHD, do ponto de vista teórico, se aplique somente nas seguintes circunstâncias:

$$
\begin{aligned}
& \left(\frac{m_{i}}{m_{e}}\right)^{1 / 2} \frac{\omega_{\mathrm{MHD}}}{\nu_{\mathrm{ii}}} \ll 1 \quad \text { (plasma altamente colisional), } \\
& \frac{\rho_{i}}{a} \ll 1 \quad \text { (raio de Larmor muito pequeno) }, \\
& \left(\frac{\rho_{i}}{a}\right)^{2}\left(\frac{m_{i}}{m_{e}}\right)^{1 / 2} \frac{\nu_{\mathrm{ii}}}{\omega_{\mathrm{MHD}}} \ll 1 \quad \text { (plasma de baixa resistividade) }
\end{aligned}
$$

que raramente pertencem à realidade de plasmas de tokamak, o seu uso em inúmeros modelos que violam tais circunstâncias reproduz resultados condizentes com experimentos [46] e, portanto, embora a teoria da MHD ideal seja uma teoria relativamente simples, ela possue inúmeras aplicações importantes $[39,46]$.

Normalmente na teoria da MHD considera-se que o plasma é um fluido de densidade de massa $\rho$, densidade de carga $\rho_{c}$ (que no caso de caso de tokamaks é practicamente nula), pressão $p$, temperatura $T$ e corrente $\mathbf{j}$. Estas grandezas que caracterizam esse fluido, respeitam determinadas relações com as grandezas pertencentes aos fluidos de íons e de elétrons, as quais são mostradas a seguir:

$$
\rho=\sum_{\alpha} m_{\alpha} n_{\alpha} \approx m_{i} n_{i}, \quad n_{i}=n \approx n_{e}, \quad m_{e} \ll m_{i}
$$




$$
\begin{gathered}
\rho_{c}=\sum_{\alpha} e_{\alpha} n_{\alpha}=e\left(n_{i}-n_{e}\right) \approx 0, \\
\mathbf{v}=\frac{\sum_{\alpha} m_{\alpha} n_{\alpha} \mathbf{v}_{\alpha}}{\rho} \approx \mathbf{v}_{i}, \\
\mathbf{J}=\sum_{\alpha} e_{\alpha} n_{\alpha} \mathbf{v}_{\alpha} \approx n e\left(\mathbf{v}_{i}-\mathbf{v}_{e}\right), \\
p=\sum_{\alpha} p_{\alpha}=n_{i} T_{i}\left(1+\tau_{e}\right), \quad \tau_{e}=\frac{T_{e}}{T_{i}} .
\end{gathered}
$$

Note que a correspondência inversa, referente às velocidades de íons e de elétrons, pode ser escrita na forma:

$$
\mathbf{v}_{i} \approx \mathbf{v} \quad \text { e } \quad \mathbf{v}_{e} \approx \mathbf{v}-\frac{\mathbf{J}}{e n}
$$

A resistividade do plasma, que é um importante parâmetro na teoria da MHD, depende principamente da frequência de colisões elétron-íon e, normalmente, é definida como [39]:

$$
\eta=\frac{m_{e} \nu_{\mathrm{ei}}}{e^{2} n}
$$

Combinações lineares das equações (2.66)-(2.68) ponderadas por grandezas características de íons ou elétrons, cujos detalhes algébricos podem ser encontrados em [31,39,46], permitem obter o conjunto de equações da MHD, que são apresentadas e discutidas separadamente nos parágrafos que se seguem.

Primeiramente, consideramos a equação referente a conservação de massa,

$$
\frac{d \rho}{d t}+\rho \boldsymbol{\nabla} \cdot \mathbf{v}=0
$$


cuja equação análoga, mas para conservação de carga elétrica é

$$
\frac{\partial \rho_{c}}{\partial t}+\nabla \cdot \mathbf{J}=0
$$

Na maior parte dos processos em MHD a condição de quasi-neutralidade se aplica, de forma que $\rho_{c} \approx 0$, e consequentemente

$$
\nabla \cdot \mathbf{J}=0 .
$$

A equação de conservação de momento pode ser escrita da seguinte forma:

$$
\rho \frac{d \mathbf{v}}{d t}+\nabla p-\mathbf{J} \times \mathbf{B}+\boldsymbol{\nabla} \cdot\left(\boldsymbol{\pi}_{i}+\boldsymbol{\pi}_{e}\right)-\rho_{c} \boldsymbol{E}+\frac{m_{e}}{e^{2} n}[\boldsymbol{\nabla} \cdot(\mathbf{J J})-\mathbf{J J} \cdot \boldsymbol{\nabla} \ln n]=0,
$$

na qual alguns comentários são pertinentes para justificar simplificações que permitem aplicá-la efetivamente em modelos MHD. Primeiramente, mesmo sem considerar a razoável aproximação $\left|\mathbf{v}_{i}-\mathbf{v}_{e}\right|=\mid \mathbf{J} /$ en $|\ll| \mathbf{v} \mid$, assumindo que tais termos são de mesma ordem, observa-se que em (2.84) os dois últimos termos são desprezíveis com relação ao primeiro por um fator $m_{e} / m_{i}$, visto que $|\rho d \mathbf{v} / d t| \sim \rho v^{2} / a$ e $\left|m_{e} \boldsymbol{\nabla} \cdot(\mathbf{J J}) / e^{2} n\right| \sim\left(m_{e} / m_{i}\right) \rho v^{2} / a$, a menos que ocorra um forte gradiente de densidade, $|\nabla \ln n| \sim\left(m_{i} / m_{e}\right) / a$, o que geralmente não ocorre em experimentos. Com relação ao tensor de viscosidade, cujo termo dominante é a viscosidade paralela (pois $\omega_{c_{\alpha}} \tau_{\text {ei }} \gg 1$ para plasmas magnetizados) de íons, visto que, $\boldsymbol{\pi}_{e \|} \sim\left(m_{e} / m_{i}\right)^{1 / 2} \boldsymbol{\pi}_{i \|}$, a comparação $\left|\nabla \cdot \boldsymbol{\pi}_{i}\right| /|\nabla p| \sim \omega_{\mathrm{MHD}} / \nu_{i i}$, em que $\left|\boldsymbol{\pi}_{i \|}\right| \sim p_{i} \omega_{\mathrm{MHD}} / \nu_{\mathrm{ii}}$, justifica desprezar os efeitos de viscosidade em plasmas altamente colisionais. Por fim, em se tratando de modos de baixas frequências, a condição de quasi-neutralidade pode ser considerada. Com base nestes argumentos a eq. (2.84) pode ser aproximada para a seguinte forma:

$$
\rho \frac{d \mathbf{v}}{d t}+\nabla p-\mathbf{J} \times \mathbf{B}=0 .
$$


Analogamente, a partir de (2.68) obtém-se a lei de Ohm generalizada,

$$
\begin{aligned}
& \boldsymbol{E}+\mathbf{v} \times \mathbf{B}=\frac{1}{e n}\left(\mathbf{J} \times \mathbf{B}-\boldsymbol{\nabla} p_{e}-\boldsymbol{\nabla} \cdot \boldsymbol{\pi}_{e}+\mathbf{R}_{e}\right)+ \\
& \frac{m_{e}}{e^{2} n}\left[\frac{\partial \mathbf{J}}{\partial t}+\boldsymbol{\nabla} \cdot(\mathbf{J} \mathbf{v}+\mathbf{v} \mathbf{J}-\mathbf{J J} / e n)\right] .
\end{aligned}
$$

Nesta equação, primeiramente, podemos comparar o termo de Hall, $\mathbf{J} \times \mathbf{B} /$ en com os termos entre colchetes, dos quais os três primeiros são de mesma ordem e o quarto é muito menor do que estes, pois $\mathbf{J} / e n \ll \mathbf{v}$, uma vez que $\beta^{-1} \rho_{e} / L_{\mathrm{MHD}} \ll 1$ mesmo em sistemas de baixa pressão $\left(\beta \sim \varepsilon^{2}\right)$. Desta forma, $\left|m_{e}(\partial \mathbf{J} / \partial t) / e^{2} n\right| /|\mathbf{J} \times \mathbf{B} / e n| \sim \omega_{\mathrm{MHD}} / \omega_{c_{e}}=\rho_{e} / a \ll 1$, o que mostra que os termos entre colchetes podem ser desprezados. Portanto a análise agora se restringe ao termo de Hall $(\mathbf{J} \times \mathbf{B} /$ en $)$, ao termo diamagético $\left(\nabla p_{e} /\right.$ en $)$ e ao termo de fricção $\left(\mathbf{R}_{e} /\right.$ en $)$. Os dois primeiros são de mesma ordem, pois $\nabla p_{e} \sim \mathbf{J} \times \mathbf{B}$ no equilíbrio, e podem ser desprezados na ordem MHD $\left(v_{E} \sim v_{T_{i}}\right)$, pois $\left|\nabla p_{e} / e n\right| /|\mathbf{v} \times \mathbf{B}| \sim \rho_{i} / a \ll 1$. Mesmo na ordem de deriva $\left(v_{E} \sim \delta_{\rho} v_{T_{i}}\right)$, o termo $\mathbf{J} \times \mathbf{B}-\nabla p_{e}$ pode ser muito pequeno, de forma que desprezar os termos de Hall e diamagnético simultaneamente pode ser justificável. Como $T_{i} \sim T_{e}$, o termo proveniente da viscosidade de elétrons $\left(\boldsymbol{\nabla} \cdot \boldsymbol{\pi}_{e}\right)$ também pode ser desconsiderado. Resta, finalmente, em (2.86) o termo de fricção a ser analisado, o qual pode ser expresso como:

$$
\frac{\mathbf{R}_{e}}{e n}=\eta\left(0,51 \mathbf{j}_{\|}+\mathbf{j}_{\perp}\right)-\frac{1}{2} \frac{T_{e}}{T_{i}} v_{T_{i}} B \rho_{i}\left(\mathbf{b} \nabla_{\|} \ln T_{e}+\frac{3}{2} \frac{\mathbf{b} \times \nabla \ln T_{e}}{\omega_{c_{e}} \tau_{\mathrm{ei}}}\right) .
$$

Em (2.87), os termos em parênteses podem ser desprezados quando comparados com $\mathbf{v} \times \mathbf{B}$, pois eles são de ordem $\rho_{i} / \lambda_{e} \sim \nu_{\mathrm{ei}} / \omega_{c_{i}} \ll 1$ e $\left(\rho_{i} / a\right) / \omega_{c_{e}} \tau_{\mathrm{ei}} \ll 1$, respectivamente.

A lei de Ohm com resistividade pode, então ser aproximada para

$$
\boldsymbol{E}+\mathbf{v} \times \mathbf{B}=\eta\left(0,51 \mathbf{j}_{\|}+\mathbf{j}_{\perp}\right),
$$

porém, para fenômenos que envolvam tempos característicos inferiores ao tempo de difusão do campo magnético $\left(\tau_{\mathrm{B}}=\mu_{0} a^{2} / \eta\right)$ o efeito da resistividade pode ser desprezado. Esta condição é satisfeita em muitos caso, pois $|\eta \mathbf{J}| /|\mathbf{v} \times \mathbf{B}| \sim\left(\nu_{\mathrm{ei}} / \omega_{\mathrm{MHD}}\right)\left(\rho_{e}^{2} / a^{2}\right)$, ou seja, ela só é violada em fenômenos de frequências muito baixas em plasmas altamente colisionais. Caso isto não ocorra, 
a lei $\mathbf{d e} \mathbf{O h m}$ pode ser aproximada por

$$
\boldsymbol{E}+\mathbf{v} \times \mathbf{B}=0 .
$$

Finalmente, resta analisar a última equação,

$$
\begin{aligned}
& \frac{d p}{d t}+\gamma p \boldsymbol{\nabla} \cdot \mathbf{v}-\frac{\mathbf{J} \cdot\left(\boldsymbol{\nabla} p_{e}-\gamma p_{e} \boldsymbol{\nabla} \ln \rho\right)}{e n}+ \\
& (\gamma-1)\left[\left(\boldsymbol{\pi}_{i}+\boldsymbol{\pi}_{e}\right): \boldsymbol{\nabla} \mathbf{v}+\boldsymbol{\nabla} \cdot\left(\mathbf{q}_{i}+\mathbf{q}_{e}\right)-\boldsymbol{\pi}_{e}: \boldsymbol{\nabla}(\mathbf{J} / e n)-\frac{\mathbf{J} \cdot \mathbf{R}_{e}}{e n}\right]=0
\end{aligned}
$$

que corresponde à lei de conservação de energia. Em condições normais, $\gamma p_{e} \boldsymbol{\nabla} \ln \rho \sim \nabla p_{e}$, para tokamaks e, como $\left|\mathbf{J} \cdot \nabla p_{e} / e n\right| /|d p / d t| \sim \rho_{i} / a \ll 1$, justifica-se desprezar o termo que engloba os parênteses na primeira linha de (2.90). Ao considerarmos que $\boldsymbol{\pi}_{e} \ll \boldsymbol{\pi}_{i}$ e $\mathbf{J} /$ en $\ll \mathbf{v}$, analogamente à análise de (2.84), a comparação $\left(\left|\boldsymbol{\pi}_{i}: \nabla \mathbf{v}\right| /|d p / d t| \sim \omega_{\mathrm{MHD}} / \nu_{\text {ii }}\right.$ nos leva a concluir que o termo de viscosidade pode ser desprezado em plasmas altamente colisionais. Com relação à análise da componente paralela do fluxo de calor, $q_{\| \alpha} \approx-\kappa_{\| \alpha} \nabla_{\|} T_{\alpha}$, como $\kappa_{\| i} / \kappa_{\| e} \sim\left(m_{e} / m_{i}\right)^{1 / 2} \ll 1$ e $\left|\boldsymbol{\nabla} \cdot \mathbf{q}_{e}\right| /|d p / d t| \sim \nu_{\mathrm{ei}} / \omega_{\mathrm{MHD}}$ podemos desprezar tal componente em fenômenos de considerável frequência em regimes não-colisionais. Neste trabalho, consideramos apenas plasmas sem resistividade de forma que a força de friç̧ão pode ser desprezada em (2.90) e, com relação ao fluxo de calor, apenas a componente ${ }^{12}$

$$
\mathbf{q}_{\times}=\frac{p}{e B^{2}} \mathbf{B} \times \nabla T
$$

que é sensível ao gradiente de temperatura é utilizada na equação da energia, a qual se reduz a

$$
\frac{d p}{d t}+\gamma p \boldsymbol{\nabla} \cdot \mathbf{v}+(\gamma-1) \boldsymbol{\nabla} \cdot \mathbf{q}_{\times}=0
$$

As eqs. (2.81), (2.83), (2.85), (2.89), (2.92) constituem um sistema de 9 equações escalares com 16 variáveis representadas pelo conjunto de grandezas escalares $(\rho, p, T)$ e vetoriais $(\mathbf{v}, \mathbf{J}$, B e $\boldsymbol{E})$. Desta forma, a determinação deste sistema requer mais 7 equações independentes. Seis

\footnotetext{
${ }^{12}$ Em inglês, esta componente é conhecida como "Cross heat flux"
} 
destas equações são as componentes vetoriais das seguintes equações de Maxwell:

$$
\begin{gathered}
\nabla \times \boldsymbol{E}=-\frac{\partial \mathbf{B}}{\partial t}, \\
\nabla \times \mathbf{B}=\mu_{0} \mathbf{J} .
\end{gathered}
$$

Note que a equação de Maxwell que expressa a ausência de monopolo magnético,

$$
\nabla \cdot \mathbf{B}=0
$$

não pode ser considerada uma equação independente, assim como $\boldsymbol{\nabla} \cdot \boldsymbol{E}=0$, pois (2.95) pode ser obtida pelo cálculo do divergente dos dois lados de (2.93) e pelo uso da identidade (B.4). No entanto, a equação (2.95) possui importância fundamental em física de plasmas para a determinação do campo magnético de equilíbrio, pois estabelece condições algébricas e vetoriais para o cálculo de $\mathbf{B}$ [46], conforme discutido em 2.2.

A última equação necessária para completar o sistema descrito acima é a relação entre densidade, pressão e temperatura que, conforme as definições anteriores das quantidades macroscópicas para um fluido, pode ser expressa como

$$
p \approx \frac{\rho}{m_{i}} T .
$$

Em muitos casos, assim como no presente trabalho desta tese, é conveniente expressar $\boldsymbol{E}$ e B em termos de potenciais, ou seja,

$$
\boldsymbol{E}=-\nabla \Phi-\frac{\partial \boldsymbol{A}}{\partial t}, \quad \mathbf{B}=\boldsymbol{\nabla} \times \boldsymbol{A}
$$




\section{Capítulo 3}

\section{Modelo de fluido para fluxos zonais e}

\section{modos acústicos geodésicos}

Neste capítulo utilizamos a teoria da magneto-hidrodinâmica (MHD) ideal e um modelo de dois fluidos que inclui viscosidade paralela de íons para obter a frequência de modos geodésicos de baixas frequências. A dinâmica destes modos, pioneiramente descobertos por N. Winsor et. al [26] é descrita na seção 3.4. Ao investigar o equilíbrio com rotação poloidal e toroidal, tendo como base o trabalho desenvolvido por V. N. Ilgisonis [50], em 2011, obtivemos relações entre o gradiente de temperatura e a rotação poloidal. Considerando a contribuição do fluxo de calor proveniente do gradiente radial de temperatura obtivemos, no regime isotérmico, além das duas soluções correspondentes a modos acústicos geodésicos (GAM) e o modo acústico de íon (SW), a correção para a frequência dos fluxos zonais $(\mathrm{ZF})$, a qual é sensível à rotação poloidal, mas não à rotação toroidal. Tal resultado foi publicado recentemente [76]. Com relação ao modelo de dois fluidos, primeiramente estudamos o efeito de anisotropia de pressão de íons através da equação de evolução temporal da viscosidade paralela. Este efeito, quando considerado na dinâmica dos GAM, produz uma sensível diferença no valor para a frequência destes [29,36]. Posteriormente incluímos neste modelo efeitos diamagnéticos, os quais são provenientes de gradientes de temperatura de íons e de densidade. As condições para instabilidade dos GAM, devido a estes gradientes, as quais foram publicadas recentemente em 2013 [77], são descritas na seção 
3.7. Apresentamos, no final, como proposta para trabalhos futuros, uma breve discussão sobre efeitos eletromagnéticos nos GAM. Esta discussão é feita dentro do contexto da teoria de dois fluidos.

\subsection{Modelo da magnetohidrodinâmica (MHD) ideal}

Como ponto de partida para este capítulo, utilizamos a teoria da MHD ideal considerando o plasma como sendo composto por um único fluido, que, por sua vez, tem sua dinâmica governada pelas equações (2.81), (2.83), (2.85), (2.89) e (2.92) apresentadas anteriormente na seção 2.6.2. Abaixo, para facilitar a leitura, repetimos tais equações, porém, acrescentando o índice " $\Sigma$ ", que indica a soma das partes de equilíbrio (estacionária) e perturbada (dependente do tempo) das grandezas macroscópicas do plasma:

$$
\begin{gathered}
\boldsymbol{E}_{\Sigma}+\mathbf{v}_{\Sigma} \times \mathbf{B}_{\Sigma}=0 \\
\rho_{\Sigma} \frac{d \mathbf{v}_{\Sigma}}{d t}+\nabla p_{\Sigma}-\mathbf{J}_{\Sigma} \times \mathbf{B}_{\Sigma}=0 \\
\frac{d p_{\Sigma}}{d t}+\gamma p_{\Sigma} \boldsymbol{\nabla} \cdot \mathbf{v}_{\Sigma}+(\gamma-1) \nabla \cdot \mathbf{q}_{\Sigma}=0 \\
\frac{d \rho_{\Sigma}}{d t}+\rho \boldsymbol{\nabla} \cdot \mathbf{v}_{\Sigma}=0 \\
\nabla \cdot \mathbf{J}_{\Sigma}=0 .
\end{gathered}
$$

O índice $\Sigma$ é utilizado para simplificar a notação do conteúdo que se segue após a linearização das equações (3.1)-(3.5) por meio da teoria de perturbações. Nesta teoria, as grandezas macroscópicas do plasma, $p_{\Sigma}, \rho_{\Sigma}$ e as componentes vetoriais de $\boldsymbol{E}_{\Sigma}, \mathbf{B}_{\Sigma}, \mathbf{J}_{\Sigma}$, $\mathbf{v}_{\Sigma}$ e $\mathbf{q}_{\Sigma}$ são consideradas como sendo compostas por uma parte estacionária e por uma pequena perturbação, em módulo, 
dependente do tempo, de forma que,

$$
X_{\Sigma}=X_{\Sigma}(\mathbf{r}, t)=X(\mathbf{r})+\tilde{X}(\mathbf{r}) \mathrm{e}^{-i \omega t}, \quad \frac{|\tilde{X}|}{|X|} \ll 1,
$$

onde $X_{\Sigma}$ representa qualquer grandeza macroscópica (ou uma de suas componentes vetoriais) do plasma. Adotamos também o símbolo " " para indicar as quantidades perturbardas.

Restringimos o estudo desta seção ao caso de plasmas com $\beta=\mathcal{O}\left(\varepsilon^{2}\right)$ e com velocidade de

equilíbrio subsônica, $|\mathbf{V}|^{2} \ll c_{s}^{2}$, de forma que perturbações do campo magnético, $\tilde{\mathbf{B}}=\mathcal{O}(\beta \mathbf{B})$, podem ser desprezadas na análise de apenas primeiros harmônicos, $m= \pm 1$. Sendo assim, apenas o potencial eletrostático é considerado em nossa análise de modos de baixas frequências, ou seja, $\boldsymbol{E}_{\Sigma}=-\nabla \Phi-\nabla \tilde{\Phi}$.

\subsection{Equilíbrio com rotação}

Considerando a ordem MHD $\left(v_{E} \sim v_{T_{i}}\right)$, de forma que o efeito Hall e a deriva diamagnética podem ser desprezados na lei de Ohm, o equilíbrio é descrito pelas equações:

$$
\begin{gathered}
\mathbf{V} \times \mathbf{B}=-\nabla \Phi, \\
\mathbf{V} \cdot \boldsymbol{\nabla} \rho+\rho \boldsymbol{\nabla} \cdot \mathbf{V}=0, \\
\mathbf{V} \cdot \boldsymbol{\nabla} p+\gamma p \boldsymbol{\nabla} \cdot \mathbf{V}+(\gamma-1) \boldsymbol{\nabla} \cdot \mathbf{q}=0, \\
\rho \mathbf{V} \cdot \boldsymbol{\nabla} \mathbf{V}+\nabla p-\mathbf{J} \times \mathbf{B}=0,
\end{gathered}
$$

onde

$$
\mathbf{q}=\frac{\gamma}{\gamma-1} \frac{p \mathbf{B} \times \nabla T}{e B^{2}}
$$


é a parte dominante do fluxo de calor no caso não colisional, a qual deve ser considerada no estudo de ZF e na investigação de efeitos causado pelo gradiente de temperatura.

Assumimos que o campo magnético é simétrico em relação ao ângulo toroidal $(\phi)$, de forma que

$$
\begin{gathered}
\mathbf{B}=F \nabla \phi+\nabla \phi \times \nabla \Psi, \quad \nabla \Psi \cdot \nabla \phi=0, \\
\mathbf{J}=\frac{\boldsymbol{\nabla} \times \mathbf{B}}{\mu_{0}}=\frac{\left(R^{2} \Delta^{*} \Psi \nabla \phi-\nabla \phi \times \nabla F\right)}{\mu_{0}}, \quad \Delta^{*} \Psi=\nabla \cdot\left(\nabla \Psi / R^{2}\right),
\end{gathered}
$$

conforme mostrado em C.1.

Das eqs. (3.7) e (3.8) segue que

$$
\mathbf{V}=\frac{\kappa(\Psi)}{\rho} \mathbf{B}-\Omega(\Psi) R^{2} \nabla \phi, \quad \Omega=\frac{d \Phi}{d \Psi}
$$

onde $\kappa$ é uma função de fluxo desconhecida, porém que está diretamente relacionada à rotação poloidal de equilíbrio. Com a substituição de $\mathbf{V}$ em (3.9), segue que

$$
\frac{\kappa}{\rho} \mathbf{B} \cdot \nabla p+\gamma p \mathbf{B} \cdot \nabla\left(\frac{\kappa}{\rho}\right)+(\gamma-1) \boldsymbol{\nabla} \cdot \mathbf{q}=0
$$

e, portanto, observa-se que na ausência de rotação poloidal $(\kappa=0)$, o fluxo de calor tem divergência nula, ou seja, em média não há troca de calor entre as superfícies magnétcias.

A relação entre pressão, densidade e temperatura, $p=\rho T / m_{i}$, pode ser convenientemente expressa, para uso futuro, como:

$$
\frac{\mathbf{B} \cdot \boldsymbol{\nabla} \rho}{\rho}-\frac{\mathbf{B} \cdot \boldsymbol{\nabla} p}{p}+\frac{\mathbf{B} \cdot \boldsymbol{\nabla} T}{T}=0
$$

O método algébrico pelo qual os resultados anteriores e os próximos foram obtidos é apresentado no apêndice C, cujo principal objetivo é elucidar a obtenção das expressões algébricas para as componentes $\boldsymbol{\nabla} \phi, \mathbf{B}$ e $\boldsymbol{\nabla} \Psi$ da equação de momento. Tais componentes, identicamente nulas, são obtidas pelo cálculo do produto escalar de $\nabla \phi, \mathbf{B}$ e $\nabla \Psi$ com a eq.(3.10), e podem 
ser expressas como:

$$
\begin{gathered}
\mathbf{B} \cdot \boldsymbol{\nabla}\left[F\left(1-\frac{\mu_{0} \kappa^{2}}{\rho}\right)+\mu_{0} \kappa \Omega R^{2}\right]=0 \\
\mathbf{B} \cdot \boldsymbol{\nabla}\left(\frac{\kappa^{2} B^{2}}{2 \rho^{2}}-\frac{\Omega^{2} R^{2}}{2}\right)+\frac{\mathbf{B} \cdot \boldsymbol{\nabla} p}{\rho}=0 \\
\left(1-\frac{\mu_{0} \kappa^{2}}{\rho}\right) \Delta^{*} \Psi+\frac{1}{2} \frac{\nabla \Psi \cdot \nabla F^{2}}{|\nabla \Psi|^{2}}+\frac{\mu_{0} R^{2}}{|\nabla \Psi|^{2}} \nabla \Psi \cdot \nabla p+\frac{\mu_{0} \rho R^{2}}{2} \times \\
{\left[\frac{\nabla \Psi}{|\nabla \Psi|^{2}} \cdot \nabla\left(\frac{\kappa^{2}}{\rho^{2}} \frac{|\nabla \Psi|^{2}}{R^{2}}\right)-\frac{\nabla \Psi}{R^{2}} \cdot \nabla\left(\frac{\kappa^{2}}{\rho^{2}}\right)-\left(\Omega-\frac{\kappa F}{\rho R^{2}}\right)^{2} \frac{\nabla \Psi \cdot \nabla R^{2}}{|\nabla \Psi|^{2}}\right]=0,}
\end{gathered}
$$

onde $\Delta^{*} \Psi=R^{2} \nabla \cdot\left(\nabla \Psi / R^{2}\right)$ é o operador de Shafranov.

Observando que se $\mathbf{B} \cdot \boldsymbol{\nabla} f=0$, para qualquer função escalar $f$ independente de $\phi$, implica em $f=f(\Psi)$, conclui-se que somente na ausência de rotação poloidal $(\kappa=0)$, de acordo com (3.17), então $F=F(\Psi)$. Ainda, neste mesmo contexto, se considerarmos o caso de rotação exclusivamente toroidal, de acordo com (3.18), $\mathbf{B} \cdot \boldsymbol{\nabla} p=\rho \Omega \mathbf{B} \cdot \boldsymbol{\nabla} R^{2} / 2$. Entretanto, como $\mathbf{B} \cdot \boldsymbol{\nabla} R^{2} \neq 0$, conclui-se que $p$ não pode ser uma função de fluxo devido a correção da força centrífuga devido à rotação, ao contrário do que ocorre em plasmas sem rotação, onde $p=p(\Psi)$.

O próximo passo é a utilização de teoria de perturbação para resolver as eqs. (3.15)-(3.18). Nos baseamos no método apresentado na Ref. [50], na qual as grandezas de equilíbrio são decompostas na forma: $Q=Q_{0}(\Psi)+Q_{1}(\Psi, \theta)$, com $\left|Q_{1} / Q_{0}\right| \ll 1$, onde $Q$ representa $p, \rho, T$ ou F. Definimos, então, por conveniência, a grandeza:

$$
\Delta_{Q}=\frac{\left(\mathbf{B} \cdot \nabla Q_{1}\right) / Q_{0}}{\left(\mathbf{B} \cdot \nabla R^{2}\right) / R_{0}^{2}}
$$

A frequência angular de rotação poloidal e toroidal é calculada por:

$$
\Omega_{P}=\nabla \theta \cdot \mathbf{V}=\frac{\kappa F}{\rho q R^{2}}, \quad \Omega_{T}=\nabla \phi \cdot \mathbf{V}=q \Omega_{P}-\Omega,
$$


onde $q$ é o fator de segurança, que é definido por

$$
q=q(\Psi)=\frac{\boldsymbol{\nabla} \phi \cdot \mathbf{B}}{\nabla \theta \cdot \mathbf{B}}=\frac{F}{J R^{2}}, \quad J=\nabla \theta \cdot(\boldsymbol{\nabla} \phi \times \nabla \Psi) .
$$

Por conveniência, nas equações que se seguem, introduzimos as seguintes definições:

$$
\begin{gathered}
M_{P}=\frac{q \Omega_{P 0} R_{0}}{c_{s}}, \quad M_{T}=\frac{\Omega_{T 0} R_{0}}{c_{s}}, \quad M_{\mathrm{th}}=\frac{R_{0}}{e c_{s}} \frac{d T_{0}}{d \Psi}, \quad c_{s}^{2}=\frac{\gamma p_{0}}{\rho_{0}}, \\
\Omega_{P 0}=\frac{\kappa F_{0}}{\rho_{0} q R_{0}^{2}}, \quad \Omega_{T 0}=q \Omega_{P 0}-\Omega, \quad \mathcal{B}_{0}=\frac{\mu_{0} \rho_{0} c_{s}^{2} R_{0}^{2}}{F_{0}^{2}} \sim \beta .
\end{gathered}
$$

que são relativas aos números de Mach poloidal, toroidal e térmico e ao parâmetro $\beta$.

A partir de (3.20), (3.23) e (3.24) e do cálculo da divergência do fluxo de calor,

$$
\begin{gathered}
\boldsymbol{\nabla} \cdot \mathbf{q}=M_{\mathrm{th}}\left[\frac{1-\Delta_{F}+\Delta_{p}-\left(1+\mathcal{R}_{\rho}-\mathcal{R}_{F}+\mathcal{R}_{R^{2}}\right) \Delta_{T}}{(\gamma-1) F_{0} / R_{0}}\right] \frac{\mathbf{B} \cdot \nabla R^{2}}{R_{0}^{2}} \rho_{0} c_{s}^{3}, \\
\mathcal{R}_{F}=\frac{T_{0}}{F_{0}} \frac{d F_{0} / d \Psi}{d T_{0} / d \Psi}, \quad \mathcal{R}_{\rho}=\frac{T_{0}}{\rho_{0}} \frac{d \rho_{0} / d \Psi}{d T_{0} / d \Psi}, \quad \mathcal{R}_{R^{2}}=\frac{T_{0}}{R_{0}^{2}} \frac{\nabla \Psi \cdot \nabla R^{2}}{\nabla \Psi \cdot \nabla T_{0}},
\end{gathered}
$$

que é efetuado em C.3, podemos reescrever o sistema (3.15) - (3.18) da seguinte forma:

$$
\begin{gathered}
\Delta_{\rho}-\Delta_{p}+\Delta_{T}=0 \\
\left(1-\mathcal{B}_{0} M_{P}^{2}\right) \Delta_{F}+\mathcal{B}_{0} M_{P}^{2} \Delta_{\rho}=\mathcal{B}_{0} M_{P}\left(M_{T}-M_{P}\right), \\
M_{P}^{2} \Delta_{F}-M_{P}^{2} \Delta_{\rho}+\frac{\Delta_{p}}{\gamma}=\frac{M_{T}^{2}}{2}-M_{P} M_{T}+M_{P}^{2}, \\
M_{\mathrm{th}} \Delta_{F}+M_{P} \Delta_{\rho}-\left(M_{P} / \gamma+M_{\mathrm{th}}\right) \Delta_{p}+\left(1+\mathcal{R}_{\rho}-\mathcal{R}_{F}+\mathcal{R}_{R^{2}}\right) M_{\mathrm{th}} \Delta_{T}=M_{\mathrm{th}} .
\end{gathered}
$$


Referente a equação de Grad-Shafranov modificada, (3.19), podemos reescreve-la como:

$$
\Delta^{*} \Psi+\left[\frac{\mathcal{B}_{0} R^{2}}{\gamma R_{0}^{2}}\left(1+\mathcal{R}_{\rho}\right)+\mathcal{R}_{F}\right] \frac{F_{0}^{2}}{T_{0}} \frac{d T_{0}}{d \Psi}+\mathcal{T}(\kappa, \Omega, \Psi)
$$

onde $\mathcal{T}=\mathcal{O}\left(\mathcal{B}_{0}^{2} F_{0} / L_{T}\right)$ é o termo proveniente da rotação de equilíbrio, o qual pode ser aproximado por

$$
\begin{aligned}
& \mathcal{T} \approx-\mathcal{B}_{0} M_{P}^{2} \Delta^{*} \Psi+\left[\frac{\nabla \Psi \cdot \nabla p_{1}}{\nabla \Psi \cdot \nabla p_{0}} \frac{\mathcal{B}_{0} R^{2}}{\gamma R_{0}^{2}}\left(1+\mathcal{R}_{\rho}\right)+\right. \\
& \left.\left(\frac{\nabla \Psi \cdot \nabla F_{1}}{\nabla \Psi \cdot \nabla F_{0}}-\frac{F_{1}}{F_{0}}\right) \mathcal{R}_{F}+\frac{\mathcal{B}_{0}}{2}\left(\frac{|\nabla \Psi|^{2}}{F_{0}^{2}} M_{P}^{2} \mathcal{R}_{\Psi^{2}}-M_{T}^{2}\right)\right] \frac{F_{0}^{2}}{T_{0}} \frac{d T_{0}}{d \Psi}, \\
& \mathcal{R}_{\Psi^{2}}=\frac{T_{0}}{|\nabla \Psi|^{4}} \frac{\nabla \Psi \cdot \nabla\left(|\nabla \Psi|^{2}\right)}{d T_{0} / d \Psi} \sim \frac{T_{0}}{|\nabla \Psi|^{2}} \frac{\partial|\nabla \Psi|^{2} / \partial \Psi}{d T_{0} / d \Psi} .
\end{aligned}
$$

A menos que ocorra um forte cizalhamento radial do campo magnético poloidal, ou seja, se $\partial^{2} \Psi / \partial r^{2} \gg(\partial \Psi / \partial r)^{2}$, é condizente com a realidade de tokamaks em regimes de baixa pressão $\left(\beta \sim \varepsilon^{2}\right)$, estimar as grandezas apresentadas em (3.31) da seguinte forma:

$$
\mathcal{B}_{0} \sim \varepsilon^{2}, \quad \Delta^{*} \Psi \sim \mathcal{B}_{0} \frac{F_{0}^{2}}{T_{0}} \frac{d T_{0}}{d \Psi} \sim \frac{\sqrt{\mathcal{B}_{0}} F_{0}}{L_{T}}, \quad \frac{1}{L_{T}}=\frac{1}{T_{0}} \frac{\partial T_{0}}{\partial r}
$$

o que implica em $\mathcal{R}_{F} \sim \mathcal{B}_{0}$ e $\mathcal{R}_{\rho} \approx \eta^{-1} \sim 1$, onde $\eta=L_{\rho} / L_{T}, L_{\rho}=\rho_{0}^{-1} \partial \rho_{0} / \partial r$. Com relação ao termo $\mathcal{R}_{R^{2}}$, definido em (3.26), para a estimativa de sua ordem de grandeza, consideramos tokamaks de seção circular, como o TCABR, por exemplo, de forma que $\partial \Psi / \partial \theta \ll r \partial \Psi / \partial r$. Ainda, neste contexto, quando $L_{T} \leq r$, ou seja, quando há um considerável gradiente radial de temperatura no tokamak, o que é totalmente realístico na prática, segue que

$$
\mathcal{R}_{R^{2}}=\frac{T_{0}}{R_{0}^{2}} \frac{\partial R^{2} / \partial \Psi}{d T_{0} / d \Psi} \approx 2 \frac{L_{T}}{R_{0}} \cos \theta \sim \varepsilon \ll 1
$$

Quanto maior for o gradiente de temperatura, mais justificável se torna a aproximação (3.34), o que viabiliza e simplifica o desenvolvimento de um modelo analítico. 


\subsubsection{Rotação toroidal}

Para o caso particular de rotação puramente toroidal, $M_{P}=0$, considerando as aproximações mencionadas acima, o sistema composto pelas equações (3.27) - (3.29) apresenta a seguinte solução:

$$
\Delta_{F}=0, \quad \Delta_{p}=\frac{\gamma}{2} M_{T}^{2}, \quad \Delta_{\rho}=\Delta_{p}-\Delta_{T}
$$

Com relação à análise da eq. (3.30), é necessário ter em mente as eqs. (3.15) e (3.25), que permitem concluir que $\boldsymbol{\nabla} \cdot \mathbf{q}=0$ quando não há rotação poloidal $(\kappa=0)$. Porém, de acordo com (3.25), isto só ocorre em dois casos, $\Delta_{T}=\left(1+\Delta_{p}\right) /\left(1+\mathcal{R}_{\rho}\right)$ ou $M_{\mathrm{th}}=$ 0. O primeiro caso, implicaria que no limite sem rotação de equilíbrio $\left(M_{T} \rightarrow 0\right)$, tanto a temperatura quanto a densidade de equilíbrio dependeriam fortemente com a posição poloidal, pois $\Delta_{\rho}=-\Delta_{T}=-\left(1+\mathcal{R}_{\rho}\right)^{-1} \sim 1$, em desacordo com o equilíbrio sem rotação, no qual $\Delta_{p}=\Delta_{\rho}=\Delta_{T}=0$ [26]. O segundo caso, entretanto, implica que, pelo menos em primeira ordem, a temperatura é constante em superfícies magnéticas diferentes, de acordo com (3.23), o que também não ocorre em tokamaks (a temperatura é máxima no centro e nula na borda). Uma forma de conciliar esta inconsistência é assumir que $M_{\mathrm{th}} \propto M_{P}$, ou, de forma equivalente, que a rotação poloidal de equilíbrio é uma consequência direta da existência de gradientes radiais de temperatura. Portanto, neste modelo, concluímos que a não existência de rotação poloidal só é possível localmente e, se isso ocorrer em determinada posição radial, há uma indicação clara de que nesta posição ocorre um perfil plano no perfil da temperatura.

Os seguintes regimes de particular interesse podem ser considerados neste caso:

- Adiabático: Neste caso, a quantidade $S=p \rho^{-\gamma}$, que representa a entropia do sistema, é uma função de fluxo, de forma que a relação $\Delta_{p}-\gamma \Delta_{\rho}^{(S)}=0$ se verifica. A solução correspondente a este regime é:

$$
\Delta_{p}=\frac{\gamma}{2} M_{T}^{2}, \quad \Delta_{\rho}^{(S)}=\frac{1}{2} M_{T}^{2}, \quad \Delta_{T}^{(S)}=(\gamma-1) M_{T}^{2}
$$

- Isotérmico: Caracterizado por ser o regime mais realístico, ocorre quando $\Delta_{T}^{(T)}=0$, o 
que implica na solução

$$
\Delta_{\rho}^{(T)}=\Delta_{p}
$$

- Isométrico: Este regime, caracterizado por $\Delta_{\rho}^{(V)}=0$, embora não seja comum em experimentos, tem certa importância por ser o único regime característico com ZF instáveis, conforme elucidado mais adiante. A solução correspondente é:

$$
\Delta_{T}^{(V)}=\Delta_{p}
$$

\subsubsection{Rotação poloidal e toroidal}

Com a resolução do sistema (3.27)-(3.30), considerando $\mathcal{B}_{0} \sim \varepsilon^{2} \ll 1, \mathcal{R}_{\rho} \approx 1 / \eta$ e $M_{P, T}^{2} \ll 1$, de forma que $\Delta_{F}=\mathcal{O}\left(\mathcal{B}_{0} M_{P, T}^{2}\right)$ pode ser desprezado, obtemos a seguinte solução:

$$
\begin{gathered}
\Delta_{\rho}=\frac{N_{\Delta}}{D_{\Delta}}\left[1+\left(\frac{1}{N_{\Delta}}-\frac{\gamma}{\eta}\right) \frac{M_{\mathrm{th}}}{M_{P}}\right], \\
\Delta_{p}=\gamma \frac{N_{\Delta}}{D_{\Delta}}\left[1+\left(\frac{M_{P}^{2}}{N_{\Delta}}-\frac{\eta+1}{\eta}\right) \frac{M_{\mathrm{th}}}{M_{P}}\right], \\
\Delta_{T}=(\gamma-1) \frac{N_{\Delta}}{D_{\Delta}}\left[1-\left(\frac{1-\gamma M_{P}^{2}}{(\gamma-1) N_{\Delta}}+\frac{\gamma}{\gamma-1}\right) \frac{M_{\mathrm{th}}}{M_{P}}\right],
\end{gathered}
$$

onde

$$
N_{\Delta}=\frac{M_{T}^{2}}{2}+M_{P}\left(M_{P}-M_{T}\right), \quad D_{\Delta}=1-M_{P}^{2}-\frac{\eta+1}{\eta} \frac{M_{\mathrm{th}}}{M_{P}}+\frac{\gamma}{\eta} M_{P} M_{\mathrm{th}}
$$

Assim como no caso de rotação exclusivamente toroidal, neste caso, também é conveniente analisar os três regimes principais mencionados anteriormente: 
- Adiabático: Considera-se, neste regime, $M_{\mathrm{th}}^{(S)}=0$, o que resulta em

$$
\Delta_{p}^{(S)}=\gamma \Delta_{\rho}^{(S)}, \quad \Delta_{T}^{(S)}=(\gamma-1) \Delta_{\rho}^{(S)}, \quad \Delta_{\rho}^{(S)}=\frac{N_{\Delta}}{D_{\Delta}^{(S)}}, \quad D_{\Delta}^{(S)}=1-M_{P}^{2}
$$

- Isotérmico: As soluções são obtidas pela substituição $\Delta_{T}=0$ em (3.39), (3.40) e (3.41), de forma que, para $M_{P} \geq 0$,

$$
M_{\mathrm{th}}^{(T)}=\frac{(\gamma-1) M_{P} N_{\Delta}}{1+\gamma\left(N_{\Delta}-M_{P}^{2}\right)}>0
$$

- Isométrico: De forma análoga ao regime anterior, a partir da condição $\Delta_{\rho}=0$, para $M_{P} \geq 0$, obtém-se:

$$
M_{\mathrm{th}}^{(V)}=\frac{-M_{P} N_{\Delta}}{1-(\gamma / \eta) N_{\Delta}}<0
$$

Para o tokamak TCABR, conforme apresentado no recente relatório [78], cujo estudo de rotação residual em plasmas foi baseado no trabalho de J. H. F. Severo, et. al em 2003 [79] mostramos na figura 3.1 o perfil radial da velocidade de rotação de equilíbrio obtido experimentalmente neste tokamak. A partir deste gráfico podemos estimar os valores de $M_{P}$ e $M_{T}$ com o intuito de calcular a frequência dos GAM, SW e ZF. Observe que o valor da velocidade de rotação poloidal próximo da borda da coluna de plasma previsto pela teoria neoclássia para plasmas colisionais está em desacordo com o resultado obtido experimentalmente no TCABR.

É interessante observar o que ocorre no limite $M_{T} \rightarrow 0$, ou seja, de acordo com a figura 3.1, próximo de $r=0.7 a$. Neste limite observa-se que

$$
M_{\mathrm{th}}^{(V)}=-M_{P}^{3}, \quad M_{\mathrm{th}}^{(S)}=0, \quad M_{\mathrm{th}}^{(T)}=(\gamma-1) M_{P}^{3} .
$$

Considerando finalmente, tokamaks de seç̧ão circular de alta razão de aspecto, é possível encontrar as grandezas de equilíbrio. Para uma grandeza genérica $Q$, simétrica em relação a $\phi$, 

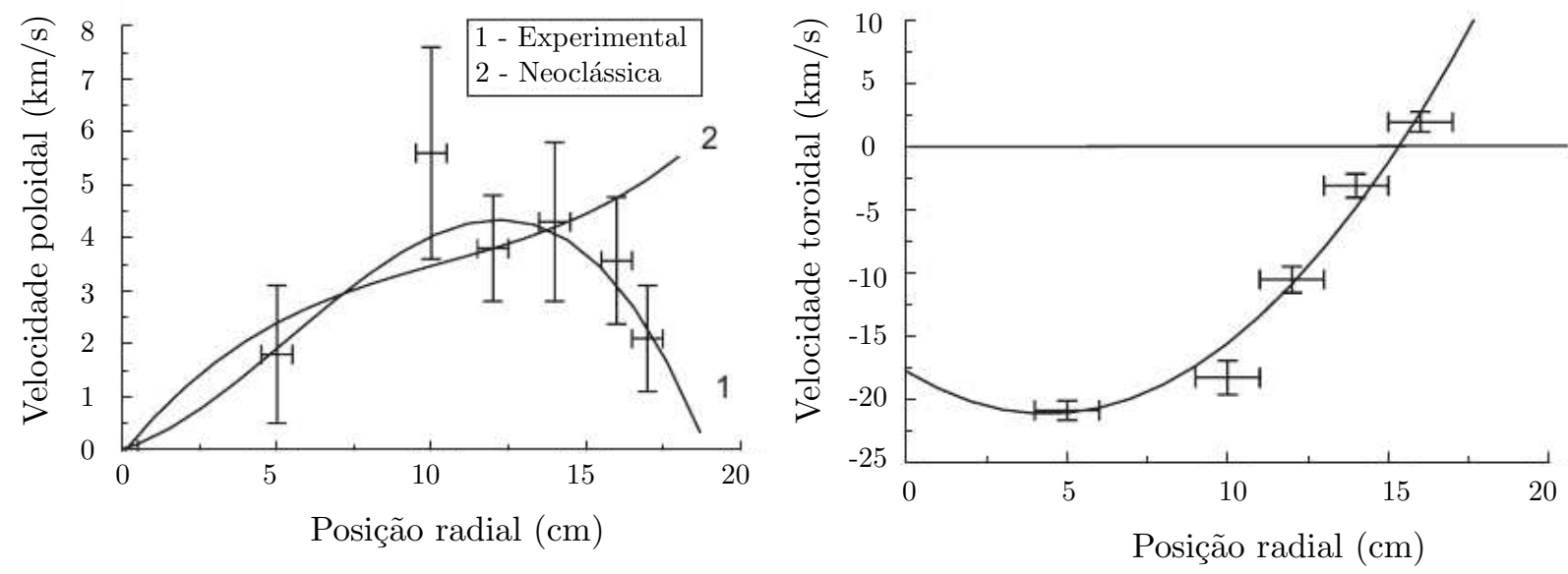

Figura 3.1: Perfil radial da velocidade de rotação de equilíbrio poloidal (esquerda) e toroidal (direita) no tokamaks TCABR. Para o caso de rotação poloidal o perfil experimental (1) foi comparado com o perfil teórico (2) obtido através da teoria neoclássica. Esta figura é uma adaptação das figuras 7 e 8 do trabalho original de J. H. F. Severo et. al [79]

segue da definição de $\Delta_{Q},(3.20)$, que

$$
\mathbf{B} \cdot \nabla Q=\Delta_{Q} Q_{0} \frac{\mathbf{B} \cdot \nabla R^{2}}{R_{0}^{2}}
$$

pode ser desenvolvido considerando $\Psi \approx \Psi(r)$, ou seja, $\mathbf{B} \approx F(r) R^{-1} \hat{\mathbf{e}}_{\phi}+(R r)^{-1}(d \Psi / d r) \hat{\mathbf{e}}_{\theta}$. Resulta então, da substituição de B em (3.47), a seguinte equação integrável,

$$
\frac{\partial Q}{\partial \theta}=-2 \varepsilon \Delta_{Q} Q_{0} \sin \theta+\mathcal{O}\left(\varepsilon^{2} Q\right)
$$


cuja solução aproximada determina $Q=Q(r, \theta)$ :

$$
Q(r, \theta)=Q_{0}(r)+2 \varepsilon \Delta_{Q}(r) Q_{0}(r) \cos \theta
$$

A partir de (3.39), (3.40), (3.41) e (3.49) a dependência poloidal das quantidades de equilíbrio podem ser determinadas, ou seja,

$$
\begin{aligned}
& \rho=\rho_{0}\left(1+2 \varepsilon \Delta_{\rho} \cos \theta\right), \quad p=p_{0}\left(1+2 \varepsilon \Delta_{p} \cos \theta\right) \\
& T=T_{0}\left(1+2 \varepsilon \Delta_{T} \cos \theta\right)=\frac{m_{i} c_{s}^{2}}{\gamma}\left[1+2 \varepsilon\left(\Delta_{p}-\Delta_{\rho}\right) \cos \theta\right] \\
& \mathbf{V}=V_{P} \hat{\mathbf{e}}_{\theta}+V_{T} \hat{\mathbf{e}}_{\phi}, \quad V_{P}=\Omega_{P} r, \quad V_{T}=\Omega_{T} R \\
& V_{P} \approx \frac{\varepsilon}{q} M_{P} c_{s}, \quad V_{T}=\left(M_{T}+\Delta_{V} \varepsilon \cos \theta\right) c_{s}, \quad \Delta_{V}=M_{T}-2 M_{P}\left(1+\Delta_{\rho}\right) .
\end{aligned}
$$

\subsection{Sistema de equações perturbadas e relação de dis-}

\section{persão}

Considerando agora perturbações temporais, os modos de oscilação de baixas frequências no plasma são obtidos a partir da resolução do seguinte sistema:

$$
\begin{gathered}
\rho_{0} \frac{\partial \tilde{v}_{\|}}{\partial t}+\nabla_{\|} \tilde{p}+\tilde{F}_{\|}=0, \\
\frac{\partial(\tilde{\rho}+\tilde{R})}{\partial t}+\rho_{0} \nabla \cdot \tilde{\mathbf{v}}=0 \\
\frac{\partial(\tilde{p}+\tilde{P})}{\partial t}+\gamma p_{0} \nabla \cdot \tilde{\mathbf{v}}=0
\end{gathered}
$$

onde

$$
\tilde{\mathbf{v}}=\tilde{\mathbf{v}}_{E}+\tilde{v}_{\|} \mathbf{b}, \quad \tilde{\mathbf{v}}_{E}=\frac{\mathbf{b} \times \boldsymbol{\nabla} \tilde{\Phi}}{B},
$$


é a velocidade perturbada proveniente da deriva $\boldsymbol{E} \times \mathbf{B}$ e da componente paralela. Os termos $\tilde{F}_{\|}, \tilde{R}$ e $\tilde{P}$ são as contribuições devidas a rotação de equilíbrio, definidos de forma conveniente por

$$
\begin{gathered}
\tilde{F}_{\|}=\rho_{0}(\mathbf{b} \tilde{\mathbf{v}}: \boldsymbol{\nabla} \mathbf{V}+\mathbf{b} \mathbf{V}: \nabla \tilde{\mathbf{v}})+\tilde{\rho} \mathbf{b} \mathbf{V}: \boldsymbol{\nabla} \mathbf{V}, \\
\frac{\partial \tilde{R}}{\partial t}=\mathbf{V} \cdot \boldsymbol{\nabla} \tilde{\rho}+\tilde{\mathbf{v}} \cdot \boldsymbol{\nabla} \rho_{0}+\tilde{\rho} \boldsymbol{\nabla} \cdot \mathbf{V}, \\
\frac{\partial \tilde{P}}{\partial t}=\mathbf{V} \cdot \boldsymbol{\nabla} \tilde{p}+\tilde{\mathbf{v}} \cdot \boldsymbol{\nabla} p_{0}+\gamma \tilde{p} \boldsymbol{\nabla} \cdot \mathbf{V}+(\gamma-1) \boldsymbol{\nabla} \cdot \tilde{\mathbf{q}},
\end{gathered}
$$

e calculadas em E.2.2, E.2.3 e E.2.5. Neste cálculo levamos em conta apenas os termos dominantes com relação ao fator $\varepsilon=r / R_{0} \ll 1$, que são devidos à contribuição dos primeiros harmônicos.

Para a obtenção da relação de dispersão é necessária a utilização da equação do momento linearizada:

$$
\rho \frac{\partial \tilde{\mathbf{v}}}{\partial t}+\nabla \tilde{p}-\tilde{\mathbf{j}} \times \mathbf{B}+\tilde{\mathbf{F}}=0, \quad \tilde{\mathbf{F}}=\rho(\mathbf{V} \cdot \nabla \tilde{\mathbf{v}}+\tilde{\mathbf{v}} \cdot \boldsymbol{\nabla} \mathbf{V})+\tilde{\rho} \mathbf{V} \cdot \nabla \mathbf{V}
$$

a qual, quando multiplicada vetorialmente por $\mathbf{B}$ resulta na expressão analítica para a densidade de corrente:

$$
\tilde{\mathbf{j}}=\frac{\tilde{j}_{\|}}{B} \mathbf{B}+\frac{\rho \mathbf{B}}{B^{2}} \times \frac{\partial \tilde{\mathbf{v}}}{\partial t}+\frac{\mathbf{B}}{B^{2}} \times \nabla \tilde{p}+\frac{\mathbf{B}}{B^{2}} \times \tilde{\mathbf{F}}
$$

A relação de dispersão é proveniente da condição de quasi-neutralidade do plasma, que pode ser expressa pela equação $\boldsymbol{\nabla} \cdot \tilde{\mathbf{j}}=0$. A metodologia analítica padrão é baseada no cálculo da média de tal equação sobre uma superfície magnética. Podemos calcular $D$ tomando a média com relação ao volume,

$$
D=\frac{\int_{V} d V \nabla \cdot \tilde{\mathbf{j}}}{\int_{V} d V}=0, \quad d V=\left(R_{0}+r \cos \theta\right) r d r d \theta d \phi
$$


e, através do teorema da divergência de Gauss, obtemos:

$$
D=\frac{\int_{S} \tilde{\mathbf{j}} \cdot d \mathbf{S}}{\int_{V} d V}=0, \quad d \mathbf{S}=\left(R_{0}+r \cos \theta\right) r d \theta d \phi \hat{\mathbf{e}}_{r}
$$

\subsection{Fluxos zonais (ZF) e modos acústicos geodésicos (GAM)}

A seguir descrevemos o modelo mais simples para explorar a dinâmica básica das oscilações eletrostáticas conhecidas como GAM. Nesta parte desconsideramos rotação de equilíbrio por motivos didáticos e com a finalidade de enfatizar o mecânismo físico de formação dos GAM. Inicialmente, utilizamos a substituição $\tilde{F}_{\|}=\tilde{P}=\tilde{R}=0$ em (3.51)-(3.53) e, como apenas os primeiros harmônicos desempenham um papel relevante na dinâmica básica dos GAM eletrostáticos [26], consideramos soluções da forma: $\tilde{X}=\tilde{X}_{s} \sin \theta+\tilde{X}_{c} \cos \theta$ para as perturbações. Ademais, em se tratando de uma análise linear, $\tilde{X} \propto \mathrm{e}^{-i \omega t}$, de forma que a substituição $\partial / \partial t \rightarrow-i \omega$ em (3.51)-(3.53) pode ser empregada.

O termo $\boldsymbol{\nabla} \cdot \tilde{\mathbf{v}}$ têm sua expressão desenvolvida no apêndice D e, de acordo com as eqs. (D.38) e (D.13), pode ser escrito na forma:

$$
\boldsymbol{\nabla} \cdot \tilde{\mathbf{v}}=-2 \tilde{\omega}_{E} \sin \theta+k_{\|} \frac{\partial \tilde{v}_{\|}}{\partial \theta}, \quad \tilde{\omega}_{E}=\frac{i \mathrm{k}_{r} \tilde{\Phi}_{0}}{B_{0} R_{0}}=\frac{i}{2} \frac{e \tilde{\Phi}_{0}}{T_{i}} \mathrm{k}_{r} \rho_{i} \omega_{i}, \quad \omega_{i}=\frac{v_{T_{i}}}{R_{0}}
$$

Tal termo é substituído em (3.53), resultando na relação entre $\tilde{p}$ e $\tilde{v}_{\|}$:

$$
\tilde{p}=i \rho_{0} c_{s}^{2}\left(-2 \frac{\tilde{\omega}_{E}}{\omega} \sin \theta-\frac{k_{\|}}{\omega} \frac{\partial \tilde{v}_{\|}}{\partial \theta}\right)
$$

que, por sua vez, é substituída em (3.51). Consequentemente, a seguinte equação diferencial para $\tilde{v}_{\|}$em $\theta$ é obtida:

$$
\left(1+\frac{k_{\|}^{2} c_{s}^{2}}{\omega^{2}} \frac{\partial^{2}}{\partial \theta^{2}}\right) \tilde{v}_{\|}=2 \frac{k_{\|} c_{s}^{2}}{\omega^{2}} \tilde{\omega}_{E} \cos \theta
$$


A solução correspondente a(3.64),

$$
\tilde{v}_{\|}=\frac{2 k_{\|} c_{s}^{2}}{\omega^{2}-k_{\|}^{2} c_{s}^{2}} \tilde{\omega}_{E} \cos \theta
$$

quando inserida em (3.62) define completamente o termo $\boldsymbol{\nabla} \cdot \tilde{\mathbf{v}}$, que, após ser substituído em (3.52) e (3.53), completa o conjunto de soluções com

$$
\begin{gathered}
\boldsymbol{\nabla} \cdot \tilde{\mathbf{v}}=-\frac{2 \omega^{2}}{\omega^{2}-k_{\|}^{2} c_{s}^{2}} \tilde{\omega}_{E} \sin \theta, \\
\tilde{\rho}=i \rho_{0}\left(\frac{2 \omega}{\omega^{2}-k_{\|}^{2} c_{s}^{2}}\right) \tilde{\omega}_{E} \sin \theta, \quad \tilde{p}=\tilde{\rho} c_{s}^{2} .
\end{gathered}
$$

Ao analisarmos as equações (3.65), (3.66) e (3.67), é possível extrair duas conclusões importantes. Primeiramente, a solução $\omega=0$ não é uma solução trivial, pois, para este caso, $\tilde{v}_{\|}=-2 \tilde{\omega}_{E} \cos \theta / k_{\|} \neq 0$. Conforme explicado mais adiante, esta solução corresponde aos fluxos zonais. A incompressibilidade do plasma, de acordo com (3.66), bem como a ausência de correntes diamagnéticas, pois $\tilde{p}=0$, é uma característica fundamental destes fluxos estacionários. A segunda característica importante é com relação ao fator de segurança. Note que para $q \rightarrow \infty$, $\tilde{v}_{\|} \rightarrow 0$ no caso de GAM $(\omega \neq 0)$ e $\tilde{v}_{\|} \rightarrow \infty$ para $\mathrm{ZF}$, pois $k_{\|}=1 / q R_{0}$.

É interessante observar também o que ocorre se $\tilde{\omega}_{E}=0$, ou seja, na ausência do campo elétrico. De acordo com a equação (3.62), a divergência da velocidade é proporcional à variação da velocidade paralela com relação ao ângulo poloidal $(\theta)$, o que induz uma perturbação na pressão, de acordo com (3.63). Adotando o mesmo procedimento, obtém-se uma equação similar à eq. (3.64),

$$
\left(1-\frac{k_{\|}^{2} c_{s}^{2}}{\omega^{2}}\right) \tilde{v}_{\|}=0
$$

que possui duas soluções. A primeira, trivial, $\tilde{v}_{\|}=\tilde{\rho}=\tilde{p}=0$ e portanto não importante e a segunda, $\omega^{2}=k_{\|}^{2} c_{s}^{2}$, que corresponde a ondas acústicas. Note que a segunda solução não permite a determinação das perturbações $\left(\tilde{v}_{\|}, \tilde{\rho}\right.$ e $\left.\tilde{p}\right)$ neste modelo simples. 
A corrente perturbada é composta por duas partes fundamentais para estes modos, a contribuição inercial e diamagnética, cujas expressões analíticas para suas componentes radiais são:

$$
\begin{gathered}
\tilde{j}_{\mathrm{I} r}=\left(\frac{\rho \mathbf{B}}{B^{2}} \times \frac{\partial \tilde{\mathbf{v}}}{\partial t}\right) \cdot \hat{\mathbf{e}}_{r} \approx i \frac{R_{0}}{B_{0}} \rho_{0} \omega \tilde{\omega}_{E}, \\
\tilde{j}_{\mathrm{p} r}=\left(\frac{\mathbf{B}}{B^{2}} \times \nabla \tilde{p}\right) \cdot \hat{\mathbf{e}}_{r} \approx \frac{-1}{\varepsilon B_{0} R_{0}} \frac{\partial \tilde{p}}{\partial \theta}(1+\varepsilon \cos \theta),
\end{gathered}
$$

Note que em (3.70) mantivemos o termo $\varepsilon \cos \theta$, que é proveniente de $B \approx B_{0}(1-\varepsilon \cos \theta)$, pois este termo é relevante no cálculo da média em uma superfície magnética.

A partir do desenvolvimento de (E.73) resulta a relação de dispersão:

$$
D=-i \frac{2 R_{0} \rho_{0}}{r B_{0}}\left(1+\frac{i \tilde{p}_{s}}{\rho_{0} \omega \tilde{\omega}_{E} R_{0}^{2}}\right) \omega \tilde{\omega}_{E}=\tilde{K} D^{(0)}=0
$$

onde $\tilde{K}=-2 i R_{0} \rho_{0} \tilde{\omega}_{E} / r B_{0}$ é um termo importante no estudo de auto-modos. No contínuo, a equação

$$
D^{(0)}=\omega\left[\frac{\omega^{2}-\left(2 c_{s}^{2} / R_{0}^{2}+k_{\|}^{2} c_{s}^{2}\right)}{\omega^{2}-k_{\|}^{2} c_{s}^{2}}\right]=0,
$$

fornece as soluções para as frequências dos ZF e GAM,

$$
\omega_{\mathrm{ZF}}=0, \quad \omega_{\mathrm{GAM}}^{2}=\left(2+\frac{1}{q^{2}}\right) \frac{c_{s}^{2}}{R_{0}^{2}}
$$

Na realidade, para ZF, como não foram considerados termos de ordem superior, em princípio, a solução é melhor descrita por $\omega_{\mathrm{ZF}} \approx 0$.

Note que, em ordem dominante, há também uma componente poloidal da corrente diamagnética, cuja expressão é

$$
\tilde{j}_{\mathrm{p} \theta}=\frac{i \mathrm{k}_{r} \tilde{p}}{B_{0}}
$$


Utilizando (D.19) e considerando $\mathrm{k}_{r} \gg r^{-1}$, obtemos uma relação de dispersão como forma alternativa à eq. (3.71):

$$
\nabla \cdot \tilde{\mathbf{j}} \approx i \mathrm{k}_{r} \tilde{j}_{\mathrm{I} r}-2 \tilde{j}_{\mathrm{p} \theta} \frac{\sin \theta}{R_{0}}+k_{\|} \frac{\partial \tilde{j}_{\|}}{\partial \theta}=0
$$

que, quando desenvolvida algebricamente, resulta em

$$
-\frac{\rho_{0} R_{0} \mathrm{k}_{r} \tilde{\omega}_{E}}{B_{0}} \omega\left(1-\frac{2 c_{s}^{2} / R_{0}^{2}}{\omega^{2}-k_{\|}^{2} c_{s}^{2}}\right)-\frac{\rho_{0} R_{0} \mathrm{k}_{r} \tilde{\omega}_{E}}{B_{0}} \frac{2 \omega c_{s}^{2} / R_{0}^{2}}{\omega^{2}-k_{\|}^{2} c_{s}^{2}} \cos (2 \theta)+k_{\|} \frac{\partial \tilde{j}_{\|}}{\partial \theta}=0 .
$$

Como para qualquer $\theta$ a equação (3.76) deve ser satisfeita, o termo contido no primeiro parênteses dessa equação deve se anular, resultando, assim, nas soluções mostradas na equação (3.73). Uma vantagem do uso de (3.76) é a de obtenção da corrente paralela,

$$
\tilde{j}_{\|}^{(\mathrm{GAM})}=\frac{\sqrt{2 q^{2}+1}}{4} \frac{\rho_{0} R_{0}}{B_{0}} \mathrm{k}_{r} \tilde{\omega}_{E} \sin (2 \theta), \quad \tilde{j}_{\|}^{(\mathrm{ZF})}=0,
$$

a qual se mostra dependente de segundos harmônicos, representados pelo termo $\sin (2 \theta)$. Note que, principalmente no limite $q \gg 1$, a contribuição da corrente paralela, $\tilde{j}_{\|} \propto q$, é significativa, justificando, em princípio considerar efeitos eletromagnéticos, pois $\tilde{j}_{\|}=\mathbf{b} \cdot \boldsymbol{\nabla} \times \tilde{\mathbf{B}}$. Além disso, em muitos experimentos GAM são detectados através da análise de segundas harmônicas $[25,80]$, de forma que a corrente paralela perturbada desempenha um papel importante neste tipo de oscilação.

A seguir, uma descrição simplificada do mecanismo físico envolvido nas oscilações presentes nos GAM é apresentada. Para simplificar as expressões e o raciocínio lógico deste mecanismo, consideramos o limite $q \rightarrow \infty$, ou seja, $\omega_{G A M}=\sqrt{2} c_{s} / R_{0}$. Supomos que, inicialmente, em $t=0$, exista um campo elétrico máximo, que é da forma $\tilde{\boldsymbol{E}}=\tilde{\omega}_{E} B_{0} R_{0} \hat{\mathbf{e}}_{r}$, onde $\tilde{\omega}_{E}=\left|\tilde{\omega}_{E}\right| \cos (\omega t)$,

$$
\left|\tilde{\omega}_{E}\right|=\frac{1}{2} \frac{e\left|\tilde{\Phi}_{0}\right|}{T_{i}} \mathrm{k}_{r} \rho_{i} \omega_{i}, \quad \tilde{\Phi}_{0}=\tilde{\Phi}_{0}(r, t)
$$

e consideramos $\mathrm{k}_{r} \rho_{i}>0$ por simplicidade ${ }^{1}$. As partículas do plasma, influenciadas por este

\footnotetext{
${ }^{1}$ Nada impede que $\mathrm{k}_{r} \rho_{i}<0$, pois a dependência radial de $\tilde{\Phi}$ e, consequentemente, de sua derivada radial, são desconhecidas em princípio.
} 
campo elétrico, bem como pelo campo magnético toroidal de equilíbrio, $B \approx B_{0}(1-\varepsilon \cos \theta)$, sofrem um movimento de deriva do tipo $\boldsymbol{E} \times \mathbf{B}$, o que produz um fluxo poloidal compressível, que é da forma

$$
\tilde{\mathbf{v}}_{E}=\left|\tilde{\omega}_{E}\right| R_{0}(1+\varepsilon \cos \theta) \cos (\omega t) \hat{\mathbf{e}}_{\theta}, \quad \tilde{v}_{\|} \approx 0,
$$

ou seja, de intensidade diferente nos lados de campo forte e de campo fraco, $\mathrm{HFS}^{2}$ e $\mathrm{LFS}^{3}$, conforme ilustra a figura 3.2 (a). Em decorrência desta diferença de intensidade, o plasma é comprimido, na razão

$$
\boldsymbol{\nabla} \cdot \tilde{\mathbf{v}}=-2\left|\tilde{\omega}_{E}\right| \sin \theta \cos (\omega t)
$$

o que ocasiona uma perturbação na densidade e, consequentemente, na pressão,

$$
\tilde{p}=\sqrt{2}\left|\tilde{\omega}_{E}\right| \rho_{0} c_{s} R_{0} \sin \theta \sin (\omega t),
$$

Com o movimento de deriva $\boldsymbol{E} \times \mathbf{B}$ do plasma, surge uma corrente inercial, que é radial e aproximadamente constante, que tende a anular o campo elétrico inicial pelo transporte de carga positiva para fora da superfície magnética de referência. Entretanto, em decorrência do gradiente poloidal de pressão, causada pela perturbação desta, surge também, uma corrente diamagnética, que em determinadas posições supera fortemente a primeira. O tempo em que é máxima a amplitude da corrente radial total e a expressão analítica das correntes inercial e diamagnética são mostrados, respectivamente, na figura 3.2 (b) e na equação (3.82), abaixo:

$$
\tilde{j}_{\mathrm{I} r}=\sqrt{2} \frac{\rho_{0} c_{s}}{B_{0}}\left|\tilde{\omega}_{E}\right| \sin (\omega t), \quad \tilde{j}_{\mathrm{p} r}=-\tilde{j}_{\mathrm{I} r}\left(\frac{1}{2}+\frac{1}{\varepsilon} \cos \theta+\frac{1}{2} \cos (2 \theta)\right) .
$$

Em média, neste momento, é máximo o transporte de cargas positivas para fora da superfície magnética (em laranja na figura 3.2), o que anula o campo elétrico radial e, consequentemente

\footnotetext{
${ }^{2}$ High Field Side

${ }^{3}$ Low Field Side
} 


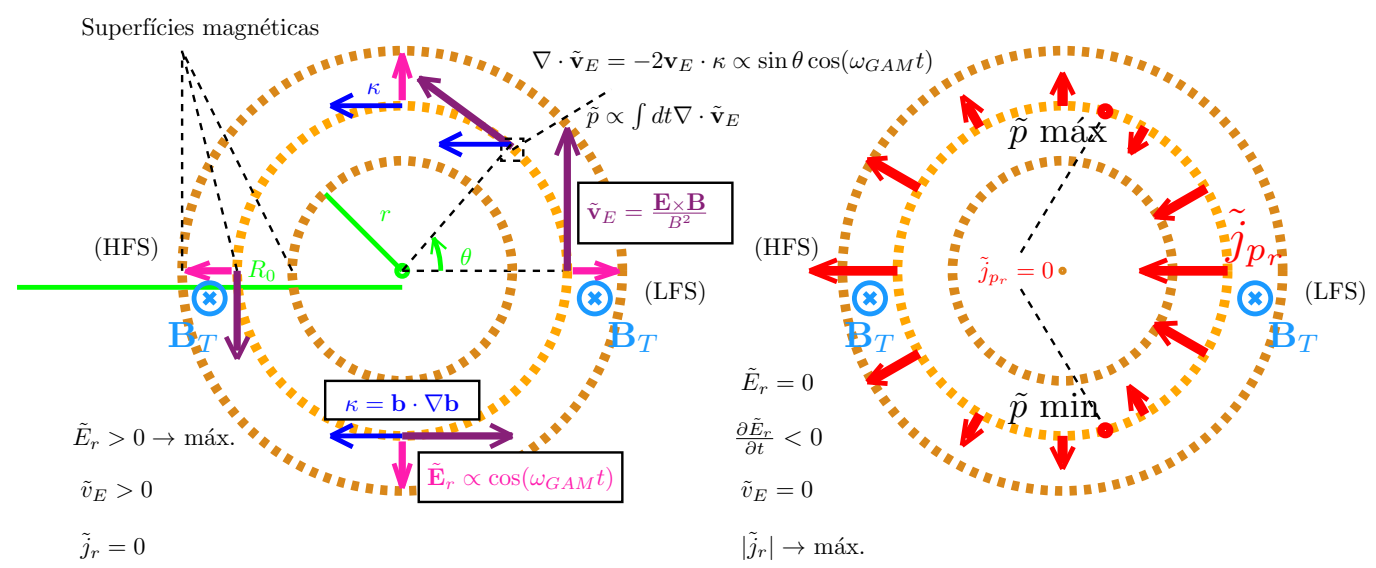
a) Instante inicial $t=0$
b) Instante $t=\pi / 2 \omega_{G A M}$

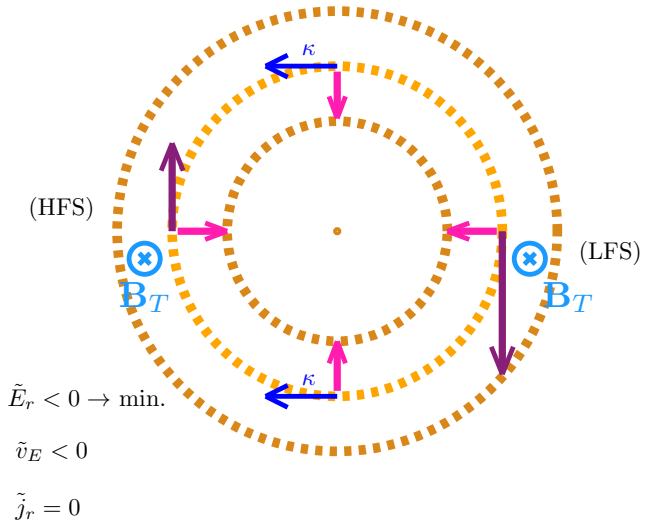

c) Instante $t=\pi / \omega_{G A M}$

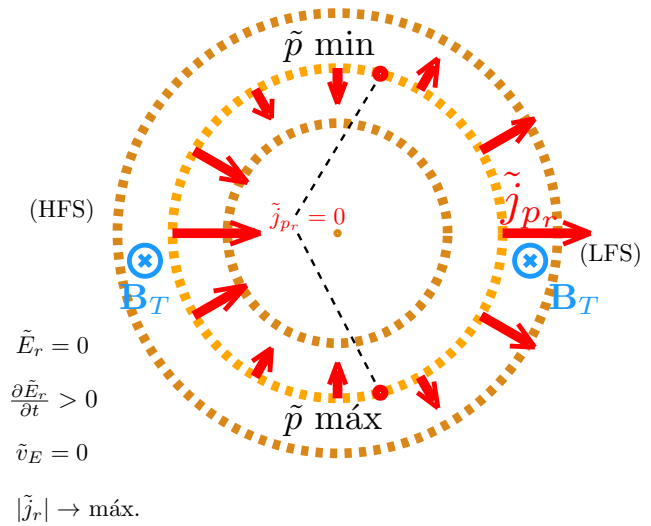

d) Instante $t=3 \pi / 2 \omega_{G A M}$

Figura 3.2: Dinâmica de modos acústicos geodésicos (GAM) em tokamaks.

a velocidade de deriva $\boldsymbol{E} \times \mathbf{B}$. Entretanto, devido a inércia de íons e à corrente diamagnética ainda presentes, o campo elétrico inverte seu sentido e, em $t=\pi / \omega_{G A M}$, a velocidade de deriva é 
máxima e no sentido anti-poloidal, conforme ilustra a figura 3.2 (c). Em $t=3 \pi / 2 \omega_{G A M}$ o campo elétrico é nulo novamente e a corrente é máxima, porém no sentido favorável ao transporte de carga positiva para a superfície magnética em questão, conforme a figura 3.2 (d). Finalmente, em $t=2 \pi / \omega_{G A M}$ a dinâmica descrita acima se repete. Uma investigação experimental tanto do valor da densidade perturbada como de sua posição poloidal de máximo valor absoluto é apresentada por A. Krämer-Fleken et. al. [81].

No caso dos ZF a dinâmica é consideravelmente mais simples. Ao se comportar de forma compressível, devido a um fluxo de retorno na direção paralela a $\mathbf{B}$,

$$
\tilde{v}_{\|}=-2 q \tilde{\omega}_{E} R_{0} \cos \theta, \quad \tilde{\omega}_{E}=\left|\tilde{\omega}_{E}\right|
$$

o plasma não permite perturbações da densidade (pressão) e, em consequência, apenas um fluxo estacionário poloidal e outro toroidal, normalmente de amplitude bem maior que o primeiro, podem coexistir. Normalmente a componente poloidal destes fluxos possui cisalhamento radial, invertendo de sentido com a posição radial em um intervalo espacial correspondente ao comprimento de onda radial. Este cisalhamento permite o controle de turbulência causada por ondas de deriva [11].

\subsection{Efeito de rotação nos GAM e ZF}

Pelo fato de o sistema (3.51)-(3.53) ser linear, podemos escrever as quantidades perturbadas como combinações das contribuições fundamentais $(0)$, toroidal $(\mathrm{T})$ e poloidal $(\mathrm{P})$, de acordo com a forma:

$$
\tilde{X}=\tilde{X}^{(0)}+\tilde{X}^{(T)}+\tilde{X}^{(P)}
$$

onde $\tilde{X}^{(0)}$ é a solução obtida quando $M_{P}=M_{T}=0, \tilde{X}^{(T)}$ é a contribuição toroidal (quando se considera apenas rotação toroidal) e o $\tilde{X}^{(P)}$ é a contribuição poloidal. Ressaltamos, no entanto, que, quando os dois tipos de rotação são considerados, nos termos $\Delta_{\rho}$ e $\Delta_{p}$ contidos em $\tilde{X}^{(T)}$ e $\tilde{X}^{(P)}$, é necessário considerar $M_{P} \neq 0$. 
Na parte restante desta seção, assim como no apêndice E, consideramos a normalização

$$
\Omega=\frac{\omega}{k_{\|} c_{s}}, \quad \tilde{\Omega}_{E}=\frac{\tilde{\omega}_{E}}{k_{\|} c_{s}}
$$

e, nesse apêndice, obtemos a relação de dispersão, a qual é mostrada a seguir:

$$
\frac{2 \tilde{\Omega}_{E}}{\Omega^{2}-1}\left(D^{(0)}+D^{(\mathrm{T})}+D^{(\mathrm{P})}\right)=0
$$

onde

$$
\begin{gathered}
D^{(0)}=\frac{\Omega}{2 q^{2}}\left(-\Omega^{2}+2 q^{2}+1\right), \\
D^{(\mathrm{T})}=\frac{M_{T}^{2}}{\Omega}\left[\left(1+\frac{1}{2} \frac{\Delta_{V}}{M_{T}}+\frac{1}{\gamma} \frac{\Delta_{p}}{M_{T}^{2}}+\frac{1}{2} \Delta_{\rho}\right) \Omega^{2}+\frac{1}{2}\left(\frac{\Delta_{p}}{\gamma}-\Delta_{\rho}\right)\right], \\
D^{(\mathrm{P})}=\frac{\mathcal{N}_{+1}^{p}{ }^{(\mathrm{P})}}{\mathcal{D}_{+1}{ }^{(\mathrm{P})}}-\frac{\mathcal{N}_{-1}^{p}{ }^{(\mathrm{P})}}{\mathcal{D}_{-1}(\mathrm{P})}+M_{T}\left[\frac{\mathcal{N}_{+1}^{v}(\mathrm{P})}{\mathcal{D}_{+1}(\mathrm{P})}-\frac{\mathcal{N}_{-1}^{v}(\mathrm{P})}{\mathcal{D}_{-1}^{(\mathrm{P})}}+\frac{M_{T}}{2}\left(\frac{\mathcal{N}_{+1}^{\rho}(\mathrm{P})}{\mathcal{D}_{+1}(\mathrm{P})}-\frac{\mathcal{N}_{-1}^{\rho}(\mathrm{P})}{\mathcal{D}_{-1}(\mathrm{P})}\right)\right], \\
\mathcal{D}_{ \pm 1}{ }^{(\mathrm{P})} \approx\left(M_{P} \mp \Omega\right)\left(\Omega+1 \mp M_{P}\right)\left(\Omega-1 \mp M_{P}\right)+\left[2 \gamma\left(\Omega \mp M_{P}\right)^{2}-1\right] M_{\mathrm{th}} .
\end{gathered}
$$

Antes de prosseguir com o desenvolvimento algébrico de (3.87)-(3.89), cujos extensos detalhes são apresentados no apêndice E, é conveniente calcular as singularidades em $D^{(\mathrm{P})}$ e, para isso, considera-se que $M_{\mathrm{th}} \sim M_{P}^{3}$ de forma a tornar possível, por meio de aproximações, resolver analiticamente $\mathcal{D}_{ \pm 1}{ }^{(\mathrm{P})}=0$. Os valores das singularidades, considerando $M_{P} \geq 0$, são mostrados graficamente na figura 3.3.

Finalmente, apresentamos a seguir a relação final proveniente do desenvolvimento algébrico de (3.87)-(3.89),

$$
D^{(0)}=\Omega\left(-\frac{\Omega^{2}}{2 q^{2}}+1+\frac{1}{2 q^{2}}\right),
$$




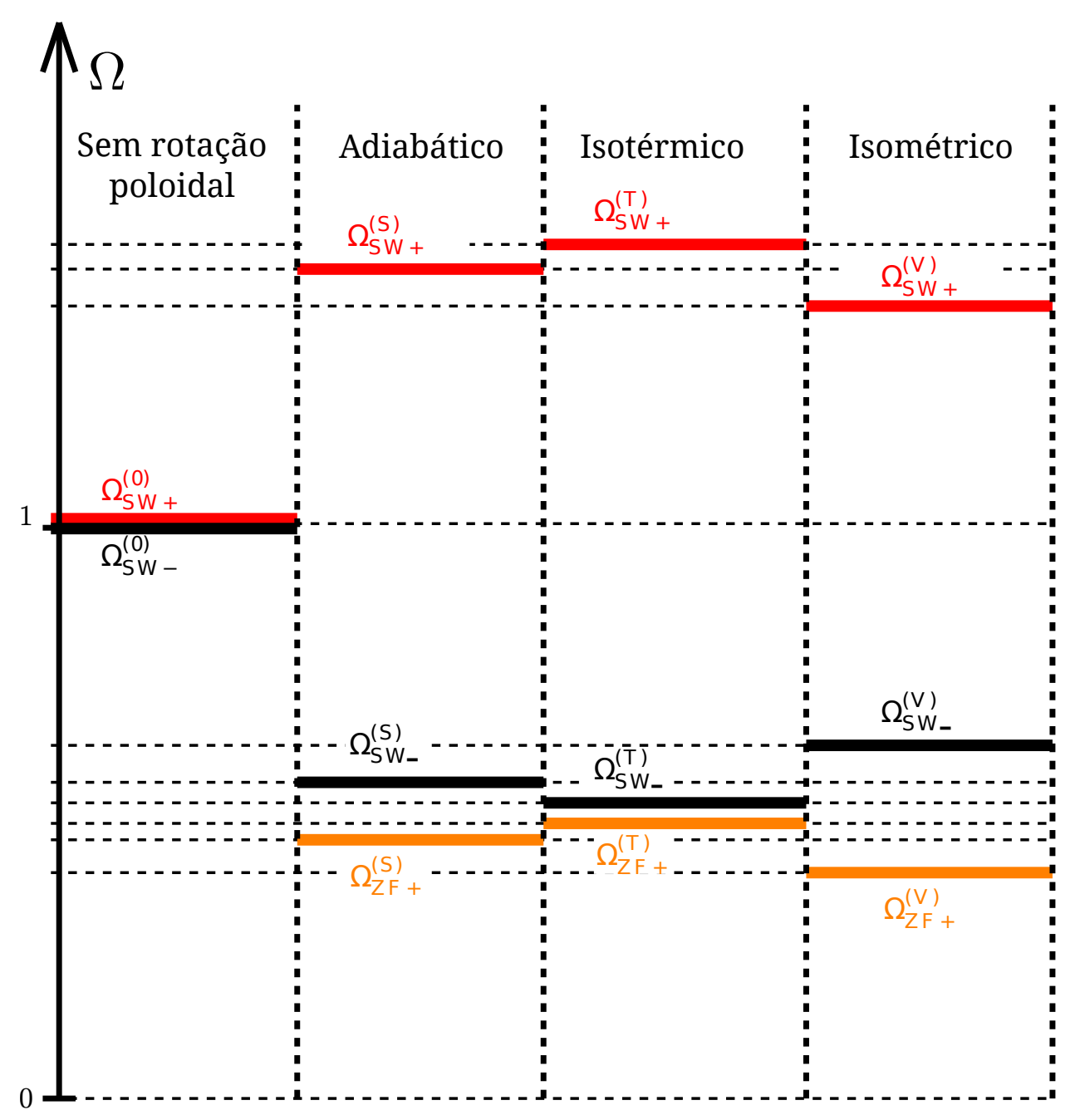

Figura 3.3: Singularidades do denomindador de $D^{(\mathrm{P})}$ para $M_{P} \geq 0$

$$
\begin{aligned}
& D^{(\mathrm{T})}=\frac{M_{T}^{2}}{\Omega}\left\{\left[2\left(1+M_{P}^{2}\right)\left(1-\frac{M_{P}}{M_{T}}+\frac{1}{2} \frac{M_{P}^{2}}{M_{T}^{2}}\right)+\left(\frac{1}{4}-\frac{M_{P}}{M_{T}}\right) M_{T}^{2}+\right.\right. \\
& \left.\left.\left(\frac{1}{2}-\frac{M_{P}}{M_{T}}+\frac{M_{P}^{2}}{M_{T}^{2}}\right) \frac{M_{\text {th }}}{M_{P}}\right] \Omega^{2}-\frac{1}{2} \frac{M_{\text {th }}}{M_{P}}\right\}
\end{aligned}
$$

onde os coeficientes $K_{2 k+1}=K_{2 k+1}\left(M_{P}, M_{T}, M_{\mathrm{th}}\right)$ são mostrados no apêndice E. A resolução analítica de (3.86), tendo em vista as expressões acimas, é efetuada mediante a seguinte aproximação assimptótica: 
- Ramo acústico geodésico (GAM): $\Omega \gg 1$.

- Ramo sonoro de íon (SW): $\Omega \sim 1$.

- Fluxos zonais(ZF): $\Omega \sim M_{P} \ll 1$.

No primeiro e no terceiro caso, o polinômio (3.93) têm seu grau reduzido quando o desenvolvemos em uma série de potência em $\Omega$ considerando apenas os três termos mais dominantes. O segundo caso pode ser analisado, ao assumirmos soluções da forma $\Omega^{2} \approx 1+\mathcal{O}\left(M_{P}^{2}\right)$, de modo que o denominador de (3.93) torna-se pequeno e, portanto, podemos considerar $D^{(\mathrm{P})} \approx 0$, obtendo, assim, a solução no ramo sonoro.

A seguir analisamos separadamente o caso com rotação apenas toroidal e o caso em que a rotação se desenvolve em ambas as direções.

\subsubsection{Efeito da rotação toroidal}

Com a substituição $M_{P}=0$ em (3.86) e (3.93) obtemos apenas duas soluções:

$$
\begin{gathered}
\frac{\omega_{G A M}^{2}}{c_{s}^{2} / R_{0}^{2}}=2+\frac{1}{q^{2}}+4 M_{T}^{2}+\left(2 q^{2} \frac{\Delta_{\rho}}{M_{T}^{2}}+\frac{1}{2}\right) \frac{M_{T}^{4}}{2 q^{2}+1}, \\
\frac{\omega_{Z F}^{2}}{c_{s}^{2} / R_{0}^{2}}=\left(\Delta_{\rho}-\frac{\Delta_{p}}{\gamma}\right) \frac{M_{T}^{2}}{2 q^{2}+1}, \quad \Delta_{p}=\gamma \frac{M_{T}^{2}}{2},
\end{gathered}
$$

que correspondem, respectivamente a GAM e a ZF. Na tabela 3.1, os valores das frequências relativas a estes modos são mostradas nos três regimes mais importantes: adiabático, isotérmico e isométrico.

\subsubsection{Efeito da rotação poloidal e toroidal}

A seguir consideramos os regimes adiabático e isotérmico na análise do efeito de rotação poloidal e toroidal nos GAM e ZF. No ramo geodésico e no ramo acústico de íons, as correspondentes frequências são comuns nestes dois regimes e, para $q \gg 1$, podem ser aproximadas 
Tabela 3.1: Comparação entre os quadrados das frequências normalizadas (por $c_{s} / R_{0}$ ) dos GAM e dos ZF nos regimes isométrico, adiabático e isotérmico.

\begin{tabular}{|l|l|c|}
\hline Regime & GAM $\left(R_{0}^{2} \omega_{G A M}^{2} / c_{s}^{2}\right)$ & $\mathbf{Z F}\left(R_{0}^{2} \omega_{Z F}^{2} / c_{s}^{2}\right)$ \\
\hline Isométrico & $2+\frac{1}{q^{2}}+4 M_{T}^{2}+\frac{M_{T}^{4}}{4 q^{2}+2}$ & $-\frac{M_{T}^{4}}{4 q^{2}+2}$ \\
\hline Adiabático & $2+\frac{1}{q^{2}}+4 M_{T}^{2}+\frac{M_{T}^{4}}{2}$ & 0 \\
\hline Isotérmico & $2+\frac{1}{q^{2}}+4 M_{T}^{2}+\left(2 \gamma q^{2}+1\right) \frac{M_{T}^{4}}{4 q^{2}+2}$ & $(\gamma-1) \frac{M_{T}^{4}}{4 q^{2}+2}$ \\
\hline
\end{tabular}

por:

$$
\begin{gathered}
\frac{\omega_{G A M}^{2}}{c_{s}^{2} / R_{0}^{2}} \approx 2+\frac{1}{q^{2}}+M_{P}^{2}+\left(M_{P}-2 M_{T}\right)^{2} \\
\frac{\omega_{S W}^{2}}{c_{s}^{2} / R_{0}^{2}} \approx \frac{1}{q^{2}}+\frac{\left(3 M_{P}-4 M_{T}\right)}{q^{2}} M_{P} .
\end{gathered}
$$

Em se tratando de $\mathrm{ZF}$, no regime adiabático a frequência não se altera ao contrário do que ocorre no regime isotérmico, no qual, devido ao efeito do fluxo de calor (q),

$$
\frac{\omega_{Z F}^{2}}{c_{s}^{2} / R_{0}^{2}} \approx \frac{M_{P}^{2}}{q^{2}}
$$

A expressão (3.98) é aproximada e válida apenas no limite $q \gg 1, M_{P}^{2} \ll 1$ e $M_{T}^{4} \ll M_{P}^{2}$.

Historicamente, os resultados mostrados em (3.96) e (3.97) nos GAM devido ao efeito de rotação poloidal foram obtidas primeiramente por V. I. Ilgisonis et. al. [50] considerando o regime adiabático. A partir do estudo deste trabalho, considerando o efeito de fluxo de calor, no regime isotérmico, obtivemos a correção dos fluxos zonais [76]. Há ainda o regime isométrico a ser analisado, o que pretendemos fazer em um trabalho futuro. 


\subsection{Discussão sobre o índice adiabático}

Antes do início da próxima seção, é conveniente expressar $\omega_{G A M}$ em termos da velocidade térmica de íons. Esta conveniência se deve ao intuito de comparar a teoria de um fluido com a teoria de dois fluidos. Desta forma, conforme (3.73), a frequência dos GAM pode ser expressa como:

$$
\omega_{G A M}^{2}=\left(2+\frac{1}{q^{2}}\right) \frac{\gamma p_{0}}{\rho_{0}}=\gamma\left(1+\frac{1}{2 q^{2}}\right)\left(1+\frac{T_{e}}{T_{i}}\right) \frac{v_{T_{i}}^{2}}{R_{0}^{2}}
$$

onde as relações: $p_{0} \approx n_{0}\left(T_{i}+T_{e}\right)$ e $\rho_{0} \approx n_{0} m_{i}$ foram utilizadas. A teoria de um fluido não considera a diferença entre os índices adiabáticos $(\gamma)$ de íons e de elétron. De fato, conforme a teoria cinética, a suposição mais realista para plasmas de tokamak é $\gamma_{i}=5 / 3 \approx 1,7$ e $\gamma_{e}=1$. Esta discrepância de valores se deve à grande diferença entre a massa de íons e de elétrons, de forma que, por apresentarem inércia muito menor os elétrons são capazes de rapidamente entrarem em equilíbrio térmico entre si. Desta forma, para efeitos de comparação entre as duas teorias, é conveniente utilizar a substituição:

$$
\gamma \rightarrow \gamma^{(\text {correto })}=\frac{\gamma_{i}+\gamma_{e} T_{e} / T_{i}}{1+T_{e} / T_{i}}
$$

onde, para $T_{e}=T_{i}, \gamma^{\text {(correto) }} \approx 1,3<5 / 3 \approx 1,7$, representando um erro de aproximadamente $25 \%$.

Na próxima seção, além de derivarmos uma relação mais precisa para a frequência dos GAM com relação ao índice adiabático, consideramos também o efeito da anisotropia de pressão, ou seja, $\tilde{p}_{\perp} \neq \tilde{p}_{\|}$. Este efeito resulta em um aumento do índice adiabático efetivo para íons $\left(\gamma_{i}=5 / 3 \rightarrow \gamma_{i}^{(\text {efetivo })}=7 / 4\right)$. Para simplificar o modelo, nos restringimos ao limite $q \rightarrow \infty$. Porém, no próximo capítulo, no qual tratamos a respeito da teoria cinética, consideramos correções de $\mathcal{O}\left(q^{-2}\right)$ na frequência dos GAM. 


\subsection{Modelo de dois fluidos com viscosidade paralela}

Nesta seção partimos do sistema (2.66)-(2.69) para descrever plasmas no qual efeitos de gradientes de densidade e de temperatura devem ser considerados, porém, não levamos em conta no equilíbrio rotação e nem fluxo de calor, de acordo com modelo apresentado em [36]. Desta forma, tal sistema é composto pelas seguintes equações:

$$
\begin{aligned}
& \frac{\partial \tilde{n}_{i}}{\partial t}+\nabla \cdot\left(n_{0} \tilde{\mathbf{v}}_{i}\right)=0 \\
& \frac{\partial \tilde{p}_{i}}{\partial t}+\tilde{\mathbf{v}}_{i} \cdot \boldsymbol{\nabla} p_{0_{i}}+\gamma p_{0_{i}} \boldsymbol{\nabla} \cdot \tilde{\mathbf{v}}_{i}=0 \\
& \frac{\partial \tilde{\pi}_{\|_{i}}}{\partial t}+p_{0_{i}}\left[-2 \tilde{\mathbf{v}}_{i} \cdot \nabla \ln B-\left(\gamma_{i}-1\right) \boldsymbol{\nabla} \cdot \tilde{\mathbf{v}}_{i}\right]=0, \\
& m_{i} n_{0} \frac{\partial \tilde{\mathbf{v}}_{i}}{\partial t}+\nabla \tilde{p}_{i}+\nabla \cdot \tilde{\boldsymbol{\pi}}_{\|_{i}}-e n_{0}\left(\tilde{\boldsymbol{E}}+\tilde{\mathbf{v}}_{i} \times \mathbf{B}\right)=0, \\
& m_{e} n_{0} \frac{\partial \tilde{\mathbf{v}}_{e}}{\partial t}+\nabla \tilde{p}_{e}+e n_{0}\left(\tilde{\boldsymbol{E}}+\tilde{\mathbf{v}}_{e} \times \mathbf{B}\right)=0 \\
& \nabla \cdot\left(\tilde{\mathbf{j}}_{i}+\tilde{\mathbf{j}}_{e}\right)=0
\end{aligned}
$$

A seguir focamos nos objetivos didáticos do presente modelo, de forma que inicialmente não levamos em conta efeitos de gradientes de densidade e de temperatura. Contudo, tais efeitos são considerados posteriormente, ainda neste capítulo. 


\subsubsection{Efeito de anisotropia de pressão nos GAM}

Inicialmente, a partir do desenvolvimento algébrico de (3.101) -(3.103) no limite $q \gg 1$, considerando $\tilde{\mathbf{v}}_{i} \approx \tilde{\mathbf{v}}_{E}$,

$$
\begin{gathered}
\frac{\partial \tilde{n}_{i}}{\partial t}-2 n_{0} \tilde{\mathbf{v}}_{E} \cdot \nabla \ln B=0, \\
\frac{\partial \tilde{p}_{i}}{\partial t}-2 \gamma_{i} p_{0_{i}} \tilde{\mathbf{v}}_{E} \cdot \nabla \ln B=0, \\
\frac{\partial \tilde{\pi}_{\| i}}{\partial t}-2\left(2-\gamma_{i}\right) p_{0_{i}} \tilde{\mathbf{v}}_{E} \cdot \nabla \ln B=0 .
\end{gathered}
$$

obtemos as seguintes relações:

$$
\tilde{n}_{i_{ \pm 1}}= \pm \frac{i}{2} \frac{\omega_{\mathrm{di}}}{\omega} \frac{e \tilde{\Phi}_{0}}{T_{i}} n_{0}, \quad \tilde{p}_{i}=\gamma_{i} T_{i} \tilde{n}_{i}, \quad \tilde{\pi}_{\| i}=\left(2-\gamma_{i}\right) T_{i} \tilde{n}_{i}
$$

Para elétrons, a dinâmica é consideravelmente diferente, pois estes, devido a sua pequena inércia, são considerados no regime adiabático e isotérmico. Desta forma, como $m_{e} \ll m_{i}$, a partir de (3.105), obtemos a componente paralela da equação de momento,

$$
\nabla_{\|} \tilde{p}_{e}+e n_{0} \tilde{E}_{\|}=0, \quad \tilde{E}_{\|}=-\nabla_{\|} \tilde{\Phi}
$$

que, quando utilizada em conjunto com equações similares a (3.107) e (3.108), porém para elétrons, fornece relações similares às obtidas em (3.110):

$$
\tilde{p}_{e}=T_{e} \tilde{n}_{e}, \quad \tilde{n}_{e_{ \pm 1}}=\frac{e n_{0}}{T_{e}} \tilde{\Phi}_{ \pm 1}, \quad \tilde{T}_{e}=0
$$

É importante ter em mente que, ao contrário de $\tilde{v}_{\| i}$, mesmo no limite $q \gg 1$, $\tilde{\mathbf{v}}_{e}$ não pode ser desprezado, porém informações sobre a velocidade paralela de íons e de elétrons serão consideradas apenas em trabalhos futuros. Na presente análise, é importante é observar que devido a fato de que $\gamma_{e}=1$, conforme (3.112), elétrons não contribuem para a anisotropia da pressão 
$\left(\tilde{\pi}_{\| e} \approx 0\right)$

Da condição de quasi-neutralidade, $e\left(\tilde{n}_{i}-\tilde{n}_{e}\right)=0$, obtemos

$$
\tilde{\Phi}_{ \pm 1}= \pm i \frac{\tau_{e}}{2} \frac{\omega_{\mathrm{di}}}{\omega} \tilde{\Phi}_{0}, \quad \tau_{e}=\frac{T_{e}}{T_{i}}
$$

ou, na forma trigonométrica,

$$
\tilde{\Phi}_{s}=\tau_{e}\left(\omega_{i} / \omega\right) \mathrm{k}_{r} \rho_{i} \tilde{\Phi}_{0}, \quad \tilde{\Phi}_{c}=0, \quad \omega_{i}=\frac{v_{T_{i}}}{R_{0}}
$$

Note que em (3.114) utilizamos a substituição $\omega_{\mathrm{di}}=\mathrm{k}_{r} \rho_{i} \omega_{i}$, a qual tem por intuito mostrar que $\tilde{\Phi}_{s} \sim \mathrm{k}_{r} \rho_{i} \tilde{\Phi}_{0}$, onde, ao longo desta tese, consideramos $\mathrm{k}_{r} \rho_{i} \ll 1$.

A partir de (3.104) e (3.105), conforme mostrado anteriormente, obtém-se a densidade de corrente:

$$
\tilde{\mathbf{j}}_{\perp \alpha}=\tilde{\mathbf{j}}_{\mathrm{I} \alpha}+\tilde{\mathbf{j}}_{p_{\alpha}}+\tilde{\mathbf{j}}_{\pi_{\alpha}}+\tilde{\mathbf{j}}_{E \alpha}
$$

onde

$$
\tilde{\mathbf{j}}_{I i}=\frac{m_{i} n_{0}}{B} \mathbf{b} \times \frac{d \tilde{\mathbf{v}}_{E}}{d t}, \quad \tilde{\mathbf{j}}_{p_{\alpha}}=\frac{\mathbf{b} \times \nabla \tilde{p}_{\alpha}}{B}, \quad \alpha=i, e, \quad \tilde{\mathbf{j}}_{\pi_{i}}=\frac{\mathbf{b} \times \boldsymbol{\nabla} \cdot \tilde{\boldsymbol{\pi}}_{i}}{B}
$$

são as contribuições importantes que devem ser calculadas para a obtenção da relação de dispersão. Note que referente ao movimento de deriva $\boldsymbol{E} \times \mathbf{B}$, há um cancelamento, pois $\tilde{\mathbf{j}}_{E_{i}}+\tilde{\mathbf{j}}_{E_{e}}=0$, com relação aos elétrons, a contribuição da corrente inercial é pequena, ou seja, $\mathbf{j}_{\mathrm{I}_{e}}=\left(m_{e} / m_{i}\right) \mathbf{j}_{\mathrm{I}_{i}}$, podendo ser desprezada e, também, $\mathbf{j}_{\pi_{e}} \approx 0$. Desta forma, apenas as contribuições mencionadas em (3.116) são importantes para o cálculo da densidade de corrente total,

$$
\tilde{\mathbf{j}}_{\perp}=\sum_{\alpha=i, e} \tilde{\mathbf{j}}_{\perp \alpha} .
$$

Ao procedermos de forma similar ao procedimento adotado na seção 3.3, a partir da eq. 
(E.73), obtemos a relação de dispersão,

$$
\frac{e \tilde{\Phi}_{0}}{T_{i}} \mathrm{k}_{r} \rho_{i} \omega+\left(\frac{\tilde{p}_{i_{s}}}{n_{0} T_{i}}+\frac{\tilde{p}_{e_{s}}}{n_{0} T_{i}}+\frac{1}{4} \frac{\tilde{\pi}_{\| i_{s}}}{n_{0} T_{i}}\right) \omega_{i}=0
$$

cujo desenvolvimento é proveniente dos seguintes resultados:

$$
J_{I r}=-\frac{\mathrm{k}_{r} \rho_{i}^{2}}{2} \frac{e \tilde{\Phi}_{0}}{T_{i}} e n_{0} \omega, \quad J_{p r}+J_{\tilde{\pi}_{\|} r}=-\frac{\rho_{i}}{2} \frac{\omega_{i}}{\varepsilon} \frac{e}{T_{i}}\left[\frac{\partial}{\partial \theta}\left(\tilde{p}-\frac{\tilde{\pi}_{\|}}{2}\right)+3 \varepsilon \tilde{\pi}_{\|} \sin \theta\right] .
$$

Finalmente, com a substituição de (3.110), (3.112) e (3.113) em (3.118), obtemos a relação

$$
\mathrm{k}_{r}^{2} \rho_{i}^{2}\left[\omega-\frac{\omega_{i}^{2}}{\omega}\left(\gamma_{i}+\gamma_{e} \tau_{e}+\frac{2-\gamma_{i}}{4}\right)\right] \frac{e \tilde{\Phi}_{0}}{T_{i}}=0
$$

e, a partir desta, a frequência dos GAM,

$$
\frac{\omega_{\mathrm{GAM}}^{2}}{v_{T_{i}}^{2} / R_{0}^{2}}=\gamma_{i}+\gamma_{e} \tau_{e}+\frac{2-\gamma_{i}}{4}=\gamma_{i}^{(\mathrm{eff})}+\gamma_{e} \tau_{e}
$$

onde $\gamma_{i}^{\text {(eff) }}=3 \gamma_{i} / 4+1 / 2$ é o índice adiabático efetivo para íons.

Considerando $\gamma_{i}=5 / 3$ (íons no regime de fluido) e $\gamma_{e}=1$ (elétrons no regime adiabático e isotérmico), segue que $\gamma_{i}^{\text {(eff) }}=7 / 4$ e, consequentemente, segue que

$$
\omega_{\mathrm{GAM}}=\left(\frac{7}{4}+\frac{T_{e}}{T_{i}}\right)^{1 / 2} \frac{v_{T_{i}}}{R_{0}}
$$

conforme observado anteriormente [36]. Observa-se que o efeito da anisotropia da pressão de íons, presente no termo $\tilde{\pi}_{\| i}$, representa, teoricamente, um pequeno aumento na frequência dos GAM. Este aumento é de aproximadamente $3,0 \%$ para $\tau_{e}=1 \mathrm{e}$, para $\tau_{e} \gg 1$, o efeito é ainda menor (próximo de $1,7 \%$, considerando $\gamma=\gamma^{\text {(correto) }}$, conforme (3.100).

\subsubsection{Efeitos diamagnéticos nos GAM}

A seguir consideramos efeitos diamagnéticos (ou efeitos de deriva) nos modos GAM. Para simplificar as expressões, consideramos desde o início as substituições $\gamma_{i}=5 / 3$ e $\gamma_{e}=1$. Efeitos 
de deriva são provenientes de termos tais como $\tilde{\mathbf{v}}_{E} \cdot \nabla n_{0}$ e $\tilde{\mathbf{v}}_{E} \cdot \nabla T_{i 0}$, ou seja, ocorrem devido a gradientes radiais de densidade e temperatura de equilíbrio. Se comparadas com as eqs. (3.107) e (3.108), as equações a serem resolvidas neste caso, agora, apresentam termos adicionais:

$$
\begin{gathered}
\frac{\partial \tilde{n}_{i}}{\partial t}-2 n_{0} \tilde{\mathbf{v}}_{E} \cdot \nabla \ln B+\tilde{\mathbf{v}}_{E} \cdot \nabla n_{0}=0, \\
\frac{3}{2} \frac{\partial \tilde{p}_{i}}{\partial t}-5 p_{0_{i}} \tilde{\mathbf{v}}_{E} \cdot \nabla \ln B+\frac{3}{2} \tilde{\mathbf{v}}_{E} \cdot \nabla p_{0_{i}}=0,
\end{gathered}
$$

o que não ocorre com a eq. de evolução da viscosidade paralela, que permanece inalterada.

A solução para a densidade e pressão perturbadas de íons, neste caso,

$$
\begin{gathered}
\tilde{n}_{i_{ \pm 1}}=\left( \pm \frac{i}{2} \frac{\omega_{\mathrm{di}}}{\omega} \tilde{\Phi}_{0} \mp \frac{\omega_{* i}}{\omega} \tilde{\Phi}_{ \pm 1}\right) \frac{e n_{0}}{T_{i}} \\
\tilde{p}_{i_{ \pm 1}}=\left( \pm \frac{5}{3} \frac{i}{2} \frac{\omega_{\mathrm{di}}}{\omega} \tilde{\Phi}_{0} \mp\left(1+\eta_{i}\right) \frac{\omega_{* i}}{\omega} \tilde{\Phi}_{ \pm 1}\right) e n_{0}
\end{gathered}
$$

podem ser contrastadas com os resultados apresentados em (3.110), onde observa-se que os termos adicionais em (3.125) são provenientes de gradientes de densidade e de temperatura de equilíbrio. Os termos definidos como $\omega_{* i}=T_{i} / \operatorname{er} B L_{N}$ e $\omega_{* e}=T_{e} / \operatorname{er} B L_{N}$, onde $L_{N}^{-1}=d n_{0} / d r$ são conhecidos como frequências de deriva de íons e de elétrons, respectivamente. Também é comum encontrar, na literatura da área, a frequência diamagnética, que, no caso de íons, é definida como $\omega_{p i}^{*}=\left(1+\eta_{i}\right) \omega_{* i}$, onde $\eta_{i}=L_{N} / L_{T_{i}}$ e $L_{T_{i}}^{-1}=d T_{i} / d r$.

A dinâmica de elétrons não se altera pela presença de efeitos diamagnéticos eletrostáticos, porém, quando consideramos efeitos eletromagnéticos, conforme discutido em 3.8, o gradiente da temperatura de elétrons desempenha um papel fundamental nesta dinâmica.

Novamente, consideramos a condição de quasi-neutralidade, $\tilde{n}_{i}=\tilde{n}_{e}$, para obter a relação entre os harmônicos do potêncial eletrostático:

$$
\tilde{\Phi}_{ \pm 1}= \pm \frac{i}{2} \frac{\tau_{e} \omega_{\mathrm{di}}}{\omega \pm \omega_{* e}} \tilde{\Phi}_{0}
$$

de forma que, na presença de efeitos diamagnéticos, as componentes seno e cosseno (não nula 
na presença de efeitos diamagnéticos) do potencial eletrostático são dadas por

$$
\tilde{\Phi}_{s}=\frac{\tau_{e} \omega_{i} \omega}{\omega^{2}-\omega_{* e}^{2}} \mathrm{k}_{r} \rho_{i} \tilde{\Phi}_{0}, \quad \tilde{\Phi}_{c}=-i \frac{\tau_{e} \omega_{i} \omega_{* e}}{\omega^{2}-\omega_{* e}^{2}} \mathrm{k}_{r} \rho_{i} \tilde{\Phi}_{0}=-i \frac{\omega_{* e}}{\omega} \tilde{\Phi}_{s}
$$

Analogamente ao caso anterior, sem efeito diamagnético, o termo principal para o desenvolvimento algébrio é a componente $\sin \theta$ da quantidade $\tilde{p}+\tilde{\pi}_{\|} / 4$, cujo cálculo fornece

$$
\left(\tilde{p}+\frac{\tilde{\pi}_{\|}}{4}\right)_{s}=-\frac{\omega_{\mathrm{di}}}{\omega}\left(\frac{7}{4}+\frac{\tau_{e} \omega^{2}+\left(1+\eta_{i}\right) \omega_{* e}^{2}}{\omega^{2}-\omega_{* e}^{2}}\right) e n_{0} \tilde{\Phi}_{0}
$$

Conforme o procedimento anteriormente apresentado, o cálculo da média em uma superfície magnética das componente radial da densidade de corrente inercial e diamagnética fornece a relação de dispersão, que é uma equação quadrática em $\omega^{2}$ com soluções:

$$
\omega_{\mathrm{GAM} \pm}^{2}=\frac{1}{2}\left(\omega_{\mathrm{GAM}}^{2}+\omega_{* e}^{2} \pm \sqrt{\left(\omega_{\mathrm{GAM}}^{2}+\omega_{* e}^{2}\right)^{2}+\left(4 \eta_{i}-3\right) \omega_{* e}^{2} \omega_{i}^{2}}\right)
$$

onde $\omega_{\mathrm{GAM}}^{2}=\left(7 / 4+\tau_{e}\right) \omega_{i}^{2}$, da mesma forma como definido anteriormente.

Estas soluções que obtivemos, as quais foram publicadas em [77], podem ter suas expressões simplificadas se aproximadas no limite $\omega_{* e} \ll \omega_{i}$ :

$$
\omega_{\mathrm{GAM}+}^{2}=\omega_{\mathrm{GAM}}^{2}+\frac{1+\tau_{e}+\eta_{i}}{7 / 4+\tau_{e}} \omega_{* e}^{2} \quad \text { e } \quad \omega_{\mathrm{GAM}-}^{2}=\frac{3 / 4-\eta_{i}}{7 / 4+\tau_{e}} \omega_{* e}^{2}
$$

Observa-se que gradientes de densidade e temperatura causam um aumento na frequência dos GAM, que é proporcional à frequência de deriva de elétrons. Para $\eta_{i}=0,75$, a segunda solução (-) possui frequência próxima à dos ZF, o que pode desempenhar um papel importante na dinâmica que governa a turbulência de ondas de deriva devido à interação não linear entre estas duas frequências. Quando $\eta_{i}>0,75$, este modelo prevê uma instabilidade. É possível concluir que há claras indicações de que gradientes de temperatura iônica tendem a desestabilizar o plasma ao passo que gradientes de densidade contribuem para estabilizá-lo, de acordo com a análise do valor de $\eta_{i}$ na solução negativa de (3.130). 


\subsection{Discussão sobre GAM eletromagnético}

Com o intuito de apresentar opções para aprimoramento dos modelos para os GAM, discutimos a seguir o efeito causado pelo campo magnético perturbado perpendicular ao campo magnético de equilíbrio. Tais efeitos são descritos pelo potencial vetor paralelo, $\tilde{A}_{\|}$, de forma que os campos elétrico e magnético perturbados são dado por

$$
\tilde{\boldsymbol{E}}=-\nabla \tilde{\Phi}-\frac{\partial \tilde{A}_{\|}}{\partial t}, \quad \tilde{\mathbf{B}}=\boldsymbol{\nabla} \times\left(\tilde{A}_{\|} \mathbf{b}\right)
$$

A densidade de corrente paralela pode ser relacionada com o potencial vetor por meio do uso da lei de Àmpere,

$$
(\boldsymbol{\nabla} \times \tilde{\mathbf{B}}) \cdot \mathbf{b}=\mu_{0} \tilde{J}_{\|} \Longrightarrow \tilde{J}_{\|}=\frac{\mathrm{k}_{r}^{2}}{\mu_{0}} \tilde{A}_{\|}
$$

onde utilizamos as relações (D.34) e (D.36). Também é útil relacionar esta densidade com a velocidade, ou seja,

$$
\tilde{J}_{\|}=\tilde{J}_{\| i}+\tilde{J}_{\| e}, \quad \tilde{J}_{\| \alpha}=e_{\alpha} n_{0} \tilde{v}_{\| \alpha}
$$

de forma que é necessário determinar a componente paralela da velocidade de íons e de elétrons para relacionar $\tilde{A}_{\|} \operatorname{com} \tilde{\Phi}$.

A pressão e, consequentemente, a densidade de elétrons, são obtidas a partir da componente paralela da equação de momento, (3.104), porém, é necessário considerar a contribuição de $\tilde{\mathbf{B}}$ neste cálculo. A equação resultante, então, fica,

$$
\nabla_{\|} \tilde{p}_{e}+\tilde{\nabla}_{\|} p_{e 0}+e n_{0} \tilde{E}_{\|}=0
$$

onde $\tilde{\nabla}_{\|}=(\tilde{\mathbf{B}} / B) \cdot \nabla$ é um operador cuja expressão é mostrada em (D.35) no apêndice D.4

e $\tilde{E}_{\|}=-\nabla_{\|} \tilde{\Phi}+i \omega \tilde{A}_{\|}$é a componente paralela do campo elétrico mostrado em (3.131). Note que, devido ao segundo termo de (3.134), que só esta presente no caso eletromagnético (conf. eq. (3.111)), surge $\eta_{e}=L_{N} / L_{T_{e}}$ e, portanto, é provável que a dinâmica de elétrons desempenhe 
um papel importante no caso eletromagnético.

Com relação a dinâmica de íons, ficam inalteradas as grandezas $\tilde{\pi}_{\| i}$ e $\tilde{p}_{i}$ com relação a $\tilde{n}_{i}$ calculadas anteriormente. Entretanto no caso magnético é necessário considerar as velocidades paralelas de elétrons e de íons nas suas respectivas equações da continuidade, (3.101)

Com o prosseguimento dos cálculos provenientes das equações e condições descritas acima, surgirá o importante termo $K_{\perp}^{2}=k_{\|}^{2} \mathrm{k}_{r}^{2} \lambda_{D_{e}}^{2} c^{2} / \omega^{2}$ adimensional, onde $\lambda_{D_{e}}=\sqrt{\varepsilon_{0} T_{e} / n_{0} e^{2}}$ é o comprimento de Debye para elétrons. O limite puramente eletrostático é obtido considerando $K_{\perp} \rightarrow \infty$, porém, por outro lado, quando $K_{\perp}<1$ efeitos eletromagnéticos passam a ser importantes da dinâmica dos GAM. Esta questão é discutida de forma mais geral em [82], onde o parâmetro $K_{\perp}$ foi definido. Em [68], partindo da equação cinética de deriva, é mostrado que o modo poloidal $m=2$ é importante no estudo de efeitos eletromagnéticos nos GAM.

\subsection{Sumário e discussão}

Neste capítulo, a partir da teoria da MHD ideal e do modelo de dois fluidos, quando a anisotropia da pressão perturbada de íons é considerada, obtivemos expressões analíticas para três importantes ramos de baixas frequências: fluxos zonais, acústico de íons e acústico geodésico. A distinção da ordem de grandeza das frequências pertencentes a estes ramos pode ter aplicações importantes se comparadas as expressões analíticas com valores experimentais das respectivas frequências. Ao passo que algumas aplicações possuem objetivos diagnósticos, tais como obter o perfil radial do fator de segurança, $q(r)$, e da temperatura, $T(r)$, outras se direcionam para a análise da estabilidade de fluxos zonais e modos acústicos. Identificar as condições em que ocorrem instabilidades pode ajudar a evitar a degradação do confinamento causado pelo transporte anômalo. Fluxos zonais, modos sonoros e modos acústicos (ZF, SW e GAM) são capazes de reduzir a turbulência causada por ondas de deriva por meio de um processo de auto-organização que ocorre no plasma, o qual ainda não é muito bem compreendido $[6,11]$ mas possui um forte impacto na área de controle de difusão de energia e partículas e, portanto, obter a frequência destes modos e as condições de instabilidade quando outros efeitos do plasma são considerados é importante. 
Inicialmente, partindo das equações da MHD ideal, investigamos o equilíbrio com rotação poloidal e toroidal. Nesta investigação constatamos que o gradiente radial de temperatura e, consequentemente, o fluxo de calor de equilíbrio por ele causado, estão relacionados à rotação poloidal. Entretanto, no regime adiabático, no qual não há fluxo de calor localmente, é possível encontrar um equilíbrio com rotação poloidal não nula. A inversão de sentido do gradiente de temperatura do plasma (tal situação é possível apenas localmente, pois a temperatura é maior no centro do que na borda) indicaria que houve uma mudança de regime, do regime adiabático para o isométrico, o que poderia causar instabilidade nos ZF. O detalhamento deste estudo, que se iniciou pelo trabalho de V. P. Lakhin [27], é uma das propostas para trabalhos futuros. Próximo à região $r=0.7 a$, observamos que o gradiente radial de temperatura é proporcional ao cubo da velocidade poloidal $\left(M_{\mathrm{th}} \propto M_{P}^{3}\right)$. Utilizando o modelo da MHD ideal e a teoria de dois fluidos no equilíbrio, é possível, pelo menos de forma aproximada, obter o perfil radial da temperatura de íons, o que do ponto de vista experimental é complicado de ser feito. Para isso é necessário ter informações sobre o perfil radial da velocidade de rotação poloidal e toroidal, o qual foi obtido experimentalmente para o tokamak TCABR $[78,79]$.

Para a obtenção das frequências decorrentes de perturbações eletrostática, desenvolvemos um método iterativo para um equilíbrio de regime arbitrário. Este método é desenvolvido em três etapas consecutivas baseadas nas seguintes condições de equilíbrio: $\operatorname{Sem} \operatorname{rotação~}\left(M_{P}=M_{T}=0\right)$, com rotação unicamente toroidal $\left(M_{P}=0, M_{T} \neq 0\right)$ e, finalmente, com rotação poloidal e toroidal $\left(M_{P} \neq 0, M_{T} \neq 0\right)$. Este método é justificável pelo fato de que o sistema a ser resolvido é linear e, portanto, o princípio da superposição pode ser aplicado. A motivação para este método é proveniente do estudo realizado por G. N. Throumoulopoulos [83], a respeito da inexistência de equilíbrio com rotação unicamente poloidal. Com relação a este tema, há ainda questões em aberto, pois, de acordo com a análise da figura 3.1, observa-se que em $r \approx 0.7 a$ o valor da velocidade poloidal é próximo do máximo e a velocidade toroidal se anula nesta posição. Neste região também ocorre a inversão de sentido da rotação toroidal, o que ainda não é bem compreendida do ponto de vista teórico mas pode ter um forte impacto na formação da barreira de transporte [10] e, consequentemente, no transporte turbulento. No centro da coluna de plasma a resistividade é muito baixa e o plasma pode ser considerado não-colisional, porém, conforme nos 
aproximamos da borda da coluna de plasma, este se torna colisional e, portanto, o estudo nesta região requer, em princípio, um modelo de fluido mais abrangente, capaz de incluir viscosidade, resistividade e contribuições colisionais para o fluxo de calor.

Através do estudo da dinâmica de modos geodésicos de baixas frequências na seção 3.4, observamos que há três frequências típicas correspondentes a ZF $(\omega \sim 0)$, SW $\left(\omega \sim v_{T_{i}} / q R_{0}\right)$ e $\operatorname{GAM}\left(\omega \sim 2 v_{T_{i}} / R_{0}\right)$. O tipo de modo associado a cada uma destas frequências é importante porque descreve o processo físico envolvido. O primeiro, $\mathrm{ZF}$, ocorre quando o plasma responde de maneira incompressível à perturbação eletrostática, em contraste com os outros dois tipos, caracterizados por compressibilidade do plasma. Ondas de som (SW) só podem ocorrer em duas situações, na ausência de perturbações eletrostáticas ( $\tilde{\Phi}_{0}=$ const.) e quando há rotação poloidal de equilíbrio. Ainda não há na literatura uma compreensão detalhada sobre as razões físicas para o comportamento do plasma em relação às perturbações eletrostáticas ou eletromagnéticas. Como não ocorre uma transição suave entre os valores das frequências destes três tipos de modos, ocorrem lacunas ("gaps") no espectro de frequência e, portanto, a relação entre modos geodésicos e modos Alfvenicos [35] é uma importante área de investigação, principalmente no que se refere a diagnósticos, em especial, para a obtenção do perfil radial de $q(r)$ e da massa efetiva no plasma.

Ao incluir a componente do fluxo de calor decorrente do gradiente radial de temperatura na equação de conservação de energia, obtivemos a correção na frequência dos ZF, a qual, na presença de rotação poloidal assume valor não nulo e proporcional a $M_{P} / q$ [76]. Este importante resultado que obtivemos (publicado em 2011) e a expressão analítica detalhada para a frequência dos ZF é uma das contribuições desta tese.

O efeito da anisotropia de pressão de íons foi considerado no contexto da teoria de dois fluidos neste capítulo. Com a suposição de que a pressão ao longo das linhas de campo e perpendicular a estas, para íons, são diferentes, obtivemos o índice adiabático efetivo $\left(\gamma^{\text {(efetivo })}=7 / 4\right)$ para a frequência dos GAM. Entretanto, a correção devida a anisotropia de pressão iônica não é muito significativa, da ordem de 5\% no máximo. O efeito de rápida termalização de elétrons, ou seja, devido a sua pequena massa, estes se comportam isotermicamente e adiabaticamente, fazendo com que a temperatura de elétrons se torne constante em uma dada superfície magnética e, por esta razão, o efeito da anisotropia de elétrons pode ser desprezado. Para íons, no entanto, pelo 
fato de estes possuírem inércia muito maior, eles são incapazes entrarem em equilíbrio térmico no tempo característico de modos geodésicos.

Com relação a efeitos diamagnéticos, ou seja, efeitos causados por gradientes radiais de densidade e de temperatura de íons, os quais expressamos por meio dos comprimentos característicos $L_{N}$ e $L_{T_{i}}$, respectivamente, obtivemos dois GAM eletrostáticos. O primeiro apresenta um aumento de frequência devido a presença de gradiente de densidade. Em contraste, quando há fortes gradientes de temperatura, especificamente, $\eta_{i}=L_{N} / L_{T_{i}}>3 / 4$, o segundo modo se torna instável e não oscilatório. A taxa de crescimento desta instabilidade é proporcional à frequência de deriva de elétrons $\left(\omega_{* e}=T_{e} / \operatorname{er} B L_{N}\right)$ e, portanto, trata-se de um efeito do raio de Larmor finito (FLR), porém com relação aos íons. Este resultado, publicado recentemente [77], também é uma das principais contribuições desta tese. No capítulo seguinte, mostramos que em regiões de fator de segurança menor esta instabilidade pode ser suprimida.

Na seção 3.2 mostramos que gradientes de temperatura e rotação poloidal estão relacionados e, desta forma, considerar efeitos diamagnéticos (ou efeitos de deriva), ao invés de rotação de equilíbrio, pode ser uma alternativa conveniente para investigar a estabilidade de GAM. Esta conveniência reside na maior facilidade de utilização do modelo cinético, o qual descreve, entre outros efeitos exclusivos da teoria cinética, o amortecimento de Landau, o qual é discutido no próximo capítulo.

Considerar efeitos eletromagnéticos e efeitos diamagnéticos simultaneamente pode esclarecer questões importantes, entre elas, a discrepância no valor de baixas frequências obtidas experimentalmente com os valores teóricos das frequências de modos Alfvenicos e GAM. Além disso, o efeito do gradiente da temperatura de elétrons em modos geodésicos está ligado a perturbações magnéticas perpendiculares, descritas pela componente paralela do potencial vetor $\left(\tilde{A}_{\|}\right)$. Para a investigação de GAM eletromagnéticos [82] é necessário considerar segundos harmônicos $(m= \pm 2)$ nos modos poloidais, os quais desempenham um papel importante por contribuirem para a corrente paralela $\left(\tilde{j}_{\|}\right)[68]$.

Incluir simultaneamente rotação de equilíbrio, efeitos diamagnéticos e eletromagnéticos no estudo de GAM é uma proposta fora do escopo desta tese por levar a cálculos muito extensos, porém pretendemos levar adiante este estudo em um trabalho futuro a partir da metodologia 
descrita nesta tese. Também pretendemos considerar fluxo de calor na teoria de dois fluidos para analisar modos de frequências menores, em especial, fluxos zonais (ZF). 


\section{Capítulo 4}

\section{Investigação de modos acústicos}

\section{geodésicos (GAM) pelo modelo \\ girocinético}

Tendo em vista o que foi exposto nos capítulos 2 e 3, nos quais a discussão sobre o modelo giro-cinético e a aplicação do modelo de fluidos ao estudo de efeitos diamagnéticos nos modos acústicos geodésicos (GAM) são apresentados, investigamos, neste capítulo, efeitos diamagnéticos e amortecimento de Landau nos GAM utilizando a teoria giro-cinética.

\subsection{Estudo de GAM a partir do modelo girocinético}

Nesta seção, para facilitar a compreensão do modelo girocinétcio aplicado à dinâmica de GAM, por simplicidade, não levamos em conta gradientes de densidade e de temperatura.

Inicialmente, consideramos a função distribuição $\left(\tilde{f}_{\alpha}\right)$, que, conforme (2.54), é representada pela expressão

$$
\tilde{f}_{\alpha}=e_{\alpha} \tilde{\Phi} \frac{\partial F_{M_{\alpha}}}{\partial \mathcal{E}_{\alpha}}+\tilde{g}_{\alpha} \mathrm{e}^{i \mathbf{k}_{\perp} \cdot \boldsymbol{\rho}_{\alpha}}, \quad \boldsymbol{\rho}_{\alpha}=\frac{\mathbf{v}_{\perp} \times \mathbf{b}}{\omega_{c_{\alpha}}}
$$

onde $\tilde{g}_{\alpha}$ tem sua dinâmica governada pela equação girocinética, (2.52), a qual, quando despre- 
zados perturbações do campo magnético $(\tilde{\mathbf{B}} \approx 0)$ e gradientes de densidade e de temperatura $\left(\nabla F_{\mathrm{M} \alpha} \approx 0\right)$, pode ser escrita como

$$
\frac{\partial \tilde{g}_{\alpha}}{\partial t}+\left(\mathbf{v}_{g_{\alpha}} \cdot \nabla\right) \tilde{g}_{\alpha}=-e_{\alpha} \frac{\partial F_{M_{\alpha}}}{\partial \mathcal{E}_{\alpha}} \frac{\partial \tilde{\Phi}}{\partial t} J_{0}\left(k_{\perp} \rho_{\alpha}\right)
$$

A velocidade do centro guia, conforme (2.35), (2.37) e (2.38), quando não há rotação de equilíbrio $\left(\Phi_{0}=0\right)$, é obtida pela expressão:

$$
\mathbf{v}_{g_{\alpha}}=v_{\|} \mathbf{b}+\frac{\mathbf{b}}{\omega_{c_{\alpha}}} \times\left(\frac{v_{\perp}^{2}}{2} \nabla \ln B+v_{\|}^{2} \boldsymbol{\kappa}\right)
$$

que, em regimes de baixa pressão, ou seja, quando $\beta=\mathcal{O}\left(\varepsilon^{2}\right)$, e, para tokamaks de alta razão de aspecto $(\varepsilon \ll 1)$, pode ser aproximada por

$$
\mathbf{v}_{g_{\alpha}} \approx v_{\|} \mathbf{b}-\frac{1}{\omega_{c_{\alpha}} R_{0}}\left(\frac{v_{\perp}^{2}}{2}+v_{\|}^{2}\right)\left(\sin \theta \hat{\mathbf{e}}_{r}+\cos \theta \hat{\mathbf{e}}_{\theta}\right)
$$

Para desenvolver algebricamente (4.2) adotamos as seguintes substituições:

$$
\frac{\partial}{\partial t} \rightarrow-i \omega, \quad \nabla=i \hat{\mathbf{k}}, \quad \frac{\partial F_{M_{\alpha}}}{\partial \mathcal{E}_{\alpha}}=-\frac{F_{M_{\alpha}}}{T_{\alpha}}
$$

onde $F_{M_{\alpha}}$ é a função Maxwelliana que, em termos da energia da partícula $\left(\mathcal{E}_{\alpha}\right)$, é representada por (F.7) e $\hat{\mathbf{k}}=\hat{\mathbf{e}}_{r} \hat{k}_{r}+\mathbf{b} \hat{k}_{\|}$. Os operadores diferenciais (identificados por "^"), que constituem as componentes vetoriais de $\hat{\mathbf{k}}$, são definidos por:

$$
\begin{aligned}
& \hat{k}_{r}=-i \frac{\partial}{\partial r}, \quad \hat{k}_{\|}=\mathbf{b} \cdot\left(\hat{\mathbf{e}}_{\theta} \hat{k}_{\theta}+\hat{\mathbf{e}}_{\phi} \hat{k}_{\phi}\right)=-i k_{\|}\left(\frac{\partial}{\partial \theta}+q \frac{\partial}{\partial \phi}\right), \\
& \hat{k}_{\theta}=-i k_{\theta} \frac{\partial}{\partial \theta}, \quad \hat{k}_{\phi} \approx \frac{1}{R_{0}} \frac{\partial}{\partial \phi}, \quad k_{\theta}=\frac{1}{r}, \quad k_{\|}=\frac{1}{q R_{0}} .
\end{aligned}
$$

Da mesma forma como no capítulo 3 , consideramos a condição $\mathrm{k}_{r} \gg k_{\theta}$, condizente com a ordem MHD, o que justifica a aproximação $k_{\perp} \approx \mathrm{k}_{r}$ no argumento da função de Bessel, $J_{0}\left(k_{\perp} \rho_{\alpha}\right)$, em (4.2). Segue, então, que a eq. (4.2) pode ser aproximada para

$$
\left[\omega-\hat{k}_{\|} v_{\|}+\left(\frac{v_{\perp}^{2}}{2}+v_{\|}^{2}\right) \frac{\sin \theta}{\omega_{c_{\alpha}} R_{0}} \mathrm{k}_{r}\right] \tilde{g}_{\alpha}=\omega \frac{e_{\alpha}}{T_{\alpha}} J_{0}\left(\mathrm{k}_{r} v_{\perp} / \omega_{c_{\alpha}}\right) \tilde{\Phi} F_{M_{\alpha}}
$$


Com relação as variáveis $\theta$ e $\phi$, a função $\tilde{g}_{\alpha}$ pode ser expandida em série de Fourier,

$$
\tilde{g}_{\alpha}=\sum_{m, n} \tilde{g}_{m n}^{(\alpha)}(r) \mathrm{e}^{i(m \theta-n \phi)},
$$

onde $m$ e $n$ são, respectivamente, os modos poloidal e toroidal. Para GAM, $n=0$, de forma que a eq. (4.7) pode ser escrita como

$$
\sum_{m=-\infty}^{\infty}\left[i \frac{\Omega_{\mathrm{d} \alpha}}{2} \tilde{g}_{m-1}^{(\alpha)}+\left(1-m \Omega_{\mathrm{tr} \alpha}\right) \tilde{g}_{m}^{(\alpha)}-i \frac{\Omega_{\mathrm{d} \alpha}}{2} \tilde{g}_{m+1}^{(\alpha)}-F_{M_{\alpha}} J_{0 \alpha} \frac{e_{\alpha}}{T} \tilde{\Phi}_{m}\right] \mathrm{e}^{i m \theta}=0
$$

onde $J_{0 \alpha}=J_{0}\left(\mathrm{k}_{r} \rho_{\alpha}\right), \tilde{g}_{m}^{(\alpha)}=\tilde{g}_{m 0}^{(\alpha)}(r)$,

$$
\begin{gathered}
\Omega_{\mathrm{d} \alpha}=\left(\frac{1}{2} \frac{v_{\perp}^{2}}{v_{T_{\alpha}}^{2}}+\frac{v_{\|}^{2}}{v_{T_{\alpha}}^{2}}\right) \frac{\omega_{\mathrm{d} \alpha}}{\omega}, \quad \omega_{\mathrm{di}}=\mathrm{k}_{r} \rho_{i} \omega_{i}, \quad \omega_{\mathrm{de}}=-\tau_{e} \omega_{\mathrm{di}}, \quad \omega_{i}=\frac{v_{T_{i}}}{R_{0}}, \\
\Omega_{\operatorname{tr} \alpha}=\frac{v_{\|}}{v_{T_{\alpha}}} \frac{\omega_{\operatorname{tr} \alpha}}{\omega}, \quad \omega_{\operatorname{tr} i}=k_{\|} v_{T_{i}}=\omega_{i} / q, \quad \omega_{\operatorname{tr} e}=\sqrt{\tau_{e}\left(m_{i} / m_{e}\right)} \omega_{\operatorname{tr} i} \gg \omega_{\operatorname{tr} i} .
\end{gathered}
$$

Em (4.11), $\omega_{\text {tr } \alpha}$ é a frequência de circulação, que está associada ao movimento ao longo das linhas de campo magnético. O comprimento de onda relativo ao movimento paralelo pode ser representado pela expressão: $\lambda_{\|}=2 \pi q R_{0} /|m-n q|$, onde pode ser notado que $\lambda_{\|} \rightarrow \infty$ nas superfícies magnéticas racionais em que $q=m / n$. Superfícies magnéticas racionais são aquelas em que as linhas de campo se fecham sobre sí mesmas ( $q$ racional) e, por serem caracterizadas por instabilidades devidas a ressonâncias que nelas ocorrem, tem relevada importância em diversos fenômenos em física de tokamaks.

Para resolver (4.9), em uma primeira aproximação, podemos considerar $\tilde{g}_{m}=0$ para $|m| \geq 2$, ou seja, levando em conta apenas o efeito de primeiros harmônicos. Com o uso desta aproximação, que é válida para $\mathrm{k}_{r} \rho_{i} \ll 1$, obtemos a solução:

$$
\tilde{g}_{0}^{(\alpha)}=\frac{\left(J_{0 \alpha} e_{\alpha} / T_{\alpha}\right) F_{M_{\alpha}}}{1-\Omega_{\operatorname{tr} \alpha}^{2}-\Omega_{\mathrm{d} \alpha}^{2} / 2}\left[\left(1-\Omega_{\operatorname{tr} \alpha}^{2}\right) \tilde{\Phi}_{0}-i \frac{\Omega_{\mathrm{d} \alpha}}{2}\left(\tilde{\Phi}_{1}-\tilde{\Phi}_{-1}\right)-i \frac{\Omega_{\mathrm{d} \alpha} \Omega_{\mathrm{tr} \alpha}}{2}\left(\tilde{\Phi}_{1}+\tilde{\Phi}_{-1}\right)\right]
$$




$$
\tilde{g}_{ \pm 1}^{(\alpha)}=\frac{1}{1 \mp \Omega_{\operatorname{tr} \alpha}}\left( \pm i \frac{\Omega_{\mathrm{d} \alpha}}{2} \tilde{g}_{0}^{(\alpha)}+J_{0 \alpha} \frac{e_{\alpha}}{T} \tilde{\Phi}_{ \pm 1} F_{M_{\alpha}}\right)
$$

na qual o denominador de (4.12) pode ser aproximado por

$$
\frac{1}{1-\Omega_{\operatorname{tr} \alpha}^{2}-\Omega_{\mathrm{d} \alpha}^{2} / 2}=\frac{1}{1-\Omega_{\operatorname{tr} \alpha}^{2}}+\frac{1}{2} \frac{\Omega_{\mathrm{d} \alpha}^{2}}{\left(1-\Omega_{\mathrm{tr} \alpha}^{2}\right)^{2}}+\mathcal{O}\left(\Omega_{\mathrm{d} \alpha}^{4}\right)
$$

Na forma trigonométrica, as eqs. (4.12) e (4.13), a partir do uso das relações $\tilde{g}_{s}^{(\alpha)}=-i\left(\tilde{g}_{1}^{(\alpha)}-\tilde{g}_{-1}^{(\alpha)}\right)$ e $\tilde{g}_{c}^{(\alpha)}=\tilde{g}_{1}^{(\alpha)}+\tilde{g}_{-1}^{(\alpha)}$, podem ser expressas como:

$$
\begin{gathered}
\tilde{g}_{0}^{(\alpha)}=\frac{J_{0 \alpha} e_{\alpha} / T_{\alpha}}{1-\Omega_{\operatorname{tr} \alpha}^{2}}\left[\left(1+\frac{\Omega_{\mathrm{d} \alpha}^{2}}{2}-\Omega_{\operatorname{tr} \alpha}^{2}\right) \tilde{\Phi}_{0}+\frac{\Omega_{\mathrm{d} \alpha}}{2} \tilde{\Phi}_{s}+i \frac{\Omega_{\mathrm{d} \alpha} \Omega_{\mathrm{tr} \alpha}}{2} \tilde{\Phi}_{c}\right] F_{M_{\alpha}}, \\
\tilde{g}_{s}^{(\alpha)}=\frac{J_{0 \alpha} e_{\alpha} / T_{\alpha}}{1-\Omega_{\operatorname{tr} \alpha}^{2}}\left(\Omega_{\mathrm{d} \alpha} \tilde{\Phi}_{0}+\tilde{\Phi}_{s}+i \Omega_{\operatorname{tr} \alpha} \tilde{\Phi}_{c}\right) F_{M_{\alpha}}, \\
\tilde{g}_{c}^{(\alpha)}=\frac{J_{0 \alpha} e_{\alpha} / T_{\alpha}}{1-\Omega_{\operatorname{tr} \alpha}^{2}}\left[-i \Omega_{\mathrm{d} \alpha} \Omega_{\operatorname{tr} \alpha} \tilde{\Phi}_{0}-i \Omega_{\operatorname{tr} \alpha} \tilde{\Phi}_{s}+\tilde{\Phi}_{c}\right] F_{M_{\alpha}} .
\end{gathered}
$$

Ao longo desta tese, consideramos este modelo para a obtenção de modos de frequências no intervalo $\omega_{\text {tri }}<\omega \ll \omega_{\text {tre }}$. Não levamos em conta o efeito finito da massa de elétron, ou seja, $m_{e} / m_{i} \approx 0$, de forma que $\omega_{\text {tre }} \rightarrow \infty$. A seguir investigamos os efeitos do movimento paralelo das partículas, os quais estão relacionados com o valor da frequência $\omega_{\text {tri }}$. A metodologia que adotamos para o estudo deste movimento segue uma ordem gradativa de dificuldade com relação ao desenvolvimento algébrico. Desta forma, primeiramente consideramos $q \rightarrow \infty$ ou, seja, $\omega_{\text {tri }}=0$, entretanto a condição $m_{i} / q m_{e} \rightarrow \infty$ deve ser considerada simultaneamente, o que se traduz para $\omega_{\text {tre }} \rightarrow \infty$. Em um segundo passo, $\omega_{\text {tri }} / \omega \ll 1$ é considerado, assumindo que $\omega$ é real, o que nos permite obter correções $\mathcal{O}\left(q^{-2}\right)$ para a frequência dos GAM. Mesmo com esta consideração, ainda não estariamos levando em conta um importante efeito, a dissipação não colisional conhecida por amortecimento de Landau [33], o qual é incluido no final desta seção na terceira etapa. Nesta etapa, $\omega$ é visto como uma frequência complexa em princípio, e, em termos da função de dispersão de plasma [84], $Z(x)$, o resultado para $\omega_{\text {tri }} \sim \omega$ é obtido. 


\subsubsection{Limite de fluido com $k_{\|} v_{T_{i}}=0(q \rightarrow \infty)$}

Antes de iniciar o cálculo algébrico a partir da aproximação $k_{\|} v_{T_{i}}=0$, discutimos a respeito da densidade de partículas, a qual é utilizada na obtenção da relação de dispersão. A densidade de partículas, por sua vez, é obtida pela integração, no espaço de velocidades, da função distribuição, ou seja,

$$
\tilde{n}_{\alpha}=\left\langle\tilde{f}_{\alpha}\right\rangle=\tilde{n}_{\alpha}^{(C)}+\tilde{n}_{\alpha}^{(G)},
$$

onde, para uma grandeza genérica da forma $X_{\alpha}=X_{\alpha}\left(t, \mathbf{r}, v_{\perp}, v_{\|}, \gamma\right)$, definimos " \langle\rangle " como

$$
X_{\alpha}^{(\text {macroscópico })}(\mathbf{r}, t)=\langle X\rangle=\int_{v} d^{3} v X_{\alpha}^{(\text {partícula })}, \quad d^{3} v=d \gamma d v_{\|} d v_{\perp} v_{\perp} .
$$

Em (4.18), $\tilde{n}_{\alpha}^{(C)}$ e $\tilde{n}_{\alpha}^{(G)}$ representam, respectivamente, as contribuições cilíndrica (simétrica em relação a $\theta$ ) e geodésica (sensível a curvatura geodésica do campo magnético e não simétrica em relação a $\theta$ ) para a densidade de partículas. De acordo com (4.1), segue que

$$
\tilde{n}^{(C)}=e_{\alpha} \tilde{\Phi}\left\langle\frac{\partial F_{M_{\alpha}}}{\partial \mathcal{E}_{\alpha}}\right\rangle=-\frac{e_{\alpha} \tilde{\Phi}}{T_{\alpha}} F_{M_{\alpha}}, \quad \tilde{n}^{(G)}=\left\langle\tilde{g}_{\alpha} \mathrm{e}^{i \mathbf{k}_{\perp} \cdot \boldsymbol{\rho}_{\alpha}}\right\rangle
$$

Para elétrons, como $\omega_{\text {tre }} \rightarrow \infty, \Omega_{\text {tre }} \rightarrow \infty$ pode ser considerado em (4.15), (4.16) e (4.17), de forma que

$$
\tilde{g}_{0}^{(e)}=\frac{-e \tilde{\Phi}_{0}}{T_{e}} F_{M_{e}} \quad \text { e } \quad \tilde{g}_{s}^{(e)}=\tilde{g}_{c}^{(e)}=0
$$

e, consequentemente,

$$
\tilde{n}_{e}^{(C)}=\frac{e n_{0}}{T_{e}}\left(\tilde{\Phi}_{0}+\tilde{\Phi}_{s} \sin \theta+\tilde{\Phi}_{c} \cos \theta\right), \quad \tilde{n}_{e}^{(G)} \approx-\frac{e n_{0}}{T_{e}} \tilde{\Phi}_{0}
$$

Note que $\left\langle F_{M_{e}}\right\rangle=\left\langle F_{M_{i}}\right\rangle=n_{0}{ }^{1}$ e, com relação a contribuição geodésica, utilizamos a relação $\left\langle\exp \left(-i \mathbf{k}_{\perp} \cdot \boldsymbol{\rho}_{e}\right\rangle=J_{0}\left(k_{\perp} \rho_{e}\right) \approx 1\right.$, pois podemos desprezar o efeito de raio de Larmor finito (FLR)

\footnotetext{
${ }^{1}$ Este resultado é imediato se observarmos que a integral do primeiro momento da função distribuição é a densidade de partículas $\left(n_{0}\right)$ e se considerarmos a condição de quasi-neutralidade, $n_{i}=n_{e}=n_{0}$.
} 
para elétrons.

A contribuição cilíndrica para íons é analoga a de elétrons, ou seja, a densidade tem uma resposta de Boltzmann,

$$
\frac{\tilde{n}_{i}^{(C)}}{\tilde{n}_{e}^{(C)}}=-\tau_{e}, \quad \tau_{e}=\frac{T_{e}}{T_{i}} .
$$

No entanto, com relação à contribuição geodésica, a resposta é completamente diferente. Consideramos a seguir o caso $\omega_{\text {tri }} / \omega=0$ e, consequentemente $\Omega_{\text {tri }}=0$, de acordo com (4.11), de forma que o resultado para $\tilde{n}_{i}(G)$ pode ser expresso como:

$$
\tilde{n}_{i}^{(G)}=\frac{e n_{0}}{T_{i}}\left\{\left[\left(I_{0}^{(i)}+\frac{I_{2}^{(i)}}{2}\right) \tilde{\Phi}_{0}+\frac{I_{0}^{(i)}}{2} \tilde{\Phi}_{s}\right]+\left[I_{1}^{(i)} \tilde{\Phi}_{0}+I_{0}^{(i)} \tilde{\Phi}_{s}\right] \sin \theta+I_{0}^{(i)} \tilde{\Phi}_{c} \cos \theta\right\},
$$

onde as integrais $I_{n}^{(i)}=\left\langle J_{0 i}^{2}\left(\Omega_{\mathrm{di}} / \omega\right)^{n}\right\rangle, n=0,1,2$ são calculadas em F.2 e podem ser aproximadas por: $I_{0}^{(i)}=1-\mathrm{k}_{r}^{2} \rho_{i}^{2} / 2, I_{1}^{(i)}=\mathrm{k}_{r} \rho_{i} / \Omega$ e $I_{2}^{(i)}=(7 / 4) \mathrm{k}_{r}^{2} \rho_{i}^{2} / \Omega^{2}$ em (4.24). Note que, analogamente ao capítulo anterior, consideramos a frequência normalizada, mas definida de forma diferente aqui, ou seja,

$$
\Omega=\frac{\omega}{\omega_{i}}=\frac{\omega R_{0}}{v_{T_{i}}} .
$$

Segue, portanto, que o cálculo da densidade perturbada total de íons e de elétrons, em primeira ordem, resulta em

$$
\begin{aligned}
& \frac{\tilde{n}_{i}}{e n_{0} / T_{i}}=\frac{1}{2}\left[\left(-\Omega^{2}+7 / 4\right) \frac{\mathrm{k}_{r}^{2} \rho_{i}^{2}}{\Omega^{2}} \tilde{\Phi}_{0}+\frac{\mathrm{k}_{r} \rho_{i}}{\Omega} \tilde{\Phi}_{s}\right]+\frac{\mathrm{k}_{r} \rho_{i}}{\Omega} \tilde{\Phi}_{0} \sin \theta, \\
& \frac{\tilde{n}_{e}}{e n_{0} / T_{i}}=\tau_{i}\left(\tilde{\Phi}_{s} \sin \theta+\tilde{\Phi}_{c} \cos \theta\right), \quad \tau_{i}=\frac{T_{i}}{T_{e}} .
\end{aligned}
$$

Para obter a frequência dos GAM, empregamos a condição de quasi-neutralidade,

$$
e\left(\tilde{n}_{i}-\tilde{n}_{e}\right)=0
$$

na qual substituímos os valores das densidades perturbadas de íons e de elétrons mostrados em 
(4.26), o que resulta na equação:

$$
\frac{1}{2}\left[\left(-\Omega^{2}+\frac{7}{4}\right) \mathrm{k}_{r}^{2} \rho_{i}^{2} \tilde{\Phi}_{0}+\Omega \mathrm{k}_{r} \rho_{i} \tilde{\Phi}_{s}\right]+\left(\Omega \mathrm{k}_{r} \rho_{i} \tilde{\Phi}_{0}-\tau_{i} \Omega^{2} \tilde{\Phi}_{s}\right) \sin \theta-\tau_{i} \Omega^{2} \tilde{\Phi}_{c} \cos \theta=0
$$

que é válida para $\Omega \neq 0$ e é satisfeita para $\theta$ arbitrário se, e somente se,

$$
\tilde{\Phi}_{c}=0, \quad \tilde{\Phi}_{s}=\tau_{e} \frac{\mathrm{k}_{r} \rho_{i}}{\Omega} \tilde{\Phi}_{0}, \quad\left(-\Omega^{2}+\frac{7}{4}+\tau_{e}\right) \mathrm{k}_{r}^{2} \rho_{i}^{2} \tilde{\Phi}_{0}=0
$$

Note que (4.28) e (4.29) sugerem a relação $\tilde{\Phi}_{m}=\mathcal{O}\left(\mathrm{k}_{r}^{m} \rho_{i}^{m}\right) \tilde{\Phi}_{0}$, a qual pode ser utilizada em modelos mais abrangentes. A última relação em (4.29) resulta na frequência dos GAM,

$$
\omega_{\mathrm{GAM}}=\Omega_{\mathrm{g} 0} \frac{v_{T_{i}}}{R_{0}}, \quad \Omega_{\mathrm{g} 0}^{2}=\frac{7}{4}+\frac{T_{e}}{T_{i}} .
$$

a qual concorda com o valor obtido pela teoria de dois fluidos com viscosidade paralela, conforme (3.122).

A seguir, considerando $k_{\|} v_{T_{i}}$ finito, porém $k_{\|} v_{T_{i}} \ll \omega$, obtemos correções de $\mathcal{O}\left(q^{-2}\right)$ para a frequência mostrada em (4.30).

\subsubsection{Limite de fluido com $k_{\|} v_{T_{i}}$ finito $(q \gg 1)$}

No limite de fluido, utilizamos a seguinte expansão em série,

$$
\frac{1}{1-\Omega_{\mathrm{tri}}^{2}}=1+\Omega_{\mathrm{tri}}^{2}+\mathcal{O}\left(\Omega_{\mathrm{tri}}^{4}\right)
$$

em (4.15), (4.16) e (4.17) e, ao desprezar termos de $\mathcal{O}\left(\Omega_{\text {tri }}^{3}\right)$, a relação de dispersão, escrita na forma matricial, fica

$$
\frac{e^{2} n_{0}}{T_{i}}\left(\begin{array}{ccc}
1 & \sin \theta & \cos \theta
\end{array}\right)\left(\begin{array}{ccc}
\mathcal{R}_{00} & \mathcal{R}_{0 s} & \mathcal{R}_{0 c} \\
\mathcal{R}_{s 0} & \mathcal{R}_{s s} & \mathcal{R}_{s c} \\
\mathcal{R}_{c 0} & \mathcal{R}_{c s} & \mathcal{R}_{c c}
\end{array}\right)\left(\begin{array}{c}
\tilde{\Phi}_{0} \\
\tilde{\Phi}_{s} \\
\tilde{\Phi}_{c}
\end{array}\right)=0
$$


onde os elementos da matriz central são definidos pelas seguintes expressões:

$$
\begin{aligned}
& \mathcal{R}_{00}=I_{00}^{(i)}-1+\frac{1}{2}\left(I_{20}^{(i)}+I_{22}^{(i)}\right)=\frac{1}{2}\left[-\Omega^{2}+\frac{7}{4}+\frac{23}{8} \frac{1}{q^{2} \Omega^{2}}\right] \frac{\mathrm{k}_{r}^{2} \rho_{i}^{2}}{\Omega^{2}}, \\
& \mathcal{R}_{0 s}=\frac{1}{2} \mathcal{R}_{s 0}=\frac{1}{2}\left(I_{10}^{(i)}+I_{12}^{(i)}\right)=\frac{1}{2}\left(1+\frac{1}{q^{2} \Omega^{2}}\right) \frac{\mathrm{k}_{r} \rho_{i}}{\Omega}, \\
& \mathcal{R}_{s s}=\mathcal{R}_{c c}=I_{02}^{(i)}-\tau_{i}=\frac{1}{2 q^{2} \Omega^{2}}-\tau_{i}, \\
& \mathcal{R}_{0 c}=-\frac{1}{2} \mathcal{R}_{c 0}=\frac{i}{2} I_{11}^{(i)}=0, \quad \mathcal{R}_{s c}=-\mathcal{R}_{c s}=i I_{01}^{(i)}=0,
\end{aligned}
$$

onde as integrais da forma $I_{a b}^{(i)}=\left\langle J_{0 i}^{2} \Omega_{\mathrm{di}}^{a} \Omega_{\mathrm{tri}}^{b}\right\rangle$, com $a, b=0,1,2$, são computadas em F.2. Para que não haja a solução trivial, impõe-se que o determinante da matriz central de (4.32) seja nulo, ou seja,

$$
\mathcal{R}_{s s}^{2}\left(\mathcal{R}_{00}-2 \frac{\mathcal{R}_{0 s}^{2}}{\mathcal{R}_{s s}}\right)=0
$$

Da condição $\mathcal{R}_{s s}=0$ obtemos a frequência sigular no ramo acústico de íons:

$$
\omega_{s w 0}=\Omega_{\mathrm{s} 0} \frac{v_{T_{i}}}{R_{0}}=k_{\|} c_{\mathrm{s} e}, \quad \Omega_{\mathrm{s} 0}^{2}=\frac{\tau_{e}}{2 q^{2}},
$$

onde $c_{\mathrm{se}}=\sqrt{T_{e} / m_{i}}$ é a velocidade do som no limite de íons frios $\left(T_{i} \ll T_{e}\right)$, pois $c_{s}^{2}=$ $\sqrt{\left(\gamma_{i} T_{i}+\gamma_{e} T_{e}\right) / m_{i}}$ e $\gamma_{e}=1$.

Para $\mathcal{R}_{s s} \neq 0$, segue que

$$
\left(-\Omega^{2}+\frac{7}{4}+\tau_{i} \frac{23}{4} \frac{\Omega_{\mathrm{s} 0}^{2}}{\Omega^{2}}+\frac{\tau_{e}+4 \Omega_{\mathrm{s} 0}^{2} / \Omega^{2}}{1-\Omega_{\mathrm{s} 0}^{2} / \Omega^{2}}\right) \frac{\mathrm{k}_{r}^{2} \rho_{i}^{2}}{\Omega^{2}}=0 .
$$

Note que no limite $\Omega_{\mathrm{s} 0} \rightarrow 0(q \rightarrow \infty)$, a solução mostrada em (4.30) é obtida. Considerando $\mathrm{k}_{r} \rho_{i} \neq 0$, as soluções de (4.36) em $\Omega^{2}$ são obtidas a partir da seguinte equação bi-cúbica:

$$
\Omega^{6}-\left(\Omega_{\mathrm{g} 0}^{2}+\Omega_{\mathrm{s} 0}^{2}\right) \Omega^{4}-\left(\frac{23}{4} \tau_{i}+\frac{9}{4}\right) \Omega_{\mathrm{s} 0}^{2} \Omega^{2}+\frac{23}{4} \tau_{i} \Omega_{\mathrm{s} 0}^{4}=0, \quad \tau_{i}=\frac{T_{i}}{T_{e}} .
$$

Pelo fato de não levarmos em conta gradientes de densidade e de temperatura (fontes de instabilidades) e efeitos cinéticos nesta seção, as únicas soluções de (4.37) com significado físico são as positivas. Estas soluções podem ser determinadas analiticamente de forma aproximada, 
pois elas possuem ordens de grandezas distintas: $\Omega_{1} \sim 1$ e $\Omega_{2} \sim \Omega_{\mathrm{s} 0}$, onde $\Omega_{\mathrm{s} 0} \ll 1$. Para a determinação da primeira, podemos desprezar o último termo do lado esquerdo em (4.37), que é de $\mathcal{O}\left(\Omega_{\mathrm{s} 0}^{4}\right)$. Com relação à segunda solução, podemos desprezar $\Omega^{6}$ em (4.37), obtendo também uma equação bi-quadrática. No primeiro caso, a equação a ser resolvida é dada por:

$$
\Omega^{4}-\left(\Omega_{\mathrm{g} 0}^{2}+\Omega_{\mathrm{s} 0}^{2}\right) \Omega^{2}-\left(\frac{23}{4} \tau_{i}+\frac{9}{4}\right) \Omega_{\mathrm{s} 0}^{2}=0 .
$$

Das soluções de (4.38),

$$
\Omega^{2}=\frac{\Omega_{\mathrm{g} 0}^{2}}{2}\left[1+\left(\frac{7}{4}+\tau_{e}\right) \frac{\Omega_{\mathrm{s} 0}^{2}}{\Omega_{\mathrm{g} 0}^{2}}\right]\left[1 \pm \sqrt{1+\frac{\left(23 \tau_{i}+9\right) \Omega_{\mathrm{s} 0}^{2} / \Omega_{\mathrm{g} 0}^{2}}{\left(1+\Omega_{\mathrm{s} 0}^{2} / \Omega_{\mathrm{g} 0}^{2}\right)^{2}}}\right]
$$

a única com significado físico, que representa GAM com correções em $q$, é a solução positiva, a qual pode ser aproximada quando $\Omega_{\mathrm{s} 0} / \Omega_{\mathrm{g} 0} \ll 1$, ou seja,

$$
\omega_{G A M}=\left(\Omega_{\mathrm{g} 0}^{2}+\frac{\Omega_{\mathrm{s} 1}^{2}}{\Omega_{\mathrm{g} 0}^{2}}\right)^{1 / 2} \frac{v_{T_{i}}}{R_{0}}+\mathcal{O}\left(q^{-4}\right) \frac{v_{T_{i}}}{R_{0}},
$$

onde

$$
\Omega_{\mathrm{s} 1}=\left(\frac{23}{4} \tau_{i}+4+\tau_{e}\right)^{1 / 2} \Omega_{\mathrm{s} 0}
$$

Admitindo $\Omega \sim \Omega_{\mathrm{s} 0}$, a eq. (4.37) pode ser aproximada para

$$
\Omega^{4}+\left(\frac{23}{4} \tau_{i}+\frac{9}{4}\right) \frac{\Omega_{\mathrm{s} 0}^{2}}{\Omega_{\mathrm{g} 0}^{2}} \Omega^{2}-\frac{23}{4} \tau_{i} \frac{\Omega_{\mathrm{s} 0}^{2}}{\Omega_{\mathrm{g} 0}^{2}} \Omega_{\mathrm{s} 0}^{2}=0
$$

cuja solução válida, que representa SWs, é dada por

$$
\omega_{S W}=\left[\sqrt{1+\frac{4\left(7+4 \tau_{e}\right) \tau_{e}}{23\left(1+9 \tau_{e} / 23\right)^{2}}}-1\right]^{1 / 2}\left(\frac{23}{8} \tau_{i}+\frac{9}{8}\right)^{1 / 2} \frac{\Omega_{\mathrm{s} 0}}{\Omega_{\mathrm{g} 0}} \frac{v_{T_{i}}}{R_{0}}
$$

Note que no limite de íons frios, $\tau_{e} \rightarrow \infty, \Omega_{\mathrm{SW}} \rightarrow 1 / q$, em acordo com a teoria da MHD ideal se considerado $\gamma_{e}=1$. No entanto, para $\tau_{i}$ finito, $\Omega_{\mathrm{SW}}<1 / q$, de forma que, no espectro de frequência, esta solução está abaixo da frequência acústica de ions. 
Com a substituição dos valores de $\Omega_{\mathrm{g} 0}, \Omega_{\mathrm{s} 0}$ e $\Omega_{\mathrm{s} 1}$ em (4.40), obtemos a forma final da frequência de GAM,

$$
\omega_{\mathrm{GAM}}=\left[\left(\frac{7}{4}+\frac{T_{e}}{T_{i}}\right)+\frac{1}{q^{2}}\left(\frac{23}{8}+2 \frac{T_{e}}{T_{i}}+\frac{1}{2} \frac{T_{e}^{2}}{T_{i}^{2}}\right)\left(\frac{7}{4}+\frac{T_{e}}{T_{i}}\right)^{-1}\right]^{1 / 2} \frac{v_{T_{i}}}{R_{0}}
$$

que está de acordo com o resultado obtido anteriormente em [66,82] (veja, nestas Refs., as eqs. (6) e (46) respectivamente).

No limite de íons frios $\left(T_{i} \ll T_{e}\right)$, a solução apresentada em (4.44) converge para o valor obtido pelo modelo da MHD ideal [26], se considerarmos $\gamma_{e}=1$, de acordo com (3.99) e (3.100). Esta observação foi feita anteriormente por A. I. Smolyakov [82], e, neste caso, a frequência pode ser aproximada por:

$$
\omega_{\mathrm{GAM}} \approx\left(2+\frac{1}{q^{2}}\right)^{1 / 2} \frac{c_{\mathrm{s} e}}{R_{0}}, \quad c_{\mathrm{s} e}=\sqrt{\frac{T_{e}}{m_{i}}} .
$$

A partir de (4.32), (4.33) e (4.44) segue que

$$
\tilde{\Phi}_{c}=0, \quad \tilde{\Phi}_{s}=-2 \frac{\mathcal{R}_{0 s}}{\mathcal{R}_{s s}} \tilde{\Phi}_{0}=\frac{\tau_{e} \Omega^{2}+2 \Omega_{\mathrm{s} 0}^{2}}{\Omega^{2}-\mathrm{k}_{r} \rho_{\mathrm{s} 0}} \frac{\rho_{i}}{\Omega} \tilde{\Phi}_{0},
$$

onde pode ser notado que $\Omega=\Omega_{\mathrm{s} 0}$, assim como em (4.36), representa uma singularidade.

\subsubsection{Dissipação de Landau em GAM $\left(\omega>k_{\|} v_{T_{i}}\right)$}

Para valores baixos de $q$, a expansão em série, em (4.31), não pode ser utilizada, de forma que ao desenvolver as equações os resultados dependerão da função de dispersão de plasma [84], que é definida como:

$$
Z(\zeta)=\frac{1}{\sqrt{\pi}} \int_{-\infty}^{\infty} d x \frac{\mathrm{e}^{-x^{2}}}{x-\zeta}
$$

onde $x$ é real e $\zeta$ complexo, com sua parte imaginária, $\mathcal{I} m(\zeta)$, podendo assumir valores positivo, negativo ou nulo, mediante o método de prolongamento analítico. Para a obtenção de resultados analíticos, considera-se $|\mathcal{I} m(\zeta)| \ll|\mathcal{R} e(\zeta)|$, de forma que este modelo se aplica exclusivamente 
ao estudo de modos fracamente instáveis ou amortecidos.

O cálculo dos termos da matriz central de (4.32), em termos da função dispersão, resulta em:

$$
\begin{aligned}
& \mathcal{R}_{00}=\mathcal{L}_{0}^{(i)}-1+\frac{1}{2} \mathcal{L}_{2}^{(i)}-\mathcal{L}_{02}^{(i)}=-\frac{1}{2}\left\{\frac{3}{2} \zeta_{\alpha}^{2}+\zeta_{\alpha}^{4}+\left(\frac{1}{2} \zeta_{\alpha}+\zeta_{\alpha}^{3}+\zeta_{\alpha}^{5}\right)\left[Z\left(\zeta_{\alpha}\right)-i \sqrt{\pi} \mathrm{e}^{\left.-\zeta_{i}^{2}\right]} \frac{\mathrm{k}_{r}^{2} \rho_{i}^{2}}{\Omega^{2}},\right.\right. \\
& \mathcal{R}_{0 s}=\frac{1}{2} \mathcal{R}_{s 0}=\frac{1}{2} \mathcal{L}_{1}^{(i)}=-\frac{1}{2}\left\{\zeta_{i}^{2}+\left(\frac{1}{2} \zeta_{i}+\zeta_{i}^{3}\right)\left[Z\left(\zeta_{i}\right)-i \sqrt{\pi} \mathrm{e}^{-\zeta_{i}^{2}}\right]\right\} \frac{\mathrm{k}_{r} \rho_{i}}{\Omega}, \\
& \mathcal{R}_{s s}=\mathcal{R}_{c c}=\mathcal{L}_{0}^{(i)}-1-\tau_{i}=-\left\{1+\tau_{i}+\zeta_{i}\left[Z\left(\zeta_{i}\right)-i \sqrt{\pi} \mathrm{e}^{-\zeta_{i}^{2}}\right]\right\} \\
& \mathcal{R}_{0 c}=-\frac{1}{2} \mathcal{R}_{c 0}=\frac{i}{2} \mathcal{L}_{11}^{(i)} \propto \mathrm{e}^{-\zeta_{i}^{2}}, \quad \mathcal{R}_{s c}=-\mathcal{R}_{c s}=i \mathcal{L}_{01}^{(i)} \propto \mathrm{e}^{-\zeta_{i}^{2}},
\end{aligned}
$$

onde $\zeta_{i}=q \Omega$ e $\Omega=\Omega_{R}+i \Gamma$. Em (4.48) utilizamos as seguintes definições:

$$
\mathcal{L}_{a}^{(\alpha)}=\left\langle\frac{J_{0 \alpha}^{2} \Omega_{\mathrm{d} \alpha}^{a}}{1-\Omega_{\mathrm{tr} \alpha}^{2}}\right\rangle \quad \text { e } \quad \mathcal{L}_{a b}^{(\alpha)}=\left\langle\frac{J_{0 \alpha}^{2} \Omega_{\mathrm{d} \alpha}^{a} \Omega_{\mathrm{tr} \alpha}^{b}}{1-\Omega_{\mathrm{tr} \alpha}^{2}}\right\rangle, \quad a, b=0, s, c,
$$

cujos cálculos são efetuados em F.3 e F.4.

A relação de dispersão,

$$
\mathcal{R}_{s s}^{2}\left[\left(1+\frac{\mathcal{R}_{s c}^{2}}{\mathcal{R}_{s s}^{2}}\right) \mathcal{R}_{00}-2 \frac{\left(\mathcal{R}_{0 s}^{2}-\mathcal{R}_{0 c}^{2}\right)}{\mathcal{R}_{s s}}-4 \frac{\mathcal{R}_{0 s} \mathcal{R}_{0 c} \mathcal{R}_{s c}}{\mathcal{R}_{s s}^{2}}\right]=0
$$

é obtida a partir de (4.32) e (4.48), onde os valores de $\mathcal{R}_{a b}$ são calculados considerando-se a forma aproximada de $Z\left(\zeta_{i}\right)$, conforme (F.24), para $\zeta_{i} \gg 1$. Com este procedimento, a partir da substituição dos termos dominantes em (4.50),

$$
\begin{aligned}
& \mathcal{R}_{00}=\frac{1}{2}\left[-\Omega^{2}+\left(\frac{7}{4}+\frac{23}{8} \frac{1}{\zeta_{i}^{2}}+i(\sigma-1) \sqrt{\pi} \zeta_{i}^{5} \mathrm{e}^{-\zeta_{i}^{2}}+i(\sigma-1) \sqrt{\pi} \zeta_{i}^{3} \mathrm{e}^{-\zeta_{i}^{2}}\right)\right] \frac{\mathrm{k}_{r}^{2} \rho_{i}^{2}}{\Omega^{2}} \\
& \mathcal{R}_{0 s}=\frac{1}{2}\left(1+\frac{1}{\zeta_{i}^{2}}+i(\sigma-1) \sqrt{\pi} \zeta_{i}^{3} \mathrm{e}^{-\zeta_{i}^{2}}\right) \frac{\mathrm{k}_{r} \rho_{i}}{\Omega}, \quad \mathcal{R}_{s s}=-\left(\tau_{i}-\frac{1}{2 \zeta_{i}^{2}}+i(\sigma-1) \sqrt{\pi} \zeta_{i} \mathrm{e}^{-\zeta_{i}^{2}}\right)
\end{aligned}
$$

em (4.50), não levando em conta termos de ordem $\mathrm{e}^{-2 \zeta_{i}^{2}}$, resulta a forma final:

$$
-\Omega^{2}+\Omega_{\mathrm{g} 0}^{2}+\frac{\Omega_{\mathrm{s} 1}^{2}}{\Omega^{2}}+i(\sigma-1)\left[1+2\left(2+\tau_{i}\right) \frac{\Omega_{\mathrm{s} 0}^{2}}{\Omega^{2}}\right] \sqrt{\pi} q^{5} \Omega^{5} \mathrm{e}^{-q^{2} \Omega^{2}}=0 .
$$

Note que para $\sigma=1$ retomamos o limite de fluido, cujos resultados foram apresentados anteriormente. Com a escolha correta, $\sigma=2$, que representa o caso em que ocorre amortecimento de 
Landau, obtemos a relação correta.

A razão física para que estes modos sejam amortecidos é baseada no fato de que o integrando da integral com relação a velocidade paralela é uma função gaussiana e, portanto, o número de partículas com velocidades inferiores à velocidade de fase da onda $\left(v_{p h}\right)$, a qual está localizada na cauda da distribuição, é maior do que o número de partículas com velocidades superiores a $v_{p h}$. Em consequência, há mais partículas que recebem energia da onda do que partículas que cedem energia a ela, de forma que, com a perda de energia, a onda é amortecida devido a interação onda-partícula média, ou efeito de Landau [33].

Nesta seção, pelo fato de estarmos interessados nos valores de frequências e taxas de amortecimento somente em primeira ordem, os quais são apresentados incluindo a próxima ordem na seção 4.3, considerando $\Omega=\Omega_{R}+i \Gamma$, com $\Gamma<0$ e $|\Gamma| \ll\left|\Omega_{R}\right|$, a equação a ser resolvida pode ser aproximada por:

$$
\begin{gathered}
D(\Omega)=\mathcal{F}(\Omega)+i \mathcal{K}(\Omega)=0, \\
\mathcal{F} \approx \Omega^{2}-\left(7 / 4+\tau_{e}\right), \quad \mathcal{K} \approx \sqrt{\pi} q^{5} \Omega^{5} \mathrm{e}^{-q^{2} \Omega^{2}}
\end{gathered}
$$

Para resolver (4.53), utilizamos um procedimento iterativo baseado na expansão em série de Taylor para $\Omega=\Omega_{R}$ em primeira ordem. Inicialmente consideramos a aproximação em série:

$$
D(\Omega) \approx \mathcal{F}\left(\Omega_{R}\right)+i \mathcal{K}\left(\Omega_{R}\right)+\left.i \Gamma\left(\frac{\partial \mathcal{F}}{\partial \Omega}+i \frac{\partial \mathcal{K}}{\partial \Omega}\right)\right|_{\Omega=\Omega_{R}}=0
$$

Posteriormente, isolando parte real de imaginária em (4.54), onde ambas devem se anular, obtemos as relações:

$$
\begin{gathered}
F\left(\Omega_{R}\right)=0, \\
\Gamma=-\frac{\mathcal{K}\left(\Omega_{R}\right)}{\mathcal{F}^{\prime}\left(\Omega_{R}\right)}, \quad \mathcal{F}^{\prime}=\frac{\partial \mathcal{F}}{\partial \Omega},
\end{gathered}
$$

nas quais desprezamos o termo $-\Gamma \mathcal{K}^{\prime}\left(\Omega_{R}\right)$ em (4.55) por ser muito pequeno. $\mathrm{O}$ último passo, 
consiste em resolver (4.55) para obter $\Omega_{R}$ e, finalmente, substitui-lo em (4.56), o que permite a obtenção de $\Gamma$.

Em uma primeira aproximação, segue que

$$
\Omega_{R} \approx \sqrt{\frac{7}{4}+\tau_{e}}, \quad \Gamma \approx-\frac{\sqrt{\pi}}{2} q^{5} \Omega_{R}^{4} \mathrm{e}^{-q^{2} \Omega_{R}^{2}}
$$

É possível avaliar o modelo por meio do cálculo numérico de $\Gamma / \Omega_{R}$. Para $q=1$ e $\tau_{e}=3$, ou seja, próximo do centro da coluna de plasma, $|\Gamma| / \Omega_{R} \approx 0.17$. Conforme nos aproximamos da borda, este valor diminui drasticamente. Por exemplo, supondo $q=2, \tau_{e}=1,|\Gamma| / \Omega_{R} \sim 10^{-4}$. Concluímos, portanto, que o amortecimento de GAM é importante apenas para $q$ baixo, o que normalmente ocorre no centro da coluna de plasma e, a partir deste modelo, o efeito do amortecimento de Landau para íons é teoricamente descrito com bastante precisão. O resultado que obtivemos para $\Gamma$ concorda com o obtido em [66,85].

\subsection{Discussão sobre aplicações do modelo girocinético na forma mais geral}

A seguir apresentamos uma discussão mais detalhada sobre o modelo girocinético, no qual consideramos o gradiente radial da função Maxwelliana e o potencial vetor paralelo perturbado $\left(\tilde{A}_{\|}\right)$, o qual descreve perturbações magnéticas perpendiculares. Neste contexto, a equação girocinética a ser desenvolvida é expressa por:

$$
\frac{\partial \tilde{g}_{\alpha}}{\partial t}+\left(\mathbf{v}_{g_{\alpha}} \cdot \nabla\right) \tilde{g}_{\alpha}=e_{\alpha}\left(-\frac{\partial F_{M_{\alpha}}}{\partial \mathcal{E}_{\alpha}} \frac{\partial}{\partial t}+\frac{\mathbf{b} \times \nabla F_{M_{\alpha}}}{m_{\alpha} \omega_{c_{\alpha}}} \cdot i \mathbf{k}_{\perp}\right) J_{0 \alpha}\left(\tilde{\Phi}-v_{\|} \tilde{A}_{\|}\right),
$$

ao invés da versão apresentada em (4.2). Entretanto $\tilde{f}_{\alpha}$, em termos de $\tilde{g}_{\alpha}$, permanece inalterada e, de acordo com (4.1), é expressa como:

$$
\tilde{f}_{\alpha}=e_{\alpha} \tilde{\Phi} \frac{\partial F_{M_{\alpha}}}{\partial \mathcal{E}_{\alpha}}+\tilde{g}_{\alpha} \mathrm{e}^{i \mathbf{k}_{\perp} \cdot \boldsymbol{\rho}_{\alpha}}
$$


Com a utilização de (F.8) e (F.10) em (4.58) resulta que para qualquer $m$ e $n$, deve ser satisfeita a seguinte equação:

$$
-i \frac{\Omega_{\mathrm{d} \alpha}}{2} \tilde{g}_{m-1, n}^{(\alpha)}+\left[1-(m-n q) \Omega_{\mathrm{tr} \alpha}\right] \tilde{g}_{m, n}^{(\alpha)}+i \frac{\Omega_{\mathrm{d} \alpha}}{2} \tilde{g}_{m+1, n}^{(\alpha)}=\left(1-m \Omega_{*}\right) \tilde{\Psi}_{m, n}^{(\alpha)},
$$

onde

$$
\begin{gathered}
\tilde{\Psi}_{m, n}^{(\alpha)}=J_{0 \alpha}\left(\tilde{\Phi}_{m, n}-v_{\|} \tilde{A}_{\| m, n}\right) \frac{e_{\alpha}}{T_{\alpha}} F_{M_{\alpha}} \\
\Omega_{* \alpha}=\frac{e_{\alpha}}{e} \frac{\omega_{* \alpha}}{\omega}\left[1+\eta_{\alpha}\left(\frac{v_{\perp}^{2}}{v_{T_{\alpha}}^{2}}+\frac{v_{\|}^{2}}{v_{T_{\alpha}}^{2}}-\frac{3}{2}\right)\right], \quad \omega_{* \alpha}=\frac{T_{\alpha}}{e B r L_{N}} \sim \frac{\rho_{i} / L_{N}}{r / R_{0}} \frac{v_{T_{i}}}{R_{0}} .
\end{gathered}
$$

A relação entre $\tilde{A}_{\|}$e $\tilde{\Phi}$ é obtida pela lei de Ampère, ou seja, considerando $\mathrm{k}_{r} \gg r^{-1}$, resulta que

$$
\tilde{J}_{\|}=\sum_{\alpha=i, e} e_{\alpha}\left\langle v_{\|} \tilde{f}_{\alpha}\right\rangle \approx \frac{\mathrm{k}_{r}^{2}}{\mu_{0}} \tilde{A}_{\|},
$$

onde $\tilde{f}_{\alpha}=\tilde{f}_{\alpha}\left(\tilde{\Phi}, \tilde{A}_{\|}\right)$no caso mais geral.

A equação (4.60), em conjunto com a relação (4.63), é o ponto de partida para a investigação de inúmeros tipos de modos Alfvênicos e geodésicos e seus correspondentes automodos. Com estas equações podemos tratar diversos efeitos, tais como efeitos eletromagnéticos $\left(\tilde{A}_{\|} \neq 0\right)$, efeitos de deriva $\left(\omega_{* \alpha} \neq 0\right)$, amortecimento de Landau, etc. No entanto, seguindo o foco desta tese, consideramos apenas efeitos de deriva e amortecimento de Landau, os quais são apresentados na próxima seção. Em futuros trabalhos pretendemos desenvolver os outros tópicos mencionados. 


\subsection{Efeitos diamagnéticos e amortecimento de Landau em GAM}

Se considerarmos apenas primeiros harmônicos no regime eletrostático, incluindo gradientes de temperatura e de densidade provenientes do gradiente da função distribuição de equilíbrio, as soluções de (4.60) se resumem a

$$
\begin{gathered}
\tilde{g}_{0}^{(\alpha)}=\frac{J_{0 \alpha} e_{\alpha} F_{M_{\alpha}} / T_{\alpha}}{1-\Omega_{\operatorname{tr} \alpha}^{2}-\Omega_{\mathrm{d} \alpha}^{2} / 2}\left[i \frac{\Omega_{\mathrm{d} \alpha}}{2}\left(1-\Omega_{\operatorname{tr} \alpha}\right)\left(1+\Omega_{* \alpha}\right) \tilde{\Phi}_{-1}+\right. \\
\left.\left(1-\Omega_{\operatorname{tr} \alpha}^{2}\right) \tilde{\Phi}_{0}-i \frac{\Omega_{\mathrm{d} \alpha}}{2}\left(1+\Omega_{\operatorname{tr} \alpha}\right)\left(1-\Omega_{* \alpha}\right) \tilde{\Phi}_{1}\right], \\
\tilde{g}_{ \pm 1}^{(\alpha)}=\frac{1}{1 \mp \Omega_{\operatorname{tr} \alpha}}\left[ \pm i \frac{\Omega_{\mathrm{d} \alpha}}{2} \tilde{g}_{0}^{(\alpha)}+J_{0 \alpha} \frac{e_{\alpha}}{T_{\alpha}} F_{M_{\alpha}}\left(1 \mp \Omega_{* \alpha}\right) \tilde{\Phi}_{ \pm 1}\right],
\end{gathered}
$$

ou, de forma alternativa, em componentes senoidais e cossenoidais,

$$
\begin{aligned}
& \tilde{g}_{\alpha 0}=\frac{e_{\alpha} F_{M_{\alpha}}}{T_{\alpha}} J_{0 \alpha}\left[\left(1+\frac{1}{2} \frac{\Omega_{\mathrm{d} \alpha}^{2}}{1-\Omega_{\operatorname{tr} \alpha}^{2}}\right) \tilde{\Phi}_{0}-\frac{1}{2}\left(\frac{\Omega_{\mathrm{d} \alpha}}{1-\Omega_{\operatorname{tr} \alpha}^{2}}-\frac{\Omega_{\mathrm{d} \alpha} \Omega_{\operatorname{tr} \alpha} \Omega_{* \alpha}}{1-\Omega_{\operatorname{tr} \alpha}^{2}}\right) \tilde{\Phi}_{s}-\right. \\
& \left.\frac{i}{2}\left(\frac{\Omega_{\mathrm{d} \alpha} \Omega_{\operatorname{tr} \alpha}}{1-\Omega_{\operatorname{tr} \alpha}^{2}}-\frac{\Omega_{\mathrm{d} \alpha} \Omega_{* \alpha}}{1-\Omega_{\operatorname{tr} \alpha}^{2}}\right) \tilde{\Phi}_{c}\right] \\
& \tilde{g}_{\alpha s}=\frac{e_{\alpha} F_{M_{\alpha}}}{T_{\alpha}} J_{0 \alpha}\left[-\frac{\Omega_{\mathrm{d} \alpha}}{1-\Omega_{\operatorname{tr} \alpha}^{2}} \tilde{\Phi}_{0}+\left(\frac{1}{1-\Omega_{\operatorname{tr} \alpha}^{2}}-\frac{\Omega_{\operatorname{tr} \alpha} \Omega_{* \alpha}}{1-\Omega_{\operatorname{tr} \alpha}^{2}}\right) \tilde{\Phi}_{s}+\right. \\
& \left.i\left(\frac{\Omega_{\operatorname{tr} \alpha}}{1-\Omega_{\operatorname{tr} \alpha}^{2}}-\frac{\Omega_{* \alpha}}{1-\Omega_{\operatorname{tr} \alpha}^{2}}\right) \tilde{\Phi}_{c}\right] \\
& \tilde{g}_{\alpha c}=\frac{e_{\alpha} F_{M_{\alpha}}}{T_{\alpha}} J_{0 \alpha}\left[i \frac{\Omega_{\mathrm{d} \alpha} \Omega_{\operatorname{tr} \alpha}}{1-\Omega_{\operatorname{tr} \alpha}^{2}} \tilde{\Phi}_{0}-i\left(\frac{\Omega_{\operatorname{tr} \alpha}}{1-\Omega_{\operatorname{tr} \alpha}^{2}}-\frac{\Omega_{* \alpha}}{1-\Omega_{\operatorname{tr} \alpha}^{2}}\right) \tilde{\Phi}_{s}+\right. \\
& \left.\left(\frac{1}{1-\Omega_{\operatorname{tr} \alpha}^{2}}-\frac{\Omega_{\operatorname{tr} \alpha} \Omega_{* \alpha}}{1-\Omega_{\operatorname{tr} \alpha}^{2}}\right) \tilde{\Phi}_{c}\right] .
\end{aligned}
$$

A partir da integração no espaço de velocidades de (4.66), (4.67) e (4.68), obtemos os elementos da matriz central, de acordo com a representação matricial em (4.32). Este cálculo, quando 
feito em detalhes, resulta nas seguintes expressões:

$$
\begin{array}{ll}
\mathcal{R}_{00}=\mathcal{L}_{0}^{(i)}+\frac{1}{2} \mathcal{L}_{2}^{(i)}-1, & \mathcal{R}_{s s}=\mathcal{R}_{c c}=\mathcal{L}_{0}^{(i)}-\mathcal{L}_{011}^{(i)}-1-\tau_{i} \\
\mathcal{R}_{0 s}=-\frac{1}{2} \mathcal{L}_{1}^{(i)}+\frac{1}{2} \mathcal{L}_{111}^{(i)}, & \mathcal{R}_{s 0}=-\mathcal{L}_{1}^{(i)}, \quad \mathcal{R}_{s c}=-\mathcal{R}_{c s}=i \mathcal{L}_{01}^{(i)}-i \mathcal{L}_{001}^{(i)}, \\
\mathcal{R}_{0 c}=-\frac{i}{2} \mathcal{L}_{11}^{(i)}+\frac{i}{2} \mathcal{L}_{101}^{(i)}, & \mathcal{R}_{c 0}=i \mathcal{L}_{11}^{(i)}
\end{array}
$$

que, com o uso de (F.36), ao separar partes reais $(\mathcal{F})$ de imaginárias $(\mathcal{K})$, podem ser desenvolvidas da seguinte forma:

$$
\begin{aligned}
& \mathcal{R}_{00}^{(\mathcal{F})}=\frac{1}{2}\left(-\Omega^{2}+\frac{7}{4}+\frac{23}{8 q^{2} \Omega^{2}}+\frac{9}{8 q^{4} \Omega^{4}}\right) \frac{\mathrm{k}_{r}^{2} \rho_{i}^{2}}{\Omega^{2}} \\
& \mathcal{R}_{00}^{(\mathcal{K})}=-i \frac{\sqrt{\pi}}{2}\left(1+\frac{1}{q^{2} \Omega^{2}}+\frac{1}{2 q^{4} \Omega^{4}}\right) \frac{\mathrm{k}_{r}^{2} \rho_{i}^{2}}{\Omega^{2}} q^{5} \Omega^{5} \mathrm{e}^{-q^{2} \Omega^{2}}, \\
& \mathcal{R}_{0 s}^{(\mathcal{F})}=-\frac{1}{2}\left(1+\frac{1}{q^{2} \Omega^{2}}+\frac{9}{4 q^{4} \Omega^{4}}\right) \frac{\mathrm{k}_{r} \rho_{i}}{\Omega}+ \\
& \mathcal{R}_{0 s}^{(\mathcal{K})}=i \frac{\sqrt{\pi}}{2}\left[1+\frac{1}{2 q^{2} \Omega^{2}}-\left(\eta_{i} q^{2} \Omega^{2}+1+\frac{1+\eta_{i} / 2}{2 q^{2} \Omega^{2}}\right) \Omega_{* i}\right] \frac{\mathrm{k}_{r} \rho_{i}}{\Omega} q^{3} \Omega^{3} \mathrm{e}^{-q^{2} \Omega^{2}} \\
& \mathcal{R}_{0 c}^{(\mathcal{F})}=-\frac{\sqrt{\pi}}{2}\left[\frac{1}{q^{2} \Omega^{2}}+\frac{1}{q^{4} \Omega^{4}}-\left(\eta_{i}+\frac{1}{q^{2} \Omega^{2}}+\frac{1+\eta_{i} / 2}{2 q^{4} \Omega^{4}}\right) \Omega_{* i}\right] \frac{\mathrm{k}_{r} \rho_{i}}{\Omega} q^{5} \Omega^{5} \mathrm{e}^{-q^{2} \Omega^{2}}, \\
& \mathcal{R}_{0 c}^{(\mathcal{K})}=\frac{i}{2}\left(1+\eta_{i}+\frac{1+2 \eta_{i}}{q^{2} \Omega^{2}}+\frac{9+3 \eta_{i} / 4}{4 q^{4} \Omega^{4}}\right) \frac{\mathrm{k}_{r} \rho_{i}}{\Omega} \Omega_{* i} \\
& \mathcal{R}_{s 0}^{(\mathcal{F})}=-\left(1+\frac{1}{q^{2} \Omega^{2}}+\frac{9}{4 q^{4} \Omega^{4}}\right) \frac{\mathrm{k}_{r} \rho_{i}}{\Omega}, \quad \mathcal{R}_{s 0}^{(\mathcal{K})}=i \sqrt{\pi}\left(1+\frac{1}{q^{2} \Omega^{2}}\right) \frac{\mathrm{k}_{r} \rho_{i}}{\Omega} q^{3} \Omega^{3} \mathrm{e}^{-q^{2} \Omega^{2}} \\
& \mathcal{R}_{s s}^{(\mathcal{F})}=-\tau_{i}+\frac{1}{2 q^{2} \Omega^{2}}+\frac{3}{4 q^{4} \Omega^{4}}, \quad \mathcal{R}_{s s}^{(\mathcal{K})}=-i \sqrt{\pi}\left[-\frac{1}{q^{2} \Omega^{2}}+\left(\eta_{i}+\frac{1-\eta_{i}}{2 q^{2} \Omega^{2}}\right) \Omega_{* i}\right] q^{3} \Omega^{3} \mathrm{e}^{-q^{2} \Omega^{2}}, \\
& \mathcal{R}_{c 0}^{(\mathcal{F})}=\sqrt{\pi}\left(1+\frac{1}{2 q^{2} \Omega^{2}}\right) \frac{\mathrm{k}_{r} \rho_{i}}{\Omega} q^{3} \Omega^{3} \mathrm{e}^{-q^{2} \Omega^{2}}
\end{aligned}
$$

onde $\mathcal{R}_{a b}=\mathcal{R}_{a b}^{(\mathcal{F})}+\mathcal{R}_{a b}^{(\mathcal{K})}(a, b \rightarrow 0, s, c)$.

Em ordem dominante, desconsiderando termos quadráticos da função exponencial, o deter- 
minante da matriz central em (4.32), o qual pode ser aproximado por

$$
\mathcal{R}_{s s}\left(\mathcal{R}_{s s} \mathcal{R}_{00}-\mathcal{R}_{0 s} \mathcal{R}_{s 0}-\mathcal{R}_{0 c} \mathcal{R}_{c 0}\right)=0,
$$

ao ser desenvolvido, resulta na seguinte relação de dispersão:

$$
\begin{aligned}
& \mathcal{F}(\Omega)+i \mathcal{K}(\Omega)=0, \quad \mathcal{F}(\Omega) \approx \sum_{j=0}^{3} C_{2 j}^{(\mathcal{F})} \Omega^{2 j}, \quad \mathcal{K}(\Omega) \approx \sqrt{\pi} \Omega^{5} \sum_{j=0}^{4} C_{j}^{(\mathcal{K})} \Omega^{j} \mathrm{e}^{-q^{2} \Omega^{2}}, \\
& C_{0}^{(\mathcal{F})}=\left(\Omega_{\mathrm{s} 2}^{2}+\Omega_{*_{\mathrm{s}}}^{2}\right) \Omega_{\mathrm{s} 0}^{2}, \quad C_{2}^{(\mathcal{F})}=2 \Omega_{\mathrm{g} 0}^{2} \Omega_{\mathrm{s} 0}^{2}+\Omega_{\mathrm{s} 1}+\Omega_{*_{\mathrm{d}}}^{2}, \quad C_{4}^{(\mathcal{F})}=-\left(\Omega_{\mathrm{g} 0}^{2}+2 \Omega_{\mathrm{s} 0}^{2}+\Omega_{* e}^{2}\right), \\
& C_{6}^{(\mathcal{F})}=1, \quad C_{0}^{(\mathcal{K})}=\left(3 \eta_{i}-2\right) \eta_{i} \Omega_{* i} \Omega_{* e} \Omega_{\mathrm{s} 0}^{2}, \quad C_{1}^{(\mathcal{K})}=\left(1+\tau_{e}\right)\left(3 \eta_{i}-2\right) \Omega_{* i} \Omega_{\mathrm{s} 0}^{2}, \\
& C_{2}^{(\mathcal{K})}=2\left(1+\tau_{i}\right) \Omega_{\mathrm{s} 0}^{2}+\left(\eta_{i}-1\right) \Omega_{* e}^{2}, \quad C_{3}^{(\mathcal{K})}=-1, \quad C_{4}^{(\mathcal{K})}=1 .
\end{aligned}
$$

As frequências utilizadas em (4.77) são definidas na tabela 4.1, a qual também mostra os valores aproximados destas frequências no centro e na borda da coluna de plasma. 
Tabela 4.1: Frequências típicas normalizadas (por $v_{T_{i}} / R_{0}$ ) relacionadas a efeitos geodésicos, acústicos de íons e diamagnéticos.

\begin{tabular}{|c|l|c|c|}
\hline Frequência & Expressão analítica & $\tau_{e}=1, q=3.5$ (borda) & $\tau_{e}=3, q=2$ (centro) \\
\hline$\Omega_{\mathrm{g} 0}^{2}$ & $\frac{7}{4}+\tau_{e}$ & 2.75 & 4.75 \\
\hline$\Omega_{\mathrm{s} 0}^{2}$ & $\frac{\tau_{e}}{2 q^{2}}$ & $2.04 \times 10^{-2}$ & $1.88 \times 10^{-1}$ \\
\hline$\Omega_{\mathrm{s} 1}^{2}$ & $\left(\frac{23}{4} \tau_{i}+4+\tau_{e}\right) \Omega_{\mathrm{s} 0}^{2}$ & $2.19 \times 10^{-1}$ & 1.68 \\
\hline$\Omega_{* \mathrm{~g}}^{2}$ & $\left(1+\tau_{e}+\eta_{i}\right) \Omega_{* e}^{2}$ & $\left(1+0.5 \eta_{i}\right) \frac{\rho_{i}^{2} / L_{N}^{2}}{R_{0}^{2} / r^{2}}$ & $\left(2+0.5 \eta_{i}\right) \frac{\rho_{i}^{2} / L_{N}^{2}}{R_{0}^{2} / r^{2}}$ \\
\hline$\Omega_{* \mathrm{~d}}^{2}$ & $\left(\frac{3}{4}-\eta_{i}\right) \Omega_{* e}^{2}$ & \\
\hline$\Omega_{\mathrm{s} 2}^{2}$ & $\left(\frac{15}{2} \tau_{i}+\frac{9}{4}\right) \Omega_{\mathrm{s} 0}^{4}$ & & \\
\hline$\Omega_{* \mathrm{~s}}^{2}$ & $\tau_{i}\left(\eta_{i}^{2}+\frac{9}{2} \eta_{i}-\frac{17}{4}\right) \Omega_{* e}^{2}$ & & \\
\hline & & \\
\hline
\end{tabular}

\subsubsection{Soluções no limite de fluido}

O limite de fluido é obtido considerando $\mathrm{e}^{-q^{2} \Omega^{2}} \rightarrow 0$ em (4.77), ou seja, através da resolução de $\mathcal{F}(\Omega)=0$. Por se tratar de uma equação bi-cúbica e, portanto, difícil de ser solucionada analiticamente, utilizamos a forma a aproximada desta equação, a qual fornece as soluções assintóticas, considerando que as três soluções possuem as seguintes ordens de grandeza: $\Omega_{1} \sim$ $\Omega_{\mathrm{g} 0} \sim 1, \Omega_{2} \sim \Omega_{\mathrm{s} 0} \sim \delta \ll 1$ e $\Omega_{3} \sim \Omega_{*_{\mathrm{e}}} \sim \delta \ll 1$

- Primeira solução (GAM): Por se tratar da frequência de valor mais alto, $\Omega_{1} \sim 1$, podemos desprezar $C_{0}^{(\mathcal{F})}=\mathcal{O}\left(\delta^{4}\right)$. de forma que $\mathcal{F}=0$ torna-se uma equação quadrática em $\Omega^{2}$ cuja solução positiva fornece como solução a frequência dos GAM corrigida pelo 
fator de segurança $(q)$ e por efeitos diamagnéticos:

$$
\Omega_{\mathrm{GAM}}^{2}=\Omega_{\mathrm{g} 0}^{2}+\frac{\Omega_{\mathrm{s} 1}^{2}}{\Omega_{\mathrm{g} 0}^{2}}+\frac{\Omega_{* \mathrm{~g}}^{2}}{\Omega_{\mathrm{g} 0}^{2}}
$$

- Segunda solução (SWs): Neste caso, como a solução é $\Omega_{2} \sim \delta$, o termo $\Omega^{6}$ pode ser desprezado em (4.77). Admitindo a condição $\Omega_{\mathrm{s} 0} \gg \Omega_{* e}$ obtemos assintoticamente a frequência de SWs corrigida por efeitos diamagnéticos:

$$
\Omega_{\mathrm{SW}}^{2}=\left(1+\frac{7}{4} \tau_{i}^{2}\right) \Omega_{\mathrm{s} 0}^{2}-\left[\eta_{i}^{2}+\left(\frac{1}{2}+\tau_{e}\right) \eta_{i}-\frac{3}{4}\left(\frac{5}{3}+\tau_{e}\right)\right] \Omega_{* i}^{2}
$$

- Terceira solução (efeito diamagnético): Esta solução corresponde ao caso em que $\Omega_{* e} \gg \Omega_{\mathrm{s} 0}$ e, nos regimes em que $\eta_{i} \gg 3 / 4$ ou $\eta_{i} \ll 3 / 4$ ela pode ser aproximada por:

$$
\Omega_{\mathrm{dia}}^{2}=\frac{\Omega_{*_{\mathrm{d}}}^{2}}{\Omega_{\mathrm{g} 0}^{2}}+f_{\text {dia }}\left(\eta_{i}, \tau_{e}\right) \frac{\Omega_{\mathrm{s} 0}^{2}}{\Omega_{\mathrm{g} 0}^{2}}
$$

onde

$$
f\left(\eta_{i}, \tau_{e}\right)=\left(\eta_{i}-\frac{3}{4}\right)^{-1}\left[\Omega_{\mathrm{g} 0}^{4} \eta_{i}^{2}+\left(\Omega_{\mathrm{g} 0}^{4}+\frac{\Omega_{\mathrm{g} 0}^{2}}{2}-\frac{29}{16}\right) \eta_{i}-\left(\frac{3}{4} \Omega_{\mathrm{g} 0}^{4}+\frac{5}{4} \Omega_{\mathrm{g} 0}^{2}-\frac{87}{64}\right)\right]
$$

Com relação aos limites assintóticos de $f_{\text {dia }}$, há dois casos a ser considerados:

- Regime de fraco gradiente de temperatura $\left(\eta_{i} \ll 1\right)$ : A solução é estável e pode ser aproximada por

$$
\Omega_{\mathrm{dia}}=\frac{3}{4} \frac{\Omega_{* e}^{2}}{\Omega_{\mathrm{g} 0}^{2}}+\left(\Omega_{\mathrm{g} 0}^{4}+\frac{5}{3} \Omega_{\mathrm{g} 0}^{2}-\frac{29}{16}\right) \frac{\Omega_{\mathrm{s} 0}^{2}}{\Omega_{\mathrm{g} 0}^{2}}
$$

- Regime de forte gradiente de temperatura $\left(\eta_{i} \gg 1\right)$ : Este caso se caracteriza por ser instável e não oscilatório se ocorrer fortes gradientes de densidade, ou seja, se $\Omega_{* e} \gtrsim \sqrt{2} \Omega_{\mathrm{g} 0} / 2 q$. A taxa de crescimento desta instabilidade pode ser aproximada 


$$
\Gamma_{\text {inst }}=\sqrt{\eta_{i}}\left(\frac{\Omega_{* e}^{2}}{\Omega_{\mathrm{g} 0}^{2}}-\tau_{i} \Omega_{\mathrm{s} 0}^{2}\right)^{1 / 2}
$$

\subsubsection{Efeito cinético em modos geodésicos (amortecimento de Landau)}

Para o cálculo da taxa de amortecimento das soluções mostradas em (4.78), (4.79) e (4.82), utilizamos as expressões (4.56) e (4.77), o que resulta nos seguintes valores:

$$
\begin{aligned}
& \Gamma_{\mathrm{GAM}}=-q^{5} \Omega_{\mathrm{g} 0}^{4} \frac{\sqrt{\pi}}{2}\left[1+\left(5 \Omega_{\mathrm{g} 0}^{2}-\frac{9}{2}+\frac{29 / 16}{\Omega_{\mathrm{g} 0}^{2}}\right) \frac{\Omega_{\mathrm{s} 0}^{2}}{\Omega_{\mathrm{g} 0}^{2}}+\eta_{i} \frac{\Omega_{* e}}{\Omega_{\mathrm{g} 0}}+\right. \\
& \left.\left(1+\eta_{i}-\frac{\Omega_{* \mathrm{~d}}^{2}}{\Omega_{\mathrm{g} 0}^{2}}\right) \frac{\Omega_{* e}^{2}}{\Omega_{\mathrm{g} 0}^{2}}\right] \mathrm{e}^{-q^{2} \Omega_{\mathrm{GAM}}^{2}}
\end{aligned}
$$

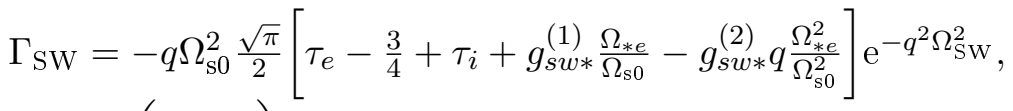

$$
\begin{aligned}
& g_{s w *}^{(1)}=\left(\frac{\eta_{i}}{2}-1\right) \tau_{e}+3-\frac{5}{4} \eta_{i}+\frac{17 \eta_{i}-57}{8} \tau_{i}, \quad g_{s w *}^{(2)}=\frac{\eta_{i}-1}{4} \tau_{e}+\frac{3}{4} \eta_{i}^{2}-\frac{3}{2} \eta_{i}+1, \\
& \Gamma_{\mathrm{dia}}=-\left(1+\tau_{e}\right) \frac{\sqrt{\pi}}{2} \frac{\Omega_{* e}^{4}}{\Omega_{\mathrm{g} 0}^{4}}\left[\left(\frac{3}{4} \frac{\Omega_{* e}}{\Omega_{\mathrm{g} 0}^{2}}\right)^{2}-\frac{3}{2}\left(\Omega_{\mathrm{g} 0}^{2}-\sqrt{3} \Omega_{\mathrm{g} 0}-\frac{55}{24}-\frac{87}{32 \Omega_{\mathrm{g} 0}^{2}}\right) \tau_{i} \frac{\Omega_{\mathrm{s} 0}^{2}}{\Omega_{\mathrm{g} 0}^{2}}\right] \mathrm{e}^{-q^{2} \Omega_{\mathrm{dia}}^{2}} .
\end{aligned}
$$

\subsection{Sumário e discussão}

Neste capítulo, mostramos, essencialmente, a importância da aplicação do modelo girocinético ao estudo de modos de baixas frequências. A partir deste modelo, inicialmente apresentado no capítulo 2, inúmeros fenômenos que ocorrem em modos de baixas frequências podem ser analisados, entre eles: efeitos diamagnéticos, amortecimento de Landau, efeitos eletromagnéticos, efeitos de partículas aprisionadas e automodos relacionados a modos Alfvenicos e a GAM. O maior destaque deste modelo, no entando, se deve à possibilidade de obter a taxa de amortecimento não colisional (amortecimento de Landau) e de investigar efeitos de partículas aprisionadas em modos geodésicos, o que não é possível a partir de modelos de fluidos por se 
tratar de efeitos puramente cinéticos.

Quanto à metodologia adotada neste capítulo, com a finalidade de elucidar a utilização do modelo girocinético, dividimos este capítulo em etapas. Primeiramente consideramos $k_{\|} v_{T_{i}}=0$, $k_{\|} v_{T_{e}} \rightarrow \infty$ e $\nabla F_{M_{\alpha}}=0$, o que torna o cálculo analítico consideravelmente mais simples e permite obter os termos essenciais da frequência de GAM. Com estas considerações é possível entender mais facilmente a dinâmica básica de GAM pelo modelo girocinético. Em seguida, considerando $k_{\|} v_{T_{i}}$ finito, porém não levando em conta ainda o amortecimento de Landau, o qual é importante para baixos valores de $q$, além de correções de ordem $q^{-2}$ para a frequência dos GAM, também obtivemos a solução para $S W s$. Observamos, nesta parte, que a frequência $\omega=\sqrt{T_{e} / m_{i} q^{2} R_{0}^{2}}$, representa uma singularidade, pois os denominadores da relação de dispersão e da componente seno do potencial eletrostático se anulam nesta frequência. O amortecimento de Landau é então considerado de forma aproximada (com objetivos didáticos) na subseção 4.1.3. Na seção 4.3 discutimos brevemente a forma mais geral da equação girocinética, a qual permite a análise de efeitos diamagnéticos além de outros efeitos em modos geodésicos e Alfvenicos, os quais pretendemos estudar em trabalhos futuros. Na última seção, obtivemos as expressões analíticas para a frequência de GAM e SWs levando em conta correções assintóticas decorrentes de gradientes radiais de densidade e temperatura de íons, ou seja, $\nabla F_{M_{\alpha}} \neq 0$ é considerado. Taxas de amortecimento correspondentes a estas frequências, as quais são importantes quando $k_{\|} v_{T_{i}} \lesssim v_{T_{i}} / R_{0}$ também foram calculadas nesta seção.

As expressões analíticas para a frequência dos GAM que obtivemos ao considerar amortecimento de Landau concordam com os valores obtidos em trabalhos anteriores [35, 66, 82, 85]. Apenas no limite de íons frios, $T_{i} \ll T_{e}$, considerando elétrons no regime adiabático e isotérmico $\left(\gamma_{e}=1\right)$, o que é razoável para tokamaks [86], a expressão cinética para a frequência de GAM concorda com a expressão obtida pelo modelo da MHD ideal [26,34]. Conclui-se, portanto, que a dinâmica de elétrons não é influenciada por efeitos cinéticos e pode ser bem descrita pela teoria de fluidos. A correção devido ao efeito da anisotropia da pressão perturbada para íons, com relação às componentes perpendicular e paralela, é da ordem de $5 \%$ e, portanto não desempenha um papel muito significativo no valor da frequência dos GAM.

As expressões analíticas para a frequência de GAM e SWs foram analisadas nos limites 
assintóticos de maior interesse em cada caso. A solução que corresponde a GAM na presença de efeitos diamagnéticos, de acordo com (4.78), tem sua frequência aumentada devido ao gradiente de densidade e de temperatura. Este fato pode ser utilizado, em conjunto com a investigação de outros efeitos em GAM, para explicar e possivelmente identificar alguns tipos de modos MHD detectados recentemente no TCABR [87]. No limite $T_{e} \gg T_{i}$ e $1 \ll q \ll q_{\max }$, que normalmente ocorre em posições relativamente próximas do centro da coluna de plasma, a expressão (4.79) pode ser utilizada para o cálculo aproximado da frequência de SWs, desde que não ocorram fortes gradientes de densidade. No extremo oposto, quando $q \sim q_{\max }$ e há fortes gradientes de densidade, a solução correspondente a SWs é afetada por efeitos diamagnéticos e, neste caso, a expressão (4.80) deve ser utilizada para o cálculo da frequência. Para o caso específico em que $\eta_{i} \gg 3 / 4$, cuja solução é mostrada em (4.83), estes modos (SWs) se tornam instáveis na presença de fortes gradientes de densidade e de temperatura. É possível concluir que a estabilidade deste tipo de modo geodésico depende, não somente da relação entre os gradientes de densidade e de temperatura de íons, mas também da relação entre o fator de segurança e o gradiente de densidade. Esta última condição pode ser utilizada em experimentos para a obtenção do perfil radial de $q$ a partir de medidas da frequência deste tipo de modo.

Em trabalhos futuros pretendemos estudar efeitos eletromagnéticos causados por perturbações do campo magnético perpendicular (em relação ao campo magnético de equilíbrio), conforme investigado anteriormente em [64,82]. Trata-se de um modelo cujos cálculos são mais extensos principalmente devido à necessidade de incluir modos toroidais $n \neq 0$ e modos poloidais $m= \pm 2$, conforme discutido em [68]. É interessante considerar simultaneamente efeitos eletromagnéticos e efeitos diamagnéticos em GAM e BAEs (Beta-induced Alfvén Eigenmodes), conforme discutido em [82], pois outras correções para a frequência de modos geodésicos são necessárias para a confirmação experimental destes modos [87]. Além disso, ao considerar efeitos eletromagnéticos, os quais estão relacionados à dinâmica de elétrons, o efeito de gradientes de temperatura de elétrons pode ser quantificado, conforme discutido no capítulo 3. 


\section{Capítulo 5}

\section{Conclusões e direções futuras}

Neste capítulo, apresentamos, primeiramente, uma discussão sobre os modelos de fluido e girocinético e conclusões sobre os resultados obtidos quanto à abrangência e validade destes modelos e quanto à aplicação destes retultados no tokamak. Em seguida, opções para o desenvolvimento de modelos mais abrangentes são discutidas e propostas para trabalhos futuros são apresentadas.

\subsection{Modelo de fluidos}

No modelo de fluidos partimos das equações de Braginskii que descrevem a evolução das grandezas macroscópicas do plasma: pressão $(p)$, viscosidade paralela $\left(\pi_{\|}\right)$, densidade $(n)$ e velocidade (v), as quais são medidas por meio de diagnósticos no tokamak, para estudar equilíbrio MHD com rotação poloidal e toroidal e modos geodésicos de baixas frequências na presença de rotação de equilíbrio e gradientes de densidade e temperatura. Pelo fato de tratar-se de um modelo mais acessível para a compreensão física dos fenômenos envolvidos e por permitir um tratamento mais simples de modelos não lineares, a teoria de fluidos possui importantes aplicações em física de plasmas. Os modelos considerados e a metodologia utilizada foram baseados principalmente em dois trabalhos anteriores: $[36,50]$.

Ao considerarmos equilíbrio com rotação poloidal e toroidal e o termo de fluxo de calor no modelo da MHD ideal, obtivemos correções analíticas em termos do número de Mach poloidal 
e toroidal para a frequência de fluxos zonais (ZFs), ondas de som (SWs) e modos acústicos geodésicos (GAMs). Observamos um aumento no valor da frequência destes modos, que é devido à rotação do plasma. Quando há perturbações eletrostáticas no plasma $(\tilde{\Phi} \neq 0)$, no contexto da teoria da MHD ideal, SWs só ocorrem quando há rotação poloidal de equilíbrio. Entretanto, a partir do modelo girocinético mostramos que há SWs mesmo quando rotação poloidal não é considerada.

Ao contrário do que ocorre com GAMs e SWs, que são modos acústicos e, portanto, compressíveis, observa-se que ZFs (modos incompressíveis) são fortemente influenciados pela componente binormal (perpendicular ao campo magnético de equilíbrio e normal às superfícies magnéticas) do fluxo de calor, a qual depende do gradiente radial de temperatura. Ao considerarmos fluxo de calor nas equações da MHD ideal obtivemos a expressão analítica para a frequência dos ZFs, a qual torna-se não nula devido ao efeito do fluxo de calor e da rotação poloidal de equilíbrio [76].

Ao observarmos que no regime isométrico com rotação unicamente toroidal os ZFs são instáveis [27], observamos a necessidade de considerar também rotação poloidal neste regime, o que, pelo fato de levar a contas extensas e fugir do escopo desta tese, pretendemos fazer em um trabalho futuro. A análise dos resultado produzidos por este trabalho, em conjunto com a análise do modelo de dois fluidos, no qual observamos que gradientes de temperatura são responsáveis por modos geodésicos instáveis, é promissora pois, como a rotação poloidal está relacionada com gradientes de temperatura, poderíamos obter informações adicionais sobre o perfil radial da temperatura de íons, além de entender melhor as condições de estabilidade de ZFs, SWs e GAMs.

Com relação a análise do equilíbrio MHD, consideramos três tipos de equilíbrio: adiabático, isotérmico e isométrico. A partir do perfil radial da velocidade de rotação poloidal e toroidal obtido experimentalmente no tokamak TCABR [78], observa-se que uma mudança de regime de equilíbrio com a posição radial, do isotérmico para o isométrico, implicaria em uma mudança de sentido do gradiente de temperatura do plasma, considerando que não haja inversão de sentido do campo magnético poloidal e com base no resultado experimental obtido para a rotação poloidal no TCABR. No regime adiabático observa-se que o gradiente de temperatura é nulo e, portanto, este regime é possível apenas localmente, pois a temperatura decresce do centro para 
a borda da coluna de plasma. No que se refere a inversão de sentido da velocidade de rotação toroidal na posição $r \approx 0.7 a$, o que ainda não é bem compreendido do ponto de vista teórico, é necessário considerar outros termos no modelo da MHD ideal e no modelo de dois fluidos. Estes termos são devido a colisões, pois próximo à borda da coluna de plasma o plasma se torna mais colisional e, portanto, resistivo.

Efeitos diamagnéticos provenientes de gradientes radiais de densidade e de temperatura foram considerados no modelo de dois fluidos, no qual íons são considerados no regime de fluidos (adiabático) e elétrons, devido a sua pequena massa, são considerados simultaneamente no regime adiabático e isotérmico. Devido à enorme diferença de massa entre estas partículas, é coerente considerar viscosidade paralela apenas para íons, o que equivale a levar em conta o efeito da anisotropia de pressão: a componente perpendicular é diferente da componente paralela para a pressão perturbada de íons. Assumindo o gradiente de temperatura na mesma direção do gradiente de densidade, o que é mais razoável com relação ao que se observa em experimentos, concluímos que a instabilidade dos GAMs é proveniente do gradiente de temperatura e que o gradiente de densidade, de certa forma, tende a estabilizar o plasma. Mais precisamente, quando $L_{T_{i}}^{-1}>3 L_{N}^{-1} / 4$, ocorrem modos instáveis [77]. A instabilidade e a correção da frequência dos GAMs devido a efeitos diamagnéticos (ou efeitos de deriva) são proporcionais à frequência diamagnética de elétrons, $\omega_{* e}=T_{e} / e B r L_{N}$. Este tema está relacionado ao estudo de ondas de deriva $\left(\mathrm{DW}^{1}\right)[88]$ e modos de gradiente de temperatura de íons $\left(\mathrm{ITG}^{2}\right)$ [45].

As principais conclusões provenientes do capítulo 3 sobre modelos de fluidos podem ser resumidas como:

- Devido a curvatura geodésica do campo magnético de equilíbrio no tokamak, o que acarreta na quebra de simetria poloidal do sistema, surgem modos geodésicos no plasma que, por desempenharem um papel importante no controle de turbulência e por terem aplicações na obtenção do perfil radial de parâmetros do plasma, são dignos de uma intensa investigação teórica e experimental. Informações sobre as frequências destes modos podem ser utilizadas tanto para estudar os fenômenos físicos envolvidos como para aplicações

\footnotetext{
${ }^{1}$ Drift Waves

${ }^{2}$ Ion Temperature Gradient
} 
em diagnósticos.

- A anisotropia da pressão iônica, descrita pelo tensor de viscosidade paralela $\left(\boldsymbol{\pi}_{\|}\right)$, altera o valor do coeficiente adiabático efetivo $\left(\gamma^{(\text {efetivo })}\right)$ de $5 / 3$ para $7 / 4$, fazendo com que a frequência de GAMs tenha um pequeno aumento (menor do que 5\%).

- O fato de a rotação de equilíbrio e os gradientes de temperatura e densidade produzirem um aumento de frequência no ramo de maior frequência dos GAMs pode ser utilizado para tentar explicar e identificar modos recentemente medidos no tokamak TCABR [87].

- A investigação do efeito de rotação de equilíbrio e de efeitos diamagnéticos em ZFs requer que o fluxo de calor seja levado em conta e, portanto, gradientes de temperatura desempenham um papel fundamental na dinâmica de fluxos zonais.

- Tanto a obtenção de expressões analíticas para a frequência de modos geodésicos quando rotação de equilíbrio e/ou efeitos diamagnéticos são considerados, como o estudo de equilíbrio MHD com rotação em diferentes regimes, aliados a resultados experimentais obtidos em tokamaks, podem ser utilizados em conjunto para a obtenção dos perfis radiais da temperatura de íons e do fator de segurança, o que tem ampla aplicação para a orientação de futuros experimentos no ITER.

- O gradiente de temperatura de íons é a fonte de energia responsável pela instabilidade de GAMs e a condição para esta instabilidade, que é proporcional à frequência diamagnética de elétrons, $\omega_{* e}=T_{e} / e B r L_{N}$, e também depende do gradiente de densidade, em regiões em que $q$ é muito grande, pode ser expressa de forma assintótica $(q \rightarrow \infty)$ por: $\eta_{i}=\left(\partial \ln T_{i} / \partial r\right) /\left(\partial \ln n_{0} / \partial r\right)>3 / 4$ e, portanto, observa-se que o gradiente de densidade contribui para a estabilidade de GAMs.

\subsection{Modelo girocinético}

Do ponto de vista quantitativo, a utilização do modelo girocinético no capítulo 4 teve dois objetivos: obter expressões analíticas para as frequências de GAMs e SWs na presença de efeitos 
diamagnéticos considerando, simultaneamente, termos de $\mathcal{O}\left(q^{-2}\right)$ e investigar o amortecimento de Landau destes modos, que surge devido a interação onda-partícula e, por isso, requer um tratamento cinético. Por questões didáticas, até mesmo porque modelos cinéticos apresentam maior dificuldade de compreensão física se comparados com modelos de fluidos, descrevemos separadamente cada efeito antes de considerá-lo em conjunto.O estudo do amortecimento de Landau de modos geodésicos apresentado nesta tese foi baseado na Ref. [85] cujos resultados estão em acordo com os nossos.

Para determinar a taxa de amortecimento não colisional dos modos geodésicos em questão, conforme ilustrado na seção 4.1.3, utilizamos um método iterativo no qual, primeiramente, no limite de fluido, ou seja, não levando em conta o amortecimento de Landau, obtemos uma equação bi-cúbica cuja solução fornece as frequências destes modos. Posteriormente, estas soluções são consideradas nas expressões cinéticas, as quais são expandidas em série de Taylor, considerando, nesta expansão, a taxa de amortecimento de Landau como parâmetro de baixo valor. Para a solução analítica da equação bi-cúbica, partindo do conhecimento prévio obtido pelo modelo da MHD ideal e de dois fluidos da ordem de grandeza das soluções, desprezamos termos de menor ordem para aproximá-la por uma equação bi-quadrática. Obtivemos, assim, as três frequências nos limites assimptóticos, que correspondem, respectivamente a: GAMs de alta frequência, SWs, e GAMs de baixas frequências (instáveis sob certas condições), cujas expressões analíticas são mostradas em (4.78), (4.79) e (4.80), respectivamente [89]. Com relação ao modo instável, cujo limite de maior interesse é $q \sim q_{\max }, L_{T_{i}}^{-1} \gg L_{N}^{-1}$ e $T_{i} \ll T_{e}$, observamos que, conforme mostrado em (4.83), o gradiente de temperatura iônica aliado ao gradiente de densidade constituem fontes para a instabilidade de GAMs em regiões onde o fator de segurança é alto, ou seja, normalmente na borda da coluna de plasma. Esta informação pode ser explorada experimentalmente: a partir de medidas destas frequências podemos obter uma relação entre o valor de $q$ e de $L_{N}$ e o valor de $\eta_{i}=L_{N} / L_{T_{i}}$, ou seja, como $L_{N}$ pode ser obtido experimentalmente de maneira mais simples, podemos determinar o perfil radial da temperatura de íons e de q. Da mesma forma, como a taxa de amortecimento de Landau depende de $q$, a determinação da amplitude do potencial eletrostático associado a estes modos em função do tempo pode ser utilizada para a determinação do perfil radial de $q$. 
Além de aplicações em diagnósticos na determinação do perfil radial de $q(r)$ e de $T_{i}(r)$, a expressão cinética para a frequência de GAMs obtida no capítulo 4 pode ser utilizada na comparação e identificação de modos observados no TCABR [87] com mais precisão, pois levamos em conta termos de $\mathcal{O}\left(q^{-2}\right)$. Com relação a taxa de amortecimento, esta pode ter aplicações em fenômenos de transporte, pois é provável que haja uma relação entre taxa de amortecimento do potencial eletrostático e o aumento do transporte.

\subsection{Propostas para trabalhos futuros}

Pretendemos dar continuidade à linha de pesquisa iniciada nesta tese. Um dos tópicos não mencionado anteriormente o qual pretendemos investigar é a influência em GAMs, SWs, e ZFs da população de partículas aprisionadas no plasma. O efeito da assimetria do campo magnético em partículas, cuja velocidade paralela é relativamente baixa, faz com que estas sejam aprisionadas e, devido a movimentos de deriva magnética, estas partículas descrevem movimentos especiais conhecidos como órbitas de banana [44] que, por sua vez, são indiretamente responsáveis pelo transporte neoclássico [55,56], o qual, ainda que em menos intensidade do que o transporte anômalo, degrada o confinamento do plasma. Por outro lado, há também partículas com velocidade paralela relativamente alta, as partículas passantes (ou circulantes), que conseguem vencer o poço magnético e, por não descreverem órbitas tão espaçosas contribuem menos com o transporte. Para modos de baixas frequências, tipicamente menor do que a

frequência de circulação de íons, $\omega_{\operatorname{tr} i}=\sqrt{2 T_{i} / m_{i}} / q R_{0}$, onde $T_{i}$ e $m_{i}$ são a temperatura e a massa dos íons e $R_{0}$ é o raio maior do tokamak, a dinâmica de íons e elétrons aprisionados são relevantes e portanto devem ser levados em consideração no modelo para descrever tais modos [90-92]. O efeito de partículas aprisionadas no contínuo de ondas de Alfvén cisalhadas $\left(\mathrm{SAW}^{3}\right)$ de baixa frequência foi investigado analiticamente em [93] utilizando o modelo girocinético. De acordo com [93], para que seja possível um tratamento analítico, é necessário que haja a distinção entre partículas totalmente aprisionadas e partículas totalmente circulantes a serem consideradas no modelo. As principais referências para o estudo da relação entre modos

\footnotetext{
${ }^{3}$ Shear Alfvén Waves
} 
geodésicos e a dinâmica de partículas aprisionadas que recomendamos são [93-96]. Parte da teoria envolvida neste estudo é discutida de forma mais acessível em [30,44].

Em um momento inicial os assuntos tratados nesta tese podem ser expandidos em dois ramos: o estudo de GAMs eletromagnéticos na presença de efeitos diamagnéticos e a investigação de automodos acústicos geodésicos. Seguindo a linha de pesquisa desta tese, pretendemos investigar em paralelo estes dois assuntos e depois considerá-los em conjunto. Baseado nas Refs. [64, 82], o efeito do gradiente de temperatura de elétrons em modos geodésicos de baixas frequências, descrito pela quantidade, $\eta_{e}=L_{N} / L_{T_{e}}$, pode ser quantificado quando se considera a perturbação paralela do potencial vetor $\left(\tilde{A}_{\|} \neq 0\right)$. Neste estudo, considerar a influência de segundos harmônicos poloidais $(m= \pm 2)$ é essencial, de acordo com [97], pois efeitos eletromagnéticos atuam principalmente em elétrons que constituem a corrente paralela, a qual possui dependência em $\sin (2 \theta)$. Da mesma forma, o estudo de automodos geodésicos exige que sejam levados em contas termos relacionados a $m= \pm 2$. Automodos geodésicos ocorrem em regiões onde a frequência de GAMs (contínuo) é praticamente constante com a posição radial e, por permitir obter informações sobre o potencial eletrostático perturbado $(\tilde{\Phi}(r, t))$ e por ter aplicações diagnósticas: Espectroscopia em GAM [98,99], em especial para obter o perfil radial da temperatura de íons, possui um amplo interesse na área de fusão nuclear.

Há ainda inúmeras questões em aberto quanto aos GAM e, em particular, outros efeitos e a consideração de termos de ordem superior em GAM podem ajudar a esclarecer algumas destas questões. A inclusão de termos de $\mathcal{O}\left(\mathrm{k}_{r}^{4} \rho_{i}^{4}\right)$, por exemplo, além de necessária para o estudo de automodos geodésicos, permite a determinação da dependência radial da frequência dos GAM [25]. No modelo de fluídos esses termos de ordem superior podem ser incluídos se considerados giro-viscosidade e fluxo de calor $[37,100]$. 


\section{Apêndice A}

\section{Constantes e parâmetros do TCABR e}

\section{ITER}

\section{A.1 Constantes da Física}

Tabela A.1: Constantes físicas pertinentes a esta tese

\begin{tabular}{|l|l|l|l|}
\hline Símbolo & Grandeza física & Valor numérico & $\begin{array}{l}\text { Unidades (SI) } \\
\text { (ou ordem de grandeza) }\end{array}$ \\
\hline$m_{e}$ & Massa de repouso do elétron & 0,911 & $10^{-30} \mathrm{~kg}$ \\
\hline$m_{p}$ & Massa de repouso do próton & 1,67 & $10^{-27} \mathrm{~kg}$ \\
\hline$e$ & Carga elétrica elementar & 1,60 & $10^{-19} \mathrm{C}$ \\
\hline$\varepsilon_{0}$ & Permissividade no vácuo & 0,885 & $10^{-11} \mathrm{Fm}^{-1}$ \\
\hline$\mu_{0}$ & Permeabilidade no vácuo & 1,26 & $10^{-6} \mathrm{Hm}^{-1}$ \\
\hline$h$ & Constante de Planck & 0,66 & $10^{-26} \mathrm{Js}^{-13}$ \\
\hline$k$ & Constante de Boltzmann & 1,38 & $10^{-23} \mathrm{JK}^{-1}$ \\
\hline
\end{tabular}




\section{A.2 Parâmetros do TCABR e do ITER}

Tabela A.2: Principais parâmetros dos tokamaks TCABR e ITER

\begin{tabular}{|l|l||l|l|l|}
\hline Parâmetro & Símbolo & TCABR & ITER & Unidades \\
\hline Raio maior & $R_{0}$ & 0,61 & 6,2 & $\mathrm{~m}$ \\
\hline Raio menor & $a$ & 0,18 & 2,0 & $\mathrm{~m}$ \\
\hline Campo magnético toroidal & $B_{T}$ & 1,2 & 5,3 & $\mathrm{~T}$ \\
\hline Corrente de plasma & $I_{P}$ & 0,090 & 15 & $\mathrm{MA}$ \\
\hline Fator de segurança & $q_{a}\left(q_{95}\right)$ & 3,5 & 3,0 & - \\
\hline Densidade de elétrons & $n_{e}$ & 3 (centro) $/ 0,1(a)$ & 10,1 (média) & $10^{19} \mathrm{~m}^{-3}$ \\
\hline Temperatura de elétrons & $T_{e}$ & 0,5 (centro) $/ 0,015(a)$ & 8,8 (média) & $\mathrm{keV}$ \\
\hline
\end{tabular}

\section{A.3 Ordens de grandeza de frequências e velocidades características do TCABR}

Tabela A.3: Valor da ordem de grandeza da frequência de giração, da velocidade térmica e do raio de Larmor para o tokamak TCABR

\begin{tabular}{|l|l|l|l|l|l|}
\hline$\omega_{c_{i}}$ & $10^{8} \mathrm{rad} / \mathrm{s}$ & $v_{T_{i}}$ & $5.4 \times 10^{4} \mathrm{~m} / \mathrm{s}$ & $\rho_{i}$ & $4.5 \times 10^{-4} \mathrm{~m}$ \\
\hline$\omega_{c_{e}}$ & $2.2 \times 10^{11} \mathrm{rad} / \mathrm{s}$ & $v_{T_{e}}$ & $2.3 \times 10^{6} \mathrm{~m} / \mathrm{s}$ & $\rho_{e}$ & $1.0 \times 10^{-5} \mathrm{~m}$ \\
\hline
\end{tabular}




\section{Apêndice B}

\section{Identidades e relações vetoriais}

Neste apêndice apresentamos identidades e relações vetoriais de fundamental importância para os cálculos do apêndice D e do capítulo 3. Tais relações muitas vezes envolvem o operador gradiente, $\boldsymbol{\nabla}$, que na maioria dos casos é convenientemente escrito em termos de coordenadas quasi-toroidais. As relações e identidades aqui apresentadas podem ser encontradas em [41,101, $102]$.

\section{B.1 Identidades vetoriais}

$$
\begin{gathered}
(\boldsymbol{A} \times \mathbf{B}) \cdot \boldsymbol{C}=(\mathbf{B} \times \boldsymbol{C}) \cdot \boldsymbol{A}=(\boldsymbol{C} \times \boldsymbol{A}) \cdot \mathbf{B} \\
\boldsymbol{A} \times(\mathbf{B} \times \boldsymbol{C})=(\boldsymbol{A} \cdot \boldsymbol{C}) \mathbf{B}-(\boldsymbol{A} \cdot \mathbf{B}) \boldsymbol{C} \\
(\boldsymbol{A} \times \mathbf{B}) \cdot(\boldsymbol{C} \times \boldsymbol{D})=(\boldsymbol{A} \cdot \boldsymbol{C})(\mathbf{B} \cdot \boldsymbol{D})-(\boldsymbol{A} \cdot \boldsymbol{D})(\mathbf{B} \cdot \boldsymbol{C})
\end{gathered}
$$


B.2 Identidades e teoremas fundamentais

$$
\begin{gathered}
\boldsymbol{\nabla} \cdot(\boldsymbol{\nabla} \times \boldsymbol{A})=0, \quad \boldsymbol{\nabla} \times(\boldsymbol{\nabla} f)=0 \\
\int_{V} \boldsymbol{\nabla} \cdot \boldsymbol{A} d V=\oint_{S} \boldsymbol{A} \cdot d \boldsymbol{S}, \quad \int_{S}(\boldsymbol{\nabla} \times \boldsymbol{A}) \cdot d \boldsymbol{S}=\oint_{l} \boldsymbol{A} \cdot d \boldsymbol{l}
\end{gathered}
$$

B.3 Identidades envolvendo o operador $\nabla$

$$
\begin{aligned}
& \boldsymbol{\nabla} \cdot(f \boldsymbol{A})=f \boldsymbol{\nabla} \cdot \boldsymbol{A}+\boldsymbol{A} \cdot \boldsymbol{\nabla} f \\
& \boldsymbol{\nabla} \times(f \boldsymbol{A})=f \boldsymbol{\nabla} \times \boldsymbol{A}+\boldsymbol{\nabla} f \times \boldsymbol{A} \\
& \boldsymbol{\nabla} \cdot(\boldsymbol{A} \times \mathbf{B})=(\boldsymbol{\nabla} \times \boldsymbol{A}) \cdot \mathbf{B}-(\boldsymbol{\nabla} \times \mathbf{B}) \cdot \boldsymbol{A} \\
& \boldsymbol{\nabla} \times(\boldsymbol{A} \times \mathbf{B})=(\boldsymbol{\nabla} \cdot \mathbf{B}) \boldsymbol{A}-(\boldsymbol{\nabla} \cdot \boldsymbol{A}) \mathbf{B}+(\mathbf{B} \cdot \boldsymbol{\nabla}) \boldsymbol{A}-(\boldsymbol{A} \cdot \boldsymbol{\nabla}) \mathbf{B} \\
& \boldsymbol{A} \times(\boldsymbol{\nabla} \times \mathbf{B})+\mathbf{B} \times(\boldsymbol{\nabla} \times \boldsymbol{A})=\boldsymbol{\nabla}(\boldsymbol{A} \cdot \mathbf{B})-(\boldsymbol{A} \cdot \boldsymbol{\nabla}) \mathbf{B}-(\mathbf{B} \cdot \boldsymbol{\nabla}) \boldsymbol{A} \\
& \boldsymbol{\nabla} \times(\boldsymbol{\nabla} \times \boldsymbol{A})=\boldsymbol{\nabla}(\boldsymbol{\nabla} \cdot \boldsymbol{A})-\nabla^{2} \boldsymbol{A} \\
& \boldsymbol{\nabla} \cdot(\boldsymbol{A B})=(\boldsymbol{\nabla} \cdot \boldsymbol{A}) \mathbf{B}+(\boldsymbol{A} \cdot \boldsymbol{\nabla}) \mathbf{B}
\end{aligned}
$$


B.4 Gradiente, Divergente, Rotacional e Laplaciano em coordenadas cilíndricas

$$
\begin{gathered}
\nabla \Psi=\frac{\partial \Psi}{\partial R} \hat{\mathbf{e}}_{R}+\frac{1}{R} \frac{\partial \Psi}{\partial \varphi} \hat{\mathbf{e}}_{\varphi}+\frac{\partial \Psi}{\partial Z} \hat{\mathbf{e}}_{Z}, \quad R=R_{0}+r \cos \theta \\
\nabla \cdot \mathbf{B}=\frac{1}{R} \frac{\partial\left(R B_{R}\right)}{\partial R}+\frac{1}{R} \frac{\partial B_{\varphi}}{\partial \varphi}+\frac{\partial B_{Z}}{\partial Z} \\
\boldsymbol{\nabla} \times \mathbf{B}=\left[\frac{1}{R} \frac{\partial B_{Z}}{\partial \varphi}-\frac{\partial B_{\varphi}}{\partial Z}\right] \hat{\mathbf{e}}_{R}+\left[\frac{\partial B_{R}}{\partial Z}-\frac{\partial B_{Z}}{\partial R}\right] \hat{\mathbf{e}}_{\varphi}+\frac{1}{R}\left[\frac{\partial\left(R B_{\varphi}\right)}{\partial R}-\frac{\partial B_{R}}{\partial \varphi}\right] \hat{\mathbf{e}}_{Z} \\
\nabla^{2} \Psi=\frac{1}{R} \frac{\partial}{\partial R}\left(R \frac{\partial \Psi}{\partial R}\right)+\frac{1}{R^{2}} \frac{\partial^{2} \Psi}{\partial \varphi^{2}}+\frac{\partial^{2} \Psi}{\partial Z^{2}}
\end{gathered}
$$

B.5 Gradiente, Divergente e Rotacional em coordenadas quasi-toroidais

$$
\begin{gathered}
\nabla \Psi=\frac{\partial \Psi}{\partial r} \hat{\mathbf{e}}_{r}+\frac{1}{r} \frac{\partial \Psi}{\partial \theta} \hat{\mathbf{e}}_{\theta}+\frac{1}{R} \frac{\partial \Psi}{\partial \phi} \hat{\mathbf{e}}_{\phi}, \\
\boldsymbol{\nabla} \cdot \mathbf{B}=\frac{1}{R r}\left[\frac{\partial}{\partial r}\left(R r B_{r}\right)+\frac{\partial}{\partial \theta}\left(R B_{\theta}\right)+r \frac{\partial B_{\phi}}{\partial \phi}\right] \mathrm{e}
\end{gathered}
$$




$$
\begin{gathered}
\frac{1}{R}\left[\left(\frac{1}{r} \frac{\partial\left(R B_{\phi}\right)}{\partial \theta}-\frac{\partial B_{\theta}}{\partial \phi}\right) \hat{\mathbf{e}}_{r}+\left(\frac{\partial B_{r}}{\partial \phi}-\frac{\partial\left(R B_{\phi}\right)}{\partial r}\right) \hat{\mathbf{e}}_{\theta}+\frac{R}{r}\left(\frac{\partial\left(r B_{\theta}\right)}{\partial r}-\frac{\partial B_{r}}{\partial \theta}\right) \hat{\mathbf{e}}_{\phi}\right] \\
\nabla^{2} \Psi=\frac{1}{R r}\left[\frac{\partial}{\partial r}\left(R r \frac{\partial \Psi}{\partial r}\right)+\frac{\partial}{\partial \theta}\left(\frac{R}{r} \frac{\partial \Psi}{\partial \theta}\right)+\frac{\partial}{\partial \phi}\left(\frac{r}{R} \frac{\partial \Psi}{\partial \phi}\right)\right]
\end{gathered}
$$

\section{B.6 Derivadas de versores em coordenadas cilíndricas}

$$
\begin{gathered}
\frac{\partial \hat{\mathbf{e}}_{R}}{\partial R}=\frac{\partial \hat{\mathbf{e}}_{\varphi}}{\partial R}=\frac{\partial \hat{\mathbf{e}}_{R}}{\partial R}=0, \quad \frac{\partial \hat{\mathbf{e}}_{Z}}{\partial \varphi}=0, \quad \frac{\partial \hat{\mathbf{e}}_{R}}{\partial Z}=\frac{\partial \hat{\mathbf{e}}_{\varphi}}{\partial Z}=\frac{\partial \hat{\mathbf{e}}_{Z}}{\partial Z}=0 \\
\frac{\partial \hat{\mathbf{e}}_{R}}{\partial \varphi}=\hat{\mathbf{e}}_{\varphi}, \quad \frac{\partial \hat{\mathbf{e}}_{\varphi}}{\partial \varphi}=-\hat{\mathbf{e}}_{R} .
\end{gathered}
$$

\section{B.7 Derivadas de versores em coordenadas quasi-toroidais}

$$
\begin{gathered}
\frac{\partial \hat{\mathbf{e}}_{r}}{\partial r}=\frac{\partial \hat{\mathbf{e}}_{\theta}}{\partial r}=\frac{\partial \hat{\mathbf{e}}_{\phi}}{\partial r}=0, \quad \frac{\partial \hat{\mathbf{e}}_{\phi}}{\partial \theta}=0, \\
\frac{\partial \hat{\mathbf{e}}_{r}}{\partial \theta}=\hat{\mathbf{e}}_{\theta}, \quad \frac{\partial \hat{\mathbf{e}}_{\theta}}{\partial \theta}=-\hat{\mathbf{e}}_{r}, \\
\frac{\partial \hat{\mathbf{e}}_{r}}{\partial \phi}=-\cos \theta \hat{\mathbf{e}}_{\phi}, \quad \frac{\partial \hat{\mathbf{e}}_{\theta}}{\partial \phi}=-\sin \theta \hat{\mathbf{e}}_{\phi}, \quad \frac{\partial \hat{\mathbf{e}}_{\phi}}{\partial \phi}=-\cos \theta \hat{\mathbf{e}}_{r}+\sin \theta \hat{\mathbf{e}}_{\theta} .
\end{gathered}
$$

É conveninete observar a seguinte relação entre os sistemas de coordenadas apresentados acima:

$$
\hat{\mathbf{e}}_{R}=\cos \theta \hat{\mathbf{e}}_{r}-\sin \theta \hat{\mathbf{e}}_{\theta}, \quad \hat{\mathbf{e}}_{\varphi}=-\hat{\mathbf{e}}_{\phi}, \quad \hat{\mathbf{e}}_{Z}=\sin \theta \hat{\mathbf{e}}_{r}+\cos \theta \hat{\mathbf{e}}_{\theta} .
$$




\section{Apêndice C}

\section{Obtenção das expressões analíticas referentes à análise de equilíbrio com}

\section{rotação}

O principal intuito deste apêndice mostrar a metodologia para obtenção das equações (3.17)(3.19), que descrevem o equilíbrio MHD com rotação poloidal e toroidal.

\section{C.1 Relações envolvendo B e J}

Inicialmente, a partir do campo magnético de equilíbrio [50],

$$
\mathbf{B}=F \nabla \phi+\nabla \phi \times \nabla \Psi
$$

a densidade de corrente a ele associado pode ser obtida pelo uso das identidades (B.7) e (B.9), resultando em

$$
\mathbf{J}=\mu_{0}^{-1} \nabla \times \mathbf{B}=\mu_{0}^{-1}[-\nabla \phi \times \nabla F+(\boldsymbol{\nabla} \cdot \nabla \Psi) \nabla \phi+(\nabla \Psi \cdot \nabla) \nabla \phi-(\nabla \phi \cdot \nabla) \nabla \Psi],
$$

Para o desenvolvimento algébrico de (C.2), podemos escrever os dois últimos termos do lado 
direito em coordenadas cilíndricas, de acordo com (B.13), considerando para isso $\phi=-\varphi$, ou seja, de forma explícita segue que

$$
\begin{aligned}
& (\boldsymbol{\nabla} \Psi \cdot \boldsymbol{\nabla}) \boldsymbol{\nabla} \phi-(\boldsymbol{\nabla} \phi \cdot \boldsymbol{\nabla}) \boldsymbol{\nabla} \Psi= \\
& \frac{\partial \Psi}{\partial R} \frac{\partial}{\partial R}\left(\frac{-\hat{\mathbf{e}}_{\varphi}}{R}\right)+\frac{1}{R^{2}} \frac{\partial}{\partial \varphi}\left(\frac{\partial \Psi}{\partial R} \hat{\mathbf{e}}_{R}\right)=\frac{2}{R^{2}} \frac{\partial \Psi}{\partial R} \hat{\mathbf{e}}_{\varphi}=R^{2}\left[\nabla\left(\frac{1}{R^{2}}\right) \cdot \nabla \Psi\right] \nabla \phi .
\end{aligned}
$$

Com o uso de (C.3) e de (B.6) torna-se conveniente expressar a densidade de corrente em termos do operador de Shafranov, $\Delta^{*} \Psi=\nabla \cdot\left(\nabla \Psi / R^{2}\right)$, de forma que

$$
\mathbf{J}=\mu_{0}^{-1}\left(R^{2} \Delta^{*} \Psi \nabla \phi-\nabla \phi \times \nabla F\right)
$$

A partir das expressões analítica para $\mathbf{B}$ e $\mathbf{J}$ apresentadas acima, obtém-se

$$
\begin{aligned}
& \boldsymbol{\nabla} \phi \times \mathbf{B}=-\frac{\nabla \Psi}{R^{2}}, \quad(\boldsymbol{\nabla} \phi \times \nabla \Psi) \times \mathbf{B}=\frac{F}{R^{2}} \nabla \Psi \\
& \nabla \Psi \times \mathbf{B}=|\nabla \Psi|^{2} \nabla \phi-F(\boldsymbol{\nabla} \phi \times \nabla \Psi)=B^{2} R^{2} \nabla \phi-F \mathbf{B}, \quad B^{2}=\frac{F^{2}+|\nabla \Psi|^{2}}{R^{2}},
\end{aligned}
$$

$$
\begin{aligned}
& \boldsymbol{\nabla} \phi \times(\boldsymbol{\nabla} \times \mathbf{B})=\frac{\boldsymbol{\nabla} F}{R^{2}}, \quad(\boldsymbol{\nabla} \phi \times \nabla \Psi) \times(\boldsymbol{\nabla} \times \mathbf{B})=-\left(\Delta^{*} \Psi\right) \nabla \Psi-(\mathbf{B} \cdot \boldsymbol{\nabla} F) \boldsymbol{\nabla} \phi, \\
& \nabla \Psi \times(\boldsymbol{\nabla} \times \mathbf{B})=-R^{2} \Delta^{*} \Psi(\boldsymbol{\nabla} \phi \times \nabla \Psi)-(\boldsymbol{\nabla} \Psi \cdot \nabla F) \boldsymbol{\nabla} \phi,
\end{aligned}
$$

onde foram utilizadas as identidades (B.2) e, com relação a simetria azimutal, $\boldsymbol{\nabla} \phi \cdot \boldsymbol{\nabla} f=0$ (para qualquer função $f$ ) em (C.5) e (C.6).

O termo devido a força magnética é calculado abaixo utilizando (C.6),

$$
\mathbf{J} \times \mathbf{B}=-\frac{1}{\mu_{0} R^{2}}\left[\left(\Delta^{*} \Psi\right) \nabla \Psi+\frac{1}{2} \nabla F^{2}-(\mathbf{B} \cdot \nabla F) R^{2} \nabla \phi\right],
$$

\section{C.2 Relações para V}

Propriedades importantes da velocidade de equilíbrio podem ser determinadas a partir da lei de Ohm e a equação da continuidade,

$$
\boldsymbol{E}+\mathbf{V} \times \mathbf{B}=0
$$




$$
\boldsymbol{\nabla} \cdot(\rho \mathbf{V})=0
$$

onde, na primeira, desconsideramos o efeito diamagnético, condizente com a ordem MHD.

De (C.8), segue que $\mathbf{V}=\mathbf{V}^{\prime}+C \mathbf{B}$, com $\mathbf{V}^{\prime} \perp \mathbf{B}$, é uma solução possível, onde $\mathbf{V}^{\prime}$ pode ser determinado a partir do produto de $\mathbf{B} / B^{2}$ com (C.8),

$$
\mathbf{V}^{\prime}=\frac{\boldsymbol{E} \times \mathbf{B}}{B^{2}}=\frac{F \boldsymbol{\nabla} \phi \times \boldsymbol{\nabla} \Phi-(\boldsymbol{\nabla} \Psi \cdot \nabla \Phi) \boldsymbol{\nabla} \phi}{B^{2}}
$$

Como $\boldsymbol{E} \cdot \mathbf{B}=-\boldsymbol{\nabla} \Phi \cdot(\boldsymbol{\nabla} \phi \times \nabla \Psi)=0$ e $\boldsymbol{\nabla} \Phi \cdot \nabla \Psi=0$, por simetria azimutal, conclui-se que $\boldsymbol{E}$ está na direção de $\boldsymbol{\nabla} \Psi$, ou, de forma equivalente,

$$
\boldsymbol{E}=-\Omega \nabla \Psi, \quad \Omega=\Omega(\Psi)=\frac{d \Phi}{d \Psi},
$$

o que permite desenvolver (C.10), de acordo com

$$
\mathbf{V}^{\prime}=-\Omega R^{2} \nabla \phi+\frac{F \Omega}{B^{2}} \mathbf{B}
$$

e, consequentemente, podemos expressar a velocidade $\mathbf{V}$ como

$$
\mathbf{V}=C^{\prime} \mathbf{B}-\Omega R^{2} \nabla \phi, \quad C^{\prime}=C+\frac{F \Omega}{B^{2}} .
$$

Esta, quando substituída em (C.9), com o auxílio de (B.6), $\boldsymbol{\nabla} \cdot \mathbf{B}=0$ e (B.16) leva à seguinte equação:

$$
\boldsymbol{\nabla} \cdot(\rho C \mathbf{B})-\boldsymbol{\nabla} \cdot\left(\rho \Omega R^{2} \boldsymbol{\nabla} \phi\right)=\mathbf{B} \cdot \boldsymbol{\nabla}(\rho C)=0,
$$

que pode ser traduzida para $C=\kappa(\Psi) / \rho$, pois $\mathbf{B} \cdot \nabla f=0$ implica que $f=f(\Psi)$, para qualquer função $f$ simétrica em $\phi$. Fica, portanto, determinada a velocidade de equilíbrio em função das grandezas $\kappa(\Psi), \Omega(\Psi), F$ e $\Psi$ :

$$
\mathbf{V}=\frac{\kappa}{\rho} \mathbf{B}-\Omega R^{2} \nabla \phi,
$$


onde $\kappa=\kappa(\Psi)$ é uma função arbitrária de fluxo diretamente relacionanda com a velocidade poloidal.

Da mesma forma como foram obtidos (C.5) e (C.6), é conveniente obter relações envolvendo o produto vetorial com (C.15):

$$
\begin{aligned}
& \nabla \phi \times \mathbf{V}=-\frac{\kappa}{\rho R^{2}} \nabla \Psi, \quad \mathbf{B} \times \mathbf{V}=-\Omega \nabla \Psi \\
& \nabla \Psi \times \mathbf{V}=\frac{\kappa|\nabla \Psi|^{2}}{\rho} \nabla \phi+\left(\Omega R^{2}-\frac{\kappa F}{\rho}\right)(\nabla \phi \times \nabla \Psi)
\end{aligned}
$$

A próxima etapa é o cálculo da força Centrífuga e de Coriolis devido a rotação do plasma na equação de momento. Para este cálculo, é conveniente utilizar a seguinte identidade

$$
\mathbf{V} \cdot \nabla \mathbf{V}=\boldsymbol{\nabla}\left(\frac{V^{2}}{2}\right)-\mathbf{V} \times(\boldsymbol{\nabla} \times \mathbf{V})
$$

obtida a partir de (B.10), que é mais geral. Para evitar expressões muito extensas, é conveniente calcular as componentes de (C.17) na direção de um vetor arbritário $\mathbf{U}$, de acordo com a identidade

$$
\mathbf{U V}: \nabla \mathbf{V}=\mathbf{U} \cdot(\mathbf{V} \cdot \nabla \mathbf{V})=\mathbf{U} \cdot \nabla\left(\frac{V^{2}}{2}\right)-(\mathbf{U} \times \mathbf{V}) \cdot(\boldsymbol{\nabla} \times \mathbf{V})
$$

proveniente do uso de (B.1). A este vetor arbritário, atribuiremos $\mathbf{U}=\boldsymbol{\nabla} \phi, \mathbf{U}=\mathbf{B}$ e $\mathbf{U}=\boldsymbol{\nabla} \Psi$, para obter as equações (3.17)-(3.19).

Para o desenvolvimento de (C.18) é necessário, primeiramente, efetuar os seguintes cálculos:

$$
\begin{gathered}
\frac{V^{2}}{2}=\frac{1}{2} \frac{\kappa^{2} B^{2}}{\rho^{2}}+\frac{1}{2} \Omega^{2} R^{2}-\frac{\kappa F \Omega}{\rho}, \\
\nabla \times \mathbf{V}=\left[\frac{\kappa}{\rho} \Delta^{*} \Psi+\nabla \Psi \cdot \nabla\left(\frac{\kappa}{\rho}\right)\right] \nabla \phi+\nabla \phi \times \nabla\left(\Omega R^{2}-\frac{\kappa F}{\rho}\right),
\end{gathered}
$$

onde o segundo, de considerável extensão algébria, foi feito a partir da relação (C.4) e das identidades (B.1) e (B.7). 
De (C.16), (C.18) e (C.20) obtemos as componentes da força inercial devido à rotação:

$$
\begin{gathered}
\rho \boldsymbol{\nabla} \phi \mathbf{V}: \nabla \mathbf{V}=\frac{1}{R^{2}} \mathbf{B} \cdot \nabla\left(\frac{\kappa^{2} F}{\rho}-\kappa \Omega R^{2}\right), \\
\rho \mathbf{B V}: \nabla \mathbf{V}=\rho \mathbf{B} \cdot \nabla\left(\frac{\kappa^{2} B^{2}}{2 \rho^{2}}-\frac{\Omega^{2} R^{2}}{2}\right),
\end{gathered}
$$

$$
\begin{aligned}
& \rho \nabla \Psi \mathbf{V}: \nabla \mathbf{V}=\frac{|\nabla \Psi|^{2}}{R^{2}}\left[-\frac{\kappa^{2}}{\rho} \Delta^{*} \Psi-\rho \nabla \Psi \cdot \nabla\left(\frac{\kappa^{2}}{2 \rho^{2}}\right)+\frac{\rho}{|\nabla \Psi|^{2}} \nabla \Psi \cdot \nabla\left(\frac{\kappa^{2}}{\rho^{2}}|\nabla \Psi|^{2}\right)\right]+ \\
& \left(\frac{\kappa F \Omega}{\rho}-\frac{\Omega R^{2}}{2}-\frac{\kappa^{2} B^{2}}{2 \rho^{2}}\right) \nabla \Psi \cdot \nabla R^{2},
\end{aligned}
$$

as quais, para obtenção das eqs. (3.17)-(3.19), devem ser somadas e subtraídas, respectivamente, às componentes do gradiente de pressão e da força magnética. É conveniente para este cálculo, portanto, utilizar os seguintes resultados:

$$
\begin{gathered}
\boldsymbol{\nabla} \phi \cdot(\mathbf{J} \times \mathbf{B})=\frac{\mathbf{B} \cdot \nabla F}{\mu_{0} R^{2}}, \\
\nabla \Psi \cdot(\mathbf{J} \times \mathbf{B})=-\frac{|\nabla \Psi|^{2}}{\mu_{0} R^{2}}\left(\Delta^{*} \Psi+\frac{1}{2} \frac{\nabla \Psi \cdot \nabla F^{2}}{|\nabla \Psi|^{2}}\right),
\end{gathered}
$$

obtidos a partir de (C.7). As referidas equações, após o desenvolvimento algébrico das componentes da equação de momento,

$$
\rho \mathbf{U V}: \nabla \mathbf{V}+\mathbf{U} \cdot \nabla p-\mathbf{U} \cdot(\mathbf{J} \times \mathbf{B})=0, \quad \mathbf{U}=\boldsymbol{\nabla} \phi, \mathbf{B}, \boldsymbol{\nabla} \Psi
$$

podem ser expressas na forma:

- Componente $\nabla \phi$ :

$$
\mathbf{B} \cdot \boldsymbol{\nabla}\left[F\left(1-\frac{\mu_{0} \kappa^{2}}{\rho}\right)+\mu_{0} \kappa \Omega R^{2}\right]=0
$$


- Componente B:

$$
\mathbf{B} \cdot \boldsymbol{\nabla}\left(\frac{\kappa^{2} B^{2}}{2 \rho^{2}}-\frac{\Omega^{2} R^{2}}{2}\right)+\frac{\mathbf{B} \cdot \nabla p}{\rho}=0
$$

- Componente $\nabla \Psi$ :

$$
\begin{aligned}
& \left(1-\frac{\mu_{0} \kappa^{2}}{\rho}\right) \Delta^{*} \Psi+\mu_{0} R^{2} \frac{\nabla \Psi \cdot \nabla p}{|\nabla \Psi|^{2}}+\frac{1}{2} \frac{\nabla \Psi \cdot \nabla F^{2}}{|\nabla \Psi|^{2}}-\frac{\mu_{0} \rho}{2} \nabla \Psi \cdot \nabla\left(\frac{\kappa^{2}}{\rho^{2}}\right)+ \\
& \frac{\mu_{0} \rho}{2|\nabla \Psi|^{2}} \nabla \Psi \cdot \nabla\left(\frac{\kappa^{2}}{\rho^{2}}|\nabla \Psi|^{2}\right)+\frac{\mu_{0} R^{2}}{|\nabla \Psi|^{2}}\left(\frac{\kappa F \Omega}{\rho}-\frac{\Omega R^{2}}{2}-\frac{\kappa^{2} B^{2}}{2 \rho^{2}}\right) \nabla \Psi \cdot \nabla R^{2} .
\end{aligned}
$$

\section{C.3 Cálculo de $\nabla \cdot \mathrm{q}$ de equilíbrio}

Para fechar o sistema, é necessário o cálculo de $\boldsymbol{\nabla} \cdot \mathbf{q}$, presente na eq. (3.15), que pode ser efetuado a partir da aplicação da identidade (B.6) na equação da definição de fluxo de calor, (3.11), o que resulta em

$$
\boldsymbol{\nabla} \cdot \mathbf{q}=\frac{\gamma}{\gamma-1}\left[\frac{p}{e B^{2}} \boldsymbol{\nabla} \cdot(\mathbf{B} \times \boldsymbol{\nabla} T)+(\mathbf{B} \times \boldsymbol{\nabla} T) \cdot \boldsymbol{\nabla}\left(\frac{p}{e B^{2}}\right)\right]
$$

No que se refere ao primeiro termo entre colchetes, o uso de (B.8) e (C.4) permite obter a relação aproximada,

$$
\boldsymbol{\nabla} \cdot(\mathbf{B} \times \nabla T)=(\nabla \phi \times \nabla T) \cdot \nabla F \approx \frac{d T_{0}}{d \Psi} \mathbf{B} \cdot \nabla F-\frac{d F_{0}}{d \Psi} \mathbf{B} \cdot \nabla T
$$

onde, além da aplicação de (B.1), a teoria de perturbação atemporal foi utilizada na última passagem, que emprega a aproximação: $\left|T_{1}(\Psi, \theta)\right| \ll T_{0}(\Psi)$ e $\left|F_{1}(\Psi, \theta)\right| \ll\left|F_{0}(\Psi)\right|$.

De forma similar, o segundo termo entre colchetes em (C.30) pode ser desenvolvido de acordo com a expressão

$$
\begin{aligned}
& (\mathbf{B} \times \nabla T) \cdot \nabla\left(\frac{p}{e B^{2}}\right) \approx \frac{F_{0}}{e}\left[\frac{d T_{0}}{d \Psi} \mathbf{B} \cdot \nabla\left(\frac{p}{B^{2}}\right)-\frac{d}{d \Psi}\left(\frac{p}{B^{2}}\right) \mathbf{B} \cdot \nabla T\right] \approx \\
& \frac{p_{0} R_{0}^{2}}{e F_{0}}\left\{\frac{d T_{0}}{d \Psi}\left[\frac{\mathbf{B} \cdot \nabla p}{p_{0}}-\frac{2 \mathbf{B} \cdot \boldsymbol{\nabla} F}{F_{0}}+\frac{\mathbf{B} \cdot \boldsymbol{\nabla} R^{2}}{R_{0}^{2}}\right]-\left[\frac{1}{p_{0}} \frac{d p_{0}}{d \Psi}-\frac{2}{F_{0}} \frac{d F_{0}}{d \Psi}+\frac{1}{R_{0}^{2}} \frac{d R^{2}}{d \Psi}\right] \mathbf{B} \cdot \nabla T\right\}
\end{aligned}
$$

onde foi considerado que $|\nabla \Psi| \ll|F|,|\mathbf{B} \cdot \nabla(|\nabla \Psi|)| \ll|\mathbf{B} \cdot \nabla F|$ e $d|\nabla \Psi| / d \Psi \ll|d F / d \Psi|$. 
Com as definições introduzidas pelas eqs. (3.20), (3.23), (3.24) e (3.26), as equações (C.31) e (C.32) podem ser expressas na forma

$$
\begin{aligned}
& \frac{p}{e B^{2}} \nabla \cdot(\mathbf{B} \times \nabla T)=M_{\mathrm{th}}\left(\Delta_{F}-\mathcal{R}_{F} \Delta_{T}\right) \frac{p_{0} c_{s}}{F_{0}} \frac{\mathbf{B} \cdot \nabla R^{2}}{R_{0}}, \quad(\mathbf{B} \times \nabla T) \cdot \nabla\left(\frac{p}{e B^{2}}\right)= \\
& M_{\mathrm{th}}\left[1-2 \Delta_{F}+\Delta_{p}-\left(1+\mathcal{R}_{\rho}-2 \mathcal{R}_{F}+\frac{T_{0}}{R_{0}^{2}} \frac{\partial R^{2} / \partial \Psi}{d T_{0} / d \Psi}\right) \Delta_{T}\right] \frac{p_{0} c_{s} \mathbf{B} \cdot \boldsymbol{\nabla} R^{2}}{F_{0}}
\end{aligned}
$$

Em resumo, ao assumir que as grandezas de equilíbrio são da forma: $X=X_{0}(\Psi)+Z X_{1}(\Psi, \theta)$ com $\left|X_{1}\right| \ll\left|X_{0}\right|$ e ao desprezarmos o termo $|\nabla \Psi|^{2} / F_{0}^{2}$ e suas derivadas e gradientes, pudemos desenvolver a expressão da divergência do fluxo de calor e obter o seguinte resultado:

$$
\boldsymbol{\nabla} \cdot \mathbf{q}=M_{\mathrm{th}}\left[1-\Delta_{F}+\Delta_{p}-\left(1+\mathcal{R}_{\rho}-\mathcal{R}_{F}+\frac{T_{0}}{R_{0}^{2}} \frac{\partial R^{2} / \partial \Psi}{d T_{0} / d \Psi}\right) \Delta_{T}\right] \frac{\gamma p_{0} c_{s} R_{0}}{(\gamma-1) F_{0}} \frac{\mathbf{B} \cdot \nabla R^{2}}{R_{0}^{2}}
$$

o qual é utilizado em 3.2. 


\section{Apêndice D}

\section{Derivação de fórmulas usadas no capítulo 3}

Expressões algébricas para o divergente da velocidade, do tensor de viscosidade paralela e da densidade de corrente e a equação de evolução do tensor de viscosidade paralela, ambas bastante utilizadas no capítulo 3 , são obtidas neste apêndice. O índice $\alpha=i, e$ das quantidades macroscópicas do plasma é suprimido nas expressões para simplificar a notação, contudo, eles devem ficar subentendidos. Os resultados aqui apresentados são válidos, em sua maiorida, para sistemas de baixa pressão $\left(\beta \sim \varepsilon^{2}\right)$.

\section{D.1 Relações para B}

Para a obtenção de futuras relações, é conveniente separar as componentes paralela e perpendicular (com relação a $\mathbf{b}=\mathbf{B} / B$ ) do operador $\boldsymbol{\nabla}$, ou seja,

$$
\nabla_{\|}=\mathbf{b} \cdot \nabla \quad \text { e } \quad \nabla_{\perp}=\nabla-\mathbf{b} \nabla_{\|}
$$

de forma que, a partir de (B.6) e (B.7) obtém-se

$$
\boldsymbol{\nabla} \cdot \mathbf{b}=\nabla_{\|} \ln B, \quad \boldsymbol{\nabla} \times \mathbf{b}=\mu_{0} \frac{\mathbf{J}}{B}+\mathbf{b} \times \nabla \ln B \approx \mathbf{b} \times \nabla \ln B
$$


se considerarmos $J_{\|} \sim J_{\perp}$, pois $\nabla p \approx \mathbf{J} \times \mathbf{B}$ e, consequentemente,

$$
\frac{\left|\mu_{0} \mathbf{J}_{\perp} / B\right|}{|\boldsymbol{\nabla} \times \mathbf{b}|} \sim \beta, \quad \mathbf{b} \cdot(\boldsymbol{\nabla} \times \mathbf{b})=\mu_{0} \frac{J_{\|}}{B} \sim \frac{\beta}{L} .
$$

Utilizando (B.2) e (D.2), obtém-se a seguinte aproximação

$$
\boldsymbol{\kappa}=\nabla_{\|} \mathbf{b}=-\mathbf{b} \times(\boldsymbol{\nabla} \times \mathbf{b}) \approx \nabla_{\perp} \ln B
$$

para o vetor de curvatura do campo magnético $(\boldsymbol{\kappa})$.

Para os cálculos da próxima seção é conveniente definir $\mathbf{G}=f \mathbf{B} \times \nabla g^{1}$, cujo cálculo do divergente, efetuado a partir de (B.6) e (B.8), resulta em

$$
\nabla \cdot \mathbf{G} \approx \mathbf{G} \cdot \nabla \ln f
$$

Em particular, para $f=B^{-1}, \boldsymbol{\nabla} \cdot \mathbf{G}=(\mathbf{b} \times \boldsymbol{\kappa}) \cdot \boldsymbol{\nabla} g$ e, neste caso, obtém-se ${ }^{2}$ :

$$
\mathbf{v}_{D}=\frac{\mathbf{b} \times \boldsymbol{\nabla} g}{B}, \quad \boldsymbol{\nabla} \cdot \mathbf{v}_{D}=-2 \mathbf{v}_{D} \cdot \nabla \ln B
$$

\section{D.2 Cálculo da divergência de $\pi, \mathrm{v}, \mathrm{J}$ e q}

Iniciamos esta seção apresentando a definição de viscosidade paralela:

$$
\boldsymbol{\pi}_{\|}=\frac{3}{2} \pi_{\|}\left(\mathbf{b b}-\frac{1}{3} \mathbf{I}\right)
$$

cujo cálculo de seu divergente, obtido a partir de (B.12) e (B.6) e das relações apresentadas na seção anterior, resulta em

$$
\boldsymbol{\nabla} \cdot \boldsymbol{\pi}_{\|}=\frac{3}{2}\left[\left(\mathbf{b} \nabla_{\|} \ln B+\boldsymbol{\kappa}\right) \pi_{\|}+\mathbf{b} \nabla_{\|} \pi_{\|}\right]-\frac{1}{2} \boldsymbol{\nabla} \pi_{\|} .
$$

\footnotetext{
${ }^{1}$ Aqui, G é um vetor arbitrário, mais adiante utilizado para representar $\mathbf{v}, \mathbf{J}$, etc.

${ }^{2}$ Note que $\mathbf{v}_{D}$ possui a forma de uma velocidade de deriva.
} 
Note também que

$$
\begin{gathered}
\mathbf{b} \cdot \boldsymbol{\nabla} \cdot \boldsymbol{\pi}_{\|}=\frac{3}{2} \pi_{\|} \nabla_{\|} \ln B+\nabla_{\|} \pi_{\|}, \\
\mathbf{b} \times \nabla \cdot \boldsymbol{\pi}_{\|}=\frac{3}{2} \pi_{\|}(\mathbf{b} \times \boldsymbol{\kappa})-\frac{1}{2} \mathbf{b} \times \nabla_{\perp} \pi_{\|},
\end{gathered}
$$

e, utilizando (B.8),

$$
\boldsymbol{\nabla} \cdot\left(\mathbf{b} \times \nabla \cdot \boldsymbol{\pi}_{\|}\right)=(\mathbf{b} \times \boldsymbol{\kappa}) \cdot \nabla \pi_{\|} \cdot
$$

\section{D.2.1 Relações para velocidades (v)}

Utilizando (D.6) e (B.6), obtemos um primeiro desenvolvimento algébrico para o divergente das principais velocidades de deriva em fluidos. Neste desenvolvimento consideramos a ordem $\operatorname{MHD}\left(v_{E} \sim v_{T_{i}}\right)$ e não levamos em conta o termo de giroviscosidade $\left(\boldsymbol{\pi}_{g}\right)$ e viscosidade perpendicular $\left(\boldsymbol{\pi}_{\perp}\right)$, os quais são de ordem superior em $\rho_{i} / L$. A seguir apresentamos tais resultados:

$$
\begin{gathered}
\mathbf{v}_{E}=\frac{\mathbf{b} \times \boldsymbol{\nabla} \Phi}{B}, \quad \mathbf{v}_{p}=\frac{\mathbf{b} \times \boldsymbol{\nabla} p}{e n B}, \quad \mathbf{v}_{\pi}=\frac{\mathbf{b} \times \boldsymbol{\nabla} \cdot \boldsymbol{\pi}}{e n B}, \quad \mathbf{v}_{I} \approx \frac{\mathbf{b}}{\omega_{c}} \times \frac{d \mathbf{v}_{E}}{d t}, \\
\boldsymbol{\nabla} \cdot\left(n \tilde{\mathbf{v}}_{E}\right)=\tilde{\mathbf{v}}_{E} \cdot \nabla n-2 n \tilde{\mathbf{v}}_{E} \cdot \boldsymbol{\nabla} \ln B, \\
\boldsymbol{\nabla} \cdot\left(n \mathbf{v}_{p}\right)=-2 n \mathbf{v}_{p} \cdot \boldsymbol{\nabla} \ln B, \\
\boldsymbol{\nabla} \cdot\left(n \mathbf{v}_{\pi}\right)=n \mathbf{v}_{\pi} \cdot \boldsymbol{\nabla} \ln B, \\
\boldsymbol{\nabla} \cdot\left(n \mathbf{v}_{I}\right)=\mathbf{v}_{I} \cdot \boldsymbol{\nabla} n-n \mathbf{v}_{I} \cdot \boldsymbol{\nabla} \ln B+\frac{1}{\omega_{c}} \boldsymbol{\nabla} \cdot\left(\mathbf{b} \times \frac{d \mathbf{v}_{E}}{d t}\right)
\end{gathered}
$$




$$
\boldsymbol{\nabla} \cdot\left(v_{\|} \mathbf{b}\right)=\nabla_{\|} v_{\|}-v_{\|} \nabla_{\|} \ln B
$$

\section{D.2.2 Relações para a densidade de corrente (j)}

De forma similar à subseção anterior, obtemos as expressões das principais componentes da densidade de corrente e seus respectivos divergentes:

$$
\begin{gathered}
\mathbf{j}_{p}=\frac{\mathbf{b} \times \boldsymbol{\nabla} p}{B}, \quad \mathbf{j}_{\pi}=\frac{\mathbf{b} \times \boldsymbol{\nabla} \cdot \boldsymbol{\pi}_{\|}}{B}, \quad \mathbf{j}_{\mathrm{I}} \approx \frac{m n}{B} \mathbf{b} \times \frac{d \mathbf{v}_{E}}{d t}, \\
\boldsymbol{\nabla} \cdot \mathbf{j}_{p}=-2 \mathbf{j}_{p} \cdot \boldsymbol{\nabla} \ln B, \\
\boldsymbol{\nabla} \cdot \mathbf{j}_{\pi}=\mathbf{j}_{\pi} \cdot \boldsymbol{\nabla} \ln B, \\
\nabla \cdot \mathbf{j}_{\mathrm{I}} \approx \mathbf{j}_{\mathrm{I}} \cdot \nabla \ln \left(\frac{n}{B}\right)-\frac{m n}{B}\left[\frac{\partial}{\partial t} \boldsymbol{\nabla} \cdot\left(\frac{\boldsymbol{\nabla}_{\perp} \Phi}{B}\right)+\boldsymbol{\nabla} \cdot\left(\mathbf{b} \times \mathbf{v}_{E} \cdot \nabla \mathbf{v}_{E}\right)\right] .
\end{gathered}
$$

\section{D.3 Equação de evolução de $\pi_{\|}$}

Primeiramente observamos que o termo $T_{i j k}=\hat{\mathbf{e}}_{i} \cdot\left[\left(\hat{\mathbf{e}}_{j} \cdot \nabla\right) \hat{\mathbf{e}}_{k}\right], i, j, k=0,1,2$, satisfaz genericamente as seguintes relações:

$$
\begin{gathered}
T_{i j k}=-T_{i k j}+\hat{\mathbf{e}}_{i} \cdot\left[\left(\boldsymbol{\nabla} \times \hat{\mathbf{e}}_{j}\right) \times \hat{\mathbf{e}}_{k}\right]+\hat{\mathbf{e}}_{i} \cdot\left[\left(\boldsymbol{\nabla} \times \hat{\mathbf{e}}_{k}\right) \times \hat{\mathbf{e}}_{j}\right], \\
T_{00 k}=-T_{0 k 0}-T_{k 00}, \quad \hat{\mathbf{e}}_{0}=\mathbf{b} .
\end{gathered}
$$

Como $\mathbf{b} \cdot d \mathbf{b} / d t=0, \mathbf{b} \cdot \partial \mathbf{b} / \partial t=0$ e $\mathbf{b} \cdot \boldsymbol{\kappa}=0$, seque que

$$
\mathbf{b} \cdot \frac{d \mathbf{b}}{d t}=\mathbf{b} \cdot\left(\frac{\partial \mathbf{b}}{\partial t}+\left(\mathbf{v}_{\perp} \cdot \nabla\right) \mathbf{b}+v_{\|} \boldsymbol{\kappa}\right)=\mathbf{b v}_{\perp}: \nabla \mathbf{b}=0
$$


e, consequentemente, como $\mathbf{v}_{\perp}=v_{\perp 1} \hat{\mathbf{e}}_{1}+v_{\perp 2} \hat{\mathbf{e}}_{2}$,

$$
v_{\perp 1} T_{010}+v_{\perp 2} T_{020}=0
$$

Desta forma, de acordo com (D.4), (D.23) e (D.25), ao notar que, na forma matricial, as componentes de $\boldsymbol{\nabla v}$ e o termo bb $: \nabla \mathbf{v}$ podem ser expressos respectivamente como:

$$
M_{i j}=\left(\hat{\mathbf{e}}_{i} \cdot \nabla\right) v_{j}+\sum_{m=0}^{2} v_{m} T_{i j m}, \quad \sum_{i, j} b_{i} M_{i j} b_{j}=b_{0} M_{00} b_{0}=M_{00}
$$

onde $v_{0}=v_{\|}, v_{1}=v_{\perp 1}$ e $v_{2}=v_{\perp 2}$, obtém-se a relação:

$$
\mathbf{b b}:(\nabla \mathbf{v})^{T}=\mathbf{b b}: \nabla \mathbf{v}=\nabla_{\|} v_{\|}-\mathbf{v}_{\perp} \cdot \nabla \ln B .
$$

Consideramos agora a equação de evolução do tensor viscosidade [37,73],

$$
\begin{aligned}
& \frac{d \boldsymbol{\pi}}{d t}+\boldsymbol{\pi} \boldsymbol{\nabla} \cdot \mathbf{v}+\left[\boldsymbol{\pi} \cdot \boldsymbol{\nabla} \mathbf{v}+(\boldsymbol{\pi} \cdot \boldsymbol{\nabla} \mathbf{v})^{T}-(\gamma-1) \mathbf{I}(\boldsymbol{\pi}: \boldsymbol{\nabla} \mathbf{v})\right]+\omega_{c}(\mathbf{b} \times \boldsymbol{\pi}-\boldsymbol{\pi} \times \mathbf{b})+ \\
& {\left[p \boldsymbol{\nabla} \mathbf{v}+p(\boldsymbol{\nabla} \mathbf{v})^{T}-(\gamma-1) p \boldsymbol{\nabla} \cdot \mathbf{v}\right]+\frac{\gamma-1}{\gamma}\left[\boldsymbol{\nabla} \mathbf{q}+(\boldsymbol{\nabla} \mathbf{q})^{T}-(\gamma-1) \mathbf{I} \boldsymbol{\nabla} \cdot \mathbf{q}\right]+\boldsymbol{\nabla} \cdot \boldsymbol{\tau}=0}
\end{aligned}
$$

onde $\tau$ é um dos próximos momentos da função distribuição, que é considerado nulo neste contexto, e consideramos o caso não colisional.

Considerando também apenas o efeito de viscosidade paralela, ou seja, $\boldsymbol{\pi} \approx \boldsymbol{\pi}_{\|}=\pi_{\|}(\mathbf{b b}-\mathbf{I} / 3)$, de acordo com (D.24), obtém-se:

$$
\mathbf{b b}: \frac{d \boldsymbol{\pi}}{d t}=\frac{d \pi_{\|}}{d t}-\frac{d \mathbf{b}}{d t} \cdot(\mathbf{b} \cdot \boldsymbol{\pi})-\mathbf{b} \cdot\left(\frac{d \mathbf{b}}{d t} \cdot \boldsymbol{\pi}\right)=\frac{d \pi_{\|}}{d t}
$$

Desta forma, no regime linear, para o caso adiabático $(\mathbf{q}=0)$ em que não há rotação de equilíbrio, a partir de (D.27), (D.28) e (D.29), segue que

$$
\frac{d \pi_{\|}}{d t}+p\left[2 \nabla_{\|} v_{\|}-2 \mathbf{v}_{\perp} \cdot \nabla \ln B-(\gamma-1) \nabla \cdot \mathbf{v}\right]=0 .
$$




\section{D.4 Aproximação para tokamaks de superfícies mag- néticas concêntricas}

\section{D.4.1 Campo magnético de equilíbrio}

Em tokamaks de superfíces magnéticas aproximadamente concêntricas, conforme descrito no capítulo 2, o campo magnético de equilíbrio pode ser aproximado por

$$
\mathbf{B}=B \mathbf{b}, \quad B \approx B_{0}(1-\varepsilon \cos \theta), \quad \mathbf{b}=\frac{\varepsilon}{q} \hat{\mathbf{e}}_{\theta}+\hat{\mathbf{e}}_{\phi} \approx \hat{\mathbf{e}}_{\phi}, \quad \varepsilon \ll 1
$$

e, convenientemente, representado no sistema de coordenadas quasi-toroidais $(r, \theta, \phi)$. Consequentemente, considerando apenas termos dominantes, o desenvolvimento algébrico dos operadores $\nabla_{\|}$e $\nabla_{\perp}$ e do vetor de curvatura $(\boldsymbol{\kappa})$ resulta em:

$$
\nabla_{\|}=k_{\|}\left(\frac{\partial}{\partial \theta}+q \frac{\partial}{\partial \phi}\right), \quad \nabla_{\perp}=\hat{\mathbf{e}}_{r} \frac{\partial}{\partial r}+\hat{\mathbf{e}}_{\theta} k_{\theta} \frac{\partial}{\partial \theta}, \quad \boldsymbol{\kappa}=-\frac{\hat{\mathbf{e}}_{R}}{R_{0}}
$$

onde $k_{\|}=1 / q R_{0}, k_{\theta}=1 / r$ e, de acordo com (B.26), $\hat{\mathbf{e}}_{R}=\cos \theta \hat{\mathbf{e}}_{r}-\sin \theta \hat{\mathbf{e}}_{\theta}$. Também observa-se que, para $q \gg 1$,

$$
\boldsymbol{\nabla} \times \mathbf{b} \approx \mathbf{b} \times \boldsymbol{\kappa}=-\frac{\hat{\mathbf{e}}_{Z}}{R_{0}}, \quad \nabla \cdot \mathbf{b} \sim \mathbf{b} \cdot(\boldsymbol{\nabla} \times \mathbf{b})=\mathcal{O}\left(\varepsilon k_{\|}\right) \sim 0
$$

onde $\hat{\mathbf{e}}_{Z}=\sin \theta \hat{\mathbf{e}}_{r}+\cos \theta \hat{\mathbf{e}}_{\theta}$.

\section{D.4.2 Campo magnético perturbado}

Derivamos a seguir algumas relações para o campo magnético perturbado considerando que $\tilde{\mathbf{B}}=\tilde{\mathbf{B}}_{\perp}=\boldsymbol{\nabla} \times\left(\tilde{A}_{\|} \mathbf{b}\right)$, onde $\tilde{\mathbf{A}}$ é o potencial vetor. Segue, portanto, que

$$
\tilde{\mathbf{B}}=-\mathbf{b} \times \nabla \tilde{A}_{\|}+\tilde{A}_{\|}(\mathbf{b} \times \boldsymbol{\kappa}) \approx-\frac{1}{r} \frac{\partial \tilde{A}_{\|}}{\partial \theta} \hat{\mathbf{e}}_{r}+i \mathrm{k}_{r} \tilde{A}_{\|} \hat{\mathbf{e}}_{\theta}
$$


A partir de (D.34) obtém-se

$$
\begin{gathered}
\tilde{\nabla}_{\|}=\frac{\tilde{\mathbf{B}}}{B} \cdot \boldsymbol{\nabla} \approx \frac{1}{r B}\left(i \mathrm{k}_{r} \tilde{A}_{\|} \frac{\partial}{\partial \theta}-\frac{\partial \tilde{A}_{\|}}{\partial \theta} \frac{\partial}{\partial r}\right), \\
\nabla \times \tilde{\mathbf{B}} \approx i \mathrm{k}_{r} k_{\|} \frac{\partial \tilde{A}_{\|}}{\partial \theta} \hat{\mathbf{e}}_{r}+\mathrm{k}_{r}^{2} \tilde{A}_{\|} \mathbf{b} .
\end{gathered}
$$

\section{D.4.3 Velocidade e densidade de corrente.}

A partir das relações

$$
v_{T_{i}}^{2}=\frac{2 T_{i}}{m_{i}}, \quad \rho_{i}=\frac{v_{T_{i}}}{\omega_{c_{i}}}, \quad \frac{1}{B}=\frac{v_{T_{i}} \rho_{i}}{2} \frac{e}{T_{i}}, \quad \rho_{i} / r \ll \mathrm{k}_{r} \rho_{i} \ll 1
$$

considerando o potêncial eletrostático perturbado $(\tilde{\Phi})$, bem como a pressão $(\tilde{p})$ e a viscosidade paralela $\left(\tilde{\pi}_{\|}\right)$, utilizamos (D.12) e (D.13) para obter:

$$
\begin{gathered}
\tilde{\mathbf{v}}_{E}=\tilde{\omega}_{E} R_{0}\left(\hat{\mathbf{e}}_{\theta}+i \frac{\rho_{i} / r}{\mathrm{k}_{r} \rho_{i}} \frac{\partial \ln \tilde{\Phi}}{\partial \theta} \hat{\mathbf{e}}_{r}\right), \quad \tilde{\omega}_{E}=\frac{i \mathrm{k}_{r} \tilde{\Phi}}{B}=\frac{i \mathrm{k}_{r} \rho_{i}}{2} \frac{e \tilde{\Phi}}{T_{i}} \frac{v_{T_{i}}}{R_{0}} \\
\tilde{\mathbf{j}}_{p}=\frac{i}{2} \frac{e}{T_{i}} \omega_{\mathrm{di}} R_{0}\left(\tilde{p} \hat{\mathbf{e}}_{\theta}+i \frac{\rho_{i} / r}{\mathrm{k}_{r} \rho_{i}} \frac{\partial \tilde{p}_{i}}{\partial \theta} \hat{\mathbf{e}}_{r}\right), \quad \omega_{\mathrm{di}}=\mathrm{k}_{r} \rho_{i} \frac{v_{T_{i}}}{R_{0}} \\
\tilde{\mathbf{j}}_{\pi}=-\frac{i}{4} \frac{e}{T_{i}} \omega_{\mathrm{di}} R_{0}\left(\tilde{\pi}_{\| i} \hat{\mathbf{e}}_{\theta}+i \frac{\rho_{i} / r}{\mathrm{k}_{r} \rho_{i}} \frac{\partial \tilde{\pi}_{\| i}}{\partial \theta} \hat{\mathbf{e}}_{r}\right) \\
\tilde{\mathbf{j}}_{I}=i e n \tilde{\omega}_{E} R_{0} \frac{\omega}{\omega_{c}} \hat{\mathbf{e}}_{r} .
\end{gathered}
$$




\section{Apêndice E}

\section{Solução iterativa das equações}

\section{perturbadas da MHD ideal}

\section{E.1 Equações iniciais e solução de equilíbrio}

Neste apêndice apresentamos um método iterativo para resolver o sistema composto pelas eqs. (3.51) - (3.53), as quais são repetidas a seguir para facilitar a leitura:

$$
\begin{gathered}
\rho_{0} \frac{\partial \tilde{v}_{\|}}{\partial t}+\nabla_{\|} \tilde{p}+\tilde{F}_{\|}=0, \\
\frac{\partial(\tilde{\rho}+\tilde{R})}{\partial t}+\rho_{0} \boldsymbol{\nabla} \cdot \tilde{\mathbf{v}}=0, \\
\frac{\partial(\tilde{p}+\tilde{P})}{\partial t}+\gamma p_{0} \boldsymbol{\nabla} \cdot \tilde{\mathbf{v}}=0, \\
\tilde{\mathbf{v}}=\tilde{\mathbf{v}}_{E}+v_{\|} \mathbf{b}, \quad \tilde{\mathbf{v}}_{E} \approx \tilde{\omega}_{E} R_{0}(1+\varepsilon \cos \theta) \hat{\mathbf{e}}_{\theta}, \\
\tilde{F}_{\|}=\rho_{0}(\mathbf{b} \tilde{\mathbf{v}}: \boldsymbol{\nabla} \mathbf{V}+\mathbf{b} \mathbf{V}: \nabla \tilde{\mathbf{v}})+\tilde{\rho} \mathbf{b} \mathbf{V}: \nabla \mathbf{V},
\end{gathered}
$$




$$
\begin{gathered}
\tilde{R}=\int(\mathbf{V} \cdot \boldsymbol{\nabla} \tilde{\rho}+\tilde{\mathbf{v}} \cdot \boldsymbol{\nabla} \rho+\tilde{\rho} \boldsymbol{\nabla} \cdot \mathbf{V}) d t \\
\tilde{P}=\int\left(\mathbf{V} \cdot \boldsymbol{\nabla} \tilde{p}+\tilde{\mathbf{v}} \cdot \boldsymbol{\nabla} p_{0}+\gamma \tilde{p} \boldsymbol{\nabla} \cdot \mathbf{V}+(\gamma-1) \boldsymbol{\nabla} \cdot \tilde{\mathbf{q}}\right) d t .
\end{gathered}
$$

Para se obter a relação de dispersão, é necessário calcular a componente poloidal da força $\tilde{\mathbf{F}}$, ou seja,

$$
\tilde{F}_{\theta}=\rho_{0}\left(\hat{\mathbf{e}}_{\theta} \tilde{\mathbf{V}}: \nabla \mathbf{V}+\hat{\mathbf{e}}_{\theta} \mathbf{V}: \nabla \tilde{\mathbf{V}}\right)+\tilde{\rho} \hat{\mathbf{e}}_{\theta} \mathbf{V}: \nabla \mathbf{V}
$$

As expressões algébricas para as grandezas definidas por (E.5)-(E.8) são obtidas a partir do equilíbrio, que é descrito por:

$$
\begin{gathered}
\rho=\rho_{0}\left(1+2 \varepsilon \Delta_{\rho} \cos \theta\right), \\
p=p_{0}\left(1+2 \varepsilon \Delta_{p} \cos \theta\right), \\
\mathbf{V}=V_{P} \hat{\mathbf{e}}_{\theta}+V_{T} \hat{\mathbf{e}}_{\phi}, \\
V_{P} \approx \frac{\varepsilon}{q} M_{P} c_{s}, \quad V_{T}=\left(M_{T}+\Delta_{V} \varepsilon \cos \theta\right) c_{s}, \quad \Delta_{V}=M_{T}-2\left(1+\Delta_{\rho}\right) M_{P} .
\end{gathered}
$$

O método iterativo que utilizamos para resolver as eqs. (E.1)-(E.3) nas variáveis $\tilde{v}_{\|}, \tilde{\rho}$ e $\tilde{p}$ e para a obtenção da relação de dispersão é justificado por se tratar de um modelo linear, no qual o princípio da superposição se aplica. Tal método consiste em decompor as quantidades perturbadas na forma:

$$
\tilde{X}=\tilde{X}^{(0)}+\tilde{X}^{(\mathrm{T})}+\tilde{X}^{(\mathrm{P})}
$$

onde "0", "T" e "P" indicam as contribuições para as quantidades perturbadas devido a dinâmica sem rotação, com rotação exclusivamente toroidal e com rotação poloidal e toroidal, respecti- 
vamente. O problema inicial é, então, dividido em três partes: Primeiramente, sem incluir rotação de equilíbrio, encontra-se a solução mais simples. Posteriormente, a partir desta solução, determina-se a solução proveniente da rotação toroidal e, finalmente, ao incluir rotação poloidal, encontra-se a solução completa.

\section{E.2 Cálculo de $\tilde{F}_{\|}, \tilde{R}$ e $\tilde{P}$.}

\section{E.2.1 Termos de convecção e derivadas angulares}

O cálculo dos termos convectivos, efetuados a partir das eqs. (E.4), (E.11) e (B.17), resulta nas seguintes relações:

$$
\begin{aligned}
& \mathbf{V}_{P} \cdot \boldsymbol{\nabla}=M_{P} k_{\|} c_{s} \frac{\partial}{\partial \theta}, \quad \tilde{\mathbf{v}}_{E} \cdot \boldsymbol{\nabla}=\frac{\tilde{\omega}_{E}}{\varepsilon} \frac{\partial}{\partial \theta} \\
& \mathbf{V}_{T} \cdot \boldsymbol{\nabla}=q M_{T} k_{\|} c_{s} \frac{\partial}{\partial \phi}, \quad \tilde{v}_{\|} \mathbf{b} \cdot \boldsymbol{\nabla}=\frac{\tilde{v}_{\|}}{R_{0}} \frac{\partial}{\partial \phi} .
\end{aligned}
$$

Além dos termos convectivos, também é necessário, para o restante dos cálculos desta seção, obter as derivadas das velocidades com relação aos ângulos poloidal e toroidal, que, de acordo com (B.23)-(B.25) são:

$$
\begin{gathered}
\frac{\partial \mathbf{V}_{P}}{\partial \theta}=-\frac{\varepsilon}{q} M_{P} c_{s} \hat{\mathbf{e}}_{r}, \quad \frac{\partial \mathbf{V}_{T}}{\partial \theta}=-\varepsilon \Delta_{V} c_{s} \sin \theta \hat{\mathbf{e}}_{\phi}, \\
\frac{\partial \mathbf{V}_{P}}{\partial \phi} \approx 0, \quad \frac{\partial \mathbf{V}_{T}}{\partial \phi} \approx M_{T} c_{s}\left(-\cos \theta \hat{\mathbf{e}}_{r}+\sin \theta \hat{\mathbf{e}}_{\theta}\right) \\
\frac{\partial \tilde{\mathbf{v}}_{E}}{\partial \theta}=-\tilde{\omega}_{E} R_{0} \hat{\mathbf{e}}_{r}, \quad \frac{\partial\left(\tilde{v}_{\|} \mathbf{b}\right)}{\partial \theta}=\frac{\partial \tilde{v}_{\|}}{\partial \theta} \hat{\mathbf{e}}_{\phi} \\
\frac{\partial \tilde{\mathbf{v}}_{E}}{\partial \phi}=-\tilde{\omega}_{E} R_{0} \sin \theta \hat{\mathbf{e}}_{\phi}, \quad \frac{\partial\left(\tilde{v}_{\|} \mathbf{b}\right)}{\partial \phi}=\tilde{v}_{\|}\left(-\cos \theta \hat{\mathbf{e}}_{r}+\sin \theta \hat{\mathbf{e}}_{\theta}\right) .
\end{gathered}
$$




\section{E.2.2 Cálculo de $\tilde{F}_{\|}$e $\tilde{F}_{\theta}$}

Com a substituição de $\mathbf{V}=0, \mathbf{V}=\mathbf{V}_{T}$ e de $\mathbf{V}=\mathbf{V}_{P}$ em (E.5) obtém-se:

$$
\begin{gathered}
\tilde{F}_{\|}^{(0)}=0 \\
\tilde{F}_{\|}^{(\mathrm{T})}=\rho_{0}\left(\mathbf{b} \mathbf{V}_{T}: \nabla \tilde{\mathbf{v}}_{E}+\mathbf{b} \tilde{\mathbf{v}}_{E}: \nabla \mathbf{V}_{T}\right)=-\rho_{0} \tilde{\omega}_{E} c_{s}\left(M_{T}+\Delta_{V}\right) \sin \theta \\
\tilde{F}_{\|}^{(\mathrm{P})} \approx \rho_{0} \mathbf{b} \mathbf{V}_{P}: \nabla\left(\tilde{v}_{\|} \mathbf{b}\right)=M_{P} \rho_{0} k_{\|} c_{s} \frac{\partial \tilde{v}_{\|}}{\partial \theta}
\end{gathered}
$$

e, de forma similar, com relação a $\tilde{F}_{\theta}$, segue que

$$
\begin{gathered}
\tilde{F}_{\theta}^{(0)}=0, \quad \tilde{F}_{\theta}^{(\mathrm{T})}=\rho_{0}\left[\hat{\mathbf{e}}_{\theta} \mathbf{V}_{T}: \nabla\left(\tilde{v}_{\|} \mathbf{b}\right)+\tilde{v}_{\|} \hat{\mathbf{e}}_{\theta} \mathbf{b}: \nabla \mathbf{V}_{T}\right]+\left(\hat{\mathbf{e}}_{\theta} \mathbf{V}_{T}: \nabla \mathbf{V}_{T}\right) \tilde{\rho}= \\
q M_{T} k_{\|} c_{s}\left(2 \rho_{0} \tilde{v}_{\|}+M_{T} c_{s} \tilde{\rho}\right) \sin \theta, \quad \tilde{F}_{\theta}^{(\mathrm{P})}=0 .
\end{gathered}
$$

\section{E.2.3 Cálculo de $\tilde{R}$}

De acordo com (C.15), podemos desprezar o termo

$$
\boldsymbol{\nabla} \cdot \mathbf{V}=\mathbf{B} \cdot \boldsymbol{\nabla}\left(\frac{\kappa}{\rho}\right) \approx M_{P} k_{\|} c_{s} \rho_{0} \frac{\partial \rho^{-1}}{\partial \theta} \approx-2 \varepsilon M_{P} \Delta_{\rho} k_{\|} c_{s} \sin \theta=\mathcal{O}\left(\varepsilon M_{P} \Delta_{\rho} \varepsilon\right)
$$

que não contribui em primeira ordem para o cálculo de $\tilde{R}$, o qual é de (E.6) e resulta em:

$$
\begin{gathered}
\tilde{R}^{(0)}=0 \\
\tilde{R}^{(\mathrm{T})}=\int d t \tilde{\mathbf{v}}_{E} \cdot \nabla \rho=-2 i \Delta_{\rho} \frac{\tilde{\omega}_{E}}{\omega} \rho_{0} \sin \theta \\
\tilde{R}^{(\mathrm{P})}=\int d t \mathbf{V}_{P} \cdot \nabla \tilde{\rho}=i M_{P} \frac{k_{\|} c_{s}}{\omega} \frac{\partial \tilde{\rho}}{\partial \theta} .
\end{gathered}
$$




\section{E.2.4 Cálculo de $\nabla \cdot \tilde{\mathbf{q}}$}

Através da expressão para o fluxo de calor total,

$$
\mathbf{q}_{\Sigma}=\frac{\gamma}{\gamma-1} \frac{p_{\Sigma}}{e B^{2}}\left(\mathbf{B} \times \nabla T_{\Sigma}\right), \quad p_{\Sigma}=p+\tilde{p}, \quad T_{\Sigma}=T+\tilde{T}
$$

obtém-se o fluxo de calor perturbado em primeira ordem,

$$
\tilde{\mathbf{q}}=\frac{\tilde{p}}{p} \mathbf{q}+\frac{\gamma}{\gamma-1} \frac{p}{e B^{2}}(\mathbf{B} \times \nabla \tilde{T})
$$

e, consequentemente,

$$
\boldsymbol{\nabla} \cdot \tilde{\mathbf{q}}=\boldsymbol{\nabla} \cdot\left(\frac{\tilde{p}}{p} \mathbf{q}\right)+\frac{\gamma}{\gamma-1}\left[(\mathbf{B} \times \boldsymbol{\nabla} \tilde{T}) \cdot \boldsymbol{\nabla}\left(\frac{p}{e B^{2}}\right)+\frac{p}{e B^{2}}(\boldsymbol{\nabla} \times \mathbf{B}) \cdot \boldsymbol{\nabla} \tilde{T}\right]
$$

Como $\boldsymbol{\nabla} \cdot \mathbf{q}=\mathcal{O}\left(\varepsilon M_{\mathrm{th}} p_{0} k_{\|} c_{s}\right)$ é um termo de segunda ordem, segue que

$$
\boldsymbol{\nabla} \cdot\left(\frac{\tilde{p}}{p} \mathbf{q}\right) \approx \mathbf{q} \cdot \nabla\left(\frac{\tilde{p}}{p}\right) \approx \frac{\gamma}{\gamma-1} \frac{R_{0}^{2}}{e F_{0}} \mathbf{B} \cdot \nabla \tilde{p} \approx \frac{\gamma}{\gamma-1} M_{\mathrm{th}} k_{\|} c_{s} \frac{\partial \tilde{p}}{\partial \theta}
$$

De forma similar, os outros termos de (E.29) podem ser desenvolvidos, resultando nas relações

$$
\begin{gathered}
(\mathbf{B} \times \boldsymbol{\nabla} \tilde{T}) \cdot \boldsymbol{\nabla}\left(\frac{p}{e B^{2}}\right) \approx \frac{R_{0}^{2}}{e F_{0}} \frac{d p}{d \Psi} \mathbf{B} \cdot \boldsymbol{\nabla} \tilde{T}=M_{\mathrm{th}} k_{\|} c_{s} p_{0} \frac{\partial}{\partial \theta}\left(\frac{\tilde{T}}{T_{0}}\right) \sim \nabla \cdot\left(\frac{\tilde{p}}{p} \mathbf{q}\right), \\
\frac{p}{e B^{2}}(\boldsymbol{\nabla} \times \mathbf{B}) \cdot \boldsymbol{\nabla} \tilde{T} \approx \frac{p_{0} R_{0}^{2}}{e F_{0}^{2}} \frac{d F_{0}}{d \Psi} \mathbf{B} \cdot \nabla \tilde{T}=\mathcal{R}_{F} M_{\mathrm{th}} k_{\|} c_{s} \frac{\partial}{\partial \theta}\left(\frac{\tilde{T}}{T_{0}}\right)=\mathcal{O}\left(\mathcal{B}_{0}\right) \nabla \cdot\left(\frac{\tilde{p}}{p} \mathbf{q}\right) .
\end{gathered}
$$

O uso da relação aproximada entre a pressão, densidade e temperatura perturbadas,

$$
\tilde{p} \approx p_{0} \frac{\tilde{T}}{T_{0}}+p_{0} \frac{\tilde{\rho}}{\rho_{0}}
$$

permite combinar as expressões em (E.30) e (E.31) e obter a expressão final para $\boldsymbol{\nabla} \cdot \tilde{\mathbf{q}}$ :

$$
\boldsymbol{\nabla} \cdot \tilde{\mathbf{q}}=\frac{M_{\mathrm{th}} k_{\|} c_{s}}{\gamma-1}\left(2 \gamma \frac{\partial \tilde{p}}{\partial \theta}-c_{s}^{2} \frac{\partial \tilde{\rho}}{\partial \theta}\right)
$$




\section{E.2.5 Cálculo de $\tilde{P}$}

Ao assumirmos que $\lim _{M_{P} \rightarrow 0} M_{\mathrm{th}}=0$, obtém-se, analogamente cálculo de $\tilde{R}$, que:

$$
\begin{gathered}
\tilde{P}^{(\mathrm{T})}=\int d t \tilde{\mathbf{v}}_{E} \cdot \boldsymbol{\nabla} p=-2 i \frac{\Delta_{p}}{\gamma} \frac{\tilde{\omega}_{E}}{\omega} \rho_{0} c_{s}^{2} \sin \theta \\
\tilde{P}^{(\mathrm{P})}=\int d t\left[\mathbf{V}_{P} \cdot \nabla \tilde{p}+(\gamma-1) \nabla \cdot \tilde{\mathbf{q}}\right]=i \frac{k_{\|} c_{s}}{\omega}\left[\left(M_{P}+2 \gamma M_{\mathrm{th}}\right) \frac{\partial \tilde{p}}{\partial \theta}-M_{\mathrm{th}} c_{s}^{2} \frac{\partial \tilde{\rho}}{\partial \theta}\right] .
\end{gathered}
$$

\section{E.3 Solução sem rotação (primeira iteração)}

Com as substituições $\tilde{F}_{\|}=\tilde{R}=\tilde{P}=0$ e $\tilde{\mathbf{v}}=\tilde{\mathbf{v}}^{(0)}=\tilde{\mathbf{v}}_{E}+\tilde{v}_{\|}^{(0)} \mathbf{b}$ em (E.1) - (E.3) e o uso da normalização

$$
\Omega=\frac{\omega}{k_{\|} c_{s}}, \quad \tilde{\Omega}_{E}=\frac{\tilde{\omega}_{E}}{k_{\|} c_{s}}
$$

conforme explicitado na seção 3.4, obtém-se:

$$
\begin{gathered}
\frac{\boldsymbol{\nabla} \cdot \tilde{\mathbf{v}}^{(0)}}{k_{\|} c_{s}}=-2 \tilde{\Omega}_{E} \sin \theta+\frac{\partial}{\partial \theta} \frac{\tilde{v}_{\|}^{(0)}}{c_{s}}, \\
\tilde{v}_{\|}^{(0)}=\tilde{v}_{\| c}^{(0)} \cos \theta, \quad \tilde{v}_{\| c}^{(0)}=\frac{2 \tilde{\Omega}_{E}}{\Omega^{2}-1} c_{s}, \\
\frac{\tilde{\rho}_{s}^{(0)}}{\rho_{0}}=\frac{\tilde{p}_{s}^{(0)}}{\rho_{0} c_{s}^{2}}=i \Omega \frac{\tilde{v}_{\| c}^{(0)}}{c_{s}}, \quad \tilde{\rho}_{c}^{(0)}=\tilde{p}_{c}^{(0)}=0 .
\end{gathered}
$$

\section{E.4 Solução com rotação toroidal (segunda iteração)}


Desconsiderando em (E.1)-(E.3) a solução obtida em na seção anterior, obtemos o sistema:

$$
\begin{gathered}
-i \Omega \frac{\tilde{v}_{\|}^{(\mathrm{T})}}{c_{s}}+\frac{\partial}{\partial \theta} \frac{\tilde{p}^{(\mathrm{T})}}{\rho_{0} c_{s}^{2}}+\frac{\tilde{F}_{\|}^{(\mathrm{T})}}{\rho_{0} k_{\|} c_{s}^{2}}=0, \\
-i \Omega\left(\frac{\tilde{\rho}^{(\mathrm{T})}}{\rho_{0}}+\frac{\tilde{R}^{(\mathrm{T})}}{\rho_{0}}\right)+\frac{\partial}{\partial \theta} \frac{\tilde{v}_{\|}^{(\mathrm{T})}}{c_{s}}=0, \\
-i \Omega\left(\frac{\tilde{p}^{(\mathrm{T})}}{\rho_{0} c_{s}^{2}}+\frac{\tilde{P}^{(\mathrm{T})}}{\rho_{0} c_{s}^{2}}\right)+\frac{\partial}{\partial \theta} \frac{\tilde{v}_{\|}^{(\mathrm{T})}}{c_{s}}=0,
\end{gathered}
$$

que apresenta como solução

$$
\begin{gathered}
\tilde{v}_{\|}^{(\mathrm{T})}=\frac{i}{2} \frac{\Omega}{\tilde{\Omega}_{E}}\left(\frac{\partial}{\partial \theta} \frac{\tilde{P}^{(\mathrm{T})}}{\rho_{0} c_{s}^{2}}-\frac{\tilde{F}_{\|}^{(\mathrm{T})}}{\rho_{0} k_{\|} c_{s}^{2}}\right) \tilde{v}_{\| c}^{(0)}=\tilde{v}_{\| s}^{(\mathrm{T})} \sin \theta+\tilde{v}_{\| c}^{(\mathrm{T})} \cos \theta \\
\tilde{\rho}^{(\mathrm{T})}=-\left[\frac{1}{2 \tilde{\Omega}_{E}}\left(\frac{\tilde{P}^{(\mathrm{T})}}{c_{s}^{2}}+\frac{\partial}{\partial \theta} \frac{\tilde{F}_{\|}^{(\mathrm{T})}}{k_{\|} c_{s}^{2}}\right) \frac{\tilde{v}_{\| c}^{(0)}}{c_{s}}+\tilde{R}^{(\mathrm{T})}\right]=\tilde{\rho}_{s}^{(\mathrm{T})} \sin \theta+\tilde{\rho}_{c}^{(\mathrm{T})} \cos \theta \\
\tilde{p}^{(\mathrm{T})}=\frac{1}{2 \tilde{\Omega}_{E}}\left(\Omega^{2} \tilde{P}^{(\mathrm{T})}+\frac{\partial}{\partial \theta} \frac{\tilde{F}_{\|}^{(\mathrm{T})}}{k_{\|}}\right) \frac{\tilde{v}_{\| c}^{(0)}}{c_{s}}=\tilde{p}_{s}^{(\mathrm{T})} \sin \theta+\tilde{p}_{c}^{(\mathrm{T})} \cos \theta
\end{gathered}
$$

Mediante o uso dos resultados anteriores em (E.44) - (E.46), segue, finalmente, que

$$
\begin{gathered}
\tilde{v}_{\| s}^{(\mathrm{T})}=i \Omega \frac{\left(M_{T}+\Delta_{V}\right)}{2} \tilde{v}_{\| c}^{(0)}, \quad \tilde{v}_{\| c}^{(\mathrm{T})}=\frac{\Delta_{p}}{\gamma} \tilde{v}_{\| c}^{(0)}, \\
\tilde{\rho}_{s}^{(\mathrm{T})}=\frac{i}{\Omega}\left[\frac{\Delta_{p}}{\gamma}+\left(\Omega^{2}-1\right) \Delta_{\rho}\right] \frac{\tilde{v}_{\| c}^{(0)}}{c_{s}} \rho_{0}, \quad \tilde{\rho}_{c}^{(\mathrm{T})}=\frac{\left(M_{T}+\Delta_{V}\right)}{2} \frac{\tilde{v}_{\| c}^{(0)}}{c_{s}} \rho_{0}, \\
\tilde{p}_{s}^{(\mathrm{T})}=i \Omega \frac{\Delta_{p}}{\gamma} \rho_{0} c_{s} \tilde{v}_{\| c}^{(0)}, \quad \tilde{p}_{c}^{(\mathrm{T})}=\tilde{\rho}_{c}^{(\mathrm{T})} c_{s}^{2} .
\end{gathered}
$$




\section{E.5 Rotação poloidal e toroidal (terceira iteração)}

Primeiramente, substitui-se (E.47)-(E.49) em (E.21), (E.26) e (E.36) para obter

$$
\begin{gathered}
\frac{\tilde{F}_{\|}^{(\mathrm{P})}}{\rho_{0} k_{\|} c_{s}^{2}}=M_{P} \frac{\partial}{\partial \theta} \frac{\tilde{v}_{\|}^{(\mathrm{P})}}{c_{s}}+M_{P}\left[-\left(1+\frac{\Delta_{p}}{\gamma}\right) \sin \theta+\frac{i \Omega}{2}\left(M_{T}+\Delta_{V}\right) \cos \theta\right] \frac{\tilde{v}_{\| c}^{(0)}}{c_{s}} . \\
\frac{\tilde{R}^{(\mathrm{P})}}{\rho_{0}}=\frac{i M_{P}}{\Omega} \frac{\partial}{\partial \theta} \frac{\tilde{\rho}^{(\mathrm{P})}}{\rho_{0}}-\frac{M_{P}}{\Omega^{2}}\left\{\frac{i}{2} \Omega\left(M_{T}+\Delta_{V}\right) \sin \theta+\left[\Omega^{2}+\frac{\Delta_{p}}{\gamma}+\left(\Omega^{2}-1\right) \Delta_{\rho}\right] \cos \theta\right\} \frac{\tilde{v}_{\| c}^{(0)}}{c_{s}},(\mathrm{E} .5 \\
\frac{\tilde{P}^{(\mathrm{P})}}{\rho_{0} c_{s}^{2}}=i \frac{M_{P}}{\Omega} \frac{\partial}{\partial \theta}\left[\left(1+2 \gamma \frac{M_{\mathrm{th}}}{M_{P}}\right) \frac{\tilde{p}^{(\mathrm{P})}}{\rho_{0} c_{s}^{2}}-\frac{M_{\mathrm{th}}}{M_{P}} \frac{\tilde{\rho}^{(\mathrm{P})}}{\rho_{0}}\right]+\frac{M_{P}}{\Omega}\left\{i\left[1+(2 \gamma-1) \frac{M_{\mathrm{th}}}{M_{P}}\right] \sin \theta+\right. \\
\left.\left[\Omega \frac{\Delta_{p}}{\gamma}+\left(2 \gamma \Omega-\frac{1}{\Omega}\right) \frac{M_{\mathrm{th}}}{M_{P}}+2 \Omega \Delta_{p} \frac{M_{\mathrm{th}}}{M_{P}}+\left(\frac{1}{\Omega}-\Omega\right) \Delta_{\rho} \frac{M_{\mathrm{th}}}{M_{P}}\right] \cos \theta\right\} \frac{\tilde{v}_{\| c}^{(0)}}{c_{s}}, \quad(\mathrm{E} .52
\end{gathered}
$$

que, por sua vez, são inseridos no sistema:

$$
\begin{gathered}
\tilde{v}_{\|}^{(\mathrm{P})}=\frac{i \Omega}{\Omega^{2}-1}\left(\frac{\partial}{\partial \theta} \frac{\tilde{P}^{(\mathrm{P})}}{\rho_{0} c_{s}}-\frac{\tilde{F}_{\|}^{(\mathrm{P})}}{\rho_{0} k_{\|} c_{s}}\right), \\
\tilde{\rho}^{(\mathrm{P})}=-\tilde{R}^{(\mathrm{P})}-\frac{1}{\Omega^{2}-1}\left(\frac{\tilde{P}^{(\mathrm{P})}}{c_{s}^{2}}+\frac{\partial}{\partial \theta} \frac{\tilde{F}_{\|}^{(\mathrm{P})}}{k_{\|} c_{s}^{2}}\right), \\
\tilde{p}^{(\mathrm{P})}=-\frac{1}{\Omega^{2}-1}\left(\Omega^{2} \tilde{P}^{(\mathrm{P})}+\frac{\partial}{\partial \theta} \frac{\tilde{F}_{\|}^{(\mathrm{P})}}{k_{\|}}\right),
\end{gathered}
$$

cujas equações são similares às eqs. (E.44)-(E.46).

Para resolver este sistema, devido ao acoplamento das equações, é conveniente utilizar a formulação exponencial ao invés da trigonométrica, de acordo com as relações:

$$
\begin{array}{r}
\tilde{X}=\tilde{X}_{s} \sin \theta+\tilde{X}_{c} \cos \theta=\tilde{X}_{+1} \mathrm{e}^{i \theta}+\tilde{X}_{-1} \mathrm{e}^{-i \theta} \\
\tilde{X}_{s}=i\left(\tilde{X}_{+1}-\tilde{X}_{-1}\right), \quad \tilde{X}_{c}=\tilde{X}_{+1}+\tilde{X}_{-1}, \quad \tilde{X}_{ \pm 1}=\frac{1}{2}\left(\tilde{X}_{c} \mp i \tilde{X}_{s}\right) .
\end{array}
$$


A forma exponencial é adotada por duas razões: Primeiramente, devido à praticidade no cálculo da derivada, ou seja, $\partial / \partial \theta \rightarrow i m, m= \pm 1$. A segunda é devido a possibilidade de análise individual dos harmônicos $m=1,-1$ que a forma exponencial proporciona.

Nesta condições, de acordo com (E.21), (E.26) e (E.36), utilizando (E.56), segue que

$$
\begin{gathered}
\frac{\tilde{F}_{\| \pm 1}^{(\mathrm{P})}}{\rho_{0} k_{\|} c_{s}}= \pm i M_{P} \frac{\tilde{v}_{\| \pm 1}^{(\mathrm{P})}}{c_{s}}+\frac{i}{2} M_{P}\left[ \pm \frac{\left(M_{T}+\Delta_{V}\right)}{2} \Omega+1-\frac{\Delta_{p}}{\gamma}\right] \frac{\tilde{v}_{\| c}^{(0)}}{c_{s}} \\
\frac{\tilde{R}_{ \pm 1}^{(\mathrm{P})}}{\rho_{0}}=\mp \frac{M_{P}}{\Omega} \frac{\tilde{\rho}_{ \pm}^{(\mathrm{P})}}{\rho_{0}}-\frac{M_{P}}{2}\left[1+\Delta_{\rho} \pm \frac{1}{2} \frac{\left(M_{T}+\Delta_{V}\right)}{\Omega}+\frac{\left(\Delta_{p} / \gamma-\Delta_{\rho}\right)}{\Omega^{2}}\right] \frac{\tilde{v}_{\| c}^{(0)}}{c_{s}} \\
\frac{\tilde{P}_{ \pm 1}^{(\mathrm{P})}}{\rho_{0} c_{s}^{2}}=\mp \frac{M_{P}}{\Omega}\left[\left(1+2 \gamma \frac{M_{\mathrm{th}}}{M_{P}}\right) \frac{\tilde{p}_{ \pm 1}^{(\mathrm{P})}}{\rho_{0} c_{s}^{2}}-\frac{M_{\mathrm{th}}}{M_{P}} \frac{\tilde{\rho}_{ \pm 1}^{(\mathrm{P})}}{\rho_{0}}\right]- \\
\frac{M_{P}}{2}\left\{1+\frac{\Delta_{p}}{\gamma}+(2 \gamma-1) \frac{M_{\mathrm{th}}}{M_{P}} \pm \frac{1}{2}\left[1+(2 \gamma-1) \frac{M_{\mathrm{th}}}{M_{P}}\right] \frac{\left(M_{T}+\Delta_{V}\right)}{\Omega}\right\} \frac{\tilde{v}_{\| c}^{(0)}}{c_{s}}
\end{gathered}
$$

e, com a substituição de (E.57), (E.58) e (E.59) em (E.53), (E.54) e (E.55), obtemos um sistema $6 \times 6$, que pode ser representado na forma matricial:

$$
\left(\begin{array}{ccc}
\mathcal{C}_{11}^{( \pm)} & \mathcal{C}_{12} & \mathcal{C}_{13} \\
\mathcal{C}_{21} & \mathcal{C}_{22}^{( \pm)} & \mathcal{C}_{23}^{( \pm)} \\
\mathcal{C}_{31} & \mathcal{C}_{32}^{( \pm)} & \mathcal{C}_{33}^{( \pm)}
\end{array}\right)\left(\begin{array}{c}
\tilde{v}_{\| \pm 1}^{(\mathrm{P})} / c_{s} \\
\tilde{\rho}_{ \pm 1}^{(\mathrm{P})} / \rho_{0} \\
\tilde{p}_{ \pm 1}^{(\mathrm{P})} / \rho_{0} c_{s}^{2}
\end{array}\right)=\frac{1}{2} \frac{M_{P} \tilde{v}_{\| c}^{(0)}}{\Omega^{2}-1}\left(\begin{array}{c}
\mathcal{K}_{v}^{( \pm)} \\
\mathcal{K}_{\rho}^{( \pm)} \\
\mathcal{K}_{p}^{( \pm)}
\end{array}\right)
$$

onde

$$
\begin{aligned}
& \mathcal{C}_{11}^{( \pm)}=1 \pm \frac{\mathcal{C}_{31} \Omega}{\Omega^{2}-1}, \quad \mathcal{C}_{12}=\frac{M_{\mathrm{th}}}{\Omega^{2}-1}, \quad \mathcal{C}_{13}=\mathcal{C}_{21}+2 \gamma \mathcal{C}_{12} \\
& \mathcal{C}_{21}=\frac{-\mathcal{C}_{31}}{\Omega^{2}-1}, \quad \mathcal{C}_{22}^{( \pm)}=1 \pm\left(\frac{\mathcal{C}_{12}+\mathcal{C}_{31}}{\Omega}\right), \quad \mathcal{C}_{23}^{( \pm)}=\mp \frac{\mathcal{C}_{13}}{\Omega} \\
& \mathcal{C}_{31}=-M_{P}, \quad \mathcal{C}_{32}^{( \pm)}= \pm \mathcal{C}_{12} \Omega, \quad \mathcal{C}_{33}^{( \pm)}=1 \mp \mathcal{C}_{13} \Omega
\end{aligned}
$$




$$
\begin{aligned}
& \mathcal{K}_{v}^{( \pm)}= \pm \frac{\left(M_{T}+\Delta_{V}\right)}{2} \Omega^{2}+\left[1-\frac{\Delta_{p}}{\gamma} \pm\left(1+\frac{\Delta_{p}}{\gamma}+(2 \gamma-1) \frac{M_{\mathrm{th}}}{M_{P}}\right)\right] \Omega+ \\
& \frac{\left(M_{T}+\Delta_{V}\right)}{2}\left(1+(2 \gamma-1) \frac{M_{\mathrm{th}}}{M_{P}}\right) \\
& \mathcal{K}_{\rho}^{( \pm)}=\left(1+\Delta_{\rho}\right) \Omega^{2}+(1 \pm 1) \frac{\left(M_{T}+\Delta_{V}\right)}{2} \Omega \pm\left(1-\frac{\Delta_{p}}{\gamma}\right)+2\left(\frac{\Delta_{p}}{\gamma}-\Delta_{\rho}\right)+ \\
& (2 \gamma-1) \frac{M_{\mathrm{th}}}{M_{P}} \pm(2 \gamma-1) \frac{\left(M_{T}+\Delta_{V}\right)}{2} \frac{M_{\mathrm{th}}}{M_{P}} \frac{1}{\Omega}+\left(\Delta_{\rho}-\frac{\Delta_{p}}{\gamma}\right) \frac{1}{\Omega^{2}}, \\
& \mathcal{K}_{p}^{( \pm)}=\left(1+\frac{\Delta_{p}}{\gamma}+(2 \gamma-1) \frac{M_{\mathrm{th}}}{M_{P}}\right) \Omega^{2}+\frac{\left(M_{T}+\Delta_{V}\right)}{2}\left(1 \pm 1 \pm(2 \gamma-1) \frac{M_{\mathrm{th}}}{M_{P}}\right) \Omega \\
& \pm\left(1-\frac{\Delta_{p}}{\gamma}\right) .
\end{aligned}
$$

A solução deste sistema pode ser representada pelas seguintes relações:

$$
\frac{\tilde{v}_{\| \pm 1}^{(\mathrm{P})}}{c_{s}}=\frac{\mathcal{N}_{ \pm 1}^{v}(\mathrm{P})}{\mathcal{D}_{ \pm 1}(\mathrm{P})}, \quad \frac{\tilde{\rho}_{ \pm 1}^{(\mathrm{P})}}{\rho_{0}}=\frac{\mathcal{N}_{ \pm 1}^{\rho}(\mathrm{P})}{\mathcal{D}_{ \pm 1}(\mathrm{P})}, \quad \frac{\tilde{p}_{ \pm 1}^{(\mathrm{P})}}{\rho_{0} c_{s}^{2}}=\frac{\mathcal{N}_{ \pm 1}^{p}(\mathrm{P})}{\mathcal{D}_{ \pm 1}(\mathrm{P})}
$$

onde

$$
\begin{gathered}
\mathcal{D}_{ \pm 1}{ }^{(\mathrm{P})} \approx\left(M_{P} \mp \Omega\right)\left(\Omega+1 \mp M_{P}\right)\left(\Omega-1 \mp M_{P}\right)+\left[2 \gamma\left(\Omega \mp M_{P}\right)^{2}-1\right] M_{\mathrm{th}} \\
\mathcal{N}_{ \pm 1}^{v}{ }^{(\mathrm{P})}=M_{P} \sum_{k=0}^{3} \mathcal{C}_{k, \pm 1}^{(v)} \Omega^{k}, \quad \mathcal{C}_{0, \pm 1}^{(v)}=\frac{M_{P}}{2}\left(-M_{P}+\frac{M_{T}}{2}+\frac{M_{P} \Delta_{V}}{2}\right), \\
\mathcal{C}_{1, \pm 1}^{(v)}=\mp \frac{1}{2}\left\{\left(1+M_{P}^{2}\right) \frac{\left(M_{T}+\Delta_{V}\right)}{2}-3 M_{P}\left(1+\frac{\Delta_{p}}{\gamma}\right)+\left[(2 \gamma-1) \frac{\left(M_{T}+\Delta_{V}\right)}{2 M_{P}}-4 \gamma\right] M_{\mathrm{th}}\right\} \\
\mathcal{C}_{2, \pm 1}^{(v)}=-\left(1+\frac{\Delta_{p}}{\gamma}\right)+\frac{\left(M_{T}+\Delta_{V}\right)}{2} M_{P}-\left(\gamma-\frac{1}{2}\right) \frac{M_{\mathrm{th}}}{M_{P}}, \quad \mathcal{C}_{3, \pm 1}^{(v)}=\mp \frac{\left(M_{T}+\Delta_{V}\right)}{4}, \\
\mathcal{N}_{ \pm 1}^{\rho}(\mathrm{P})=\frac{M_{P}}{\Omega} \sum_{k=0}^{4} \mathcal{C}_{k, \pm 1}^{(\rho)} \Omega^{k}, \quad \mathcal{C}_{0, \pm 1}^{(\rho)}=\mp \frac{1}{2}\left(\Delta_{\rho}-\frac{\Delta_{p}}{\gamma}\right) \\
\mathcal{C}_{1, \pm 1}^{(\rho)}=\frac{M_{P}}{2}\left[1-2 \Delta_{\rho}+3 \frac{\Delta_{p}}{\gamma}-M_{P} \frac{\left(M_{T}+\Delta_{V}\right)}{2}\right]+\left[1-(2 \gamma-1) \frac{\left(M_{T}+\Delta_{V}\right)}{4 M_{P}}\right] M_{\mathrm{th}} \\
\mathcal{C}_{2, \pm 1}^{(\rho)}=\mp \frac{1}{2}\left[1-2 \Delta_{\rho}+3 \frac{\Delta_{p}}{\gamma}-\frac{3}{2} M_{P}\left(M_{T}+\Delta_{V}\right)+M_{P}^{2}+(2 \gamma-1) \frac{M_{\mathrm{th}}}{M_{P}}\right] \\
\mathcal{C}_{3, \pm 1}^{(\rho)}=-\frac{M_{T}+\Delta_{V}}{2}+\left(1+\Delta_{\rho}\right) M_{P}+\gamma M_{\mathrm{th}}, \quad \mathcal{C}_{4, \pm 1}^{(\rho)}=\mp \frac{1}{2}\left(1+\Delta_{\rho}\right),
\end{gathered}
$$




$$
\begin{aligned}
& \mathcal{N}_{ \pm 1}^{p(\mathrm{P})}=M_{P} \sum_{k=0}^{3} \mathcal{C}_{k, \pm 1}^{(\rho)} \Omega^{k}, \quad \mathcal{C}_{0, \pm 1}^{(p)}=\frac{M_{P}}{2}\left[1+\frac{\Delta_{p}}{\gamma}-\frac{\left(M_{T}+\Delta_{V}\right)}{2} M_{P}\right]+\frac{M_{\mathrm{th}}}{2} \\
& \mathcal{C}_{1, \pm 1}^{(p)}=\mp \frac{1}{2}\left(1+\frac{\Delta_{p}}{\gamma}-\frac{3}{2}\left(M_{T}+\Delta_{V}\right) M_{P}+M_{P}^{2}\right) \\
& \mathcal{C}_{2, \pm 1}^{(p)}=\left(1+\frac{\Delta_{p}}{\gamma}\right) M_{P}-\frac{\left(M_{T}+\Delta_{V}\right)}{2}+\left[2 \gamma-\frac{1}{2}-(2 \gamma-1) \frac{\left(M_{T}+\Delta_{V}\right)}{4 M_{P}}\right] M_{\mathrm{th}} \\
& \mathcal{C}_{3, \pm 1}^{(p)}=\mp \frac{1}{2}\left(1+\frac{\Delta_{p}}{\gamma}+(2 \gamma-1) \frac{M_{\mathrm{th}}}{M_{P}}\right) .
\end{aligned}
$$

\section{E.6 Relação de dispersão}

Para a obtenção da relação de dispersão é necessário invocar a equação do momento,

$$
\rho \frac{\partial \tilde{\mathbf{v}}}{\partial t}+\nabla \tilde{p}-\tilde{\mathbf{J}} \times \mathbf{B}+\tilde{\mathbf{F}}=0, \quad \tilde{\mathbf{F}}=\rho(\mathbf{V} \cdot \nabla \tilde{\mathbf{v}}+\tilde{\mathbf{v}} \cdot \nabla \mathbf{V})+\tilde{\rho} \mathbf{V} \cdot \nabla \mathbf{V}
$$

a qual, quando multiplicada vetorialmente por $\mathbf{B}$ permite a obtenção da expressão analítica para a densidade de corrente,

$$
\tilde{\mathbf{J}}=\frac{\tilde{j}_{\|}}{B} \mathbf{B}+\frac{\rho \mathbf{B}}{B^{2}} \times \frac{\partial \tilde{\mathbf{v}}}{\partial t}+\frac{\mathbf{B}}{B^{2}} \times \nabla \tilde{p}+\frac{\mathbf{B}}{B^{2}} \times \tilde{\mathbf{F}}
$$

A relação de dispersão é proveniente da condição de quasi-neutralidade do plasma, que pode ser expressa pela equação $\boldsymbol{\nabla} \cdot \tilde{\mathbf{J}}=0$. A metodologia analítica padrão é baseada no cálculo da média de tal equação sobre uma superfície magnética. De forma similar, podemos tomar a média da equação mencionada com relação a um volume arbitrário de plasma, ou seja,

$$
D=\frac{\int_{V} d V \boldsymbol{\nabla} \cdot \tilde{\mathbf{J}}}{\int_{V} d V}=0, \quad d V=\left(R_{0}+r \cos \theta\right) r d r d \theta d \phi
$$

O cálculo do numerador de $D$, em (E.72), é efetuado a partir do uso do teorema da divergência de Gauss, (B.5), de forma que

$$
D=\frac{\oint_{S} \mathbf{J} \cdot d \mathbf{S}}{\int_{V} d V}=0, \quad d \mathbf{S}=\left(R_{0}+r \cos \theta\right) r d \theta d \phi \hat{\mathbf{e}}_{r} .
$$




$$
\begin{aligned}
& D^{(0)} \approx \frac{K(r)}{2 i \pi}\left[-i \frac{\tilde{\Omega}_{E}}{q^{2}} \Omega \int_{0}^{2 \pi} d \theta+2 \int_{0}^{2 \pi} d \theta \frac{\partial}{\partial \theta}\left(\frac{\tilde{p}^{(0)}}{\rho_{0} c_{s}^{2}}\right) \cos \theta\right], \\
& D^{(\mathrm{T})} \approx \frac{K(r)}{2 i \pi}\left[2 \int_{0}^{2 \pi} d \theta \frac{\partial}{\partial \theta}\left(\frac{\tilde{p}^{(\mathrm{T})}}{\rho_{0} c_{s}^{2}}\right) \cos \theta+\frac{1}{q} \int_{0}^{2 \pi} d \theta \frac{\tilde{F}_{\theta}^{(\mathrm{T})}}{\rho_{0} k_{\|} c_{s}^{2}}\right], \\
& D^{(\mathrm{P})} \approx \frac{K(r)}{2 i \pi}\left[2 \int_{0}^{2 \pi} d \theta \frac{\partial}{\partial \theta}\left(\frac{\tilde{p}^{(\mathrm{P})}}{\rho_{0} c_{s}^{2}}\right) \cos \theta+\frac{1}{q} \int_{0}^{2 \pi} d \theta \frac{\tilde{F}_{\theta}^{(\mathrm{P})}}{\rho_{0} k_{\|} c_{s}^{2}}\right],
\end{aligned}
$$

onde $K(r) \approx 2 i \gamma p_{0}(r) / r F_{0}(r)$. Com a substituição dos resultados anteriores obtidos nesta seção nas expressões (E.74) - (E.76), obtemos os resultados mostrados em (3.87) - (3.89). 


\section{Apêndice F}

\section{Cálculo de integrais da função}

\section{distribuição}

\section{F.1 Relações envolvendo a distribuição maxwelliana}

Em problemas envolvendo a função distribuição Maxwelliana, a qual é definida por

$$
F_{M_{\alpha}}=\frac{n_{0}}{\pi^{3 / 2} v_{T_{\alpha}}^{3}} \exp \left(-\frac{v_{\perp}^{2}+v_{\|}^{2}}{v_{T_{\alpha}}^{2}}\right)
$$

é comum aparecer integrais do tipo:

$$
I(a, b)=\int_{0}^{2 \pi} d \gamma \int_{-\infty}^{\infty} d v_{\|} \int_{0}^{\infty} d v_{\perp} v_{\perp}\left(\frac{v_{\perp}}{v_{T_{\alpha}}}\right)^{a}\left(\frac{v_{\|}}{v_{T_{\alpha}}}\right)^{b} F_{M_{\alpha}}, \quad a, b \geq 0
$$

que podem ser simplificadas a partir das mudanças de variável: $x=v_{\perp} / v_{T_{\alpha}}$ e $y=v_{\|} / v_{T_{\alpha}}$,

$$
I(a, b)=\frac{2 n_{0}}{\sqrt{\pi}} I_{\perp}(a) I_{\|}(b)
$$

As soluções para $I_{\perp}(a)$ e $I_{\|}(b)$, de forma genérical, são dadas por:

$$
I_{\perp}(n)=\int_{0}^{\infty} x^{n+1} \mathrm{e}^{-x^{2}} d x= \begin{cases}2^{-1}(n / 2) ! & \text { para } n \text { par } \\ 2^{-(n+1) / 2} n ! ! \sqrt{\pi} & \text { para } n \text { impar }\end{cases}
$$




$$
I_{\|}(n)=\int_{-\infty}^{\infty} x^{n} \mathrm{e}^{-\alpha x^{2}} d x= \begin{cases}\sqrt{\pi} & \text { para } n=0 \\ 0 & \text { para } n \text { impar } \\ 2^{-n / 2}(n-1) ! ! \sqrt{\pi} & \text { para } n \geq 2 \text { e } n \text { par }\end{cases}
$$

A partir da energia e da velocidade térmica de partículas do tipo $\alpha$,

$$
\mathcal{E}_{\alpha}=e_{\alpha} \Phi+\frac{1}{2} m_{\alpha} v^{2}, \quad v_{T_{\alpha}}^{2}=\frac{2 T_{\alpha}}{m_{\alpha}}
$$

onde $v^{2}=v_{\perp}^{2}+v_{\|}^{2}$, podemos expressar a função Maxwelliana como:

$$
F_{M_{\alpha}}=\frac{n_{0} m_{\alpha}^{3 / 2}}{\left(2 \pi T_{\alpha}\right)^{3 / 2}} \exp \left(-\frac{\mathcal{E}_{\alpha}-e_{\alpha} \Phi}{T_{\alpha}}\right)
$$

Segue, portanto, que

$$
\begin{gathered}
\frac{\partial F_{M s}}{\partial \mathcal{E}_{\alpha}}=-\frac{F_{M s}}{T_{\alpha}}, \\
\nabla F_{M_{\alpha}}=\left[\nabla \ln n_{0}+\left(\frac{\mathcal{E}_{\alpha}-e \Phi}{T_{\alpha}}-\frac{3}{2}\right) \nabla \ln T_{\alpha}+\frac{e \boldsymbol{\nabla} \Phi}{T_{\alpha}}\right] F_{M_{\alpha}},
\end{gathered}
$$

Para o caso particular em que $\Phi=0, n_{0} \approx n_{0}(r)$ e $T_{\alpha} \approx T_{\alpha}(r)$, o qual consideramos nesta tese, a eq. (F.9) pode ser simplificada, resultando em:

$$
\nabla F_{M_{\alpha}} \approx \hat{\mathbf{e}}_{r} \frac{n_{0}}{L_{N}}\left[1+\eta_{\alpha}\left(\frac{v_{\perp}^{2}}{v_{T_{\alpha}}^{2}}+\frac{v_{\|}^{2}}{v_{T_{\alpha}}^{2}}-\frac{3}{2}\right)\right] F_{M_{\alpha}}
$$

onde

$$
L_{N}^{-1}=\frac{\partial \ln n_{0}}{\partial r}, \quad L_{T_{\alpha}}^{-1}=\frac{\partial \ln T_{\alpha}}{\partial r} \quad \text { e } \quad \eta_{\alpha}=\frac{L_{N}}{L_{T}} .
$$




\section{F.2 Cálculo das integrais na aproximação de fluido}

A partir da integral no espaço de velocidades da grandeza arbitrária, $X_{\alpha}=X_{\alpha}\left(\mathbf{r}, v_{\perp}, v_{\|}, \gamma\right)$, a qual é denotada por

$$
\left\langle X_{\alpha}\right\rangle=\frac{1}{n_{0}} \int_{v} d^{3} v F_{M_{\alpha}} X_{\alpha}=\frac{1}{\pi} \int_{0}^{2 \pi} d \gamma \int_{0}^{\infty} d v_{\perp} v_{\perp} \frac{\mathrm{e}^{-v_{\perp}^{2} / v_{T_{\alpha}}^{2}}}{v_{T_{\alpha}}^{2}} \frac{1}{\sqrt{\pi}} \int_{-\infty}^{\infty} d v_{\|} \frac{\mathrm{e}^{-v_{\|}^{2} / v_{T_{\alpha}}^{2}}}{v_{T_{\alpha}}} X_{\alpha}
$$

nesta seção mostramos os resultados das seguintes quantidades:

$$
I_{a}^{(\alpha)}=\left\langle J_{0 \alpha}^{2} \Omega_{\mathrm{d} \alpha}^{a}\right\rangle, \quad I_{a b}^{(\alpha)}=\left\langle J_{0 \alpha}^{2} \Omega_{\mathrm{d} \alpha}^{a} \Omega_{\mathrm{tr} \alpha}^{b}\right\rangle, \quad I_{a b c}^{(\alpha)}=\left\langle J_{0 \alpha}^{2} \Omega_{\mathrm{d} \alpha}^{a} \Omega_{\mathrm{tr} \alpha}^{b} \Omega_{* \alpha}^{c}\right\rangle,
$$

onde

$$
\begin{gathered}
J_{0 \alpha}=J_{0}\left(\mathrm{k}_{r} v_{\perp} / \omega_{c_{\alpha}}\right) \approx 1-\frac{1}{2} \frac{v_{\perp}^{2}}{v_{T_{\alpha}}^{2}} \mathrm{k}_{r}^{2} \rho_{\alpha}^{2}+\frac{3}{32} \frac{v_{\perp}^{4}}{v_{T_{\alpha}}^{4}} \mathrm{k}_{r}^{4} \rho_{\alpha}^{4}+\mathcal{O}\left(\mathrm{k}_{r}^{6} \rho_{\alpha}^{6}\right), \\
\Omega_{\mathrm{d} \alpha}=\frac{\omega_{\mathrm{d} \alpha}}{\omega}\left(\frac{1}{2} \frac{v_{\perp}^{2}}{v_{T_{\alpha}}^{2}}+\frac{v_{\|}^{2}}{v_{T_{\alpha}}^{2}}\right), \quad \Omega_{\operatorname{tr} \alpha}=\frac{\omega_{\operatorname{tr} \alpha}}{\omega} \frac{v_{\|}}{v_{T_{\alpha}}}, \quad \Omega_{* \alpha}=\frac{\omega_{* \alpha}}{\omega}\left[1+\eta_{\alpha}\left(\frac{1}{2} \frac{v_{\perp}^{2}}{v_{T_{\alpha}}^{2}}+\frac{v_{\|}^{2}}{v_{T_{\alpha}}^{2}}\right)\right] .
\end{gathered}
$$

Para o cálculo das integrais em (F.13), observando que $\Omega_{\mathrm{d} \alpha}, \Omega_{\operatorname{tr} \alpha}$ e $\Omega_{* \alpha}$ são independentes de $\gamma$, é conveniente, primeiramente integrar nesta variável, posteriormente em $v_{\perp}$ e, por fim, em $v_{\|}$. A partir de (F.4), (F.5) e das mudanças de variáveis introduzidas anteriormente, $v_{\perp} / v_{T_{\alpha}}=x$ e $v_{\|} / v_{T_{\alpha}}=y$, para $\mathrm{k}_{r} \rho_{i} \ll 1$, em termos das frequências normalizadas, $\Omega=\omega R_{0} / v_{T_{i}}$ e $\Omega_{* \alpha}=$ $\omega_{* \alpha} R_{0} / v_{T_{i}}$, são obtidos os seguintes resultados:

$$
\begin{aligned}
& I_{0}^{(\alpha)}=\left\langle J_{0 \alpha}^{2}\right\rangle=1-\frac{1}{2} \mathrm{k}_{r}^{2} \rho_{\alpha}^{2}+\frac{3}{16} \mathrm{k}_{r}^{4} \rho_{\alpha}^{4}, \\
& I_{1}^{(\alpha)}=\left\langle J_{0 \alpha}^{2} \Omega_{\mathrm{d} \alpha}\right\rangle \approx\left(1-\frac{3}{4} \mathrm{k}_{r}^{2} \rho_{\alpha}^{2}\right) \frac{\mathrm{k}_{r} \rho_{\alpha}}{\Omega}, \\
& I_{2}^{(\alpha)}=\left\langle J_{0 \alpha}^{2} \Omega_{\mathrm{d} \alpha}^{2}\right\rangle \approx\left(\frac{7}{4}-\frac{13}{8} \mathrm{k}_{r}^{2} \rho_{\alpha}^{2}\right) \frac{\mathrm{k}_{r}^{2} \rho_{\alpha}^{2}}{\Omega^{2}},
\end{aligned}
$$




$$
\begin{gathered}
I_{02}^{(\alpha)}=\left\langle J_{0 \alpha}^{2} \Omega_{\mathrm{tr} \alpha}^{2}\right\rangle=\left(\frac{1}{2}-\frac{1}{4} \mathrm{k}_{r}^{2} \rho_{\alpha}^{2}\right) \frac{1}{q^{2} \Omega^{2}}, \\
I_{12}^{(\alpha)}=\left\langle J_{0 \alpha}^{2} \Omega_{\mathrm{d} \alpha} \Omega_{\mathrm{tr} \alpha}^{2}\right\rangle=\left(1-\frac{5}{8} \mathrm{k}_{r}^{2} \rho_{\alpha}^{2}\right) \frac{\mathrm{k}_{r} \rho_{\alpha}}{q^{2} \Omega^{3}} \\
I_{22}^{(\alpha)}=\left\langle J_{0 \alpha}^{2} \Omega_{\mathrm{d} \alpha}^{2} \Omega_{\mathrm{tr} \alpha}^{2}\right\rangle=\left(\frac{23}{8}-\frac{23}{16} \mathrm{k}_{r}^{2} \rho_{\alpha}^{2}\right) \frac{\mathrm{k}_{r}^{2} \rho_{\alpha}^{2}}{q^{2} \Omega^{4}} \\
I_{001}^{(\alpha)}=\left\langle J_{0 \alpha}^{2} \Omega_{* \alpha}\right\rangle=\left[1-\frac{\left(1+\eta_{\alpha}\right)}{2} \mathrm{k}_{r}^{2} \rho_{\alpha}^{2}\right] \frac{\Omega_{* \alpha}}{\Omega}, \\
I_{101}^{(\alpha)}=\left\langle J_{0 \alpha}^{2} \Omega_{\mathrm{d} \alpha} \Omega_{* \alpha}\right\rangle=\left[1+\eta_{\alpha}-\frac{3}{4}\left(1+2 \eta_{\alpha}\right) \mathrm{k}_{r}^{2} \rho_{\alpha}^{2}\right] \frac{\Omega_{* \alpha} \mathrm{k}_{r} \rho_{\alpha}}{\Omega^{2}} \\
I_{121}^{(\alpha)}=\left\langle J_{0 \alpha}^{2} \Omega_{\mathrm{d} \alpha} \Omega_{\mathrm{tr} \alpha}^{2} \Omega_{* \alpha}\right\rangle=\left[1+2 \eta_{\alpha}-\frac{5}{8}\left(1+3 \eta_{\alpha}\right) \mathrm{k}_{r}^{2} \rho_{\alpha}^{2}\right] \frac{\Omega_{* \alpha} \mathrm{k}_{r} \rho_{\alpha}}{q^{2} \Omega^{4}}, \\
I_{021}^{(\alpha)}=\left\langle J_{0 \alpha}^{2} \frac{\Omega_{\mathrm{tr} \alpha}^{2} \Omega_{* \alpha}}{\omega^{3}}\right\rangle=\left[\frac{1}{2}\left(1+\eta_{\alpha}\right)-\frac{1}{4}\left(1+2 \eta_{\alpha}\right) \mathrm{k}_{r}^{2} \rho_{\alpha}^{2}\right] \frac{\Omega_{* \alpha}}{q^{2} \Omega^{3}}
\end{gathered}
$$

\section{F.3 Função dispersão de plasma}

Para a função dispersão de plasma,

$$
Z(\zeta)=\frac{1}{\sqrt{\pi}} \int_{-\infty}^{\infty} d x \frac{\mathrm{e}^{-x^{2}}}{x-\zeta}, \quad \mathcal{I} m(\zeta)>0
$$

são satisfeitas as seguintes propriedades:

$$
\begin{gathered}
Z(-\zeta)=2 i \sqrt{\pi} \mathrm{e}^{-\zeta^{2}}-Z(\zeta), \\
\frac{d Z}{d \zeta}=-2[1+\zeta Z(\zeta)] .
\end{gathered}
$$

Se $|\mathcal{I} m(\zeta)| \ll 1$, mediante o prolongamento analítico para incluir o caso $\mathcal{I} m<0$, as seguintes aproximações assintóticas podem ser feitas:

$$
Z(\zeta) \approx i \sqrt{\pi} \mathrm{e}^{-\zeta^{2}}-2 \zeta+\frac{4}{3} \zeta^{3}+\mathcal{O}\left(\zeta^{5}\right)
$$


para $|\zeta| \ll 1 \mathrm{e}$

$$
Z(\zeta) \approx i \sigma \sqrt{\pi} \mathrm{e}^{-\zeta^{2}}-\left[\frac{1}{\zeta}+\frac{1}{2 \zeta^{3}}+\frac{3}{4 \zeta^{5}}+\frac{15}{8 \zeta^{7}}+\mathcal{O}\left(\zeta^{-9}\right)\right], \quad \sigma=\left\{\begin{array}{cc}
0 & \text { se } \operatorname{I} m(\zeta)>0 \\
1 & \text { se } \mathcal{I} m(\zeta)=0 \\
2 & \text { se } \operatorname{Im}(\zeta)<0
\end{array}\right.
$$

para $|\zeta| \gg 1$

Para simplificar a notação, definimos $Z^{(k)}=d^{k} Z / d \zeta^{k}$. Para os cálculos que se seguem, é conveniente, a partir de (F.22), calcular as seguintes derivadas:

$$
\begin{aligned}
& Z^{(1)}=-2-2 \zeta Z, \\
& Z^{(2)}=4 \zeta+\left(-2+4 \zeta^{2}\right) Z, \\
& Z^{(3)}=8-8 \zeta^{2}+\left(12 \zeta-8 \zeta^{3}\right) Z, \\
& Z^{(4)}=-40 \zeta+16 \zeta^{3}+\left(12-48 \zeta^{2}+16 \zeta^{4}\right) Z, \\
& Z^{(5)}=-64+144 \zeta^{2}-32 \zeta^{4}+\left(-120 \zeta+160 \zeta^{3}-32 \zeta^{5}\right) Z,
\end{aligned}
$$

Com relação à seguinte função relacionada a $Z(\zeta)$,

$$
Z_{n}(\zeta)=\frac{1}{\sqrt{\pi}} \int_{-\infty}^{\infty} d \zeta \frac{x^{n} \mathrm{e}^{-x^{2}}}{x-\zeta}, \quad n \geq 0
$$

utilizando (F.25), obtemos os seguintes resultados:

$$
\begin{aligned}
& Z_{0}(\zeta)=Z(\zeta) \\
& Z_{1}(\zeta)=-\frac{1}{2} Z^{(1)}=1+\zeta Z(\zeta) \\
& Z_{2}(\zeta)=\frac{1}{4}\left[2 Z+Z^{(2)}\right]=\zeta+\zeta^{2} Z(\zeta) \\
& Z_{3}(\zeta)=-\frac{1}{8}\left[6 Z^{(1)}+Z^{(3)}\right]=\frac{1}{2}+\zeta^{2}+\zeta^{3} Z(\zeta) \\
& Z_{4}(\zeta)=\frac{1}{16}\left[12 Z+12 Z^{(2)}+Z^{(4)}\right]=\frac{1}{2} \zeta+\zeta^{3}+\zeta^{4} Z(\zeta) \\
& Z_{5}(\zeta)=-\frac{1}{32}\left[60 Z^{(1)}(\zeta)+20 Z^{(3)}(\zeta)+Z^{(5)}(\zeta)\right]=\frac{3}{4}+\frac{1}{2} \zeta^{2}+\zeta^{4}+\zeta^{5} Z(\zeta)
\end{aligned}
$$

Finalmente, é conveniente definir a seguinte função diferença:

$$
D_{n}\left(\zeta_{\alpha}\right)=Z_{n}\left(-\zeta_{\alpha}\right)-Z_{n}\left(\zeta_{\alpha}\right), \quad \zeta_{\alpha}=\frac{\omega}{\omega_{\operatorname{tr} \alpha}}
$$


a qual, pela utilização de (F.21)-(F.27), resulta nos seguintes valores:

$$
\begin{aligned}
& D_{0}\left(\zeta_{\alpha}\right)=2\left[i \sqrt{\pi} \mathrm{e}^{-\zeta_{\alpha}^{2}}-Z\left(\zeta_{\alpha}\right)\right], \\
& D_{1}\left(\zeta_{\alpha}\right)=-2 \zeta_{\alpha} i \sqrt{\pi} \mathrm{e}^{-\zeta_{\alpha}^{2}} \\
& D_{2}\left(\zeta_{\alpha}\right)=-2 \zeta_{\alpha}+\zeta_{\alpha}^{2} D_{0}\left(\zeta_{\alpha}\right), \\
& D_{3}\left(\zeta_{\alpha}\right)=-2 \zeta_{\alpha}^{3} i \sqrt{\pi} \mathrm{e}^{-\zeta_{\alpha}^{2}} \\
& D_{4}\left(\zeta_{\alpha}\right)=-\zeta_{\alpha}-2 \zeta_{\alpha}^{3}+\zeta_{\alpha}^{4} D_{0}\left(\zeta_{\alpha}\right), \\
& D_{5}\left(\zeta_{\alpha}\right)=-2 \zeta_{\alpha}^{5} i \sqrt{\pi} \mathrm{e}^{-\zeta_{\alpha}^{2}}
\end{aligned}
$$

\section{F.4 Cálculo das integrais com efeitos cinéticos}

Inicialmente apresentamos as integrais cinéticas:

$$
\mathcal{L}_{a}^{(\alpha)}=\left\langle\frac{J_{0 \alpha}^{2} \Omega_{\mathrm{d} \alpha}^{a}}{1-\Omega_{\mathrm{tr} \alpha}^{2}}\right\rangle, \quad \mathcal{L}_{a b}^{(\alpha)}=\left\langle\frac{J_{0 \alpha}^{2} \Omega_{\mathrm{d} \alpha}^{a} \Omega_{\mathrm{tr} \alpha}^{b}}{1-\Omega_{\mathrm{tr} \alpha}^{2}}\right\rangle, \quad \mathcal{L}_{a b c}^{(\alpha)}=\left\langle\frac{J_{0 \alpha}^{2} \Omega_{\mathrm{d} \alpha}^{a} \Omega_{\mathrm{tr} \alpha}^{b} \Omega_{* \alpha}^{c}}{1-\Omega_{\mathrm{tr} \alpha}^{2}}\right\rangle
$$

onde, para seu cálculo, é conveniente observar que

$$
\frac{1}{1-\Omega_{\mathrm{tr} \alpha}^{2} / \omega^{2}}=\frac{\zeta_{\alpha}}{2}\left(\frac{1}{v_{\|} / v_{T_{\alpha}}+\zeta_{\alpha}}-\frac{1}{v_{\|} / v_{T_{\alpha}}-\zeta_{\alpha}}\right), \quad \zeta_{\alpha}=\frac{\omega}{\omega_{\mathrm{tr} \alpha}},
$$

pois esta observação nos permite obter a seguinte relação:

$$
\frac{1}{\sqrt{\pi}} \int_{-\infty}^{\infty} \frac{d v_{\|}}{v_{T_{\alpha}}} \frac{\left(v_{\|} / v_{T_{\alpha}}\right)^{n} \mathrm{e}^{-v_{\|}^{2} / v_{T_{\alpha}}^{2}}}{1-\Omega_{\operatorname{tr} \alpha}^{2}}=\frac{\zeta_{\alpha}}{2} D_{n}\left(\zeta_{\alpha}\right) .
$$

Segue, portanto, que

$$
\begin{aligned}
& \mathcal{L}_{0}^{(\alpha)}=\frac{\zeta_{\alpha}}{2} D_{0}\left(\zeta_{\alpha}\right)\left(1-\frac{1}{2} \mathrm{k}_{r}^{2} \rho_{\alpha}^{2}\right), \quad \mathcal{L}_{1}^{(\alpha)}=\frac{\omega_{\mathrm{d} \alpha}}{\omega} \frac{\zeta_{\alpha}}{2}\left[\frac{1}{2} D_{0}\left(\zeta_{\alpha}\right)+D_{2}\left(\zeta_{\alpha}\right)\right], \\
& \mathcal{L}_{2}^{(\alpha)}=\frac{\omega_{\mathrm{d} \alpha}^{2}}{\omega^{2}} \frac{\zeta_{\alpha}}{2}\left[\frac{1}{2} D_{0}\left(\zeta_{\alpha}\right)+D_{2}\left(\zeta_{\alpha}\right)+D_{4}\left(\zeta_{\alpha}\right)\right],
\end{aligned}
$$




$$
\begin{gathered}
\mathcal{L}_{01}^{(\alpha)}=\frac{1}{2} D_{1}\left(\zeta_{\alpha}\right), \\
\mathcal{L}_{02}^{(\alpha)}=\frac{1}{2 \zeta_{\alpha}} D_{2}\left(\zeta_{\alpha}\right), \\
\mathcal{L}_{11}^{(\alpha)}=\frac{\omega_{\mathrm{d} \alpha}}{\omega} \frac{1}{2}\left[\frac{1}{2} D_{1}\left(\zeta_{\alpha}\right)+D_{3}\left(\zeta_{\alpha}\right)\right] \\
\mathcal{L}_{001}^{(\alpha)}=\frac{\omega_{* \alpha}}{\omega} \frac{\zeta_{\alpha}}{2}\left[\left(1-\frac{\eta_{\alpha}}{2}\right) D_{0}\left(\zeta_{\alpha}\right)+\eta_{\alpha} D_{2}\left(\zeta_{\alpha}\right)\right] \\
\mathcal{L}_{101}^{(\alpha)}=\frac{\omega_{\mathrm{d} \alpha} \omega_{* \alpha}}{\omega^{2}} \frac{\zeta_{\alpha}}{2}\left[\left(\frac{1}{2}+\frac{\eta_{\alpha}}{4}\right) D_{0}\left(\zeta_{\alpha}\right)+D_{2}\left(\zeta_{\alpha}\right)+\eta_{\alpha} D_{4}\left(\zeta_{\alpha}\right)\right] \\
\mathcal{L}_{011}^{(\alpha)}=\frac{\omega_{* \alpha}}{\omega} \frac{1}{2}\left[\left(1-\frac{\eta_{\alpha}}{2}\right) D_{1}\left(\zeta_{\alpha}\right)+\eta_{\alpha} D_{3}\left(\zeta_{\alpha}\right)\right] \\
\mathcal{L}_{111}^{(\alpha)}=\frac{\omega_{\mathrm{d} \alpha} \omega_{* \alpha}}{\omega^{2}} \frac{1}{2}\left[\left(\frac{1}{2}+\frac{\eta_{\alpha}}{4}\right) D_{1}\left(\zeta_{\alpha}\right)+D_{3}\left(\zeta_{\alpha}\right)+\eta_{\alpha} D_{5}\left(\zeta_{\alpha}\right)\right]
\end{gathered}
$$

e, finalmente, com a substituição dos valores mostrados em (F.29), resulta:

$$
\begin{aligned}
& \mathcal{L}_{0}^{(\alpha)}=-\zeta_{\alpha}\left[Z\left(\zeta_{\alpha}\right)-i \sqrt{\pi} \mathrm{e}^{-\zeta_{\alpha}^{2}}\right]\left(1-\frac{\mathrm{k}_{r}^{2} \rho_{\alpha}^{2}}{2}\right), \\
& \mathcal{L}_{1}^{(\alpha)}=-\left\{\zeta_{\alpha}^{2}+\left(\frac{1}{2} \zeta_{\alpha}+\zeta_{\alpha}^{3}\right)\left[Z\left(\zeta_{\alpha}\right)-i \sqrt{\pi} \mathrm{e}^{-\zeta_{\alpha}^{2}}\right]\right\} \frac{\mathrm{k}_{r} \rho_{\alpha}}{\Omega}, \\
& \mathcal{L}_{2}^{(\alpha)}=-\left\{\frac{3}{2} \zeta_{\alpha}^{2}+\zeta_{\alpha}^{4}+\left(\frac{1}{2} \zeta_{\alpha}+\zeta_{\alpha}^{3}+\zeta_{\alpha}^{5}\right)\left[Z\left(\zeta_{\alpha}\right)-i \sqrt{\pi} \mathrm{e}^{-\zeta_{\alpha}^{2}}\right]\right\} \frac{\mathrm{k}_{r}^{2} \rho_{\alpha}^{2}}{\Omega^{2}}, \quad \mathcal{L}_{01}^{(\alpha)}=-\zeta_{\alpha} i \sqrt{\pi} \mathrm{e}^{-\zeta_{\alpha}^{2}}, \\
& \mathcal{L}_{02}^{(\alpha)}=-\left[1+\zeta_{\alpha} Z\left(\zeta_{\alpha}\right)\right]\left(1-\frac{\mathrm{k}_{r}^{2} \rho_{\alpha}^{2}}{2}\right), \quad \mathcal{L}_{11}^{(\alpha)}=-\left(\frac{1}{2} \zeta_{\alpha}+\zeta_{\alpha}^{3}\right) \frac{\mathrm{k}_{r} \rho_{\alpha}}{\Omega} \sqrt{\pi} \mathrm{e}^{-\zeta_{\alpha}^{2}}, \\
& \mathcal{L}_{001}^{(\alpha)}=-\left\{\eta_{\alpha} \zeta_{\alpha}^{2}+\left[\left(1-\frac{1}{2} \eta_{\alpha}\right) \zeta_{\alpha}+\eta_{\alpha} \zeta_{\alpha}^{3}\right]\left[Z\left(\zeta_{\alpha}\right)-i \sqrt{\pi} \mathrm{e}^{-\zeta_{\alpha}^{2}}\right]\right\} \frac{\Omega_{* \alpha}}{\Omega}, \\
& \mathcal{L}_{101}^{(\alpha)}=-\left\{\left(1+\frac{1}{2} \eta_{\alpha}\right) \zeta_{\alpha}^{2}+\eta_{\alpha} \zeta_{\alpha}^{4}+\left[\left(\frac{1}{2}+\frac{1}{4} \eta_{\alpha}\right) \zeta_{\alpha}+\zeta_{\alpha}^{3}+\eta_{\alpha} \zeta_{\alpha}^{5}\right]\left[Z\left(\zeta_{\alpha}\right)-i \sqrt{\pi} \mathrm{e}^{-\zeta_{\alpha}^{2}}\right]\right\} \frac{\Omega_{* \alpha} \mathrm{k}_{r} \rho_{\alpha}}{\Omega^{2}}, \\
& \mathcal{L}_{011}^{(\alpha)}=-\left[\left(\frac{1}{2}-\frac{1}{2} \eta_{\alpha}\right) \zeta_{\alpha}+\eta_{\alpha} \zeta_{\alpha}^{3}\right] \frac{\Omega_{* \alpha}}{\Omega} i \sqrt{\pi} \mathrm{e}^{-\zeta_{\alpha}^{2}} \\
& \mathcal{L}_{111}^{(\alpha)}=-\left[\left(\frac{1}{2}+\frac{1}{4} \eta_{\alpha}\right) \zeta_{\alpha}+\zeta_{\alpha}^{3}+\eta_{\alpha} \zeta_{\alpha}^{5}\right] \frac{\Omega_{* \alpha} \mathrm{k}_{r} \rho_{\alpha}}{\Omega^{2}} i \sqrt{\pi} \mathrm{e}^{-\zeta_{\alpha}^{2}} .
\end{aligned}
$$




\section{F.5 Obtenção do limite de fluido a partir das integrais com efeitos cinéticos}

No limite $\left|\zeta_{i}\right| \rightarrow \infty$, o termo $\mathrm{e}^{-\zeta_{i}^{2}}$ pode ser desprezado e, ao utilizar o limite assimptótico de $Z\left(\zeta_{i}\right)$, mostrado em (F.24), obtemos a partir de (F.36) o limite de fluido:

$$
\begin{aligned}
& \mathcal{L}_{0}^{(i)} \approx I_{0}^{(i)}+I_{02}^{(i)} \approx\left(1-\frac{1}{2} \mathrm{k}_{r}^{2} \rho_{i}^{2}\right)\left(1+\frac{1}{2 q^{2} \Omega^{2}}\right), \quad \mathcal{L}_{1}^{(i)}=I_{1}^{(i)}+I_{12}^{(i)}=\left(1+\frac{1}{q^{2} \Omega^{2}}\right) \frac{\mathrm{k}_{r} \rho_{i}}{\Omega}, \\
& \mathcal{L}_{2}^{(i)}=I_{2}^{(i)}+I_{22}^{(i)}=\left(\frac{7}{4}+\frac{23}{8} \frac{1}{q^{2} \Omega^{2}}\right) \frac{\mathrm{k}_{r}^{2} \rho_{i}^{2}}{\Omega^{2}}, \quad \mathcal{L}_{11}^{(i)}=I_{11}^{(i)}=0, \\
& \mathcal{L}_{101}^{(i)}=I_{101}^{(i)}+I_{121}^{(i)}=\left[1+\eta_{i}+\frac{1+2 \eta_{i}}{q^{2} \Omega^{2}}\right] \frac{\Omega_{* i} \mathrm{k}_{r} \rho_{i}}{\Omega^{2}}, \quad \mathcal{L}_{111}^{(i)}=I_{111}^{(i)}=0, \quad \mathcal{L}_{011}^{(i)}=I_{011}^{(i)}=0, \\
& \mathcal{L}_{001}^{(i)}=I_{001}^{(i)}+I_{021}^{(i)}=\left[1+\frac{1+\eta_{i}}{2 q^{2} \Omega^{2}}-\frac{1}{2}\left(1+\eta_{i}+\frac{1+2 \eta_{i}}{2 q^{2} \Omega^{2}}\right) \mathrm{k}_{r}^{2} \rho_{i}^{2}\right] \frac{\Omega_{* i}}{\Omega},
\end{aligned}
$$




\section{Apêndice G}

\section{Participação em eventos científicos}

\section{G.1 Cursos internacionais:}

- 8th Carolus Magnus Summer School on Plasma Physics and Fusion Science [103]: Este curso de curta duração, que ocorreu de 3 a 14 de setembro de 2007 em Bad Honnef na Alemanha, consistiu de inúmeros seminários sobre os principais tópicos a respeito de Física de Plasma e Fusão Nuclear (Magnetohidrodinâmica, Teoria cinética, Aquecimento, Transporte, etc...) com ênfase em aplicações para o ITER. Os seminários, que foram ministrados por pesquisadores e professores especialistas em cada área, foram publicados no formato de artigo breve em [2].

\section{G.2 Produção bibliográfica}

- Drif effects on geodesic acoust modes: Foi aceito em 2012 e publicado na versão final em 2013 [77]. Este trabalho foi, em grande parte, resultado da colaboração com Prof. Dr. A. I. Smolyakov pertencente a Universidade de Saskatchewan e é a base para propostas de trabalhos futuros que pretendemos realizar.

- Rotation effect on geodesic and zonal flow modes in tokamak plasmas with isothermal magnetic surfaces: O tema deste trabalho foi o alvo da pesquisa do mes- 
trado precedente ao presente doutorado [41] e do início deste doutorado. Na forma final, considerando fluxo de calor e equilíbrio com superfícies magnéticas isotérmicas, este trabalho foi publicado em 2011 [76].

\section{G.3 Conferências e encontros científicos}

- 40th European Physical Society Conference on Plasma Physics [104]: Trata-se de uma conferência em física de plasmas que ocorreu de 1 a 5 de julho de 2013 na Finlândia. Apresentamos nesta conferência um poster intitulado como "Diamagnetic effects and Landau Damping on geodesic acoustic modes" e um artigo no tamanho máximo permitido (4 páginas) [89]. Este trabalho é a base para o captítulo 4 desta tese e, a partir dele, pretendemos, no futuro, submeter um artigo com maior detalhamento algébrico em alguma revista internacional adequada ao tipo de trabalho.

- $12^{\circ}$ Encontro Brasileiro de Física de Plasmas [105]: Foi um evento científico nacional que ocorreu de 1 a 5 de Dezembro de 2013 na Universidade de Brasília (UnB) em Brasília (DF). Neste evento apresentamos, na forma de poster, a metodologia para um estudo sobre automodos acústicos geodésicos, cuja aplicação de maior impacto imediato está na determinação do perfil radial da temperatura de íons em tokamaks por meio de um novo tipo de diagnóstico em desenvolvimento: Espectroscopia com GAM. Trata-se de um tema que pretendemos desenvolver melhor em um trabalho futuro, no entanto, os conceitos iniciais e a metodologia para o desenvolvimento deste trabalho foram apresentados no poster e no resumo intitulados "Geodesic acoustic eigenmodes in the presence of diamagnetic effects" (P023) [106]. 


\section{Referências Bibliográficas}

[1] http://www.iter.org/ (Acessado em Julho de 2014).

[2] $8^{\text {th }}$ Carolus Magnus Summer School on Plasma and Fusion Energy Physics, Fusion Sci. Techn., 53, 2T (2008).

[3] I. Tamm and A. Sakharov, Theory of magnetic thermonuclear reactor, in Plasma physics and the problem of controlled thermonuclear reactions, 1, 3 (1959). Edited by M. A. Leontovich, published by Pergamon Press, London \& New York.

[4] J. Wesson, Tokamaks (2004), Clarendon Press Oxford.

[5] J. P. Freidberg, Plasma physics and fusion energy (2007), Cambridge University Press, New York.

[6] F. F. Chen, An indispensable truth: How fusion power can save the planet (2011), Springer.

[7] R. B. White, Theory of tokamak plasma (1989), North-Holland, Amsterdam.

[8] H. Alfvén, Existence of electromagnetic-hydrodynamics waves, Nature, 150, 405 (1942).

[9] A. G. Elfimov, A. S. de Assis, C. A. de Azevedo, N. I. Grishanov, F. M. Nekrasov, I. F. Potapenko, and V. S. Tsypin, Alfvén wave heating and current drive analysis in magnetized plasma structures, Braz. J. Phys., 25, 224 (1995). 
[10] F. Wagner and et. al., Regime of improved confinement and high beta in neutralbeam-heated discharges of the ASDEX tokamak divertor, Phys. Rev. Lett., 49, $1408(1982)$.

[11] H. Diamond, S. I. Itoh, K. Itoh, and T. S. Hahm, Zonal flows in plasma - a review, Plasma Phys. Control. Fusion, . 47, R35 (2005).

[12] M. N. Rosenbluth and F. L. Hinton, Poloidal flow driven by Ion-TemperatureGradient turbulence in tokamaks, Phys. Rev. Lett., 80, 724 (1998).

[13] K. Itoh, K. Hallatschek, and S. I. Itoh, Excitation of geodesic acoustic mode in toroidal plasmas, Plasma Phys. Control. Fusion, 47, 451 (2005).

[14] A. D. Turnbull, E. J. Strait, W. W. Heidbrink, M. S. Chu, H. H. Duong, J. M. Greene, L. L. Lao, T. S. Taylor, and S. J. Thompson, Global Alfvén modes: Theory and experiment, Phys. Fluids B, 6, 2546 (1993).

[15] W. W. Heidbrink, E. J. Strait, M. S. Chu, and A. D. Turnbull, Observation of BetaInduced Alfvén eigenmodes in the DIII-D tokamak, Phys. Rev. Lett., 71, 855 (1993).

[16] W. W. Heidbrink, E. Ruskov, E. M. Carolipio, J. Fang, M. A. van Zeeland, and R. A. James, What is the "Beta-Induced Alfvén Eigenmode" ?, Phys. Plasmas, 6, 1147 (1999).

[17] W. W. Heidbrink, Basic physics of Alfvén instabilities driven by energetic particles in toroidally confined plasmas, Phys. Plasmas, 15, 055501 (2008).

[18] F. Zonca, L. Chen, and R. Santoro, Kinetic theory of low-frequency Alfvén modes in tokamaks, Plasma Phys. Control. Fusion, 38, 2011 (1996).

[19] A. Fasoli, D. Testa, S. Sharapov, H. L. Berk, B. Breizman, A. Gondhalekar, R. F. Heeter, M. Mantsinen, and W. E. J., MHD spectroscopy, Plasma Phys. Control. Fusion, 44, B159 (2002). 
[20] S. E. Sharapov, D. Testa, B. Alper, D. N. Borba, A. Fasoli, N. C. Hawkes, R. F. Heeter, M. Mantsinen, and M. G. Von Hellermann, MHD spectroscopy through detecting toroidal Alfvén eigenmodes and Alfvén wave cascades, Phys. Lett. A, 289, 127 (2001).

[21] A. G. Elfimov, Kinetic ion effect on geodesic acoustic alfven modes in tokamaks, Phys. Plasmas, 16, 034501 (2009).

[22] G. D. Conway, C. Troster, B. Scott, and K. Hallatschek, Frequency scaling and localization of geodesic acoustic modes in ASDEX Upgrade, Plasma Phys. Control. Fusion, 50, 055009 (2008).

[23] A. Fujisawa, A review of zonal flow experiments, Nuclear Fusion, 49, 013001 (2009).

[24] G. R. McKee, D. K. Gupta, R. J. Fonck, D. J. Schlossberg, M. W. Shafer, and Gohil, Structure and scaling properties of the geodesic acoustic mode, Plasma Phys. Control. Fusion, 48, S123 (2006).

[25] A. V. Melnikov, V. A. Vershkov, L. G. Eliseev, S. A. Grashin, A. V. Gudozhnik, L. I. Krupnik, S. E. Lysenko, V. A. Mavrin, S. V. Perfilov, D. A. Shelukhin, S. V. Soldatov, M. V. Ufimtsev, A. O. Urazbaev, G. Van Oost, and L. G. Zimeleva, Investigation of geodesic acoustic mode oscillations in the T-10 tokamak, Plasma Phys. Control. Fusion, 48, S87 (2006).

[26] N. Winsor, J. L. Johnson, and J. M. Dawson, Geodesic acoustic waves in hydromagnetic systems, Phys. Fluids, 11, 2448 (1968).

[27] V. P. Lakhin, V. I. Ilgisonis, and A. I. Smolyakov, Geodesic acoustic modes and zonal flows in toroidally rotating tokamak plasmas, Phys. Lett. A, 374, 4872 (2010).

[28] S. I. Braginskii, Transport processes in a plasma, in Reviews of Plasma Physics, 1, 205, Edited by M. A. Leontovich, published by Consultants Bureau, New York (1965). 
[29] V. B. Lebedev, N. Yushmanov, H. Diamond, S. V. Novakovskii, and A. I. Smolyakov, Plateau regime dynamics of the relaxation of poloidal rotation in tokamak plasmas, Phys. Plasmas, 3, 3023 (1996).

[30] R. D. Hazeltine and J. D. Meiss, Plasma Confinement (1992), Addison-Wesley Publishing Company.

[31] J. A. Bittencourt, Fundamentals of Plasma Physics. New York, Pergamon Press (1995).

[32] J. Catto, W. M. Tang, and D. E. Baldwin, Generalized gyrokinetics, Plasma Phys., 23, 639 (1981).

[33] L. D. Landau, On the vibration of the electronic plasma,J. Phys. USSR, 10, 25 (1946).

[34] A. B. Mikhailovskii, "Drift" instabilities distorting the magnetic surfaces of Tokamak-type toroidal systems, Nucl. Fusion, 13, 259 (1973).

[35] A. G. Elfimov, Alfvén continuum deformation by kinetic geodesic effect in rotating tokamak plasmas, Phys. Plasmas, 17, 022102 (2010).

[36] A. I. Smolyakov, X. Garbet, G. Falchetto, and M. Ottaviani, Multiple polarization of geodesic curvature induced modes, Physics Letters A, 372, 6750 (2008).

[37] A. I. Smolyakov, Gyroviscous forces in a collisionless plasma with temperature gradients, Canadian J. Phys., 76, 321 (1998).

[38] F. F. Chen, Introduction to plasma physics and controlled fusion (1984), Springer, 2nd ed..

[39] H. Goedbloed and S. Poedts, Principles of Magnetohydrodynamics, 1 (2004), Cambridge.

[40] R. Balescu, Aspect of anomalous transport (2005), North-Holland, Amsterdam. 
[41] R. J. F. Sgalla, Efeito de rotação nos fluxos zonais e modos acústicos geodésicos, Master's thesis (2010), Institute of Physics - University of São Paulo (IFUSP), Brazil.

[42] B. A. Trubnikov, Particle interactions in a fully ionized plasma, in Reviews of Plasma Physics, 1, 105 (1965), Edited by M. A. Leontovich, published by Consultants Bureau, New York.

[43] D. V. Sivukhin, Coulomb collisions in a fully ionized plasma, in Reviews of Plasma Physics, 4, 93 (1966), Ed. by M. A. Leontovich, published by Consultants Bureau, New York.

[44] Helander and D. J. Sigmar, Collisional Transport in Magnetized Plasmas (2002), Cambridge Monographs on Plasma Physics.

[45] R. J. Goldston and H. Rutherford, Introduction to plasma physics (1995), IOP Publishing Ltd.

[46] J. P. Freidberg, Ideal Magnetohydrodynamics (1987), Plenum Press, New York \& London.

[47] H. Grad and H. Rubin, Hydromagnetic equlibria and force-free fields, in Proceedings of the 2nd UN Conf. on the Peaceful Uses of Atomic Energy (IAEA, Geneva), 31, 190 (1958).

[48] V. D. Shafranov, Plasma equilibrium in a magnetic field, in Reviews of Plasma Physics, 2, 103 (1966), Ed. by M. A. Leontovich, published by Consultants Bureau, New York.

[49] E. K. Maschke and H. Perrin, Exact solutions of the stationary MHD equations for a rotating toroidal plasma, Plasma Phys., 22, 579 (1980)

[50] V. I. Ilgisonis and et al., Geodesic acoustic modes and zonal flows in rotating large-aspect-ratio tokamak plasmas, Plasma Phys. Control. Fusion, 53, 065008 (2011). 
[51] V. I. Ilgisonis and I. Pozdnyakov Yu, Transport barrier as a bifurcation of the equilibrium of a tokamak plasma, Plasma Phys. Rep., 28, 83 (2002).

[52] M. Kruskal and J. L. Tuck, The Instability of a pinched fluid with a longitudinal magnetic field, Proc. R. Soc. (London), Ser. A 245, 222 (1958).

[53] V. Shafranov, On the stability of a cylindrical gaseous conductor in a magnetic field, J. NUclear Energy, 5, 86 (1957).

[54] R. Balescu, Transport processes in a plasma, 1 (1988), North-Holland, Amsterdam.

[55] R. Balescu, Transport processes in a plasma, 2 (1988), North-Holland, Amsterdam.

[56] F. L. Hinton and R. D. Hazeltine, Theory of plasma transport in toroidal confinement systems, Rev. Mod. Phys., 48, 239 (1976).

[57] S. P. Hirshman and D. J. Sigmar, Neoclassical transport of a multispecies toroidal plasma in various collisionality regimes, Phys. Fluids, 20, 418 (1977).

[58] A. A. Galeev and R. Z. Sagdeev, Theory of neoclassical diffusion, in Reviews of Plasma Physics, 7, 257 (1979), Edited by M. A. Leontovich, publisehd by Consultants Bureau, New York.

[59] S. P. Hirshman and D. J. Sigmar, Neoclassical transport of impurities in tokamak plasmas, Nucl. Fusion, 21, 1079 (1981).

[60] D. Bohm, The characteristics of electrical discharges in magnetic fields (1949), Edited by A. Guthrie and R. K. Wakerling, published bu McGraw-Hill, New York.

[61] A. I. Morozov and L. S. Soloviev, Motion of charged particles in electromagnetic fields, 2, 201 (1966), Edited by M. A. Leontovich, published by Consultants Bureau, New York.

[62] http://fap.if.usp.br/〜renee/NOTAS/gyro_segundo.pdf (Acessado em Julho de 2014). 
[63] R. D. Hazeltine and F. Waelbroeck, The Framework of Plasma Physics (2004). Westview, Boulder CO.

[64] A. I. Smolyakov, C. Nguyen, and X. Garbet, Kinetic theory of electromagnetic geodesic acoustic modes, Plasma Phys. Control. Fusion, 50, 115008 (2008).

[65] A. B. Mikhailovskii and S. E. Sharapov, Beta-induced temperature-gradient eigenmodes in tokamaks: II. Kinetic theory, Plasma Phys. Reports, 25, 838 (1999).

[66] F. Zonca and L. Chen, Radial structures and nonlinear excitation of geodesic acoustic modes, Europhys. Lett., 83, 35001 (2008).

[67] G. Y. Fu and C. Z. Cheng, Theory of a high-n toroidicity-induced shear Alfvén eigenmode in tokamaks, Phys. Fluids B, 2, 985 (1990).

[68] D. Zhou, Electromagnetic geodesic acoustic modes in tokamak plasmas, Phys. Plasmas, 14, 104502 (2007).

[69] R. Hazeltine, Recursive derivation of drift-kinetic equation, Plasma Phys., 15, 77 (1973).

[70] H. Grad, On the kinetic theory of rarefied gases, Communications on Pure and Applied Mathematics, 2, 331 (1949).

[71] H. Grad, Toroidal Containment of a Plasma, Phys. Fluids, 10, 137 (1967).

[72] J. P. Wang and J. D. Callen, Fluid/kinetic hybrid moment description of plasmas via a Chapman-Enskog-like approach, Phys. Fluids B, 4, 1139 (1992).

[73] A. B. Mikhailovskii and V. S. Tsypin, Transport equations of plasma in a curvilinear magnetic field, Beitrage Plasma Physik, 24, 335 (1984).

[74] C. T. Hsu, R. D. Hazeltine, and J. Morrison, A generalized reduced fluid model with finite ion-gyroradius effects, Physics of Fluids, 29, 1480 (1986). 
[75] A. I. Smolyakov, X. Garbet, and C. Bourdelle, On the parallel momentum balance in low pressure plasmas with an inhomogeneous magnetic field, Nucl. Fusion, 49, 125001 (2009).

[76] A. G. Elfimov, R. M. O. Galvao, and R. J. F. Sgalla, Rotation effect on geodesic and zonal flow modes in tokamak plasmas with isothermal magnetic surfaces, Plasma Phys. Control. Fusion, 53, 105003 (2011).

[77] R. J. F. Sgalla, A. I. Smolyakov, A. Elfimov, and M. F. Bashir, Drift effects on geodesic acoustic modes, Phys. Lett. A, 377, 303 (2013).

[78] R. M. O. Galvão, W. A. H. Baquero, I. L. Caldas, N. A. M. Cuevas, V. N. Duarte, A. G. Elfimov, J. I. Elizondo, A. M. M. Fonseca, T. M. Germano, Z. O. Guimarães-Filho, J. L. Jeronimo, Yu. K. Kuznetsov, M. A. M. Manrique, I. C. Nascimento, C. J. A. Pires, P. G. P. Puglia, A. P. Reis, G. Ronchi, L. F. Ruchko, W. P. De Sá, R. J. F. Sgalla, E. K. Sanada, J. H. F. Severo, V. C. Theodoro, C. H. S. Amador, F. Borges, D. L. Toufen, Report on recent results obtained in TCABR, 15 th Latin American Workshop on Plasma Physics, R-PT1 (2014).

[79] J. H. F. Severo, I. C. Nascimento, V. S. Tsypin and R. M. O. Galvão, Plasma residual rotation in the TCABR tokamak, Nucl. Fusion, 43, 1047 (2003)

[80] H. L. Berk, C. J. Boswell, D. Borba, A. C. A. Figueiredo, T. Johnson, M. F. F. Nave, S. D. Pinches, S. E. Sharapov and JET EFDA contributors, Explanation of the JET $\mathbf{n}$ $=\mathbf{0}$ chirping mode, Nucl. Fusion, 46, S888 (2006)

[81] A. Krämer-Flecken, S. Soldatov, K. H. R., O. Zimmermann, and T. Team, Acoustic modes and the relation to density fluctuations, Phys. Rev. Lett., 97, 045006 (2006).

[82] A. I. Smolyakov, C. Nguyen, and X. Garbet, Electromagnetic effects on geodesic acoustic and beta-induced alfvén eigenmodes, Nucl. Fusion, 50, 054002 (2010). 
[83] G. N. Throumoulopoulos, H. Weitzner, and H. Tasso, On nonexistence of tokamak equilibria with purely poloidal flow, Phys. Plasmas, 13, 122501 (2006).

[84] B. D. Fried and S. D. Conte, The Plasma Dispersion Function (1961), Academic Press, New York.

[85] H. Sugama and T. H. Watanabe, Collisionless damping of geodesic acoustic modes, J. Plasma Phys., 72, 825 (2006).

[86] P. communication with A. Hirose (2012)

[87] Yu. K. Kuznetsov, I. C. Nascimento1, C. Silva, H. Figueiredo, Z.O. Guimarães-Filho, I. L. Caldas, R. M. O. Galvão, J. H. F. Severo, D. L. Toufen, L. F. Ruchko, A. G. Elfimov, J. I. Elizondo, W. P. de Sá, O.C. Usuriaga, E. Sanada, A. V. Melnikov, M. P. Gryaznevich, M. Peres Alonso, A. P. Reis, M. Machida, D. J. Trembach, T. M. Germano, R. Narayanan, M. Ghoranneviss, R. Arvin, S. Mohammadi, S. R. S. Tekieh, F.O. Borges, V. Bellintani, G.P. Canal, P. Duarte, R. M. de Castro, G. Vorobyov, M. Mizintseva, V. E. Moiseenko, F. do Nascimento, G. Ronchi and L. M. F. Schmutzler, Long-distance correlations in TCABR biasing experiments, Nucl. Fusion, 52, 063004 (2012)

[88] W. Horton, Drift waves and transport, Rev. Mod. Phys., 71, 735 (1999).

[89] R. J. F. Sgalla, A. G. Elfimov, and A. I. Smolyakov, Diamagnetic effects and Landau damping on geodesic acoustic modes, 40th EPS Conference on Plasma Physics, P2.183 (2013).

[90] F. Zonca, L. Chen, A. Botrugno, Buratti, A. Cardinali, R. Cesario, V. Pericoli Ridolfini and JET-EFDA contributors, High-frequency fishbones at JET: theoretical interpretation of experimental observations, Nucl. Fusion, 49, 085509 (2009).

[91] Lauber and S. Gunter, Damping and drive of low-frequency modes in tokamak plasmas, Nucl. Fusion, 48, 084002 (2008). 
[92] Lauber, M. Bruedgam, D. Curran, V. Igochine, K. Sassenberg, S. Guenter, M. Maraschek, M. Garcia-Munoz, N. Hicks, and ASDEX Upgrade Team, Kinetic Alfvén eigenmodes at ASDEX Upgrade, Plasma Phys. Control. Fusion, 51, 124009 (2009)

[93] I. Chavdarovski and F. Zonca, Effects of trapped particle dynamics on the structures of a low-frequency shear alfvén continuous spectrum, Plasma Phys. Control. Fusion, 51, 115001 (2009).

[94] F. M. Nekrasov, A. G. Elfimov, V. S. Tsypin, C. A. de Azevedo, and A. S. de Assis, The parallel permitivity of magnetized toroidal plasmas with elliptic magnetic surfaces, Czechoslovak J. Phys., 46, 565 (1996).

[95] E. S. Cheb-Terrab and A. G. Elfimov, The permittivity tensor and bounce resonance effects on wave dissipation in toroidal plasmas, Czechoslovak J. Phys., 46, 595 (1996).

[96] F. M. Nekrasov, A. G. Elfimov, C. A. de Azevedo, and A. S. de Assisb, The coulomb scattering effect on trapped particles bounce-resonance dissipation in magnetized toroidal plasmas, Phys. Plasmas, 6, 1547 (1999).

[97] D. Zhou, Zonal flow modes in a tokamak plasma with dominantly poloidal mean flows, Phys. Plasmas, 17, 102505 (2010).

[98] K. Itoh, S.-I. Itoh, H. Diamond, A. Fujisawa, M. Yagi, T. Watari, Y. Nagashima, and A. Fukuyama, Geodesic acoustic eigenmodes, Plasma and Fusion Research: Rapid Communication, 1, $037(2006)$

[99] S.-I. Itoh, K. Itoh, M. Sasaki, A. Fujisawa, T. Ido, and Y. Nagashima, Geodesic acoustic mode spectroscopy, Plasma Phys. Control. Fusion, 49, L7 (2007)

[100] I. O. Pogutse, A. I. Smolyakov, and A. Hirose, Magnetohydrodynamic equations for plasmas with finite-Larmor-radius effects, J. Plasma Phys., 60, 133 (1998). 
[101] W. D. D' haeseleer, W. N. G. Hitchon, J. D. Callen, and J. L. Shohet, Flux coordinates and magnetic field structure: A guide to a fundamental tool of plasma theory (1991), Springer-Verlag Berlin Heidelberg.

[102] J. D. Huba, NRL Plasma formulary (2009), Supported by The Office of Naval Research Laboratory.

[103] http://www. carolusmagnus.net/ (Acessado em Julho de 2014).

[104] http://eps2013.aalto.fi/ (Acessado em Julho de 2014).

[105] http://www.sbfisica.org.br/ ebfp/xii/ (Acessado em Julho de 2014).

[106] http://www.sbf1.sbfisica.org.br/eventos/ebfp/12/programa/lista_trabalho. asp?sesId=11\&str=Sgalla (Acessado em Julho de 2014). 